\title{
Quantum features and advanced numerical modeling of strong-field ionization
}

\author{
PH.D. THESIS \\ written by \\ SZILÁRD MAJOROSI \\ University of Szeged, Department of Theoretical Physics
}

Supervisor:

AtTila CZIRJÁK

research fellow, honorary associate professor

University of Szeged, Department of Theoretical Physics

University of Szeged

Doctoral School of Physics

Faculty of Science and Informatics

Department of Theoretical Physics

Szeged, Hungary

2018 


\section{Contents}

\section{Introduction and motivation}

I Preliminaries 6

1 Quantum description of strong-field phenomena 7

1.1 Establishment of attosecond physics . . . . . . . . . . . . . . . 7

1.2 The time-dependent Schrödinger equation of an atomic system in an intense laser field . . . . . . . . . . . . . . . . . . . 8

1.2.1 The semiclassical description . . . . . . . . . . . 9

1.2.2 Three-dimensional model equation in cylindrical coordinates . . . . 10

1.2.3 One-dimensional modeling . . . . . . . . . . . . . 11

1.3 Strong-field ionization of the interacting electron - ion-core system . . . . . 13

1.4 Characterization of the strong-field ionization . . . . . . . . . . . . 14

1.4.1 Ionization types . . . . . . . . . . . . . . . . . . . . . 14

1.4 .2 Physical quantities . . . . . . . . . . . . . . . 16

2 Standard methods for the numerical solution of the TDSE 18

2.1 Crank-Nicolson method f . . . . . . . . . . . . . . . . 18

2.1.1 Approximation of the time evolution operator $\ldots \ldots \ldots$

2.1.2 The cylindrical finite difference scheme . . . . . . . . . . . . . 20

2.1.3 Approximating the cylindrical inner product . . . . . . . . . . . 22

2.2 Operator splitting schemes . . . . . . . . . . . . . . . . . . . . 24

2.2.1 Operator splitting formulas . . . . . . . . . . . . 24

2.2.2 Directional splitting of the exponential operator $\ldots \ldots . \ldots 26$

3 Theoretical description of entangled quantum systems 28

3.1 Quantification of bipartite entanglement . . . . . . . . . . . . . 28

3.1.1 Schmidt decomposition . . . . . . . . . . . . . . 28

3.1.2 Entanglement entropies . . . . . . . . . . . . . . . 29

3.2 Elements of quantum information theory $\ldots \ldots \ldots$. . . . . . . 30

3.2.1 Correlation types and quantum information . . . . . . . . . . 30

3.2.2 Quantum conditional entropy ................. 31 
3.2.3 Quantum mutual entropy . . . . . . . . . . . 32

II New results $\quad 34$

4 The hybrid splitting algorithm $\quad 35$

4.1 The Schrödinger equation and its boundary conditions . . . . . . . . 35

4.1.1 The boundary condition for the 3D singular Coulomb potential . . . 35

4.1.2 The states with nonzero magnetic quantum numbers . . . . . . . . . . 37

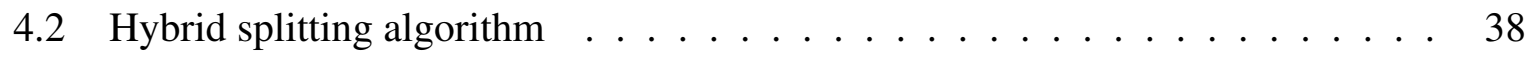

4.2.1 Hybrid splitting of the exponential operator . . . . . . . . . . . 38

4.2.2 Hybrid splitting solver algorithm . . . . . . . . . . . . 41

4.3 Results and discussion . . . . . . . . . . . . . . . . . . 45

4.3.1 Spatial accuracy: stationary state energies . . . . . . . . . . . . 45

4.3.2 Temporal accuracy: forced harmonic oscillator . . . . . . . . . . . . 47

4.3 .3 Hydrogen atom in an external field ............ 50

4.4 Summary . . . . . . . . . . . . . . . . . . . . 52

5 Quantum entanglement in strong-field ionization $\mathbf{5 4}$

5.1 Theoretical model . . . . . . . . . . . . . . . . . 54

5.2 Entanglement calculation . . . . . . . . . . . . . . . 55

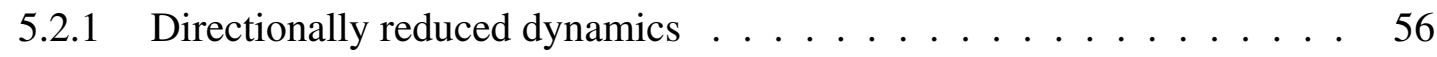

5.2 .2 Correlation quantification per direction . . . . . . . . . . 57

5.2 .3 Approximation of the entanglement . . . . . . . . . . . . . 59

5.3 Quantum entanglement in the one-dimensional model . . . . . . . . . . . 60

5.4 Results and discussion . . . . . . . . . . . . . . . . . . . . 62

5.4.1 Simulation parameters . . . . . . . . . . . . . 62

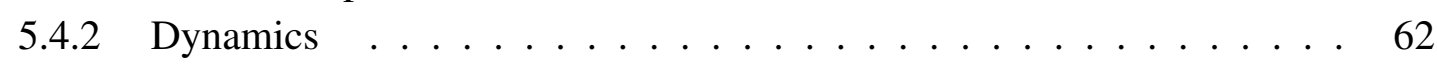

5.4.3 Time dependence of quantum entropies . . . . . . . . . . . . . 63

5.4.4 Dependence on the electric-field strength . . . . . . . . . . . . . . 67

5.4.5 Dependence on the carrier-envelope phase . . . . . . . . . . 69

5.5 Summary . . . . . . . . . . . . . . . . . . . . 72

6 One-dimensional density-based model potentials $\quad \mathbf{7 4}$

6.1 Theoretical model . . . . . . . . . . . . . . . . . . . . . . . 74

6.1.1 Derivation of the 1D analytical model potential . . . . . . . . . 75

6.1 .2 Improved 1D model potentials . . . . . . . . . . . . . . . . 76

6.2 Numerical model . . . . . . . . . . . . . . . . . . . . 77

6.2.1 Density-based numerical model potentials . . . . . . . . . . . 77

6.2.2 Test results: density-based 1D model potential . . . . . . . . . . . 79

6.2.3 Test results: 1D Dirac-delta potential _ . . . . . . . . . . . . 80

6.3 Results and comparison of the $1 \mathrm{D}$ and $3 \mathrm{D}$ calculations $\ldots \ldots \ldots$. . . . . 81

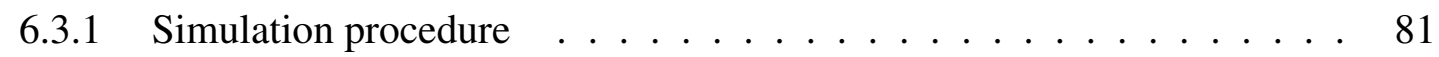

6.3.2 Low frequency response .................... 82 
6.3.3 High-order harmonic spectra . . . . . . . . . . . . . 85

6.3.4 Electron - ion-core quantum entanglement along direction z . . . . . . 87

6.4 Summary . . . . . . . . . . . . . . . . . . . 88

Summary of the thesis $\quad 90$

Összefoglalás $\quad 97$

$\begin{array}{ll}\text { Acknowledgements } & 105\end{array}$

$\begin{array}{ll}\text { Publications } & 106\end{array}$

$\begin{array}{ll}\text { Bibliography } & 107\end{array}$

$\begin{array}{ll}\text { Appendices } & 116\end{array}$

A High-order finite difference formulas 116

B The Numerov z-line propagator algorithm 118

$\begin{array}{lll}\text { C The forced harmonic oscillator } & 121\end{array}$ 


\section{Introduction and motivation}

Pioneering experiments with attosecond light pulses [1-6], based on high-order harmonic generation (HHG) in noble gases [7-10], have been revolutionizing our view of fundamental atomic, molecular and solid state processes in this time domain [11-16]. A key step in gas-HHG is the tunnel ionization of a single atom and the return of the just liberated electron to its parent ion due to the strong, linearly polarized femtosecond laser pulse driving this process [17-23]. Recent developments in attosecond physics revealed that the accurate description of this single-atom emission is more important than ever, e.g. for the correct interpretation of the data measured in attosecond metrology experiments [13, 24].

Although an intuitive and very successful approximate analytical solution [19] and many of its refinements exist [21], the most accurate description of the single-atom response is given by the numerical solution of the time-dependent Schrödinger equation (TDSE). The peculiarity of this problem is due to the electric field strength of the laser pulse, which has its maximum typically in the range of 0.05-0.1 atomic units, i.e. it enables the tunneling of the electron through the time-dependent potential barrier formed by strongly distorting the atomic potential, but this effect is weak during the whole process. On the other hand, the small liberated part of the wave function outside the barrier extends to large distances and in fact this is the main contribution to the time-dependent dipole moment, which is the source of the emitted radiation. Thus, a very weak effect needs to be computed very accurately, and these requirements get even more severe, if the model goes beyond the usually employed single-active-electron and dipole approximations. Regarding HHG, the fundamental importance of the singularity of the Coulomb potential has been analysed and emphasized e.g. in [22]. As other kind of numerical errors decrease, the artifacts caused by the smoothing of the singularity become more disturbing. Therefore, a high precision numerical method has to handle the Coulomb singularity as accurately and effectively as possible.

For linearly polarized pulses, the main dynamics happens along the electric field of the laser pulse which underlies the success of some one-dimensional (1D) approximations [2535]. These typically use various $1 \mathrm{D}$ model potentials to account for the behavior of the atomic system. However, the particular model potential chosen heavily influences the $1 \mathrm{D}$ results and their comparison with the true three-dimensional (3D) results is usually nontrivial. One of these important deviations is that the dipole moment, created by the same electric field, may have much larger or much smaller values in the $1 \mathrm{D}$ than in the 3D simulation. Therefore, a more elaborate connection between the $3 \mathrm{D}$ problem and its $1 \mathrm{D}$ model is necessary to allow the $1 \mathrm{D}$ simulation of strong-field processes physically as correctly as possible. 
Although strong-field ionization is widely used as a standard procedure to generate e.g. high-order harmonic radiation, it is very little known that this process generates also quantum entanglement between the liberated electron and its parent ion-core. Quantum entanglement is a fundamental feature of quantum theory which enables strong correlations, without classical counterpart, between the constituents of a quantum system. Despite the fact that the discussion of quantum entanglement between two particles' spatial motion dates back to the early days of quantum mechanics [36, 37], the features of continuous variable quantum entanglement [38] are still much less explored and utilized than those of discrete variables systems.

Most of the papers on quantum entanglement in light induced atomic processes study the correlations between the emitted photon and the emitting atomic system [39-41]. Papers on entanglement between a charged particle and a photon $[42,43]$, entanglement in two particles' collision [44-49] give also valuable insight into the quantum features of problems related to the present thesis. Entanglement between the fragments of an atomic system due to a lightinduced break-up process, like photoionization and photodissociation, was studied by Fedorov and coworkers $[50,51]$ in the framework of Gaussian states. However, this latter approach is not suitable to deal with the problem of quantum entanglement during the strong-field ionization of an atom, which motivated us to perform an accurate numerical investigation of the problem.

This thesis is organized as follows: Part I covers the theoretical preliminaries in three chapters. We give an overview of the quantum description of strong-field physics in Chapter 1 . In Chapter 2, we discuss standard numerical methods for the solution of the time-dependent Schrödinger equation that are relevant for this thesis. We devote Chapter 3 to review the standard quantification methods of correlations in bipartite quantum systems in pure or mixed states. Part II presents our new scientific results in three chapters. In Chapter 4, we introduce the elements and discuss the testing of our hybrid splitting algorithm that provides high-order accurate solution for the Schrödinger equation with Coulomb singularities in real space cylindrical coordinates. In Chapter 5, we present the results of our detailed investigations regarding the emergence of quantum entanglement during strong-field ionization. In Chapter 6, we advance the one-dimensional modeling of strong-field ionization by the development of density-based model potentials. We complete this thesis with summaries in English and in Hungarian. 


\section{Part I}

\section{Preliminaries}




\section{CHAPTER 1}

\section{Quantum description of strong-field phenomena}

In this chapter we provide a short introduction to the quantum description of strong-field phenomena. First, we briefly review the main developments of the past 30 years and highlight a few selected experimental results in this area, which are also relevant to some of the current problems of attosecond physics. Then we introduce the main model equation in this thesis, the time-dependent Schrödinger equation in cylindrical coordinates for a single-electron atom in an intense laser field of linear polarization. Next we refine this model equation of strongfield ionization by describing the atomic system as the interacting electron - ion-core two-body system. Finally, we summarize the several ionization types and regimes that might occur, and time-dependent physical quantities that can characterize the strong-field process.

\subsection{Establishment of attosecond physics}

By the end of the 1980s, the development of femtosecond laser technology, primarily the chirped pulse amplification, allowed to create laser pulses of such a high intensity that the electric field strength of the pulse was already comparable to that of the Coulomb field felt by an atomic electron. Strong-field physics experiments irradiating low density noble gas samples with these laser pulses discovered an unexpectedly intense high-order harmonic radiation [7-10] emitted by the gas. Within a few years, theoretical explanations (based partly on pioneering earlier works $[17,52]$ ) revealed the underlying physics of tunnel ionization $[18,19,53]$, and a very important idea emerged about the possibility of creating "light" pulses of attosecond duration, utilizing the characteristic spectrum of high-order harmonic radiation [9]. In less than a decade, attosecond XUV pulse-trains became reality in leading laser labs [2-6] which opened the possibility of experimenting with atomic, molecular and solid systems on truly atomic timescales [11-16]. Within a few additional years, generation of isolated attosecond pulses was also achieved [54, 55], allowing to measure processes well below 100 as.

Here we mention only a few of these ground-breaking experiments:

- The time needed for an atomic electron to tunnel through the barrier created by the laser electric field by distorting the atomic binding potential is of fundamental importance to strong-field and attosecond science. An experiment with Ne atoms used a 250 attosecond 
pulse, acting as the pump, to generate Ne ions in excited states, from which a timedelayed linearly polarized waveform-controlled few-cycle laser pulse, acting as the probe, liberates electrons to produce doubly charged Ne ions. The results revealed that tunneling takes place within less than 400 attosecond after excitation, dominantly around the peaks of the laser electric field [11].

- Another experiment with He atoms used elliptically polarized laser pulses to probe the time delay between the instant of strong-field ionization (dominantly by tunneling) and the peak of the laser pulse, measuring an upper limit of 34 attoseconds [16, 24]. A recent experiment [56] using a similar method suggests also several tens of attoseconds for the time spent under the barrier during tunnel ionization for $\mathrm{Kr}$ and $\mathrm{Ar}$ atoms.

- For more than a century, photoemission from atoms was considered to be an instantaneous response to suitable radiation. Measurements based on attosecond pulses revealed that the emission of electrons liberated from the $2 p$ orbitals of neon atoms have a delay of ca. 21 attoseconds compared to those from the 2 s orbital [13].

- As a first application of attosecond pulses to investigate a solid target, the time delay between photoelectrons originating from the core and from the valence band of a tungsten sample was measured to be ca. 110 attoseconds [12].

Most of the experiments in attosecond physics are pump-and-probe experiments, where typically light (in the broad sense, i.e. from infrared to XUV) and electrons or ions emitted by the target are detected as the function of the time-delay between the pump and the probe pulse. The typically near-infrared (NIR) few-cycle laser pulse, which created the isolated attosecond pulse, is frequently employed also in the measurement, in combination with the attosecond pulse, in order to utilize the "attosecond streaking": the laser pulse shifts the momentum of the liberated electron by the value proportional to its vector potential at the time instant when to electron was set (approximately) free. This allows (based on certain important assumptions) to retrieve this time instant from the measured data.

The experiments cited above are interesting not only for providing new information about the particular process, but they also help to improve theoretical models, which in turn refine the interpretation of data measured with attosecond metrology. Currently, fundamental open problems are e.g. the setting of the zero of time with sufficient precision, and the question about the momentum of the electron right after tunneling. For both of these problems, highly accurate numerical solution of the Schrödinger equation is extremely important.

\subsection{The time-dependent Schrödinger equation of an atomic system in an intense laser field}

In the following, we briefly overview the standard quantum theory of nonrelativistic laser-atom interaction. In Section 1.2.1 we introduce the time-dependent Schrödinger equation of a bound charged particle interacting with an electromagnetic field with suitable approximations. Next, 
in Section 1.2.1, we write out explicitly the form of the Schrödinger equation in cylindrical coordinates. We also discuss the simplified one-dimensional modeling of the problem in Section 1.2.3.

\subsubsection{The semiclassical description}

We describe the interaction between a charged particle and an electromagnetic field semiclassically: this means that we treat the external field as a classical quantity, but we use the nonrelativistic quantum mechanical description for the motion of the particle. Thus, we describe the Hamiltonian of the particle with charge $q$ and mass $m$ with the so-called minimal coupling [57]:

$$
\hat{H}^{\prime}(t)=\frac{1}{2 m}\left(\mathbf{P}-q \mathbf{A}^{\prime}(\mathbf{R}, t)\right)^{2}+V_{0}(\mathbf{R})-\frac{q}{m} \mathbf{B}(\mathbf{R}, t) \mathbf{S},
$$

where and $\mathbf{A}^{\prime}(\mathbf{R}, t)$ denotes the vector potential of the external electromagnetic field, $\mathbf{B}(\mathbf{R}, t)$ denotes its magnetic field, $\mathbf{S}$ denotes the spin angular momentum operator, and $V_{0}(\mathbf{R})$ a static scalar potential. The time-dependent Schrödinger equation then reads:

$$
i \hbar \frac{\partial}{\partial t}\left|\Psi^{\prime}(t)\right\rangle=\hat{H}^{\prime}(t)\left|\Psi^{\prime}(t)\right\rangle
$$

If we perform the gauge transformation

$$
\mathbf{A}(\mathbf{R}, t)=\mathbf{A}^{\prime}(\mathbf{R}, t)+\nabla_{\mathbf{R}} \chi(\mathbf{R}, t), \quad \text { and } \quad|\Psi(t)\rangle=\left|\Psi^{\prime}(t)\right\rangle e^{-i \frac{q}{\hbar} \chi(\mathbf{R}, t)}
$$

with generating function $\chi=-\mathbf{A}(\mathbf{R}, t) \mathbf{R}$ and assume an initial Coulomb gauge of $\nabla_{\mathbf{R}} \mathbf{A}(\mathbf{R}, t)=$ 0 , the Schrödinger equation becomes

$$
i \hbar \frac{\partial}{\partial t}|\Psi(t)\rangle=\left[\frac{\mathbf{P}^{2}}{2 m}+V_{0}(\mathbf{R})-q \mathbf{E}(\mathbf{R}, t) \mathbf{R}-\frac{q}{m} \mathbf{B}(\mathbf{R}, t) \mathbf{S}\right]|\Psi(t)\rangle,
$$

where $\mathbf{E}(\mathbf{R}, t)$ denotes the electric component of the external field. This is called the length gauge form of the Schrödinger equation because of the additional scalar potential $-q \mathbf{E}(\mathbf{R}, t) \mathbf{R}$ in the Hamiltonian. Another simplification is that the canonical momentum $\mathbf{P}$ equals the kinetic momentum in this gauge (since $\mathbf{A}(\mathbf{R}, t)=0$ ).

In this thesis, we use the so-called dipole approximation of the electromagnetic field: since the typical wavelengths that we employ are in the near-infrared range (e.g. around around $\lambda=800 \mathrm{~nm}$ ), we can neglect the spatial variations of the electromagnetic field in the vicinity of the atomic processes. In this approximation, we can neglect the contributions of the spin and the magnetic fields.

From now on, we use atomic units. In this system of units, the reduced Plank's constant $\hbar$, the elementary charge $e$, the electron mass $m_{e}$, and the electric constant $1 / 4 \pi \varepsilon_{0}$ are chosen to be of unity. Then, the usual form of the time-dependent Schrödinger equation (1.1) in coordinate representation is

$$
i \frac{\partial}{\partial t} \Psi(\mathbf{r}, t)=\left[-\frac{1}{2 \mu} \nabla^{2}+V_{0}(\mathbf{r})+\mathbf{E}(t) \mathbf{r}\right] \Psi(\mathbf{r}, t),
$$


where $\mu$ is the mass of the particle measured in electron mass, and we made the substitution $q=-1$, that is by the electron's charge. Then we choose the polarization and the form of $\mathbf{E}(t)$ to complete the description.

The Hamiltonian of (1.5) is actually of the form that describes single-electron atoms or ions, if $V_{0}(\mathbf{r})$ set to be the $-Z /|\mathbf{r}|$ atomic binding Coulomb potential with core charge of $Z$. An important approximation for the solution is the so-called strong-field approximation $[19,21$, 58], where the atomic potential is treated as a perturbation, and the ionized wave packets are taken into account as Volkov states in the continuum. For a more accurate description numerical simulations are necessary, however.

\subsubsection{Three-dimensional model equation in cylindrical coordinates}

In typical strong-field applications one assumes a linearly polarized laser pulse, for which the motion can be naturally treated in cylindrical coordinates. We choose the polarization axis as the $z$-axis, and the electric field function as $\mathbf{E}(t)=\mathscr{E}_{z}(t) \hat{\varepsilon}_{z}$.

Let us consider the axially symmetric three-dimensional (3D) time-dependent Schrödinger equation in the cylindrical coordinates $\rho=\sqrt{x^{2}+y^{2}}$ and $z$ :

$$
i \frac{\partial}{\partial t} \Psi(z, \rho, t)=-\frac{1}{2 \mu}\left[\frac{\partial^{2}}{\partial z^{2}}+\frac{\partial^{2}}{\partial \rho^{2}}+\frac{1}{\rho} \frac{\partial}{\partial \rho}\right] \Psi(z, \rho, t)+V(z, \rho, t) \Psi(z, \rho, t) .
$$

The potential is the sum of an atomic binding potential (centered in the origin) and an interaction energy term. For a single active electron and the linearly polarized laser pulse, this interaction term in dipole approximation and in length gauge reads as:

$$
V(z, \rho, t)=V_{0}(z, \rho)+V_{\mathrm{ext}}(z, t), \text { and } V_{\mathrm{ext}}(z, t)=\mathscr{E}_{z}(t) \cdot z
$$

We also introduce the notation here for the kinetic energy operators:

$$
T_{z}=-\frac{1}{2 \mu} \frac{\partial^{2}}{\partial z^{2}}, \text { and } T_{\rho}=-\frac{1}{2 \mu}\left[\frac{\partial^{2}}{\partial \rho^{2}}+\frac{1}{\rho} \frac{\partial}{\partial \rho}\right]
$$

Here, we also assume that if $t \leq 0$ then $\mathscr{E}_{z}(t)=0$. Thus, the system is initially only subject to the binding potential, and the particle is in one of the bound states of the binding potential, which forms the initial state for the partial differential equation (1.6). Then the problem is treated as an initial value problem as $\Psi(z, \rho, 0)=\psi(z, \rho)$ is known. For this initial state and energy of a single-electron model atom a natural choice is the ground state the Coulomb problem $[57,59]$

$$
V_{0}(z, \rho)=-\frac{Z}{\sqrt{z^{2}+\rho^{2}}}, \quad E_{0}=-\frac{\mu Z^{2}}{2}, \quad \psi_{100}(z, \rho)=\sqrt{\frac{\mu^{3} Z^{3}}{\pi}} e^{-\mu Z \sqrt{\rho^{2}+z^{2}}}
$$

Regarding the boundary conditions, because of the singularity in the curvilinear coordinate $\rho$, Neumann boundary condition is to be posed along the line $\rho=0$ if $V_{0}(z, \rho)$ is $\operatorname{smooth}^{1}$. In

\footnotetext{
${ }^{1}$ In case of the Coulomb potential, we could assume the particular potential form of $V_{0}(z, \rho)=$ $-Z\left(z^{2}+\rho^{2}+a\right)^{-1 / 2}$ where $a$ is a very large, but finite number.
} 
order to find this boundary condition, we multiply (1.6) by $\rho$ and take the limit $\rho \rightarrow 0$ :

$$
\left.\frac{\partial \Psi}{\partial \rho}\right|_{\rho=0}=2 \mu \lim _{\rho \rightarrow 0}(\rho V(z, \rho, t) \Psi(z, \rho, t))=0 .
$$

Then $\Psi(z, \rho, t)$ is continuous and continuously differentiable in the $z-\rho$ plane and it has extremal value in $\rho=0$. The symmetry condition $\Psi(z, \rho, t)=\Psi(z,-\rho, t)$ also holds in this case. In case of the singular Coulomb potential (1.9), however, along the line $z=0$ a Robin type boundary condition is to be posed which we derive in Section 4.1.1 in this thesis.

The atomic system that we use in most of the cases is the hydrogen atom, or the singleactive-electron approximation (SAE) of certain noble gas atoms. This is widely adopted in strong-field models [19], where instead of multiple electrons we describe the motion with only a single electron that is in an effective atomic potential. This means, for example, using the properties of the Coulomb problem of (1.9) that one should change the core charge $Z$ to set the system's ground state energy to be the same as the experimental ionization potential $I_{P}$. For neon atoms these effective parameters are

$$
Z_{\mathrm{Ne}}^{(\mathrm{SAE})}=1.25929 \quad \text { and } \quad E_{0, \mathrm{Ne}}^{(\mathrm{SAE})} \approx-0.792905 .
$$

The most common application of this model is the calculation of the high-order harmonic spectra.

\subsubsection{One-dimensional modeling}

Even though the full solution of (1.6) is preferable to deduce the physical properties, it is sometimes a valid choice to approximately model the strong-field phenomena in one dimension (1D) when the external field is linearly polarized. Then the solution is obtained from the following 1D time-dependent Schrödinger equation

$$
i \frac{\partial}{\partial t} \Psi^{1 \mathrm{D}}(z, t)=\left[H_{0}^{1 \mathrm{D}}+V_{\mathrm{ext}}(z, t)\right] \Psi^{1 \mathrm{D}}(z, t)
$$

where the form of the external potential $V_{\text {ext }}(z, t)$ is given in (1.7). Note that this one-dimensional equation is completely analogous to Eq. (1.6), but without transverse degrees of freedom. Here, it is customary to assume a 1D atomic Hamiltonian of the following form:

$$
H_{0}^{1 \mathrm{D}}=T_{z}+V_{0}^{1 \mathrm{D}}(z)
$$

where $V_{0}^{1 \mathrm{D}}(z)$ is an atomic model potential of choice, which is traditionally chosen to qualitatively investigate specific features of the strong-field ionization.

There are a number of well-known 1D atomic model potentials in the literature [26, 29-31], having their advantages and disadvantages. Here we summarize the basics of some of these, which we think to be the most important for the modeling of strong-field phenomena. 
The soft-core Coulomb potential is defined as

$$
V_{0, \mathrm{Sc}}^{1 \mathrm{D}}(z)=-\frac{Z}{\sqrt{z^{2}+\alpha_{\mathrm{Sc}}^{2}}}
$$

where the smoothing parameter $\alpha_{\mathrm{Sc}}$ is usually adjusted to fit the ground state energy to a selected single-electron energy. For $\mu=1, Z=1$, and $\alpha_{\mathrm{Sc}}^{2}=2$, its ground state energy and ground state can be used as a $1 \mathrm{D}$ model hydrogen atom:

$$
E_{0, \mathrm{Sc}}=-\frac{1}{2}, \quad \psi_{0, \mathrm{Sc}}(z)=\mathscr{N}_{\mathrm{Sc}}\left(1+\sqrt{z^{2}+2}\right) e^{-\sqrt{z^{2}+2}}
$$

where $\mathscr{N}_{\mathrm{Sc}}$ is the normalization factor. The most important features of this model potential are that it is a smooth function, it has an asymptotic Coulomb form and positive energy Rydberg continuum. We can regard the form of this potential a more physical one which arises naturally from the Coulomb potential in cylindrical coordinates by replacing $\rho^{2}$ by $\alpha^{2}$ excluding the point of the singularity. For completeness, the energy of its first excited state of (1.14) is $E_{1, \mathrm{Sc}}=-0.2329034$. The main disadvantage of the soft-core Coulomb potential in a strongfield simulation is that it binds the electron too weakly, compared to the real 3D case.

The next 1D model potential is the 1D Dirac-delta potential $[32,33]$

$$
V_{0, \mathrm{DD}}^{1 \mathrm{D}}(z)=-Z \delta(z)
$$

which has the following ground state energy and ground state:

$$
E_{0, \mathrm{DD}}=-\frac{\mu Z^{2}}{2}, \quad \psi_{0, \mathrm{DD}}(z)=\sqrt{\mu Z} e^{-\mu Z|z|}
$$

The singularity of $V_{0, \mathrm{DD}}^{1 \mathrm{D}}(z)$ at $z=0$ is sometimes considered as a disadvantage, but this potential has its ground state with the same exponential form and cusp, and ground state energy as that of the 3D Coulomb problem which is intrinsically linked to the boundary condition at $z=$ 0 . A further advantage of this potential is that it makes certain analytical calculations easier e.g. in connection with tunnel ionization. We also used this type of model potential in our earlier publication [A1]. The main disadvantage of the 1D Dirac-delta potential in a strongfield simulation is that it binds the electron too strongly, compared to the real 3D case.

An additional naive choice would be to use simply the 1D singular Coulomb $-1 /|z|$ potential as a model potential, but unfortunately this system has a different behavior compared to the 3D case [60], because we must use $\psi(z=0)=0$ (particle-in-a-box) boundary condition at the point of the singularity, which also inhibits the wave function to cross the singularity. This feature can be remedied if we use the $1 \mathrm{D}$ regularized Coulomb potential

$$
V_{0, \mathrm{C}}^{1 \mathrm{D}}(z)=-\frac{Z}{|z|+a}
$$

where the value of the parameter $a$ is determined by requiring that the ground state energy is $E_{0, \mathrm{C}}=-\frac{\mu Z^{2}}{2}$. This model was investigated by Loudon [61] in relation with a one-dimensional hydrogen atom, the electron of which is bond by the singular 1D Coulomb potential. This 
regularized Coulomb potential in the singular limit of $a \rightarrow 0$ has the same energy levels and similar radial type eigenfunctions as the true $3 \mathrm{D}$ Coulomb problem.

Despite these positive features, the experience shows that (1.14) - (1.18) do not give strongfield simulation results that would be quantitatively comparable to those of the reference $3 \mathrm{D}$ system (cf. [31, 62]) therefore the model system parameters need to be manually adjusted, for example by changing the strength of $V_{\text {ext }}(z, t)$.

\subsection{Strong-field ionization of the interacting electron - ion- core system}

In this section we develop a more complete model of strong-field ionization of single-electron atoms, which involves treating the atom as an interacting electron - ion-core system. This formalism will introduce nonseparability between these two particles. This description does not invalidate the models and equations in the preceding section: the respective form of the time-dependent Schrödinger equation arise naturally.

The quantum mechanical description as an interacting two-body system of a hydrogen atom, or any other atom in the single-active-electron approximation, driven by a strong laser pulse, is naturally carried out as a two-body (or bipartite) problem consisting of the electron $(e)$ - ioncore $(c)$ system. Using the length gauge and dipole approximation developed in Section 1.2.1, we infer the following Hamiltonian for the composite system:

$$
\hat{H}_{e c}=\frac{\mathbf{P}_{e}^{2}}{2 m_{e}}+\frac{\mathbf{P}_{c}^{2}}{2 m_{c}}+V_{0}\left(\mathbf{R}_{e}-\mathbf{R}_{c}\right)+\mathbf{E}(t)\left(\mathbf{R}_{e}-\mathbf{R}_{c}\right),
$$

where $m_{e}(=1)$ and $m_{c}$ are the electron and the ion-core masses, respectively.

As it is well-known, this problem can be simplified by performing a coordinate transformation to the center-of-mass $\left(\mathbf{R}_{0}, \mathbf{P}_{0}\right)$ and relative coordinates $(\mathbf{R}, \mathbf{P})$ as

$$
\begin{aligned}
\mathbf{R}_{0}=\alpha_{e} \mathbf{R}_{e}+\alpha_{c} \mathbf{R}_{c}, & \mathbf{P}_{0}=\mathbf{P}_{e}+\mathbf{P}_{c} \\
\mathbf{R}=\mathbf{R}_{e}-\mathbf{R}_{c}, & \mathbf{P}=\alpha_{c} \mathbf{P}_{e}-\alpha_{e} \mathbf{P}_{c}
\end{aligned}
$$

where

$$
\alpha_{e}=m_{e} / M, \alpha_{c}=m_{c} / M, M=m_{e}+m_{c} .
$$

Using also the reduced mass $\mu=m_{e} m_{c} / M$, we obtain the Hamiltonian

$$
\hat{H}_{e c}=\frac{\mathbf{P}_{0}^{2}}{2 M}+\frac{\mathbf{P}^{2}}{2 \mu}+V_{0}(\mathbf{R})+\mathbf{E}(t) \mathbf{R},
$$

which becomes separable in these coordinates. Thus, in coordinate representation, the total wave function is given by

$$
\Psi_{e c}\left(\mathbf{r}_{e}, \mathbf{r}_{c}, t\right)=\Psi(\mathbf{r}, t) \Psi_{0}\left(\mathbf{r}_{0}, t\right),
$$

where the coordinates of the two sides are connected via the transformation (1.20). The time propagation can be performed in these coordinates independently, which we outline in the fol- 
lowing.

The center-of-mass part of the Hamiltonian describes a free-particle propagation via the time-dependent Schrödinger equation

$$
i \frac{\partial}{\partial t} \Psi_{0}\left(\mathbf{r}_{0}, t\right)=-\frac{1}{2 M} \nabla_{0}^{2} \Psi_{0}\left(\mathbf{r}_{0}, t\right)
$$

Any particular wave function that satisfies (1.24) can be chosen. For example, it can be a localized wave packet or plane wave.

The time evolution of the relative part in coordinate representation is given by the timedependent Schrödinger equation (1.6), which describes the strong-field ionization of a fictitious particle with mass $\mu=m_{e} m_{c} / M$. If we choose a linearly polarized laser field in direction $z$, the problem reduces to performing strong-field simulations in cylindrical coordinates with atomic Coulomb potential or a one-dimensional simulation with a 1D model potential as was discussed in Section 1.2.2 and 1.2.3. In order to address any possible ambiguity regarding the notation of $\Psi(\mathbf{r}, t)$, we would like to clarify the following: this relative coordinate part always incorporates all the strong-field ionization effects of a single-electron atom, that is acquired by numerical simulations in this thesis. Since even for hydrogen atom $\mu \approx 1$, i.e. $\mu$ is near the electron mass, it is known that the probability density and probability current of this relative wave function closely yield the physical quantities of the electrons' motion by itself (relative to the center-ofmass). However, it is not necessarily true that the relative wave function describes the actual quantum mechanical state of the electron. Thus, if we just simply call it the wave function of the electron, it is in always of the context of infinite nucleus mass of $M=\infty$, and $\mu=1$. This means that the relative wave function can give only an incomplete description of the electron's quantum mechanical state.

To summarize this section, while it is true that this problem separates in the center-of-mass reference frame and certain physical predictions of the relative wave function are close to that of the electron's, the electron and ion-core particles are linked through the nonseparable atomic potential, which creates quantum entanglement between the individual particles of the system.

\subsection{Characterization of the strong-field ionization}

In this section, we briefly summarize certain aspects that allow us the characterization of the strong-field process. Besides the intensity and the pulse shape of the laser field, the ionization process can be characterized by ionization type. For the actual time-dependent motion itself, we adopt the description by integrated time-dependent physical quantities and mean values.

\subsubsection{Ionization types}

Let us discuss the properties of the ionization process. We can categorize the ionization regarding which types of ionization channels are available: vertical or horizontal ionization channels [21]. First, we assume an external field of the form

$$
\mathbf{E}(t)=\hat{\varepsilon}_{z} \mathscr{E}_{z}(t) \quad \text { with } \quad \mathscr{E}_{z}(t)=F f(t) \cos (\omega t)
$$


where $F$ is the parameter of the strength of the external electric field and $f(t)$ gives the pulse shape which is scaled so that its minima are 0 and its maxima are 1.

The vertical ionization channel is also called multiphoton ionization. In the semiclassical picture, this ionization is attributed to the periodic distortions of the binding potential caused by the oscillating electric field. During each of these oscillations the bound electronic wave packet is "shaken up", absorbing energy, and gaining momentum up to the point in which it is collected enough to be able to cross the potential barrier. The prevalence of this ionization channel is important when the laser's period $T=2 \pi / \omega$ is not too large compared to the electronic response time of $2 \pi \hbar / E_{0}$, when the electron cannot adiabatically adjust to the distorted potential. However, if the pulse shape $f(t)$ is too narrow, i.e. it contains few cycles, this ionization channel is suppressed - the bound wave function has not enough time to absorb energy in this way. For weaker fields the perturbative calculations were a great success, and they showed that in the respective intensity regime the transition matrix elements from the ground state to continuum states with well defined momentum depend explicitly on energy difference $n \hbar \omega$, where $n$ is larger than a threshold number $n_{0}$. Thus, even in the semiclassical picture, we can identify $\hbar \omega$ as the photon energy, $n$ is the number of photons absorbed. Because of this nature of transition lines, this ionization channel is called multiphoton ionization. It is relatively inefficient since the transition matrix elements show $\sim F^{2 n}$ dependence, the exponent of which decreases at high intensities [63].

The horizontal ionization channel can be categorized regarding the electric field strength parameter $F$ : there is a specific value $F_{\text {tu }}$ that separates two regimes, in which the system has distinct behavior. In the tunneling ionization regime $F<F_{\mathrm{tu}}$ there is always a potential barrier $V(F, t)>E_{0}$ in the vicinity of the atom, while in the over-the-barrier ionization regime $F>F_{\mathrm{tu}}$ this barrier does vanish to a varying extent both in space and time, determined by $F$ and by the shape of the laser pulse. By solving for $z=z\left(E_{0}, F_{\mathrm{tu}}\right)$ in

$$
E_{0}=-\frac{1}{z}+z \cdot F_{\mathrm{tu}}
$$

at cross section $\rho=0$, a quick calculation reveals that this critical value is $F_{\text {tu }}=\left|E_{0}\right|^{2} / 4$, i.e. $F_{\mathrm{tu}} \approx 0.0624$ atomic units for hydrogen. It is important that in the over-the-barrier regime classical electron trajectories to the continuum are allowed at certain time instants, while in the tunneling regime the motion under the potential barrier is a quantum mechanical phenomena.

Now, assume that the electric field strength $F<F_{\text {tu }}$ is low enough. It was Keldysh [17] who first investigated the availability of the vertical and horizontal ionization channels (i.e. the multiphoton and the tunnel ionization regimes) for plane waves $(f(t)=1)$. He introduced the famous parameter of

$$
\gamma_{\mathrm{K}}=\sqrt{I_{P} / 2 U_{P}}, \text { where } U_{P}=F^{2} / 4 \omega^{2},
$$

which is now called the Keldysh parameter. Here, $I_{P}$ denotes the ionization potential of the atom and $U_{P}$ the average energy of the oscillating electrons (ponderomotive potential). According to this parameter traditionally we speak of tunneling ionization when $\gamma_{\mathrm{K}} \ll 1$ (i.e. a static field) and multiphoton ionization when $\gamma_{\mathrm{K}} \gg 1$. We note that this follows from that the Keldysh parameter can be written as $\gamma_{\mathrm{K}}=\omega \tau$, where $\tau$ is the Keldysh tunneling time [52, 64]. Thus, this 
parameter only characterizes the possible opportunities of electron tunneling during strong-field phenomena.

In this thesis we employ an external field with parameters $\omega$ and $F$ that yield $\gamma_{\mathrm{K}} \approx 1$, so various ionization channels will be present. In addition to this we employ only few-cycle nearinfrared laser pulses, neither the duration nor the angular frequency of which makes the multiphoton ionization to a leading contribution. Therefore we investigate the horizontal channels of ionization.

\subsubsection{Physical quantities}

Since the wave function $\Psi(\mathbf{r}, t)$ (which is the wave function of the relative part) encompasses all the effects of the external field, we can use certain physical quantities calculated only from it to describe some general features of the dynamics and ionization. For completeness, we give a limited list of the physical quantities here that can be used for characterizing this strong-field process, both in $1 \mathrm{D}$ and $3 \mathrm{D}$.

The first set of physical quantities calculated using the 1D reduced density from the 3D wave function which is given by

$$
\varrho_{z}^{3 \mathrm{D}}(z, t)=2 \pi \int_{0}^{\infty}|\Psi(z, \rho, t)|^{2} \rho \mathrm{d} \rho .
$$

In the $1 \mathrm{D}$ model, this is simply

$$
\varrho_{z}^{1 \mathrm{D}}(z, t)=\left|\Psi^{1 \mathrm{D}}(z, t)\right|^{2}
$$

The first interesting quantity is the $z$ component of the mean value $\langle\mathbf{r}\rangle$ that is

$$
\langle z\rangle(t)=\int_{-\infty}^{\infty} z \varrho_{z}(z, t) \mathrm{d} z
$$

and the mean velocity of this component

$$
\langle v\rangle_{z}(t)=\frac{\partial\langle z\rangle(t)}{\partial t}=\frac{1}{\mu} \operatorname{Im}\left\langle\Psi(t) \mid \partial_{z} \Psi(t)\right\rangle
$$

which can be also given as the average of the probability current density. These two give information about the kinematic properties of a "classical" particle that behaves according to the Ehrenfest's theorems. (We note that there can be no mean displacement in the transverse directions $x, y$ because of the dipole approximation we use.)

Another independent quantum mechanical marker is the standard root-mean-square deviation of $z$ as

$$
\sigma_{z}(t)=\sqrt{\left\langle z^{2}\right\rangle(t)-\langle z\rangle^{2}(t)}
$$

which gives information about the spatial extent of the ionized wave packets. Because the form of the Coulomb potential is spherically symmetric, the form of $\sigma_{x}(t)$ follows that of $\sigma_{z}(t)$.

An alternative descriptive time-dependent quantity we use is based on the projection onto 
the initial state as

$$
g(t)=1-|\langle\Psi(0) \mid \Psi(t)\rangle|^{2}
$$

which is actually the loss of the ground state population. This $g(t)$ is very close to the probability of leaving the vicinity of the center-of-mass $(\mathbf{r}=0)$ in typical strong-field processes. We have found that after the laser pulse it is a good indicator of the fraction of ionization and the continuum wave packets. This has been verified numerically in our actual calculations, and this feature of (1.33) was also utilized in other strong-field calculations like in the well-known Lewenstein model [19].

Another important physical quantity that we list here is related to the high-order harmonic generation [7, 10]. It is known that accelerating charges emit electromagnetic radiation, but on a single-atom level the calculation of this is not trivial, since the quantum description of the electron is neither a point charge, nor a charge distribution. However, if many similar atomic processes occur, the total emitted radiation will given by the coherent part of singleatom responses. Thus it is related to the average of the $z$ component of the particle acceleration which can be calculated simply as

$$
\left\langle a_{z}\right\rangle(t)=\frac{\partial\left\langle v_{z}\right\rangle(t)}{\partial t}
$$

Then the emitted spectrum is calculated from the dipole acceleration $\left\langle a_{z}\right\rangle$, and the power spectrum of which is given by

$$
p(f)=\left|\mathscr{F}\left[\left\langle a_{z}\right\rangle\right](f)\right|^{2}
$$

where $\mathscr{F}$ denotes the Fourier transform and $f$ is its frequency variable.

We can use the aforementioned physical quantities for the analysis of the strong-field dynamics, illustrating how much the atom was ionized and approximately in which direction the particle moves, and we can investigate the HHG spectrum using the Fourier transform of the dipole acceleration. 


\section{Chapter 2}

\section{Standard methods for the numerical solution of the time-dependent Schrödinger equation}

In this chapter we overview the relevant standard numerical methods that can be used for the real space numerical solution of the time-dependent Schrödinger equation (1.6). The numerical methods discussed here are advanced: the computational efficiency of the proper numerical solution of this particular partial differential equation is crucial in higher dimensions. In the following, we introduce the general approximations from which temporally and spatially highorder convergence of the solution can be achieved. We also discuss a particular high-order discretization of (1.6) within the Crank-Nicolson scheme.

\subsection{Crank-Nicolson method}

The Crank-Nicolson method discussed here will form the basis of the numerical equations in Chapter 4. In the following, we overview the relevant approximations of the time evolution operator, introducing the Crank-Nicolson form of the short-time evolution operator, then we introduce the finite difference discretizations applied to the cylindrical case, as mentioned earlier. We also discuss the numerical integration formula and the unitarity in the cylindrical case.

\subsubsection{Approximation of the time evolution operator}

The formal solution of (1.5) or (1.6) in terms of the time evolution operator $U\left(t, t^{\prime}\right)$ is the following

$$
U\left(t, t^{\prime}\right)=\mathscr{T} \exp \left[-i \int_{t^{\prime}}^{t} H\left(t^{\prime \prime}\right) d t^{\prime \prime}\right], \quad|\Psi(t)\rangle=U\left(t, t^{\prime}\right)\left|\Psi\left(t^{\prime}\right)\right\rangle .
$$

Here, the exponential operator is to be understood as a time-ordered quantity, which is a very difficult procedure if the Hamilton operators at different time instants do not commute. However, this is the case for the Hamiltonian we are considering.

To acquire suitable discretization in the time domain we have to approximate the $U(t, 0)$ time evolution operator directly. First, let us divide the time domain $[0, t]$, into $N_{t}$ equal subintervals of size $\Delta t$, then by the group property of $U(t, 0)$ we get 


$$
U(t, 0)=\prod_{k=0}^{N_{t}-1} U\left(t_{k+1}, t_{k}\right)
$$

where $t_{k}=k \Delta t$ and $\Delta t=t / N_{t}$. In one interval, we can write the evolution operator with its short-time form of

$$
U\left(t_{k+1}, t_{k}\right)=e^{-i \Delta t H_{k}}
$$

where $H_{k}$ is an effective time-independent Hamiltonian at the $k$ th step, which is related to the original one $H(t)$ by the Magnus expansion [65, 66]:

$$
H_{k}=\frac{1}{\Delta t} \int_{t_{k}}^{t_{k+1}} H\left(t^{\prime}\right) \mathrm{d} t^{\prime}+\frac{i}{2 \Delta t} \int_{t_{k}}^{t_{k+1}} \int_{t_{k}}^{t^{\prime}}\left[H\left(t^{\prime \prime}\right), H\left(t^{\prime}\right)\right] \mathrm{d} t^{\prime \prime} \mathrm{d} t^{\prime}+\ldots
$$

This includes infinitely many commutators of the Hamiltonians evaluated at different time points, to be integrated with respect to more and more variables. This series have to be approximated by cutting of the terms and approximating the integrals.

Using only the first term of this series, one can directly acquire the well-known second-order approximation of the Hamiltonian as

$$
H_{k}^{(2)}=H\left(t_{k+\frac{1}{2}}\right) \text {. }
$$

In order to have information about the next error term of the time evolution, we need to evaluate the Magnus commutator series to the fourth order. According to Puzynin et. al. [65], this improved approximation for a TDSE of the form (1.6) is

$$
H_{k}^{(4)}=\frac{1}{2 \mu}\left(-i \nabla+\frac{\Delta t^{2}}{12} \nabla \dot{V}\left(t_{k+1 / 2}\right)\right)^{2}+V\left(t_{k+1 / 2}\right)+\frac{\Delta t^{2}}{24} \ddot{V}\left(t_{k+1 / 2}\right) .
$$

where the top dots are the short hand notation for $\partial_{t}$ time derivatives. This formula is important even if we use only the second-order approximation, because the leading order of error depends on characteristics of the first and second time derivatives of $V(z, \rho, t)$.

For the exponential operators (2.3), first we consider the diagonal Padé-approximation of an exponential function

$$
e^{\lambda \cdot x}=\frac{{ }_{1} F_{1}(-M,-2 M, \lambda \cdot x)}{{ }_{1} F_{1}(-M,-2 M,-\lambda \cdot x)}+O\left(\lambda^{2 M+1}\right)
$$

where ${ }_{1} F_{1}$ is the confluent hypergeometric function, which in this case reduces to a polynomial of degree $M$ with real coefficients. This expression can be used for the exponential operators (2.3) with $\lambda=-i \Delta t$, and it can be shown that for self-adjoint operators the approximation is unitary [22].

From this, a generalized operator approximation scheme that is $\Delta t^{2 M+1}$ accurate can be obtained $[67,68]$ in a general form of

$$
e^{-i \Delta t H_{k}}=\prod_{s=1}^{M}\left[\left(1+i \frac{\Delta t}{x_{s}^{*}} H_{k}\right)^{-1}\left(1-i \frac{\Delta t}{x_{s}} H_{k}\right)\right]+O\left(\Delta t^{2 M+1}\right)
$$


where $x_{s}$ for $s=1, \ldots, M$ are the roots of the polynomial equation

$$
{ }_{1} F_{1}(-M,-2 M,-x)=0 \text {. }
$$

If we truncate both the Magnus-series at the first term in (2.4) and take a single coefficient of the Padé-approximation $(M=1)$, then we arrive at the second-order accurate implicit CrankNicolson scheme:

$$
\Psi\left(t_{k+1}\right)=\left(1+i H_{k} \frac{\Delta t}{2}\right)^{-1}\left(1-i H_{k} \frac{\Delta t}{2}\right) \Psi\left(t_{k}\right),
$$

which is called the Crank-Nicolson method. One can straightforwardly construct higher-order schemes using (2.8), yielding multiple implicit Crank-Nicolson substeps [67] in forms similar to (2.10). For time-dependent Hamiltonians though, a high-order accurate scheme should use the corresponding high-order effective Hamiltonian (2.4) for consistency.

\subsubsection{The cylindrical finite difference scheme}

The most widely employed approach to the solution of the time-dependent Schrödinger equation (1.5) for an atomic electron driven by a strong laser pulse is to use spherical polar coordinates: then the hydrogen eigenfunctions are analytic and one expands the wave function in $Y_{l, m}(\theta, \varphi)$ spherical harmonics with expansion coefficients $\phi_{l, m}(r, t)$ acting as radial or reduced radial functions, for which the problem is solved directly [69-73]. The usual drawback is that in order to calculate physical quantities either the real space reconstruction of the wave function is necessary or every observable must be given in this harmonic expansion. Also, the spherical grid of these methods is not well adapted to the electron's motion which is mainly along the polarization direction of the laser field. Therefore in some applications neither spherical coordinates, nor special basis functions are optimal, and one has to use Cartesian or cylindrical coordinate systems in numerical simulations.

The plan of using cylindrical coordinates demands an analytic boundary condition to be incorporated into the numerical solution of TDSE. This will constrain the applicable propagation methods: the singularity of the cylindrical radial coordinate at $\rho=0$ will introduce Neumann boundary conditions, overriding the Hamilton operator. As a consequence, typical explicit methods, for example staggered leapfrog [74, 75], second-order symmetric difference method [76, 77], any polynomial expansion method [76, 78] have been ruled out, leaving only implicit methods.

In the following, we continue by discretizing (2.10) in cylindrical coordinates to arrive at its discretized matrix form. For the actual simulations based on Eq. (1.6), it is necessary choose a finite spatial domain which is large enough to be able to contain the time-dependent wave function. Therefore, the wave function is defined within the interval $z \in\left[z_{\min }, z_{\max }\right], \rho \in$ $\left[0, \rho_{\max }\right]$, and additional boundary conditions are posed at three edges of the interval:

$$
\Psi\left(z>z_{\max }, \rho, t\right)=\Psi\left(z<z_{\min }, \rho, t\right)=\Psi\left(z,|\rho|>\rho_{\max }, t\right)=0,
$$

which are the boundary conditions of particle in a box i.e. a particle in an infinite potential well. In this region, we introduce the following particular form of the equidistant $2 \mathrm{D}$ spatial grid of 
the $z, \rho$ cylindrical coordinates:

$$
\begin{gathered}
z_{i}=z_{\min }+i \cdot \Delta z, \Delta z=\left(z_{\max }-z_{\min }\right) / N_{z}, i \in\left[0, N_{z}\right], \\
\rho_{j}=j \cdot \Delta \rho, \Delta \rho=\rho_{\max } / N_{\rho}, j \in\left[0, N_{\rho}\right]
\end{gathered}
$$

Our next step is to discretize the effective Hamiltonian $H_{k}$, and to construct the Hamiltonian matrix. Let us introduce the finite difference approximation of the Laplacian as $\nabla^{2} \approx \widetilde{L}_{z}+\widetilde{L}_{\rho}$, which we will incorporate into the discrete Hamiltonian $\widetilde{H}_{k}$. Based on Appendix A, highorder difference formulas can be calculated using one-dimensional Taylor-expansions centered around the points $z_{i}$ or $\rho_{j}$. Using the formulas (A.3) and (A.4) of this Appendix, the symmetric fourth-order accurate forms of $\widetilde{L}_{z}$ and $\widetilde{L}_{\rho}$ read as:

$$
\begin{gathered}
\widetilde{L}_{z} \Psi_{i, j}(t)=\frac{-\Psi_{i-2, j}+16 \Psi_{i-1, j}-30 \Psi_{i, j}+16 \Psi_{i+1, j}-\Psi_{i+2, j}}{12 \Delta z^{2}} \\
\widetilde{L}_{\rho} \Psi_{i, j}(t)=\frac{(-1+1 / j) \Psi_{i, j-2}+(16-8 / j) \Psi_{i, j-1}-30 \Psi_{i, j}+(16+8 / j) \Psi_{i, j+1}+(-1-1 / j) \Psi_{i, j+2}}{12 \Delta \rho^{2}}
\end{gathered}
$$

These fourth-order formulas are optimal in the sense that of using more than five points are can problematic here, because the higher-order the finite difference formula is, the higher-order derivatives of $\Psi(z, \rho, t)$ should be continuous.

Using the standard second-order accurate form (2.10) of the exponential operator with the discretized Laplacian in the Hamilton matrix, we get the following implicit scheme for all $i \in$ $\left[0, N_{z}\right]$ and $j \in\left[1, N_{\rho}\right]$ :

$$
\left(1+\alpha \beta \widetilde{L}_{z}+\alpha \beta \widetilde{L}_{\rho}+\alpha V_{i, j}\right) \Psi_{i, j}\left(t_{k+1}\right)=\left(1-\alpha \beta \widetilde{L}_{z}-\alpha \beta \widetilde{L}_{\rho}-\alpha V_{i, j}\right) \Psi_{i, j}\left(t_{k}\right),
$$

where $\alpha=i \Delta t / 2, \beta=-1 / 2 \mu$ and the potential is evaluated at the temporal midpoint $V_{i, j}=$ $V\left(z_{i}, \rho_{j}, t_{k+1 / 2}\right)$. The box boundary conditions $\Psi_{i, j}=0$ are applied if $j>N_{\rho}$ or $i>N_{z}$ or $i<0$.

Let us assume that the atomic potential $V_{0}(z, \rho)$ is smooth. To discretize the resulting Neumann boundary conditions (1.10) at $\rho=0$, we need a one-sided finite difference formula for the first derivative. Using the formula (A.9) of Appendix A, we introduce the following fourth-order accurate forward difference operator:

$$
\widetilde{D}_{\rho} \Psi_{i, j}(t)=\frac{-25 \Psi_{i, j}+48 \Psi_{i, j+1}-36 \Psi_{i, j+2}+16 \Psi_{i, j+3}-3 \Psi_{i, j+4}}{12 \Delta \rho}
$$

Then this gives the following implicit equations at $j=0$ for all $i \in\left[0, N_{z}\right]$ :

$$
\widetilde{D}_{\rho} \Psi_{i, 0}\left(t_{k+1}\right)=0
$$

For simplicity, let us introduce the short hand notations $X_{i, j}=\Psi_{i, j}\left(t_{k+1}\right), \Psi_{i, j}=\Psi_{i, j}\left(t_{k}\right)$, then by substituting the finite difference Laplacians (2.14), (2.15) into (2.16), we arrive at the 
following final form of linear equations for all $i \in\left[0, N_{z}\right], j \in\left[1, N_{\rho}\right]$ :

$$
\begin{gathered}
(-1+1 / j) \beta_{\rho} X_{i, j-2}+(16-8 / j) \beta_{\rho} X_{i, j-1}+(16+8 / j) \beta_{\rho} X_{i, j+1}+(-1-1 / j) \beta_{\rho} X_{i, j+2} \\
\quad-\beta_{z} X_{i-2, j}+16 \beta_{z} X_{i-1, j}+\left(1-30 \beta_{\rho}-30 \beta_{z}+\alpha V_{i, j}\right) X_{i, j}+16 \beta_{z} X_{i+1, j}-\beta_{z} X_{i+2, j} \\
=(1-1 / j) \beta_{\rho} \Psi_{i, j-2}+(-16+8 / j) \beta_{\rho} \Psi_{i, j-1}+(-16-8 / j) \beta_{\rho} \Psi_{i, j+1}+(1+1 / j) \beta_{\rho} \Psi_{i, j+2} \\
+\beta_{z} \Psi_{i-2, j}-16 \beta_{z} \Psi_{i-1, j}+\left(1+30 \beta_{\rho}+30 \beta_{z}-\alpha V_{i, j}\right) \Psi_{i, j}-16 \beta_{z} \Psi_{i+1, j}+\beta_{z} \Psi_{i+2, j},
\end{gathered}
$$

where

$$
\alpha=i \Delta t / 2, \beta=-1 / 2 \mu, \beta_{\rho}=\alpha \beta /\left(12 \Delta \rho^{2}\right), \beta_{z}=\alpha \beta /\left(12 \Delta z^{2}\right) .
$$

The equations forced by the Neumann conditions for all $i \in\left[0, N_{z}\right]$ from (2.17), (2.18) are

$$
-25 X_{i, 0}+48 X_{i, 1}-36 X_{i, 2}+16 X_{i, 3}-3 X_{i, 4}=0
$$

The spatial discretization in the cylindrical coordinate system and the Neumann boundary conditions make the unitarity of the algorithm, and in a broader sense, the accuracy of spatial integrations a more subtle issue than usual. One has to find an appropriate discrete inner product formula that is conserved at least with the accuracy of the finite differences, and which can be evaluated with sufficient accuracy using the cylindrical grid. We give a solution to this auxiliary problem in the next section.

Although it corresponds to a 3D propagation, we call this scheme 2D Crank-Nicolson method because it involves only two spatial coordinates. This is already a correct propagation algorithm by itself, however, it suffers from the numerically inefficient solution of the resulting linear equations: if we combine the $i, j$ indices into a single one (by flattening the two-dimensional array) as $l=i \cdot\left(N_{\rho}+1\right)+j$, we obtain a block pentadiagonal matrix of size $\left(N_{z}+1\right)^{2}\left(N_{\rho}+1\right)^{2}$, with block size $\left(N_{\rho}+1\right)^{2}$. Inverting this type of matrix is computationally intense $[79,80]$ because the width of the diagonal is $4 N_{\rho}+1$ : despite its apparent simplicity, the numerical cost of this task is $\sim N_{z} N_{\rho}^{3}$ which is prohibits performing actual strong-field simulations. These facts inspired us to develop an improved algorithm which has almost all advantages of this 2D Crank-Nicolson method but needs much less numerical effort.

For completeness, we note that one-dimensional Crank-Nicolson schemes where only the $z_{i}$ grid is present do not suffer this prohibitive computational load, the computational cost of a pentadiagonal scheme with the corresponding size is $\sim N_{z}$. The form of one-dimensional equations follow straightforwardly from (2.19) with $\beta_{\rho}=0$.

\subsubsection{Approximating the cylindrical inner product}

In the following, we seek a discretized representation of the inner product formula in the cylindrical coordinate system as

$$
\langle\Phi \mid \Psi\rangle=2 \pi \int_{-\infty}^{+\infty} \int_{0}^{\infty} \rho \Phi^{*}(z, \rho) \Psi(z, \rho) \mathrm{d} \rho \mathrm{d} z=\sum_{i, j} c_{i, j} \Phi_{i, j}^{*} \Psi_{i, j}
$$

The naive approach with coefficients $c_{i, j}=2 \pi \rho_{j} \Delta \rho \Delta z$ causes inaccuracy which originates 
solely from the $\rho=0$ edge and its neighborhood, because the formula with this particular $c_{i, j}$ has exponential convergence at the box boundaries [79].

Besides the accuracy, the conservation of a discretized scheme of form (2.22) is also an issue: given a discrete Hamiltonian matrix $H$ in the Padé-approximation then the time evolution will be unitary with respect to the inner product $\sum_{i, j} c_{i, j} \Phi_{i, j}^{*} \Psi_{i, j}$ only if $H$ is self-adjoint. Unfortunately, because of the boundary conditions at $\rho=0$ the Hamiltonian matrix $H$ does not exist on the $\rho=0$ line, therefore the standard norm of form $\sum_{i, j} c_{i, j} \Psi_{i, j}^{*} \Psi_{i, j}$ is not perfectly conserved by the numerical Crank-Nicolson scheme.

Therefore, our aim is to approximate (2.22) with an order that is same or higher than the order of the finite difference scheme. To achieve this goal, we use Lagrange [79] interpolating polynomial $p_{i, j}(\rho)$ defined on points $\left(\rho_{j}, Q_{i, j}\right),\left(\rho_{j+1}, Q_{i, j+1}\right),\left(\rho_{j+2}, Q_{i, j+2}\right),\left(\rho_{j+3}, Q_{i, j+3}\right)$, $\left(\rho_{j+4}, Q_{i, j+4}\right),\left(\rho_{j+5}, Q_{i, j+5}\right),\left(\rho_{j+6}, Q_{i, j+6}\right)$ for a given $z_{i}$ line, where $Q_{i, j}$ is the integrand in $\langle\Phi \mid \Psi\rangle=\iint Q(z, \rho) \mathrm{d} \rho \mathrm{d} z$. We use an elementary integral formula between $\rho_{j}$ and $\rho_{j+1}$ :

$$
\begin{aligned}
\int_{\rho_{j}}^{\rho_{j+1}} Q(z, \rho) \mathrm{d} \rho= & {\left[\frac{19087}{60480} Q_{i, j}+\frac{2713}{2520} Q_{i, j+1}-\frac{15487}{20160} Q_{i, j+2}+\frac{586}{945} Q_{i, j+3}-\frac{6737}{20160} Q_{i, j+4}\right.} \\
& \left.+\frac{263}{2520} Q_{i, j+5}-\frac{863}{60480} Q_{i, j+6}\right] \cdot \Delta \rho+O\left(\Delta \rho^{8}\right) .
\end{aligned}
$$

We sum up this for all $i, j$ points, and utilize the boundary conditions for $j \geq N_{\rho}$, then we arrive at the following integral formula, which is our choice to approximate the scalar product (2.22):

$$
\begin{aligned}
\langle\Phi \mid \Psi\rangle= & \sum_{i=0}^{N_{z}}\left[\frac{19087}{60480} \rho_{0} \Phi_{i, 0}^{*} \Psi_{i, 0}+\frac{84199}{60480} \rho_{1} \Phi_{i, 1}^{*} \Psi_{i, 1}+\frac{18869}{30240} \rho_{2} \Phi_{i, 2}^{*} \Psi_{i, 2}+\frac{37621}{30240} \rho_{3} \Phi_{i, 3}^{*} \Psi_{i, 3}\right. \\
& \left.+\frac{55031}{60480} \rho_{4} \Phi_{i, 4}^{*} \Psi_{i, 4}+\frac{61343}{60480} \rho_{5} \Phi_{i, 5}^{*} \Psi_{i, 5}+\sum_{j=6}^{N_{\rho}} \rho_{j} \Phi_{i, j}^{*} \Psi_{i, j}\right] \cdot 2 \pi \Delta \rho \Delta z
\end{aligned}
$$

This achieves the high integration accuracy (it is exact for a polynomial of $\rho$ up to degree 6), which is needed: the computed norm variations become proportional to $\Delta \rho^{4}$, which is consistent with the accuracy of the spatial finite differences. This does not affect the stability of the constructed Crank-Nicolson scheme.

For completeness, we note that in 1D the proper discrete inner product is simply

$$
\left\langle\Phi^{1 \mathrm{D}} \mid \Psi^{1 \mathrm{D}}\right\rangle=\sum_{i=0}^{N_{z}} \Phi_{i}^{1 \mathrm{D} *} \Psi_{i}^{1 \mathrm{D}} \Delta z
$$

and pose no such difficulties ${ }^{1}$ that exist in cylindrical coordinates.

\footnotetext{
${ }^{1}$ Assuming that $\Psi^{1 \mathrm{D}}$ and $\Phi^{1 \mathrm{D}}$ are smooth enough.
} 


\subsection{Operator splitting schemes}

As we have seen in the previous section, the 2D Crank-Nicolson scheme with the boundary condition (2.18) is a possible but ineffective way of solving the TDSE numerically. Now we are going to discuss how to apply the well-known method of operator splitting in order to simplify the related short-time exponential operators.

\subsubsection{Operator splitting formulas}

The approach of the operator splitting method is to factorize the exponential operator of form $e^{\lambda H}$ into multiple easy-to-solve parts. From the Taylor-expansion of the exponential operator

$$
e^{\lambda(A+B)}=\sum_{n=0}^{\infty} \frac{(A+B)^{n}}{n !} \lambda^{n},
$$

follow the two main formulas [81] which form the basis of the operator splitting schemes, namely the Baker-Campbell-Hausdorff formula:

$$
e^{\lambda A} e^{\lambda B}=e^{\lambda(A+B)+\lambda^{2} \frac{1}{2}[B, A]+\lambda^{3} \frac{1}{12}[A-B,[A, B]]}+O\left(\lambda^{4}\right),
$$

and the Zassenhaus formula

$$
e^{\lambda(A+B)}=e^{\lambda A} e^{\lambda B} e^{\lambda^{2} \frac{1}{2}[A, B]} e^{\lambda^{3} \frac{1}{6}[A+2 B,[A, B]]}+O\left(\lambda^{4}\right) .
$$

Both of these contain infinitely many commutators of $A$ and $B$. Of course, if $[A, B]=0$ then $e^{\lambda(A+B)}=e^{\lambda A} e^{\lambda B}$ exactly. As the above formulas suggest, the $O\left(\lambda^{4}\right)$ terms can be further factorized into the exponents. An extended analysis is available in Refs. [82, 83].

If one uses (2.27) and (2.28) to acquire a symmetric decomposition, only odd leading order of $\lambda$ will appear in the formula as the error term. This is a requirement for quantum propagation though, because the presence of even-order terms would destroy the unitary evolution of the wave function. A well-known unitary example is the widely used standard symmetric secondorder accurate formula (or Strang splitting, after [84]):

$$
e^{\lambda(A+B)}=e^{\lambda A / 2} e^{\lambda B} e^{\lambda A / 2}+C_{3} \lambda^{3}+O\left(\lambda^{4}\right),
$$

where $C_{3}$ is a combination of commutators of $A$ and $B$. A direct fourth-order splitting scheme was derived by Chin and Suzuki $[85,86]$ :

$$
e^{\lambda(A+B)}=e^{\lambda \frac{1}{6} A} e^{\lambda \frac{1}{2} B} e^{\lambda \frac{2}{3} A+\lambda^{3} \frac{1}{72}[A,[B, A]]} e^{\lambda \frac{1}{2} B} e^{\lambda \frac{1}{6} A}+C_{5} \lambda^{5}+O\left(\lambda^{6}\right) .
$$

This splitting requires also the evaluation of the $[A,[B, A]]$ commutator, which can rise additional difficulties, depending on the particular form of $A$ and $B$.

We proceed by introducing another kind of higher-order operator splitting, based on the work of Bandrauk and Shen [87], who developed an iterative method to improve the accuracy of the (2.29) scheme. Let us denote the second-order accurate form with $S_{2}(\lambda)=e^{\lambda A / 2} e^{\lambda B} e^{\lambda A / 2}$, 
then their iteration method reads for $n=4,6, \ldots$ as

$$
S_{n}(\lambda)=S_{n-2}(s \lambda) S_{n-2}((1-2 s) \lambda) S_{n-2}(s \lambda)+C_{n-1}\left(2 s^{n-1}+(1-2 s)^{n-1}\right) \lambda^{n-1}+O\left(\lambda^{n+1}\right)
$$

where the parameter $s$ must be for each iteration step a real root of the corresponding polynomial equation

$$
2 s^{n-1}+(1-2 s)^{n-1}=0 \text {. }
$$

In (2.31) only the odd error terms appear, because of the unitarity and the symmetry of the splitting scheme. So the $S_{n}(\lambda)$ requires $3^{n / 2}$ evaluations of $S_{2}$, in the worst case.

This scheme was already generalized for time-dependent Hamiltonians of the form $H(t)=$ $A(t)+B(t)$ in [87, 88] as follows. Inserting the second-order effective Hamiltonian (2.5) into the formula (2.29) with $\lambda=-i \Delta t$, the second-order accurate splitting of the evolution operator becomes

$$
U_{2}(t+\Delta t, t)=e^{-i \frac{\Delta t}{2} A(t+\Delta t / 2)} e^{-i \Delta t B(t+\Delta t / 2)} e^{-i \frac{\Delta t}{2} A(t+\Delta t / 2)} .
$$

Then (2.31) will take the form for $n=4,6 \ldots$

$U_{n}(t+\Delta t, t)=U_{n-2}(t+\Delta t, t+(1-s) \Delta t) U_{n-2}(t+(1-s) \Delta t, t+s \Delta t) U_{n-2}(t+s \Delta t, t)+O\left(\lambda^{n+1}\right)$

and $s$ being the same as in the time-independent case (2.32). We note here that interestingly, the fourth-order approximation $U_{4}$ in (2.34) decreases the error even if the time evolution governed by a nonlinear time-dependent Schrödinger equation, if the nonlinear error term is corrected in the formulation of the $U_{2}$ propagator [88, 89].

We can also write out an alternate form of iteration (2.34) for time-dependent Hamiltonians, by using the same principles, for $n=6,10, \ldots$

$$
\begin{aligned}
U_{n}(t+\Delta t, t)= & U_{n-4}(t+\Delta t, t+(1-s) \Delta t) U_{n-4}(t+(1-s) \Delta t, t+(1-s-p) \Delta t) \\
& \times U_{n-4}(t+(1-s-p) \Delta t, t+(s+p) \Delta t) \\
& \times U_{n-4}(t+(s+p) \Delta t, t+s \Delta t) U_{n-4}(t+s \Delta t, t)+O\left(\lambda^{n+1}\right),
\end{aligned}
$$

where $s, p$ must be the simultaneous real roots of equations

$$
2 s^{n-3}+2 p^{n-3}+(1-2 s-2 p)^{n-3}=0, \text { and } 2 s^{n-1}+2 p^{n-1}+(1-2 s-2 p)^{n-1}=0 .
$$

However, this formula for $S_{6}$ requires five evaluations of $S_{2}$, compared to nine in the case of Eq. (2.31).

Although the fourth-order formula (2.30) with the fourth-order effective Hamiltonian (2.6) seems to be superior compared to the iterative propagation (2.34) (because of the extra information given by the temporal and spatial commutators, and less evaluations), the schemes (2.34) and (2.35) do not require the calculation of commutators, they decrease all the $\Delta t$-dependent errors simultaneously and they are easy to implement. However, they involve backward time steps, which means they do not work very well with nonunitary problems. 


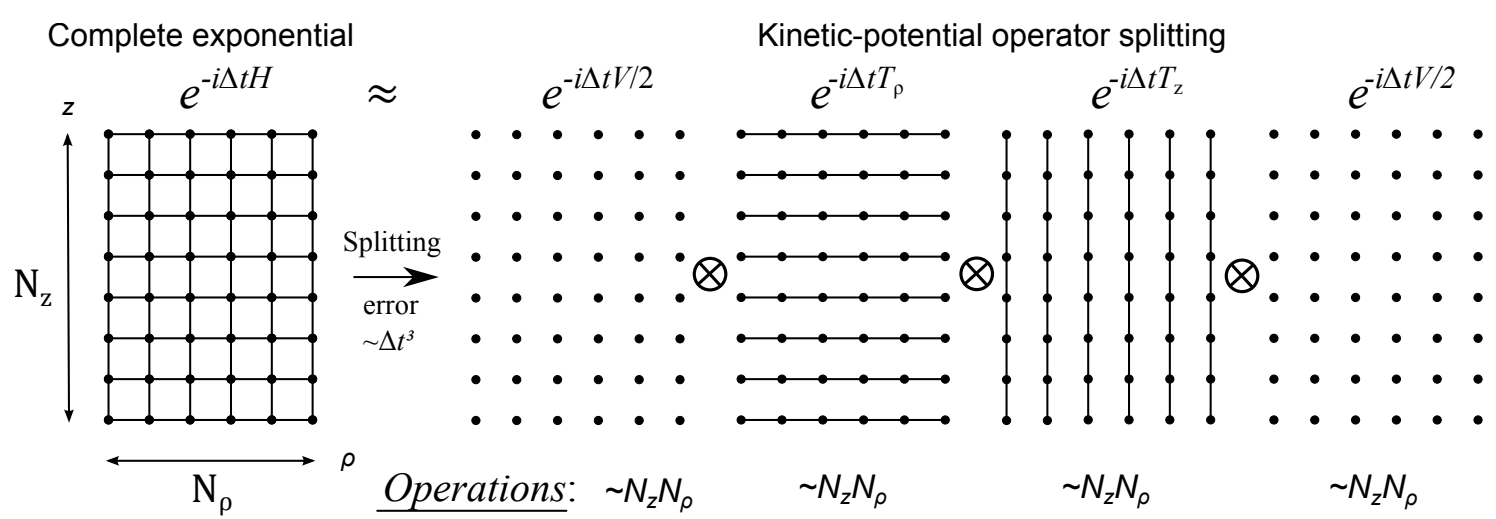

Figure 2.1: Sketch of the standard kinetic-potential splitting scheme (2.38). The solid lines represent coupling between the gridpoints of the exponential operator evaluations: we see that because of the kinetic energy operators $T_{\rho}$ and $T_{z}$ has been moved to different exponentials, the coupling has been split directionally. We also indicate the approximate operations count needed to apply the particular exponential operator in a split-step finite difference scheme.

Another class of high-order split-operator methods for diffusive parabolic partial differential equations was developed in [90], where the exponential operator is found by an ansatz of

$$
S_{2 n}(\lambda)=\sum_{k=1}^{n} c_{k} S_{2}^{k}\left(\frac{\lambda}{k}\right)+O\left(\lambda^{2 n+1}\right) \text { with } c_{k}=\prod_{j=1(\neq k)}^{n} \frac{c_{k}^{2}}{c_{k}^{2}-c_{j}^{2}} .
$$

Here $S_{2}$ is the same second-order formula which is used in (2.31). The fourth-order formula is simply given by $S_{4}(\lambda)=-\frac{1}{3} S_{2}(\lambda)+\frac{4}{3} S_{2}^{2}\left(\frac{\lambda}{2}\right)$. Thus, once $S_{2}$ is properly constructed, all high-order formulas (2.34), (2.35), (2.37) can be utilized immediately. The (2.37) method is suitable for the imaginary time propagation $[70,90,91]$ with $\lambda \rightarrow-\Delta t$, i.e. for determination of the lowest energy eigenstates of stationary potentials.

\subsubsection{Directional splitting of the exponential operator}

The most common way of factorizing the exponential operator $e^{\lambda H}$ is that the different spatial coordinate derivatives decouple into different exponential operators, i.e. to use a directional splitting. Then the propagation can be carried out by solving multiple one-dimensional TDSE$\mathrm{s}$ in succession. The most frequent realization of this is the so-called potential-kinetic term splitting (see Fig. 2.1), which is mainly used in conjunction with the Fourier-transformation methods in Cartesian coordinates [76, 92, 93]. However, if we apply the potential-kinetic term splitting to the cylindrical problem of (1.6) then we have to write Hamiltonian as $H=T_{\rho}+T_{z}+$ $V$ where $V$ is the potential, $T_{\rho}$ and $T_{z}$ are the kinetic energy operators of (1.8). The $[A,[B, A]]$ commutator will take a rather simple form of $\left[V,\left[T_{\rho}+T_{z}, V\right]\right]=|\nabla V|^{2} / \mu$. Thus, the direct second-order (2.29) and fourth-order (2.30) symmetric splitting schemes are written as

$$
\begin{gathered}
e^{\lambda\left(T_{\rho}+T_{z}+V\right)}=e^{\lambda V / 2} e^{\lambda T_{\rho}} e^{\lambda T_{z}} e^{\lambda V / 2}+C_{3} \lambda^{3}+O\left(\lambda^{4}\right) \\
e^{\lambda\left(T_{\rho}+T_{z}+V\right)}=e^{\lambda \frac{1}{6} V} e^{\lambda \frac{1}{2} T_{\rho}} e^{\lambda \frac{1}{2} T_{z}} e^{\lambda \frac{2}{3} V+\lambda^{3} \frac{1}{72} \frac{1}{\mu}|\nabla V|^{2}} e^{\lambda \frac{1}{2} T_{\rho}} e^{\lambda \frac{1}{2} T_{z}} e^{\lambda \frac{1}{6} V}+C_{5} \lambda^{5}+O\left(\lambda^{6}\right),
\end{gathered}
$$


where $\lambda=-i \Delta t$. We also note that these split-operator formulas by themself will introduce error, scaling as $\Delta t^{3}$ and $\Delta t^{5}$, compared to the stationary states of the exact time-independent Hamiltonian. In the case of a time-dependent Hamiltonian, the respective second-order (2.5) or fourth-order (2.6) effective Hamiltonians should be used. These truncations of the Magnusseries (2.4) will introduce additional $\Delta t^{3}$ - and $\Delta t^{5}$-dependent errors in time evolution.

The magnitudes of the aforementioned numerical errors will depend on the derivatives of $V(z, \rho, t)$ : in the case of external electric field the smoothness of $V$ in time is a reasonable assumption. On the other hand, the spatial dependence of the atomic potential should also behave the same way if we wish to use operator splitting. As we suspect, it will not always be the case, especially in atomic or molecular physics. We can deduce from (2.39) that the leading order of error of the second-order scheme must have a dependence of $\Delta t^{3}|\nabla V|^{2}$, e.g. the error characteristics strongly depend on the magnitude of the spatial derivatives of $V(z, \rho)$. In the case of an atomic $1 / r$ Coulomb potential this error term will be $\Delta t^{3} r^{-4}$ which becomes significant only in the region $r<1$, where it is increasing rapidly with fourth power of $1 / r$, and the split-operator scheme completely breaks down at $r=0$. This also illustrates the fact that nondifferentiability of either the potential or the wave function could potentially make operator splitting like (2.38), (2.39) less-than-useful.

Up to now, we have seen that directional spitting related exponential operator factorizations result drastic speed improvement for well-behaved potentials: we can directly apply the fivepoint finite difference Crank-Nicolson method to the $e^{\lambda T_{z}}$ and $e^{\lambda T_{\rho}}$ exponents for evaluation, thus we have to solve for locally decoupled one-dimensional wave functions. This is known as the split-step finite difference method $[94,95]$. Then, the approximate operations count of evaluating the formula (2.38) is $\sim N_{z} N_{\rho}$, which is smaller by the factor of $N_{\rho}^{2}$ compared to the full Crank-Nicolson problem of the same size. On the other hand, the full Crank-Nicolson scheme is able to incorporate the Neumann boundary conditions at $\rho=0$ into the implicit linear equations properly, and it does not suffer from the catastrophic error blow up while we approach the origin in the case of the Coulomb potential. 


\section{CHAPTER 3}

\section{Theoretical description of entangled quantum systems}

Quantum entanglement is probably the most fundamental of the quantum features and is a purely nonclassical correlation: if the spatial motion of two particles, for example, the electron and its parent ion-core is entangled (i.e. their positions or momenta), then any measurement made on one of these particles will affect measurements on the other particle, even when the interaction term in their Hamiltonian is negligible between them. In this chapter we overview the standard approach of quantifying quantum entanglement between these two particles. We will also introduce elements of quantum informational theory which can quantify the quantum correlation information between two arbitrary systems generally.

\subsection{Quantification of bipartite entanglement}

In the following, we recall the standard theory of quantum entanglement for pure state bipartite systems, emphasizing the features specific to the features of continuous variable quantum entanglement [38], where the quantum states described by square-integrable coordinate wavefunctions of infinite-dimensional Hilbert spaces. We introduce the concept of density matrices and quantum entropies, which quantify the quantum entanglement in the standard case.

\subsubsection{Schmidt decomposition}

Let us introduce two systems $e$ and $c$, which are two distinguishable particles, for example, the electron and its parent ion-core. We assume that the composite system $e c$ is a closed quantum system in a pure state represented by the wave function $\Psi_{e c}\left(\mathbf{r}_{e}, \mathbf{r}_{c}, t\right)$. The two subsystems $e$ and $c$ are entangled if $\Psi_{e c}$ is not separable with respect to the coordinates of these subsystems:

$$
\Psi_{e c}\left(\mathbf{r}_{e}, \mathbf{r}_{c}, t\right) \neq \Psi_{e}\left(\mathbf{r}_{e}, t\right) \Psi_{c}\left(\mathbf{r}_{c}, t\right)
$$

It is well-known that then the result of the measurement of subsystem $e$ affects the outcome of measurements on subsystem $c$ and vice-versa. That is, performing measurement on either particle changes the other particle's quantum state in a nonlocal manner.

To quantify this entanglement, we need the relevant concept of density matrices: the quan- 
tum state of the composite system is described by the two-particle pure state density matrix:

$$
\varrho_{e c}\left(\mathbf{r}_{e}^{\prime}, \mathbf{r}_{e}, \mathbf{r}_{c}^{\prime}, \mathbf{r}_{c}, t\right)=\Psi_{e c}^{*}\left(\mathbf{r}_{e}^{\prime}, \mathbf{r}_{c}^{\prime}, t\right) \Psi_{e c}\left(\mathbf{r}_{e}, \mathbf{r}_{c}, t\right)
$$

and the single-particle density matrices are obtained by tracing over the other particle's degrees of freedom. The reduced single-particle ion-core density matrix is

$$
\varrho_{c}\left(\mathbf{r}_{c}^{\prime}, \mathbf{r}_{c}, t\right)=\operatorname{Tr}_{e}\left[\varrho_{e c}\right]=\int \varrho_{e c}\left(\mathbf{r}_{e}, \mathbf{r}_{e}, \mathbf{r}_{c}^{\prime}, \mathbf{r}_{c}, t\right) \mathrm{d} \mathbf{r}_{e}^{3}
$$

and the reduced single-particle electron density matrix is

$$
\varrho_{e}\left(\mathbf{r}_{e}^{\prime}, \mathbf{r}_{e}, t\right)=\operatorname{Tr}_{c}\left[\varrho_{e c}\right]=\int \varrho_{e c}\left(\mathbf{r}_{e}^{\prime}, \mathbf{r}_{e}, \mathbf{r}_{c}, \mathbf{r}_{c}, t\right) \mathrm{d} \mathbf{r}_{c}^{3} .
$$

These quantities contain every quantum information about the respective single-particle properties, and they are directly related to the entanglement information we need. To show this, we refer to the Schmidt theorem [96,97], which states that there exists a unique decomposition of the entangled wave function $\Psi_{e c}$ of the bipartite system $e c$ into a sum of the following form:

$$
\Psi_{e c}\left(\mathbf{r}_{e}, \mathbf{r}_{c}, t\right)=\sum_{k} \sqrt{\lambda_{k}(t)} \phi_{k}\left(\mathbf{r}_{c}, t\right) \psi_{k}\left(\mathbf{r}_{e}, t\right)
$$

where $\phi_{k}\left(\mathbf{r}_{c}, t\right)$ and $\psi_{k}\left(\mathbf{r}_{e}, t\right)$ are orthonormal basis functions in the respective spaces. They are acquired after the diagonalization of the single-particle reduced density matrices (3.3) and (3.4) as

$$
\begin{aligned}
& \varrho_{e}\left(\mathbf{r}_{e}^{\prime}, \mathbf{r}_{e}, t\right)=\sum_{k} \lambda_{k}(t) \psi_{k}^{*}\left(\mathbf{r}_{e}^{\prime}, t\right) \psi_{k}\left(\mathbf{r}_{e}, t\right), \\
& \varrho_{c}\left(\mathbf{r}_{c}^{\prime}, \mathbf{r}_{c}, t\right)=\sum_{k} \lambda_{k}(t) \phi_{k}^{*}\left(\mathbf{r}_{c}^{\prime}, t\right) \phi_{k}\left(\mathbf{r}_{c}, t\right),
\end{aligned}
$$

i.e the formula (3.5) contains the eigenvectors $\phi_{k}, \psi_{k}$ as the Schmidt basis functions, and the countably many common eigenvalues $\lambda_{k}(t)$ of $\varrho_{e}$ and $\varrho_{c}$ density matrices respectively. We note that in this continuous variable case the diagonalization (3.6) or (3.7) actually involves the solution of a homogenous Fredholm integral equation of the second kind. In addition - contrary to discrete variable systems - these density matrices are usually highly singular, due to the trace condition $\operatorname{Tr} \varrho_{e}=\operatorname{Tr} \varrho_{c}=1$ they contain infinitely many zero or close to zero eigenvalues. Therefore, it is also necessary to introduce an ordering of the eigenvalues $\lambda_{1} \geq \lambda_{2} \geq \lambda_{3} \geq \ldots$ and then to use only a finite number of them which are greater than an adequately small threshold number $\varepsilon$.

\subsubsection{Entanglement entropies}

The eigenvalues $\lambda_{k}(t)$ allow one to quantify the entanglement of the particles (subsystems) $e$ and $c$ by introducing quantum entropies $[98,99]$. Most frequently we use in this thesis the von Neumann entropy

$$
S_{N}(t)=-\operatorname{Tr}\left[\hat{\varrho}_{c}(t) \ln \hat{\varrho}_{c}(t)\right]=-\sum_{k} \lambda_{k}(t) \ln \lambda_{k}(t),
$$


and in certain cases the linear entropy

$$
S_{L}(t)=1-\operatorname{Tr}\left[\hat{\varrho}_{c}^{2}(t)\right]=1-\sum_{k} \lambda_{k}^{2}(t)
$$

The von Neumann entropy obeys some natural requirements, and it also has a quantum information theoretic appeal [100] while the linear entropy (3.9) is easier to calculate, since diagonalization is not necessary. However, both of these entropies generally tend to behave the same way in this simple bipartite configuration: if a subsystem is in a pure state they assume the value 0 , and they increase as the "mixedness" of the subsystem's state increases. It is important that this quantification does not straightforwardly generalize to the case where the composite system is divided into more than two subsystems [101].

For independent systems the total density operator is the tensor product of those of the subsystems and then the Neumann entropy of the composite system is exactly the sum of the Neumann entropies of the subsystems. In our case, however, when by the very nature of the problem $e$ and $c$ are not independent, only strong subadditivity holds [102], which gives an upper bound of the composite system's entropy as

$$
S_{N}\left(\varrho_{e c}\right) \leq S_{N}\left(\varrho_{e}\right)+S_{N}\left(\varrho_{c}\right)
$$

A useful lower bound is given by the Araki-Lieb inequality as

$$
\left|S_{N}\left(\varrho_{e}\right)-S_{N}\left(\varrho_{c}\right)\right| \leq S_{N}\left(\varrho_{e c}\right)
$$

These two properties can be used to form analytical criteria that approximate entanglement quantizations should meet.

\subsection{Elements of quantum information theory}

In this section, we quantify the quantum correlation information between two arbitrary systems using elements of quantum informational theory. In this general case, the composite bipartite system $\varrho_{e c}$ can be in a mixed state, for example, in the directionally reduced $z_{e}-z_{c}$ bipartite systems or in a thermodynamic equilibrium. The entangled pure state density matrix $\varrho_{e c}^{(\text {pure })}$ defined in (3.2) is important special case of $\varrho_{e c}$.

\subsubsection{Correlation types and quantum information}

Quantum information theory, in parallel to the classical Shannon information theory [103], succinctly associates information with randomness shared by two physical ensembles, but is based entirely on density matrices (rather than probability distributions) for the description of quantum ensembles. Its formalism also allows for a consistent description of the characteristics of the correlation channel in a bipartite system $e c$. The central object of quantum information theory is the entropy (3.8) introduced by Neumann.

In general, $\varrho_{e c}$ could involve both classical and quantum correlations. Then it is crucial to 
recognize the features of these, thus we recall their meaning first. If a bipartite system contains only classical correlations between the two subsystems, then it has a density matrix of the following form:

$$
\varrho_{e c}^{(\mathrm{cl})}=\sum_{k} w_{k} \cdot \varrho_{e}^{(k)} \otimes \varrho_{c}^{(k)}
$$

where $w_{k}$ satisfy $\sum_{k} w_{k}=1$ and $w_{k} \geq 0$. We are dealing with some form of quantum entanglement only if the density matrix of the system does not satisfy (3.12). We denote the corresponding class of nonclassical density matrices generally as $\varrho_{e c}^{\text {(quant) }}$.

In the following, we recall the properties of the quantum conditional and quantum mutual entropies of quantum information theory that suit the task of determination and quantification of entanglement. We will denote the composite system by $E C$, and its subsystems by $E$ and $C$. We also simplify the notation of the entropies as $S(E C)=S_{N}\left(\varrho_{e c}\right), S(E)=S_{N}\left(\varrho_{e}\right), S(C)=S_{N}\left(\varrho_{c}\right)$ where $S_{N}$ is the Neumann entropy (3.8).

\subsubsection{Quantum conditional entropy}

The quantum conditional entropy corresponding to a subsystem can be introduced based on the conditional density or amplitude operator $[104,105]$, but we consider the following formula for the definition

$$
S(C \mid E)=S(E C)-S(E)
$$

for the quantum conditional entropy of subsystem $C$, and $S(E \mid C)$ is the quantum conditional entropy of subsystem $E$. This characterizes the remaining entropy or information of $C$ after $E$ has been measured completely. We illustrate the quantum entropies of $E C$ in Fig. 3.1 (a) including the conditional entropies.

We can interpret both of these quantum conditional entropies generally the same way as the classical ones, but they can have negative values. They behave exactly the same way for classical correlations as their classical counterparts: they are nonnegative

$$
\varrho_{e c}^{(\mathrm{cl})} \Longrightarrow S(C \mid E) \geq 0 \text { and } S(E \mid C) \geq 0 \text {. }
$$

However, when either of them is negative,

$$
S(C \mid E) \leq 0 \text { or } S(E \mid C) \leq 0 \Longrightarrow \varrho_{e c}^{(\text {quant })}
$$

then the composite system is entangled, which leads e.g. to a violation of the Bell inequalities. Note that the converses of (3.14) and (3.15) are not true and also $S(E)-S(E C)$ can be positive in case of quantum entanglement. For example, in case of pure composite systems we have

$$
\varrho_{e c}^{(\text {pure })} \Longrightarrow S(C \mid E)=-S(E)=-S(C)
$$

and $S(C)=S(E)$ is positive. Because of this, quantum entanglement is sometimes called "supercorrelation" and introduces virtual information which describes that the measurement changes the quantum state of the other subsystem. 
(a)

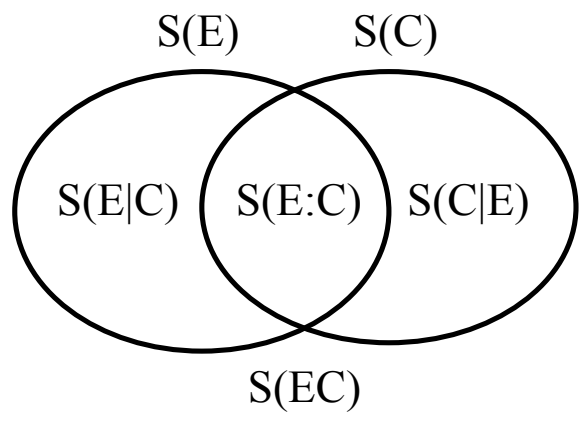

(b)

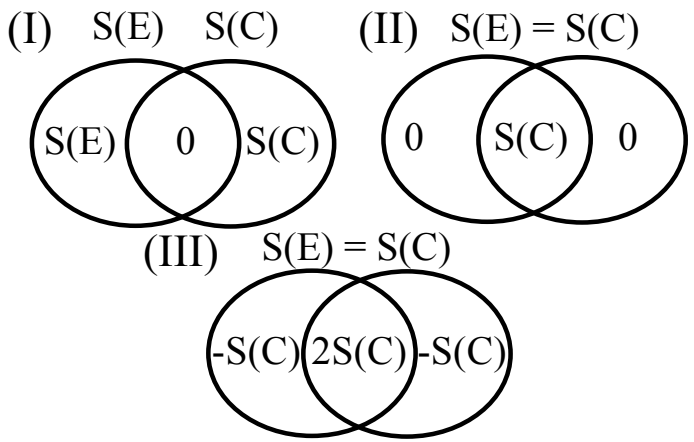

Figure 3.1: (a) General entropy diagram for a quantum bipartite system $E C$. (b) Entropy diagrams of special cases: (I) independent, (II) a classically correlated with $S(E C)=S(E: C)$, (III) purely quantum entangled subsystems. We can see in (III) that quantum entanglement introduce virtual (negative) information.

\subsubsection{Quantum mutual entropy}

Quantum mutual entropy is the shared entropy or shared information between subsystems $E$ and $C$. It can be defined using the mutual density or amplitude operator [104], but we use the definition

$$
S(E: C)=S(E)+S(C)-S(E C) .
$$

It can be also interpreted as the decrease of entropy of subsystem $C$ due to the knowledge of $E$ (and vice-versa). Because of this, we note that the conditional entropy and mutual entropy are related in the respective subsystems as

$$
S(E: C)=S(C)-S(C \mid E)
$$

We also illustrate this entropy in Fig. 3.1 (a).

The quantum mutual entropy is by construction symmetric and its values are always nonnegative. For classical correlations the following relation holds:

$$
\varrho_{e c}^{(\mathrm{cl})} \Longrightarrow S(E: C) \leq \min [S(E), S(C)] \text {. }
$$

If the values of $S(E: C)$ extend above this classical limit then there is quantum entanglement between $E$ and $C$ :

$$
\min [S(E), S(C)] \leq S(E: C) \Longrightarrow \varrho_{e c}^{\text {(quant) }} .
$$

Unfortunately again, it is not true that below the classical limit (3.19) there could not be quantum effects between the two subsystems. The upper limit of the quantum mutual entropy is

$$
S(E: C) \leq 2 \min [S(E), S(C)]
$$

which can be derived from the Araki-Lieb inequality (3.11).

It is instructive to observe that for pure state composite systems, like EPR pairs, $S(E: C)$ is at the upper limit:

$$
\varrho_{e c}^{(\text {pure })} \Longrightarrow S(E: C)=S(E)+S(C)=2 S(C) .
$$


We illustrate entropy diagrams for a few special systems in Fig. 3.1 (b) including the a special classically correlated and purely quantum entangled cases. These are important in the sense that they are at the upper bound in (3.19) and (3.20) respectively.

Based on this and using the exactness of (3.22), a unified entanglement or quantum nonseparability measure can be defined which we denote as the average mutual entropy:

$$
\bar{S}(E: C)=\frac{1}{2} S(E: C)
$$

which is the same as (3.8) in pure bipartite quantum systems. We can also use this to deduce whether we are dealing with entanglement: if we are near the limit (3.21), i.e. $\bar{S}$ is close to $\min [S(E), S(C)]$, then entanglement is the major correlation. The formulas (3.17), (3.13), (3.23) can be used for the analysis of the entanglement dynamics of the directional bipartite subsystems of (3.2). But we have to be careful because (3.23) is a general measure of correlations and entanglement e.g. nonseparability, and does not imply entanglement under general conditions. 


\section{Part II}

New results 


\section{Chapter 4}

\section{The hybrid splitting algorithm}

In the present chapter, we propose a novel method to accurately incorporate the effect of the Coulomb singularity into the solution of a spinless, axially symmetric TDSE in cylindrical coordinates. We account for the singular Coulomb potential through an analytic boundary condition which resolves the problem of nondifferentiability of the hydrogen eigenfunctions in the cylindrical coordinate system and yields high-order spatial accuracy. We also discuss the case of different $m$ quantum numbers. We published this method in [P1].

In Section 4.2, we introduce our hybrid splitting method, which retains the robustness of the full Crank-Nicolson method (Section 2.1.2) and the speed of the directional split-operator method (Section 2.2.2). Then we analyze the newly arisen coefficient matrix, we describe the way how to take advantage of its structure, then we derive a special solver algorithm which is necessary for the fast solution of the TDSE. In Section 4.3, we discuss the results of numerical experiments we carried out with our hybrid splitting method: (i) for the accuracy of the spatial discretization of the finite difference scheme, (ii) for the temporal accuracy of our split-step finite difference scheme combined with high-order evolution operator factorizations, and (iii) for final verification, we simulate the hydrogen atom in a time-dependent external electric field.

\subsection{The Schrödinger equation and its boundary conditions}

Our aim is to represent the singularity of the 3D Coulomb potential by implementing the correct boundary condition at the origin. As other kind of numerical errors decrease, the artifacts caused by the smoothing of the singularity become more disturbing. Therefore, the derivation of this boundary condition is needed for the numerical method to handle it as accurately and effectively as possible.

\subsubsection{The boundary condition for the 3D singular Coulomb potential}

In order to find this condition, it seems necessary to use spherical polar coordinates, since the Coulomb problem is not separable in cylindrical coordinates. Let us first discuss the properties 
of the radial solutions $\phi(r)$ of the well-known radial equation $[59,106]$ :

$$
\left[\beta \frac{\partial^{2}}{\partial r^{2}}+\frac{2 \beta}{r} \frac{\partial}{\partial r}+\beta \frac{l(l+1)}{r^{2}}-\frac{Z}{r}\right] \phi(r)=E \phi(r)
$$

where $l$ is the angular momentum quantum number and $\beta=-1 / 2 \mu$. If we multiply both sides with $r$ then take the $r \rightarrow 0$ limit, we can acquire Robin boundary condition for all of the $l=0$ states as

$$
\left.\frac{\partial \phi}{\partial r}\right|_{r=0}=-\mu Z \phi(0)
$$

but the bound states with higher angular momenta will have $\phi(0)=0$ boundary condition at the origin, in accordance with the fact that for $l>0$ the particle physically cannot penetrate to $r=0$, because of the singularity of $l(l+1) / r^{2}$.

Since we need the boundary conditions in cylindrical coordinates independent of the $l$ values, we turn to the nonseparated symmetric spherical Schrödinger equation for answers:

$$
\left[\beta \frac{\partial^{2}}{\partial r^{2}}+\frac{2 \beta}{r} \frac{\partial}{\partial r}+\beta \frac{1}{r^{2} \sin \theta} \frac{\partial}{\partial \theta}\left(\sin \theta \frac{\partial}{\partial \theta}\right)-\frac{Z}{r}\right] \Psi(r, \theta)=E \Psi(r, \theta) .
$$

which has also the term $1 / r^{2}$ and has singularity at $r=0$ and at $\theta=k \pi, k=0,1,2 \ldots$ To acquire the boundary conditions for $r=0$, we again multiply both sides with $r$ and take the $r \rightarrow 0$ limit, yielding

$$
\lim _{r \rightarrow 0}\left[2 \beta \frac{\partial}{\partial r}+\beta \frac{\cos \theta}{r \sin \theta} \frac{\partial}{\partial \theta}+\frac{\beta}{r} \frac{\partial^{2}}{\partial^{2} \theta}-Z\right] \Psi(r, \theta)=0 .
$$

Now we transform equation (4.4) into cylindrical coordinates $z=r \cos \theta$ and $\rho=r \sin \theta$, using following expressions of the partial derivatives:

$$
\begin{gathered}
\frac{\partial}{\partial r}=\frac{\partial z}{\partial r} \frac{\partial}{\partial z}+\frac{\partial \rho}{\partial r} \frac{\partial}{\partial \rho}=\sin \theta \frac{\partial}{\partial \rho}+\cos \theta \frac{\partial}{\partial z} \\
\frac{\partial}{\partial \theta}=\frac{\partial z}{\partial \theta} \frac{\partial}{\partial z}+\frac{\partial \rho}{\partial \theta} \frac{\partial}{\partial \rho}=r \cos \theta \frac{\partial}{\partial \rho}-r \sin \theta \frac{\partial}{\partial z}
\end{gathered}
$$

with $\cos \theta=z / \sqrt{z^{2}+\rho^{2}}$ and $\sin \theta=\rho / \sqrt{z^{2}+\rho^{2}}$. After writing back and taking the limit, the formula (4.4) becomes

$$
\left.\left[2 \beta \frac{\rho}{\sqrt{z^{2}+\rho^{2}}} \frac{\partial}{\partial \rho}+\beta \frac{z}{\sqrt{z^{2}+\rho^{2}}} \frac{\partial}{\partial z}+\beta \frac{z^{2}}{\rho \sqrt{z^{2}+\rho^{2}}} \frac{\partial}{\partial \rho}-Z\right] \Psi(r, \theta)\right|_{r=0}=0
$$

Then, by substituting $z=0$ into (4.7), we obtain the Robin boundary condition for the 3D Coulomb singularity:

$$
\left.\frac{\partial \Psi}{\partial \rho}\right|_{\rho, z=0}=-\mu Z \Psi(0,0, t) .
$$

This can be generalized to include multiple Coulomb-cores rather easily, we just need to impose 
(4.8) at multiple $z, \rho$ points along the $\rho=0$ axis. It is interesting to note also that the Robin boundary condition imposed by a 1D Dirac-delta potential (1.16) also has the form of (4.8) for a symmetric wave function [59]. Additionally, any continuously differentiable potential added to this configuration does not change this boundary condition.

\subsubsection{The states with nonzero magnetic quantum numbers}

Although we are mainly interested in the solution of the TDSE with an axially symmetric initial state, i.e. an initial state of zero magnetic quantum number $m$, we briefly show in the following that initial states with nonzero $m$ also lead to a TDSE of the form (1.6), however with different boundary conditions at $\rho=0$.

Let us write out the full 3D time-dependent Schrödinger equation in cylindrical coordinates with the same axially symmetric potential that we used previously:

$$
i \frac{\partial}{\partial t} \Xi(z, \rho, \varphi, t)=\left[\beta \frac{\partial^{2}}{\partial z^{2}}+\beta \frac{\partial^{2}}{\partial \rho^{2}}+\frac{\beta}{\rho} \frac{\partial}{\partial \rho}+\frac{\beta}{\rho^{2}} \frac{\partial^{2}}{\partial \phi^{2}}+V(z, \rho, t)\right] \Xi(z, \rho, \varphi, t) .
$$

We can write the quantum state with a given magnetic quantum number $m$ in the following form for any $t$

$$
\Xi(z, \rho, \varphi, t)=\Psi_{m}(z, \rho, t) e^{i m \varphi},
$$

due to the fact that the Hamiltonian of (4.9) conserves the $m$ quantum number. (We can also use real angular basis functions like $\sin (m \varphi)$ or $\cos (m \varphi)$ instead of $e^{i m \varphi}$, as long as that they are the eigenfunctions of $\partial_{\varphi}^{2}$.)

After substituting (4.10) to (4.9) we perform projection in the form of $\int_{0}^{2 \pi} e^{-i m \varphi}(\cdot) \mathrm{d} \varphi$. Then we get the TDSE for our wave function $\Psi_{m}(z, \rho, t)$ with the specified magnetic quantum number $m$ :

$$
i \frac{\partial}{\partial t} \Psi_{m}(z, \rho, t)=\left[\beta \frac{\partial^{2}}{\partial z^{2}}+\beta \frac{\partial^{2}}{\partial \rho^{2}}+\frac{\beta}{\rho} \frac{\partial}{\partial \rho}-\beta \frac{m^{2}}{\rho^{2}}+V(z, \rho, t)\right] \Psi_{m}(z, \rho, t),
$$

Eq. (4.11) has the same form as the axially symmetric equation (1.6) but with a new $m$ dependent potential:

$$
V_{m}(z, \rho, t)=V(z, \rho, t)-\beta m^{2} / \rho^{2} .
$$

However, if $m \neq 0$ then the $1 / \rho^{2}$ singularity of (4.12) alters the boundary condition (1.10) at the $\rho=0$ axis to the following:

$$
\Psi_{m}(z, 0, t)=0,
$$

which can by derived by multiplying (4.11) with $\rho^{2}$ and taking the limit of $\rho \rightarrow 0$. The Neumann boundary condition (4.13) also works with Coulomb potential, and in every case where $\rho^{2} V(z, \rho, t)=0$ in the limit of $\rho \rightarrow 0$. It is also consistent with the boundary conditions for Coulomb states with nonzero angular momenta $(l \neq 0)$ mentioned in the previous section. 


\subsection{Hybrid splitting algorithm}

Previously, in Section 2.1, we derived the suitable finite-difference scheme with high-order spatial accuracy, using second-order Crank-Nicolson method and we saw that it is capable of handling the boundary conditions at $\rho=0$ properly. We also discussed the standard splitoperator methods for numerically solving the TDSE and we introduced the concept of splitstep finite difference methods. In that case, the approximate operations count of evaluating the formula (2.38) scales linearly with the number of spatial gridpoints $N_{z} N_{\rho}$, but the method suffers from error blow up near the atomic core. Now, we propose a merger of this split-operator method with the cylindrical 2D Crank-Nicolson scheme in the form of "hybrid splitting" to get the best of both worlds.

\subsubsection{Hybrid splitting of the exponential operator}

The key concept of our method is that we split the Hamiltonian both according to directions and spatial domains in order to remove the problematic behavior of the split-operator method at the $\rho=0$ edge.

\section{Symmetric splitting scheme}

First, we split the spatial domain of the simulations as $G=G_{\mathrm{CN}}+G_{\text {Split }}$ where

$$
\begin{gathered}
G=\left\{z, \rho \in \mathbb{R}, z_{\min } \leq z \leq z_{\max }, 0 \leq \rho \leq \rho_{\max }\right\}, \\
G_{\mathrm{CN}}=\left\{z, \rho \in \mathbb{R}, z_{\min } \leq z \leq z_{\max }, 0 \leq \rho \leq L \Delta \rho\right\}, \\
G_{\text {Split }}=\left\{z, \rho \in \mathbb{R}, z_{\min } \leq z \leq z_{\max }, L \Delta \rho<\rho \leq \rho_{\max }\right\},
\end{gathered}
$$

then we define the pieces of the Hamiltonian $H=H_{z}+H_{\rho}+H_{\mathrm{CN}}$ as

$$
\begin{gathered}
H_{z}=\beta \partial_{z}^{2} \quad \text { if }(z, \rho) \in G_{\text {Split }} \\
H_{\rho}=\beta \partial_{\rho}^{2}+\beta \rho^{-1} \partial_{\rho}+V(z, \rho, t) \quad \text { if }(z, \rho) \in G_{\text {Split }}, \\
H_{\mathrm{CN}}=\beta \partial_{z}^{2}+\beta \partial_{\rho}^{2}+\beta \rho^{-1} \partial_{\rho}+V(z, \rho, t) \quad \text { if }(z, \rho) \in G_{\mathrm{CN}},
\end{gathered}
$$

where $\beta=-1 / 2 \mu$. Then the original Hamiltonian can be reconstructed as

$$
H= \begin{cases}H_{z}+H_{\rho} & \text { if }(z, \rho) \in G_{\mathrm{Split}} \\ H_{\mathrm{CN}} & \text { if }(z, \rho) \in G_{\mathrm{CN}}\end{cases}
$$

$H$ will never get evaluated outside $G$, in accordance with the boundary conditions for the wave function $\Psi(z, \rho, t)$.

Thus, we have partitioned the spatial domain into two regions: $G_{\mathrm{CN}}$ where (based on the previous section) we do not use any split-operator approximation and propagate with Hamiltonian $H_{\mathrm{CN}}$, and region $G_{\text {Split }}$ where we do use operator splitting as $H_{z}+H_{\rho}$. 

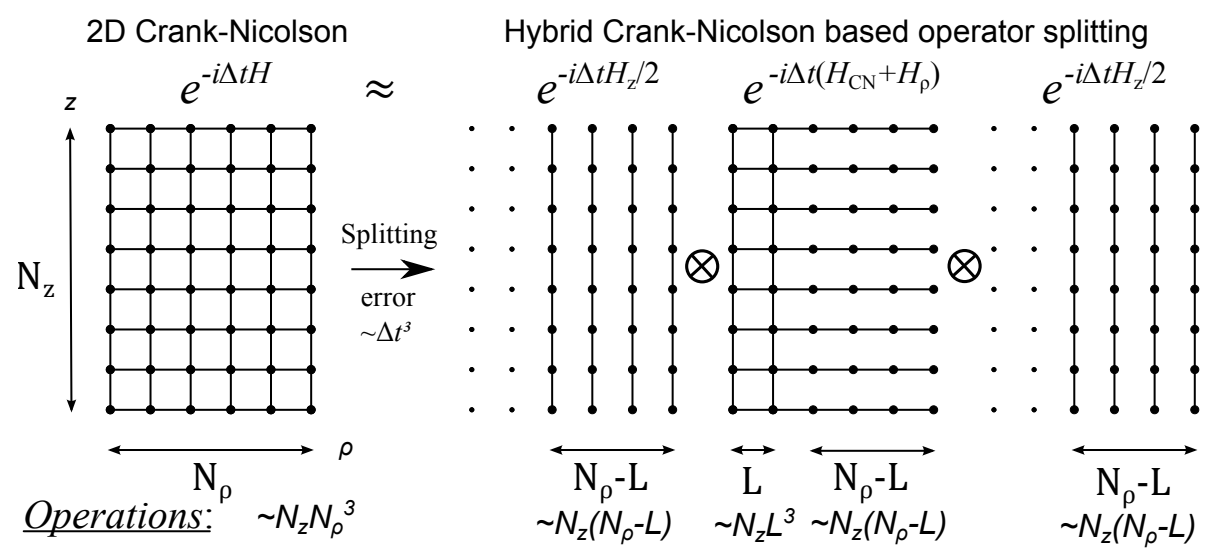

Figure 4.1: Sketch of our hybrid splitting scheme. The costly 2D Crank-Nicolson scheme was replaced with a special second-order symmetric split-operator formula except at the $L$ nearest gridpoints in the neighborhood of the $\rho=0$ axis, in order to retain accuracy and stability. The solid lines represent coupling between the gridpoints of the exponential operator evaluations. We also indicate the approximate operations count needed to solve the respective systems of linear equations.

In order to merge the directional operator splitting approach with the true 2D Crank-Nicolson method, we introduce our second-order hybrid splitting scheme:

$$
e^{-i \Delta t\left(H_{z}+H_{\rho}+H_{\mathrm{CN}}\right)}=e^{-i \Delta t H_{z} / 2} e^{-i \Delta t\left(H_{\rho}+H_{\mathrm{CN}}\right)} e^{-i \Delta t H_{z} / 2}+O\left(\Delta t^{3}\right),
$$

where we keep $H_{\mathrm{CN}}$ and $H_{\rho}$ in the same exponent, in order that the wave function could "freely flow" between the two regions $G_{\mathrm{CN}}$ and $G_{\text {Split }}$ without introducing further artifacts. (Note that the exponential operator $e^{-i \Delta t\left(H_{\rho}+H_{\mathrm{CN}}\right)}$ cannot be split further in this sense.) We use this scheme as the second-order terms in the iterative formulas (2.31), (2.35), (2.37) to gain higher-order accuracy in $\Delta t$. The $e^{-i \Delta t H_{z} / 2}$ part can be evaluated with any method of choice. We have constructed a special Numerov-extended Crank-Nicolson line propagation algorithm for this, which can be found in Appendix B. We illustrate the hybrid splitting formula (4.21) in Fig. 4.1.

\section{The Crank-Nicolson form of the central exponential}

In order to numerically evaluate the $e^{-i \Delta t\left(H_{\rho}+H_{\mathrm{CN}}\right)}$ operator, we apply the second-order Padéapproximation (2.10) to arrive at a second-order Crank-Nicolson form of

$$
\Psi\left(t_{k+1}\right)=e^{-i \Delta t\left(H_{\rho}+H_{\mathrm{CN}}\right)} \Psi\left(t_{k}\right)=\left(1+\alpha\left(H_{\rho}+H_{\mathrm{CN}}\right)\right)^{-1}\left(1-\alpha\left(H_{\rho}+H_{\mathrm{CN}}\right)\right) \Psi\left(t_{k}\right)+O\left(\Delta t^{3}\right)
$$

where $\alpha=i \Delta t / 2$ and $V(z, \rho, t)$ is evaluated at the midpoint $t+\Delta t / 2$. We introduce the spatial grids (2.12), (2.13) and the discrete operators $\widetilde{L}_{z}, \widetilde{L}_{\rho}$ and $\widetilde{D}_{\rho}$, then we get the following equations for the two domains for all $i \in\left[0, N_{z}\right]$ :

$$
\begin{gathered}
\left(1+\alpha \beta \widetilde{L}_{\rho}+\alpha \beta \widetilde{L}_{z}+\alpha V_{i, j}\right) \Psi_{i, j}\left(t_{k+1}\right)=\left(1-\alpha \beta \widetilde{L}_{\rho}-\alpha \beta \widetilde{L}_{z}-\alpha V_{i, j}\right) \Psi_{i, j}\left(t_{k}\right), \text { if } j \in[1, L] \\
\left(1+\alpha \beta \widetilde{L}_{\rho}+V_{i, j}\right) \Psi_{i, j}\left(t_{k+1}\right)=\left(1-\alpha \beta \widetilde{L}_{\rho}-\alpha V_{i, j}\right) \Psi_{i, j}\left(t_{k}\right), \text { if } j \in\left[L+1, N_{\rho}\right]
\end{gathered}
$$


Using five-point finite differences, the first set of equations (4.23) can be expanded resulting in the form of (2.19), and the expansion of the second set (4.24) gives the following result for all $i \in\left[0, N_{z}\right], j \in\left[L+1, N_{\rho}\right]$ :

$$
\begin{gathered}
(-1+1 / j) \beta_{\rho} X_{i, j-2}+(16-8 / j) \beta_{\rho} X_{i, j-1}+\left(1-30 \beta_{\rho}+\alpha V_{i, j}\right) X_{i, j} \\
+(16+8 / j) \beta_{\rho} X_{i, j+1}+(-1-1 / j) \beta_{\rho} X_{i, j+2}=(1-1 / j) \beta_{\rho} \Psi_{i, j-2}+(-16+8 / j) \beta_{\rho} \Psi_{i, j-1} \\
+\left(1+30 \beta_{\rho}-\alpha V_{i, j}\right) \Psi_{i, j}+(-16-8 / j) \beta_{\rho} \Psi_{i, j+1}+(1+1 / j) \beta_{\rho} \Psi_{i, j+2},
\end{gathered}
$$

where the symbols $\beta_{\rho}$ and $\beta_{z}$ are given in (2.20) with $X_{i, j}=\Psi_{i, j}\left(t_{k+1}\right)$ and $\Psi_{i, j}=\Psi_{i, j}\left(t_{k}\right)$.

Assuming that the potential is smooth, the Neumann boundary condition (2.18) prescribes the implicit equations at $\rho=0$. However, if a Coulomb-core of strength $Z$ is present at the origin and $m=0$, then according to (4.8), it will be replaced at $z=0$. We can write all Neumann and Robin boundary conditions at $\rho=0$ for the $m=0$ configuration for all $i \in\left[0, N_{z}\right]$ as the following:

$$
\left(\widetilde{D}_{\rho}+\mu Z \delta_{R, i}\right) \Psi_{i, j}\left(t_{k+1}\right)=0
$$

Here we have assumed that the origin is included in the $z_{i}$ grid (2.12) with $z_{R}=0$, where $R$ can be anywhere in the interval $\left[0, N_{z}\right]$ if it is reasonably far from the box boundaries. This has the expanded form of

$$
\left(-25+12(\mu Z) \Delta \rho \cdot \delta_{R, i}\right) X_{i, 0}+48 X_{i, 1}-36 X_{i, 2}+16 X_{i, 3}-3 X_{i, 4}=0
$$

For the $m \neq 0$ states, instead of (4.27), we just use the Dirichlet-boundary condition (4.13) as

$$
X_{i, 0}=0
$$

The box boundary conditions also apply independent of the value of the $m$ quantum number:

$$
X_{i, j}=\Psi_{i, j}=0, \text { if } i \notin\left[0, N_{z}\right], j \notin\left[-N_{\rho}, N_{\rho}\right] .
$$

From these, a mixed 2D - 1D Crank-Nicolson scheme can be constructed in the $G_{\mathrm{CN}}+G_{\text {Split }}$ domain depending on $m$, which is fourth-order accurate in space and second-order accurate in time.

What are the advantages of this scheme? First, if $L>1 / \Delta \rho$ the accuracy of the scheme increases considerably in the presence of the Coulomb potential, because we removed directional splitting near its core (where its gradient is the largest). Second, if $L \geq 5$ (the "width" of $\widetilde{D}_{\rho}$ ) then the (4.26) condition no longer affects into the split-operator zone: we can maintain robustness. Third, the operation-count is approximately $\sim L^{3} N_{z}$ plus $\sim\left(N_{\rho}-L\right) N_{z}$, meaning if $L \ll N_{\rho}$ then we can regain the speed of the directional splitting and large part of the algorithm, corresponding to equations (4.24) can be parallelized for different $j$ indices. So, if we set $L$ to the smallest value sufficient for accuracy then we can acquire a very efficient scheme. In order to solve resulting linear equations effectively, we present our special algorithm, which we call "hybrid splitting solver algorithm". 


\subsubsection{Hybrid splitting solver algorithm}

In this section, we write out the matrix form of the linear equations resulting from the approximation of the exponential operator $e^{-i \Delta t\left(H_{\rho}+H_{\mathrm{CN}}\right)}$ to familiarize ourselves with its structure, which is required for developing an efficient propagation algorithm for our hybrid splitting scheme. Then we outline the solution algorithm.

\section{The matrix form of the linear equations}

Let us construct the column vectors corresponding to the $i$ th row of the $2 \mathrm{D}$ problem as

$$
\Psi_{i}=\left(\begin{array}{lllll}
\Psi_{i, 0} & \Psi_{i, 1} & \Psi_{i, 2} & \ldots & \Psi_{i, N_{\rho}}
\end{array}\right)^{\mathrm{T}} \text { and } \mathbf{X}_{i}=\left(\begin{array}{lllll}
X_{i, 0} & X_{i, 1} & X_{i, 2} & \ldots & X_{i, N_{\rho}}
\end{array}\right)^{\mathrm{T}} .
$$

Then, the joint problem (4.23), (4.24) will take the block pentadiagonal form of

$$
\left[\begin{array}{ccccccc}
\mathrm{B}_{0} & \mathrm{C}_{0} & \mathrm{~F}_{0} & 0 & 0 & \ldots & 0 \\
\mathrm{~A}_{1} & \mathrm{~B}_{1} & \mathrm{C}_{1} & \mathrm{~F}_{1} & 0 & \ldots & 0 \\
\mathrm{E}_{2} & \mathrm{~A}_{2} & \mathrm{~B}_{2} & \mathrm{C}_{2} & \mathrm{~F}_{2} & \ldots & 0 \\
\vdots & \ddots & \ddots & \ddots & \ddots & \ddots & \vdots \\
0 & \ldots & \mathrm{E}_{N_{z}-2} & \mathrm{~A}_{N_{z}-2} & \mathrm{~B}_{N_{z}-2} & \mathrm{C}_{N_{z}-2} & \mathrm{~F}_{N_{z}-2} \\
0 & \ldots & 0 & \mathrm{E}_{N_{z}-1} & \mathrm{~A}_{N_{z}-1} & \mathrm{~B}_{N_{z}-1} & \mathrm{C}_{N_{z}-1} \\
0 & \cdots & 0 & 0 & \mathrm{E}_{N_{z}} & \mathrm{~A}_{N_{z}} & \mathrm{~B}_{N_{z}}
\end{array}\right]\left[\begin{array}{c}
\mathbf{X}_{0} \\
\mathbf{X}_{1} \\
\mathbf{X}_{2} \\
\vdots \\
\mathbf{X}_{N_{z}-2} \\
\mathbf{X}_{N_{z}-1} \\
\mathbf{X}_{N_{z}}
\end{array}\right]=\left[\begin{array}{c}
\mathbf{y}_{0} \\
\mathbf{y}_{1} \\
\mathbf{y}_{2} \\
\vdots \\
\mathbf{y}_{N_{z}-2} \\
\mathbf{y}_{N_{z}-1} \\
\mathbf{y}_{N_{z}}
\end{array}\right]
$$

Here $\mathrm{E}_{i}, \mathrm{~A}_{i}, \mathrm{~B}_{i}, \mathrm{C}_{\mathrm{i}}, \mathrm{F}_{\mathrm{i}}$ are $\left(N_{\rho}+1\right) \times\left(N_{\rho}+1\right)$ matrices. Particularly,

$$
\begin{aligned}
& \mathrm{E}_{\mathrm{i}}=\left\{\operatorname{diag}\left(0, e_{z, i, 1}, \ldots, e_{z, i, L}, 0, \ldots, 0\right) \quad \text { if } i \in\left[2, N_{z}\right],\right. \\
& \mathrm{C}_{\mathrm{i}}=\left\{\operatorname{diag}\left(0, c_{z, i, 1}, \ldots, c_{z, i, L}, 0, \ldots, 0\right) \quad \text { if } i \in\left[1, N_{z}\right],\right. \\
& \mathrm{A}_{\mathrm{i}}=\left\{\operatorname{diag}\left(0, a_{z, i, 1}, \ldots, a_{z, i, L}, 0, \ldots, 0\right) \quad \text { if } i \in\left[0, N_{z}-1\right],\right. \\
& \mathrm{F}_{\mathrm{i}}=\left\{\operatorname{diag}\left(0, f_{z, i, 1}, \ldots, f_{z, i, L}, 0, \ldots, 0\right) \quad \text { if } i \in\left[0, N_{z}-2\right],\right.
\end{aligned}
$$

are the diagonal matrices responsible for coupling the adjacent $\rho$-rows (with different values of coordinate $z$ ), and $\mathrm{B}_{i}$ is an almost pentadiagonal matrix in the form of

$$
\mathrm{B}_{i}=\left[\begin{array}{ccccccc}
d_{0, i} & d_{1, i} & d_{2, i} & d_{3, i} & d_{4, i} & \ldots & 0 \\
a_{i, 0} & b_{i, 1} & c_{i, 1} & f_{i, 1} & 0 & \ldots & 0 \\
e_{i, 2} & a_{i, 2} & b_{i, 2} & c_{i, 2} & f_{i, 2} & \ldots & 0 \\
\vdots & \ddots & \ddots & \ddots & \ddots & \ddots & \vdots \\
0 & \ldots & e_{i, N_{\rho}-2} & a_{i, N_{\rho}-2} & b_{i, N_{\rho}-2} & c_{i, N_{\rho}-2} & f_{i, N_{\rho}-2} \\
0 & \ldots & 0 & e_{i, N_{\rho}-1} & a_{i, N_{\rho}-1} & b_{i, N_{\rho}-1} & c_{i, N_{\rho}-1} \\
0 & \ldots & 0 & 0 & e_{i, N_{\rho}} & a_{i, N_{\rho}} & b_{i, N_{\rho}}
\end{array}\right] .
$$


In the above matrices we have already taken into account the box, the Neumann and Robin boundary conditions for the $m=0$ configuration. The $d_{0, i}, d_{1, i}, d_{2, i}, d_{3, i}, d_{4, i}$ coefficients are given by the expanded equation (4.27) as

$$
d_{0, i}=-25+12(\mu Z) \Delta \rho \cdot \delta_{R, i}, d_{1, i}=48, d_{2, i}=-36, d_{3, i}=16, d_{4, i}=-3
$$

for all $i \in\left[0, N_{z}\right]$. These coefficients take the diagonal form of $d_{j, i}=\delta_{j, 0}$ for the $m \neq 0$ states.

In the Crank-Nicolson region $(j \leq L)$, the coefficients are given by equation (2.19):

$$
\begin{gathered}
b_{i, j}=1-30 \beta_{\rho}-30 \beta_{z}+\alpha V_{i, j}, a_{z, i, j}=c_{z, i, j}=16 \beta_{z} e_{z, i, j}=f_{z, i, j}=-\beta_{z}, \\
e_{i, j}=(-1+1 / j) \beta_{\rho}, f_{i, j}=(-1-1 / j) \beta_{\rho}, \\
a_{i, j}=(16-8 / j) \beta_{\rho}, c_{i, j}=(16+8 / j) \beta_{\rho} .
\end{gathered}
$$

In the split region $(j \geq L+1)$, we inspect the equation (4.25), and note that only $b_{i, j}$ get modified as

$$
b_{i, j}=1-30 \beta_{\rho}+\alpha V_{i, j} \quad \text { if } i \in\left[L+1, N_{\rho}\right], j \in\left[0, N_{z}\right]
$$

and the $a_{i, j}, c_{i, j}, e_{i, j}, f_{i, j}$ coefficients are the same as in (4.39), (4.40).

The right hand sides are given by (2.19) and (4.25), which can be written in a somewhat simpler matrix form which also takes into account the boundary conditions:

$$
\mathbf{y}_{i}=2 \Psi_{i}-\mathrm{E}_{i} \Psi_{i-2}-\mathrm{A}_{i} \Psi_{i-1}-\mathrm{B}_{i} \Psi_{i}-\mathrm{C}_{i} \Psi_{i+1}-\mathrm{F}_{i} \Psi_{i+2} \text { with } y_{i, 0}=0
$$

\section{Reducing the number of the equations}

One can see that the directional splitting introduced $N_{\rho}-L$ zeros at the end of the diagonal of the matrices $\mathrm{E}_{i}, \mathrm{~A}_{i}, \mathrm{C}_{\mathrm{i}}, \mathrm{F}_{\mathrm{i}}$ which means that the corresponding $\rho$-lines are not directly coupled. Taking advantage of this we significantly increase the computational efficiency by eliminating the improper matrix elements to reduce the effective block size to $(L+1)^{2}$. We do this in the following.

We take the equations corresponding to the $i$ th block matrix row

$$
\left[\begin{array}{lllllll}
\ldots & \mathrm{E}_{i} & \mathrm{~A}_{i} & \mathrm{~B}_{i} & \mathrm{C}_{i} & \mathrm{~F}_{i} & \ldots
\end{array}\right] \cdot \mathbf{X}=\mathbf{y}_{i}
$$

and write out their coefficient matrix from rows $L-1$ to $N_{\rho}$ :

$$
\left[\begin{array}{ccccccccccc}
\ldots & e_{i, L-1} & a_{i, L-1} & b_{i, L-1} & c_{i, L-1} & f_{i, L-1} & 0 & 0 & \ldots & 0 & \ldots \\
\ldots & 0 & e_{i, L} & a_{i, L} & b_{i, L} & c_{i, L} & f_{i, L} & 0 & \ldots & 0 & \ldots \\
\ldots & 0 & 0 & e_{i, L+1} & a_{i, L+1} & b_{i, L+1} & c_{i, L+1} & f_{i, L+1} & \ldots & 0 & \ldots \\
\ldots & \vdots & \vdots & \vdots & \ddots & \ddots & \ddots & \ddots & \ddots & \vdots & \ldots \\
\ldots & 0 & 0 & 0 & \ldots & e_{i, N_{\rho}-2} & a_{i, N_{\rho}-2} & b_{i, N_{\rho}-2} & c_{i, N_{\rho}-2} & f_{i, N_{\rho}-2} & \ldots \\
\ldots & 0 & 0 & 0 & \ldots & 0 & e_{i, N_{\rho}-1} & a_{i, N_{\rho}-1} & b_{i, N_{\rho}-1} & c_{i, N_{\rho}-1} & \ldots \\
\ldots & 0 & 0 & 0 & \ldots & 0 & 0 & e_{i, N_{\rho}} & a_{i, N_{\rho}} & b_{i, N_{\rho}} & \ldots
\end{array}\right]
$$


Here we remind that rows $j \leq L$ have extra nonzero entries far away from the diagonal, cf. (4.32) - (4.35). These lines cannot be used during the row operations, but the rest of them, with $j>L$ can be used. To reduce Eq. (4.31) to a smaller block pentadiagonal problem, $f_{i, L-1}, c_{i, L}$, $f_{i, L}$ must be eliminated for all $i \in\left[0, N_{z}\right]$. Then, the solution in the 2D Crank-Nicolson region $G_{\mathrm{CN}}$ will no longer depend on the solution in the directionally split region $G_{\text {Split }}$. The structure of the $\mathrm{B}_{i}$ matrix makes it possible to use the following backward elimination process from the $N_{\rho}$ th equation on, for all $i \in\left[0, N_{z}\right]$ :

$$
\left[\begin{array}{ccccccccccc}
\ldots & e_{i, L-1} & a_{i, L-1} & \tilde{b}_{i, L-1} & \tilde{c}_{i, L-1} & 0 & \ldots & 0 & 0 & 0 & \ldots \\
\ldots & 0 & e_{i, L} & \tilde{a}_{i, L} & \tilde{b}_{i, L} & 0 & \ldots & 0 & 0 & 0 & \ldots \\
\ldots & 0 & 0 & e_{i, L+1} & \tilde{a}_{i, L+1} & \tilde{b}_{i, L+1} & \ldots & 0 & 0 & 0 & \ldots \\
\ldots & \vdots & \vdots & \vdots & \ddots & \ddots & \ddots & \ddots & \ddots & \vdots & \ldots \\
\ldots & 0 & 0 & 0 & \ldots & e_{i, N_{\rho}-2} & \tilde{a}_{i, N_{\rho}-2} & \tilde{b}_{i, N_{\rho}-2} & 0 & 0 & \ldots \\
\ldots & 0 & 0 & 0 & \ldots & 0 & e_{i, N_{\rho}-1} & \tilde{a}_{i, N_{\rho}-1} & \tilde{b}_{i, N_{\rho}-1} & 0 & \ldots \\
\ldots & 0 & 0 & 0 & \ldots & 0 & 0 & e_{i, N_{\rho}} & \tilde{a}_{i, N_{\rho}} & \tilde{b}_{i, N_{\rho}} & \ldots
\end{array}\right]
$$

with right hand side of

$$
\widetilde{\mathbf{y}}_{i}=\left(\begin{array}{lllllllll}
y_{i, 0} & \ldots & y_{i, L-2} & \tilde{y}_{i, L-1} & \tilde{y}_{i, L} & \tilde{y}_{i, L+1} & \ldots & \tilde{y}_{i, N_{\rho}-1} & \tilde{y}_{i, N_{\rho}}
\end{array}\right)^{\mathrm{T}}
$$

where

$$
\begin{gathered}
\tilde{c}_{i, j}= \begin{cases}c_{i, j} & \text { if } j=N_{\rho}-1, \\
c_{i, j}-\left(f_{i, j} / \tilde{b}_{i, j+2}\right) \tilde{a}_{i, j+2} & \text { if } j=N_{\rho}-2 \ldots L-1,\end{cases} \\
\tilde{a}_{i, j}= \begin{cases}a_{i, j} & \text { if } j=N_{\rho}, \\
a_{i, j}-\left(\tilde{c}_{i, j} / \tilde{b}_{i, j+1}\right) e_{i, j+1} & \text { if } j=N_{\rho}-1 \ldots L,\end{cases} \\
\tilde{b}_{i, j}= \begin{cases}b_{i, j} & \text { if } j=N_{\rho}, \\
b_{i, j}-\left(\tilde{c}_{i, j} / \tilde{b}_{i, j+1}\right) \tilde{a}_{i, j+1} & \text { if } j=N_{\rho}-1, \\
b_{i, j}-\left(\tilde{c}_{i, j} / \tilde{b}_{i, j+1}\right) \tilde{a}_{i, j+1}-\left(f_{i, j} / \tilde{b}_{i, j+2}\right) e_{i, j+2} & \text { if } j=N_{\rho}-2 \ldots L, \\
b_{i, j}-\left(f_{i, j} / \tilde{b}_{i, j+2}\right) e_{i, j+2} & \text { if } j=L-1,\end{cases} \\
\tilde{y}_{i, j}= \begin{cases}y_{i, j} & \text { if } j=N_{\rho}, \\
y_{i, j}-\left(\tilde{c}_{i, j} / \tilde{b}_{i, j+1}\right) \tilde{y}_{i, j+1} & \text { if } j=N_{\rho}-1, \\
y_{i, j}-\left(\tilde{c}_{i, j} / \tilde{b}_{i, j+1}\right) \tilde{y}_{i, j+1}-\left(f_{i, j} / \tilde{b}_{i, j+2}\right) \tilde{y}_{i, j+2} & \text { if } j=N_{\rho}-2 \ldots L, \\
y_{i, j}-\left(f_{i, j} / \tilde{b}_{i, j+2}\right) \tilde{y}_{i, j+2} & \text { if } j=L-1,\end{cases}
\end{gathered}
$$

and the rest of the values remain unchanged. During the process, $\tilde{c}_{i, j}$ needs to be calculated first, then $\tilde{a}_{i, j}, \tilde{b}_{i, j}, \tilde{y}_{i, j}$ can be evaluated. Additionally, the value of $\tilde{c}_{i, j}$ is needed only in one step, then it can be discarded.

After the reduced equations are solved (cf. the next Section), we can solve for all variables 
with forward substitution:

$$
X_{i, j}= \begin{cases}\text { solution of the reduced block five diagonal part } & \text { if } j \leq L, \\ \left(\tilde{y}_{i, j}-\tilde{a}_{i, j} X_{i, j-1}-e_{i, j} X_{i, j-2}\right) / \tilde{b}_{i, j} & \text { if } j=L+1 \ldots N_{\rho} .\end{cases}
$$

These formulas above can be obtained by disassembling existing pentadiagonal solvers (for example [107]), however, care must be taken handling the boundary values at the $j=L$ edge and in the forward substitution afterwards.

Because of the special structure of the coefficient matrix in (4.31), this process of backward elimination and forward substitution can be parallelized to $N_{z}+1$ independent threads. They are depending on a synchronization step though, which consists of the solution of the following block pentadiagonal part.

\section{The reduced system to solve}

After we performed the above elimination procedure for every block $\mathrm{B}_{i}$, we obtain a block pentadiagonal system just like (4.31) with a drastically reduced block size, in the following form:

$$
\left[\begin{array}{ccccccc}
\widetilde{\mathrm{B}}_{0} & \widetilde{\mathrm{C}}_{0} & \widetilde{\mathrm{F}}_{0} & 0 & 0 & \ldots & 0 \\
\widetilde{\mathrm{A}}_{1} & \widetilde{\mathrm{B}}_{1} & \widetilde{\mathrm{C}}_{1} & \widetilde{\mathrm{F}}_{1} & 0 & \ldots & 0 \\
\widetilde{\mathrm{E}}_{2} & \widetilde{\mathrm{A}}_{2} & \widetilde{\mathrm{B}}_{2} & \widetilde{\mathrm{C}}_{2} & \widetilde{\mathrm{F}}_{2} & \cdots & 0 \\
\vdots & \ddots & \ddots & \ddots & \ddots & \ddots & \vdots \\
0 & \cdots & \widetilde{\mathrm{E}}_{N_{z}-2} & \widetilde{\mathrm{A}}_{N_{z}-2} & \widetilde{\mathrm{B}}_{N_{z}-2} & \widetilde{\mathrm{C}}_{N_{z}-2} & \widetilde{\mathrm{F}}_{N_{z}-2} \\
0 & \cdots & 0 & \widetilde{\mathrm{E}}_{N_{z}-1} & \widetilde{\mathrm{A}}_{N_{z}-1} & \widetilde{\mathrm{B}}_{N_{z}-1} & \widetilde{\mathrm{C}}_{N_{z}-1} \\
0 & \cdots & 0 & 0 & \widetilde{\mathrm{E}}_{N_{z}} & \widetilde{\mathrm{A}}_{N_{z}} & \widetilde{\mathrm{B}}_{N_{z}}
\end{array}\right]\left[\begin{array}{c}
\widetilde{\mathbf{X}}_{0} \\
\widetilde{\mathbf{X}}_{1} \\
\widetilde{\mathbf{X}}_{2} \\
\vdots \\
\widetilde{\mathbf{X}}_{N_{z}-2} \\
\widetilde{\mathbf{X}}_{N_{z}-1} \\
\widetilde{\mathbf{X}}_{N_{z}}
\end{array}\right]=\left[\begin{array}{c}
\widetilde{\mathbf{y}}_{0} \\
\widetilde{\mathbf{y}}_{1} \\
\widetilde{\mathbf{y}}_{2} \\
\vdots \\
\widetilde{\mathbf{y}}_{N_{z}-2} \\
\widetilde{\mathbf{y}}_{N_{z}-1} \\
\widetilde{\mathbf{y}}_{N_{z}}
\end{array}\right]
$$

These block matrices read as:

$$
\begin{aligned}
& \widetilde{\mathrm{E}}_{\mathrm{i}}=\left\{\operatorname{diag}\left(0, e_{z, i, 1}, \ldots, e_{z, i, L}\right) \quad \text { if } i \in\left[2, N_{z}\right],\right. \\
& \widetilde{\mathrm{C}}_{\mathrm{i}}=\left\{\operatorname{diag}\left(0, c_{z, i, 1}, \ldots, c_{z, i, L}\right) \quad \text { if } i \in\left[1, N_{z}\right],\right. \\
& \widetilde{\mathrm{A}}_{\mathrm{i}}=\left\{\operatorname{diag}\left(0, a_{z, i, 1}, \ldots, a_{z, i, L}\right) \quad \text { if } i \in\left[0, N_{z}-1\right]\right. \text {, } \\
& \widetilde{\mathrm{F}}_{\mathrm{i}}=\left\{\operatorname{diag}\left(0, f_{z, i, 1}, \ldots, f_{z, i, L}\right) \quad \text { if } i \in\left[0, N_{z}-2\right]\right. \text {, } \\
& \widetilde{\mathrm{B}}_{i}=\left[\begin{array}{ccccccc}
d_{0, i} & d_{1, i} & d_{2, i} & d_{3, i} & d_{4, i} & \ldots & 0 \\
a_{i, 0} & b_{i, 1} & c_{i, 1} & f_{i, 1} & 0 & \ldots & 0 \\
e_{i, 2} & a_{i, 2} & b_{i, 2} & c_{i, 2} & f_{i, 2} & \ldots & 0 \\
\vdots & \ddots & \ddots & \ddots & \ddots & \ddots & \vdots \\
0 & \cdots & e_{i, L-2} & a_{i, L-2} & b_{i, L-2} & c_{i, L-2} & f_{i, L-2} \\
0 & \cdots & 0 & e_{i, L-1} & a_{i, L-1} & b_{i, L-1} & \tilde{c}_{i, L-1} \\
0 & \cdots & 0 & 0 & e_{i, L} & \tilde{a}_{i, L} & \tilde{b}_{i, L}
\end{array}\right],
\end{aligned}
$$




$$
\begin{aligned}
\widetilde{\mathbf{y}}_{i} & =\left(\begin{array}{llllll}
y_{i, 0} & y_{i, 1} & \ldots & y_{i, L-2} & \tilde{y}_{i, L-1} & \tilde{y}_{i, L}
\end{array}\right)^{\mathrm{T}}, \\
\widetilde{\mathbf{X}}_{i} & =\left(\begin{array}{llllll}
X_{i, 0} & X_{i, 2} & \ldots & X_{i, L-2} & X_{i, L-1} & X_{i, L}
\end{array}\right)^{\mathrm{T}} .
\end{aligned}
$$

The method of acquiring the solution to this system can be chosen freely. For example, it can be solved by applying Gaussian elimination (with forward substitution) to the $(L+1)^{2}$ matrix blocks or directly to the $2 L+1$ wide diagonal matrix. In either case, the time to obtain the solution is drastically reduced (if $L \ll N_{\rho}$ ). After acquiring the solution of (4.52) we complete the hybrid splitting solver algorithm by formula (4.51), as indicated in the previous section.

\subsection{Results and discussion}

Now let us turn our attention to the numerical test results of the hybrid splitting method. First, in Section 4.3.1, we investigate the errors related to the spatial discretization. Section 4.3.2 is devoted to the tests of the time propagation errors. In Section 4.3.3 we demonstrate the performance of our algorithm through a real world example.

\subsubsection{Spatial accuracy: stationary state energies}

To investigate the errors related to the spatial discretization, we numerically compute the stationary states of selected test potentials. We calculate energy errors caused by the finite differences by comparing the numerical and the exact energy eigenvalues for certain energy eigenstates. We have also set $\Delta \rho=\Delta z$.

In these tests we have used our implementation of the true singular Coulomb potential (C). Another test case, called "Soft-Coulomb" potential (SC), differs from C only in that the boundary condition (4.26) at the origin is replaced by (2.18) at the origin. The results of both the C and the $\mathrm{SC}$ test cases are compared to the exact eigenenergies and eigenstates of $1 s, 2 s, 2 p_{z}$. We also compute the eigenenergy of the state $2 p_{x}$, where $\mathrm{C}$ and SC are identical (see Section 4.1.2). The energy errors of the 3D quantum harmonic oscillator $(\mathrm{H})$ are also tested for the ground and a first exited state. The most important features of our test potentials are summarized in Table 4.1.

We computed the eigenenergy values using imaginary time propagation method with our second-order hybrid splitting scheme using 4th-order accurate split-operators of form (2.37), where the parameter $\Delta t$ was chosen suitably small to minimize the spatial errors related to the operator splitting. These energy values were also verified with real time propagation, by comparing the time dependence of the phase factor of the eigenstates with the exact time dependence of $\Psi_{n}(z, \rho, t)=\psi_{n}(z, \rho) e^{-i E_{n} t}$ : the first two significant digits of the energy error were equal. It is worth noting that, although we used our hybrid splitting for calculations, these energy errors are related only to the spatial finite differences (that is, the 2D Crank-Nicolson scheme). For these results, it was sufficient to set the "Crank-Nicolson width" $L$ just above $\sim 1 / \Delta z$, further increase of $L$ did not improve the results.

From Fig. 4.2 and Table 4.2 we can draw several conclusions. In the case of the 3D harmonic oscillator (a reasonably smooth potential), the method is fourth-order accurate in $\Delta z$, as expected 
Target stationary states

\begin{tabular}{ccccccc}
\hline ID & Potential & Quantum numbers & Energy & State & Normalization & Parameters \\
\hline $\mathrm{C}-1 \mathrm{~s}$ & $-Z / r$ & $n=1, l=0, m=0$ & $-Z^{2} \mu / 2$ & $e^{-\mu Z r}$ & $\pi^{-1 / 2}(\mu Z)^{3 / 2}$ & $\mu=1, Z=1$ \\
$\mathrm{C}-2 \mathrm{~s}$ & $-Z / r$ & $n=2, l=0, m=0$ & $-Z^{2} \mu / 4$ & {$[2-\mu Z r] e^{-\mu Z r / 2}$} & $2^{-5 / 2} \pi^{-1 / 2}(\mu Z)^{3 / 2}$ & $\mu=1, Z=1$ \\
$\mathrm{C}-2 \mathrm{p}_{z}$ & $-Z / r$ & $n=2, l=1, m=0$ & $-Z^{2} \mu / 4$ & $z e^{-\mu Z r / 2}$ & $2^{-5 / 2} \pi^{-1 / 2}(\mu Z)^{5 / 2}$ & $\mu=1, Z=1$ \\
$\mathrm{C}-2 \mathrm{p}_{x}$ & $-Z / r$ & $n=2, l=1, m=1$ & $-Z^{2} \mu / 4$ & $\rho e^{-\mu Z r / 2} \cos \varphi$ & $2^{-5 / 2} \pi^{-1 / 2}(\mu Z)^{5 / 2}$ & $\mu=1, Z=1$ \\
$\mathrm{H}-000$ & $\frac{1}{2} \mu \omega^{2} r^{2}$ & $n_{x}=n_{y}=0, n_{z}=0$ & $\frac{3}{2} \omega$ & $e^{-\mu \omega r^{2} / 2}$ & $\pi^{-3 / 4}(\mu \omega)^{3 / 4}$ & $\mu=1, \omega=1$ \\
$\mathrm{H}-001$ & $\frac{1}{2} \mu \omega^{2} r^{2}$ & $n_{x}=n_{y}=0, n_{z}=1$ & $\frac{5}{2} \omega$ & $z e^{-\mu \omega r^{2} / 2}$ & $2^{1 / 2} \pi^{-3 / 4}(\mu \omega)^{5 / 4}$ & $\mu=1, \omega=1$ \\
\hline
\end{tabular}

Table 4.1: Properties of the potentials and their bound states that we used for testing the errors regarding the spatial discretization. Our main test potentials are the Coulomb potential (C) and the 3D harmonic oscillator potential $(\mathrm{H})$.

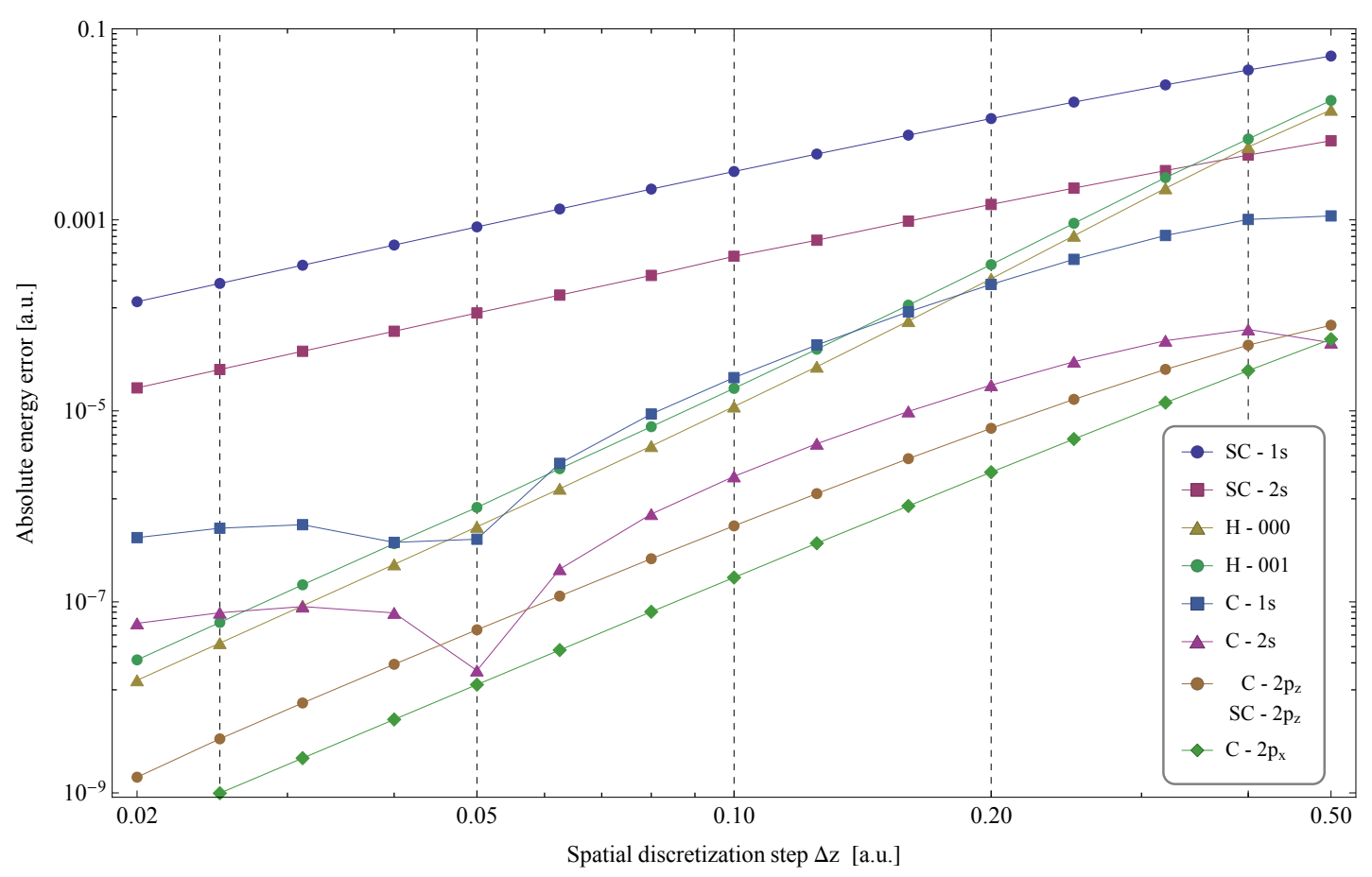

Figure 4.2: Absolute energy errors of stationary states listed in Table 4.1, on a log-log scale. The Coulomb eigenstates were tested with (C) and without (SC) applying the condition (4.26) at the origin. (In the case of the $2 p_{z}$ the error curves are within line thickness.) Curves for $1 s$ and $2 s$ show that our hybrid splitting algorithm decreases the errors with high-order even with the singular Coulomb potential, in the range $0.05 \lesssim \Delta z \lesssim 0.2$. 
Absolute energy errors of selected stationary states

\begin{tabular}{cccccccc}
\hline$\Delta z$ & 0.4 & 0.2 & 0.1 & 0.05 & 0.025 & Order $^{\text {Energy }_{0}}$ \\
\hline \hline $\mathrm{SC}-1 \mathrm{~s}$ & $3.71 \times 10^{-2}$ & $1.15 \times 10^{-2}$ & $3.21 \times 10^{-3}$ & $8.44 \times 10^{-4}$ & $2.16 \times 10^{-4}$ & 1.86 & -0.500 \\
$\mathrm{SC}-2 \mathrm{~s}$ & $4.77 \times 10^{-3}$ & $1.45 \times 10^{-3}$ & $4.15 \times 10^{-4}$ & $1.06 \times 10^{-4}$ & $2.71 \times 10^{-5}$ & 1.86 & -0.125 \\
$\mathrm{SC}^{-2} \mathrm{p}_{z}$ & $4.88 \times 10^{-5}$ & $6.57 \times 10^{-6}$ & $6.25 \times 10^{-7}$ & $5.10 \times 10^{-8}$ & $3.68 \times 10^{-9}$ & 3.42 & -0.125 \\
$\mathrm{C}-1 \mathrm{~s}$ & $1.01 \times 10^{-3}$ & $2.11 \times 10^{-4}$ & $2.23 \times 10^{-5}$ & $4.53 \times 10^{-7}$ & $5.91 \times 10^{-7}$ & 2.68 & -0.500 \\
$\mathrm{C}-2 \mathrm{~s}$ & $7.12 \times 10^{-5}$ & $1.86 \times 10^{-5}$ & $2.06 \times 10^{-6}$ & $1.91 \times 10^{-8}$ & $7.70 \times 10^{-8}$ & 2.46 & -0.125 \\
$\mathrm{C}-2 \mathrm{p}_{z}$ & $4.88 \times 10^{-5}$ & $6.57 \times 10^{-6}$ & $6.25 \times 10^{-7}$ & $5.10 \times 10^{-8}$ & $3.68 \times 10^{-9}$ & 3.42 & -0.125 \\
$\mathrm{C}-2 \mathrm{p}_{x}$ & $2.64 \times 10^{-5}$ & $2.28 \times 10^{-6}$ & $1.80 \times 10^{-7}$ & $1.36 \times 10^{-8}$ & $9.93 \times 10^{-10}$ & 3.67 & -0.125 \\
$\mathrm{H}-000$ & $5.79 \times 10^{-3}$ & $2.42 \times 10^{-4}$ & $1.11 \times 10^{-5}$ & $6.11 \times 10^{-7}$ & $3.70 \times 10^{-8}$ & 4.31 & +1.500 \\
$\mathrm{H}-001$ & $7.03 \times 10^{-3}$ & $3.40 \times 10^{-4}$ & $1.72 \times 10^{-5}$ & $9.75 \times 10^{-7}$ & $6.09 \times 10^{-8}$ & 4.20 & +2.500 \\
\hline
\end{tabular}

Table 4.2: A detailed table of the energy errors at specific values of $\Delta z$ denoted by vertical dashed lines in Fig. 4.2. The Order column gives the exponent of $\Delta z$, i.e. the order of error decrease, valid from step size $\Delta z=0.4$ to $\Delta z=0.025$, which characterizes the effective accuracy of the method in the given case. The Coulomb $2 p_{z}$ state also has the same error data set to the first 3 significant digits regardless whether or not the Robin boundary condition was used.

(the effective order is higher than 4 due to the fifth-order accurate second derivatives). For the Coulomb potential, things get considerably more elaborate. In the soft-Coulomb case (SC), the energies of the $l=0$ states converge with second-order (more precisely, with 1.86), meaning the method will not be sufficiently accurate or fast. However, for the true singular Coulomb case $(\mathrm{C})$, the accuracy of the $l=0$ states drastically improve by two orders of magnitude around $\Delta z=0.1$. On the other hand, the complicated step size dependence of the energy error, shown by the corresponding lines in Fig. 4.2, does not allow for a true effective order valid in the inspected range. However, our algorithm does decrease the error with high order (close to 4) when $0.05 \lesssim \Delta z \lesssim 0.2$, which is anyhow that range where the computation runtime is reasonable. The unusual step size dependence of the energy error below $\Delta z \lesssim 0.05$ for data sets C- $1 \mathrm{~s}, \mathrm{C}-2 \mathrm{~s}$ is due to finite differencing with high spatial accuracy applied to a nonanalytic problem $(\Psi$ is not continuously differentiable in the origin), along with the artificially high-order boundary condition.

The test cases with $l>0$ work reasonably well for the $m=0$ configuration both with $\mathrm{C}$ and $\mathrm{SC}$ : the $\mathrm{SC}-2 \mathrm{p}_{z}$ or the $\mathrm{C}-2 \mathrm{p}_{z}$ case has better (relative) accuracy than $\mathrm{H}-000$ but its order is only 3.42 as shown in Fig. 4.2. A slightly higher-order of 3.67 is achieved for C- $2 \mathrm{p}_{x}$ (the only test case with $m \neq 0$ ) which has the best accuracy of all computed eigenenergies in this section.

In summary, the numerical error of the hybrid splitting algorithm displays a high-order scaling with the spatial step size for the stationary states of the 3D hydrogen problem.

\subsubsection{Temporal accuracy: forced harmonic oscillator}

In the previous section we focused on the spatial errors in conjunction with the singular Coulomb potential, now we turn our attention to the total error of the hybrid splitting algorithm using a smooth time-dependent potential. This total error is composed typically of several terms related to the finite differences, to the Padé-approximation, to the factorization of the exponential operator, and to the short-time splitting of the evolution operator. 

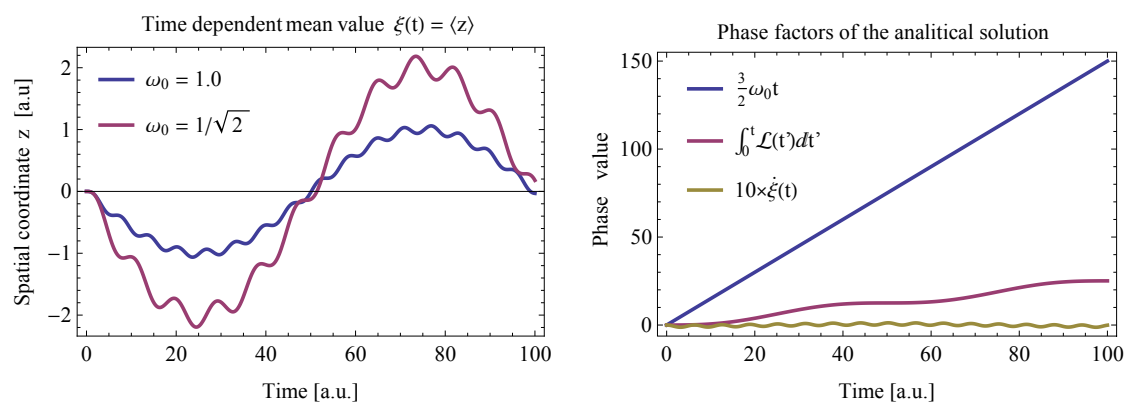

Figure 4.3: Time evolution of the analytical solution of the forced harmonic oscillator (see Appendix C): the expectation value of $z$ versus time (left), time dependence of the phase contributions (right). Parameters are the same as in Table 4.4, if not given explicitly.

\begin{tabular}{|c|c|c|}
\hline ID & Propagation method & Evaluations of $S_{2}$ \\
\hline \hline S2 & Second-order operator hybrid splitting scheme (Section 4.2.1). & 1 \\
\hline S4E3 & S2 based 4th-order iterative splitting scheme using eq. (2.34) with $n=4$. & 3 \\
\hline S6E5 & S2 based 6th-order iterative splitting scheme using eq. (2.35) with $n=6$. & 5 \\
\hline S6E9 & S2 based 6th-order iterative splitting scheme using eq. (2.34) with $n=6$. & 9 \\
\hline
\end{tabular}

Table 4.3: Test case definitions for comparison of the different high-order split-operator schemes.

We use the so-called forced harmonic oscillator (FHO) problem as the test case, defined by the potential $V(z, \rho, t)=\frac{1}{2} \mu \omega_{0}^{2}\left(z^{2}+\rho^{2}\right)+z F \sin \omega_{\mathrm{F}} t$, having a known $\Psi^{\mathrm{A}}(z, \rho, t)$ analytical solution, which we summarize in Appendix C. Here we only illustrate this time-dependent analytical solution in Fig. 4.3.

For error calculations, we compare the analytical and time-dependent wave functions at a fixed time instant by calculating the so-called mean-square error, which provides information about both the total phase and the amplitude related errors. We define the mean-square error by

$$
\operatorname{err}_{L^{2}}^{2}=2 \pi \int_{-\infty}^{\infty} \int_{0}^{\infty} \rho\left|\Psi^{\mathrm{A}}(z, \rho)-\Psi(z, \rho)\right|^{2} \mathrm{~d} \rho \mathrm{d} z
$$

We choose the mean-square error as the reference error type because (according to our experience) it is the most reliable evolution error quantifier at a fixed time instant $t$.

We tested our algorithm with different split-operators (listed in Table 4.3), and several $\Delta z$ and $\Delta t$ configurations to acquire a top-down view of the error properties of the hybrid splitting method.

The mean-square error of the different high-order split-operators based on our Crank-Nicolson scheme (CN5) can be found in Table 4.4, and also in Fig. 4.4: the standard convergence of the S2 scheme is very slow, and there is a drastic improvement using S4E3 on top of S2. Using either S6E9 or S6E5 does not yield much gain in accuracy compared to their higher numerical costs. Based on these, we propose to use the fourth-order split-operator method S4E3 in accordance with (2.34), along with our hybrid splitting scheme.

The lines corresponding to the different split-operators in Fig. 4.4 should exhibit the expected power scaling with $\Delta t$, this is only approximately the case and only above a threshold $\Delta t$. Below this threshold the total error is not reduced by decreasing $\Delta t$, because the evolution 
Calculated $L^{2}$ error values at $\Delta z=0.2$

\begin{tabular}{|c|c|c|c|c|c|c|c|}
\hline$\Delta t$ & 0.5 & 0.2 & 0.1 & 0.05 & 0.02 & 0.01 & 0.005 \\
\hline $\mathrm{S} 2$ & 1.37 & $4.89 \times 10^{-1}$ & $1.41 \times 10^{-1}$ & $4.80 \times 10^{-2}$ & $2.20 \times 10^{-2}$ & $1.83 \times 10^{-2}$ & $1.74 \times 10^{-2}$ \\
\hline S4E3 & $3.60 \times 10^{-1}$ & $4.65 \times 10^{-2}$ & $1.89 \times 10^{-2}$ & $1.72 \times 10^{-2}$ & $1.71 \times 10^{-2}$ & $1.70 \times 10^{-2}$ & $1.70 \times 10^{-2}$ \\
\hline S6E5 & $1.60 \times 10^{-1}$ & $2.15 \times 10^{-2}$ & $1.71 \times 10^{-2}$ & $1.70 \times 10^{-2}$ & $1.70 \times 10^{-2}$ & $1.70 \times 10^{-2}$ & $1.70 \times 10^{-2}$ \\
\hline S6E9 & $1.51 \times 10^{-1}$ & $2.10 \times 10^{-2}$ & $1.71 \times 10^{-2}$ & $1.70 \times 10^{-2}$ & $1.70 \times 10^{-2}$ & $1.70 \times 10^{-2}$ & $1.70 \times 10^{-2}$ \\
\hline \multicolumn{8}{|c|}{ Calculated $L^{2}$ error values at $\Delta z=0.1$} \\
\hline$\Delta t$ & 0.5 & 0.2 & 0.1 & 0.05 & 0.02 & 0.01 & 0.005 \\
\hline $\mathrm{S} 2$ & 1.83 & $5.95 \times 10^{-1}$ & $1.54 \times 10^{-1}$ & $3.95 \times 10^{-2}$ & $7.22 \times 10^{-3}$ & $2.61 \times 10^{-3}$ & $1.46 \times 10^{-3}$ \\
\hline S4E3 & $4.20 \times 10^{-1}$ & $3.87 \times 10^{-2}$ & $3.72 \times 10^{-3}$ & $1.24 \times 10^{-3}$ & $1.08 \times 10^{-3}$ & $1.08 \times 10^{-3}$ & $1.08 \times 10^{-3}$ \\
\hline S6E5 & $1.87 \times 10^{-1}$ & $1.02 \times 10^{-2}$ & $1.38 \times 10^{-3}$ & $1.09 \times 10^{-3}$ & $1.08 \times 10^{-3}$ & $1.08 \times 10^{-3}$ & $1.08 \times 10^{-3}$ \\
\hline S6E9 & $1.76 \times 10^{-1}$ & $9.50 \times 10^{-2}$ & $1.36 \times 10^{-3}$ & $1.09 \times 10^{-3}$ & $1.08 \times 10^{-3}$ & $1.08 \times 10^{-3}$ & $1.08 \times 10^{-3}$ \\
\hline \multicolumn{8}{|c|}{ Calculated $L^{2}$ error values at $\Delta z=0.05$} \\
\hline$\Delta t$ & 0.5 & 0.2 & 0.1 & 0.05 & 0.02 & 0.01 & 0.005 \\
\hline $\mathrm{S} 2$ & 2.38 & $7.21 \times 10^{-1}$ & $1.84 \times 10^{-1}$ & $4.62 \times 10^{-2}$ & $7.46 \times 10^{-3}$ & $1.93 \times 10^{-3}$ & $5.43 \times 10^{-4}$ \\
\hline S4E3 & $4.95 \times 10^{-1}$ & $4.35 \times 10^{-2}$ & $3.33 \times 10^{-3}$ & $3.11 \times 10^{-4}$ & $8.85 \times 10^{-5}$ & $8.26 \times 10^{-5}$ & $8.22 \times 10^{-5}$ \\
\hline S6E5 & $2.21 \times 10^{-1}$ & $1.19 \times 10^{-2}$ & $6.71 \times 10^{-4}$ & $1.19 \times 10^{-4}$ & $8.30 \times 10^{-5}$ & $8.22 \times 10^{-5}$ & $8.22 \times 10^{-5}$ \\
\hline S6E9 & $2.07 \times 10^{-1}$ & $1.11 \times 10^{-2}$ & $6.37 \times 10^{-4}$ & $1.18 \times 10^{-4}$ & $8.29 \times 10^{-5}$ & $8.22 \times 10^{-5}$ & $8.22 \times 10^{-5}$ \\
\hline
\end{tabular}

Table 4.4: Mean-square errors of the forced harmonic oscillator with different $\Delta z(=\Delta \rho), \Delta t$ and split-operator configurations. All calculations were carried out in a box of $-10 \leq z \leq 10$ and $0 \leq \rho \leq 8$ with propagation parameters $\omega_{\mathrm{F}}=2 \pi / 100, F=1, \mu=1, \omega_{0}=1$, launched from the corresponding ground state $(\mathrm{H}-000)$. All error values are calculated at the time $t=100$.
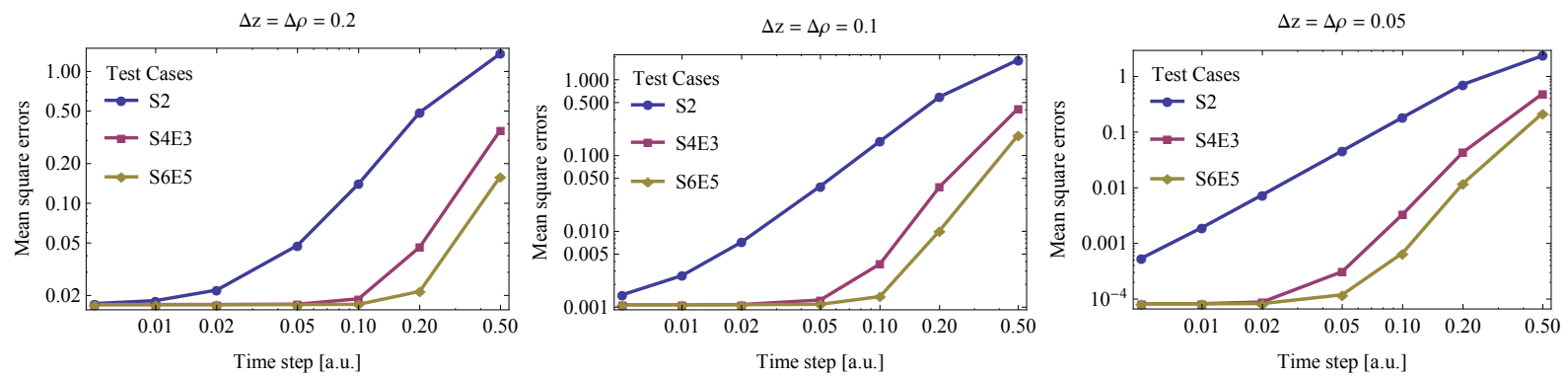

Figure 4.4: Mean-square errors as the function of the time step, on a log-log scale, computed with different split-operator methods and spatial discretization steps, corresponding to Table 4.4. These plots clearly show the existence of a threshold value of $\Delta t$, below which the total error is not reduced anymore.

Calculated $L^{2}$ error values of CN3 based S4E3 method

\begin{tabular}{cccccccc}
\hline$\Delta t$ & 0.5 & 0.2 & 0.1 & 0.05 & 0.02 & 0.01 & 0.005 \\
\hline \hline$\Delta z=0.2$ & $7.17 \times 10^{-1}$ & $4.33 \times 10^{-1}$ & $4.09 \times 10^{-1}$ & $4.07 \times 10^{-1}$ & $4.07 \times 10^{-1}$ & $4.07 \times 10^{-1}$ & $4.07 \times 10^{-1}$ \\
$\Delta z=0.1$ & $5.64 \times 10^{-1}$ & $1.88 \times 10^{-1}$ & $1.56 \times 10^{-1}$ & $1.54 \times 10^{-1}$ & $1.54 \times 10^{-1}$ & $1.54 \times 10^{-1}$ & $1.54 \times 10^{-1}$ \\
$\Delta z=0.05$ & $5.47 \times 10^{-2}$ & $9.72 \times 10^{-2}$ & $5.82 \times 10^{-2}$ & $5.54 \times 10^{-2}$ & $5.52 \times 10^{-2}$ & $5.52 \times 10^{-2}$ & $5.52 \times 10^{-2}$
\end{tabular}

Table 4.5: Mean-square errors of a commonly used propagation scheme, to be compared with the data of Table 4.4. All the parameters are the same as in Table 4.4. Comparison of the last columns shows one, two and three orders of magnitude accuracy increase in favor of the five-point discretization, as $\Delta z$ is decreased. 
error is dominated by the finite differences: the error magnitudes can even be predicted as the product of the stationary energy error (Table 4.2) and the propagation time interval.

One objective of our research was to design a real space finite difference algorithm that achieves fourth-order accuracy in the spatial step size and, most importantly, that is capable to include both the singular coordinate $\rho=0$ and the singular Coulomb potential directly. Therefore, it is interesting to globally benchmark the results against a second-order 3-point CrankNicolson finite difference scheme (CN3), which means we degraded the core algorithm to use three-point finite differences (A.2) of Appendix A in S2.

We repeated the main tests with this $\mathrm{CN} 3$ method combined with the split-operator configuration S4E3 (discussed above). The $\mathrm{CN} 3$ results are shown in Table 4.5 and are to be compared to the results with CN5 in Table 4.4. Both the CN3 and CN5 schemes display the expected order scaling of the error with $\Delta z$. Comparing the $L^{2}$ error at $\Delta t=0.005$ (which is already below the threshold $\Delta t$ ), our fourth-order discretization has one, two and three orders of magnitudes smaller errors at spatial step sizes $\Delta z=0.2,0.1,0.05$, respectively. Thus, one should always use (at least) the CN5 scheme unless the exotic nature of the problem prevents high-order discretization.

In summary, our hybrid splitting propagation method is suitable for numerical simulations with time-dependent Hamiltonians, and it is capable of high-order scaling with $\Delta t$, once it is incorporated into the proper high-order evolution operator formulas.

\subsubsection{Hydrogen atom in an external field}

Finally, we conducted a test similar to the real world applications by simulating the hydrogen atom in an external laser field. Now, we compare our hybrid splitting based CN5 implementation, including Coulomb potential and the Coulomb boundary condition (CN5-C) against the commonplace $\mathrm{CN} 3$ discretization with the best soft-Coulomb potential approximation allowed by the spatial grid (CN3-SC), which is the same approximation that we tested in Section 4.3.1. We did not use the full 2D CN3 method, but we applied the hybrid splitting scheme using the $\mathrm{CN} 3$ equations just like the case of the FHO.

The external field was parametrized as $\mathscr{E}_{z}(t)=F \sin \omega t$. The simulated time was $t \in[0,100]$, and it consisted of only one field cycle, by setting $\omega=2 \pi / 100$ in atomic units. The field amplitude was $F=0.08$, with simulation box size $z \in[-250,250], \rho \in[0,100]$ to contain the escaping electron waves. The initial state was the $1 s$ ground state, found by imaginary time propagation method to remove spurious ionization components. We use the S4E3 high-order split-operator formula as indicated in the last section.

We show the result of this simulation in Fig. 4.5 by a density plot of the logarithm of the absolute square of the wave function, in a plane containing the $z$ axis. The white waves on the right of the spherical peak, bound by the Coulomb potential of the nucleus, are ca. 4-5 order smaller in density. These waves have to be computed very accurately, since they contribute the most to the time dependence of the dipole moment, which in turn is of fundamental importance regarding the $\mathrm{HHG}$ and the creation of attosecond light pulses.

The tests to compare CN5-C with CN3-SC were run with $\Delta \rho=\Delta z$ and with time step $\Delta t=0.01$ if not indicated explicitly. We quantified the accuracy of the solutions with the error 


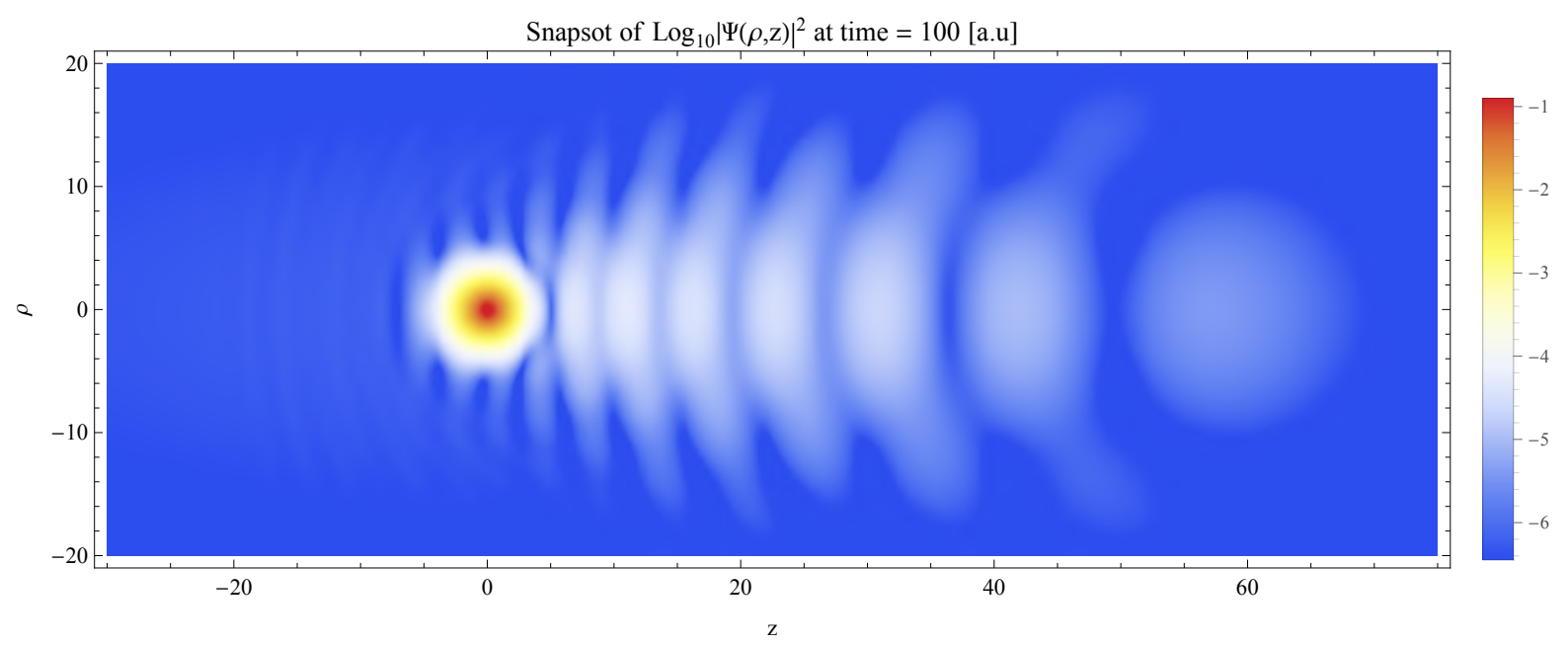

Figure 4.5: Density plot of the logarithm of the absolute square of the wave function, in a plane containing the $z$ axis, at the end of the simulation described in Section 4.3.3. Note that the white waves are 4-5 orders of magnitude smaller than the spherical peak.

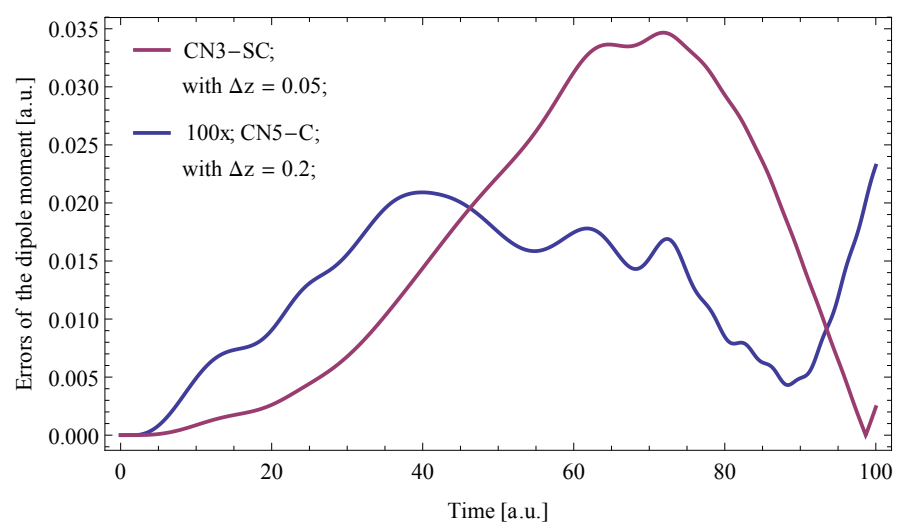

Figure 4.6: Comparison of the errors of $\langle z\rangle$, during the time propagation described in Section 4.3.3, using the method CN5-C with $\Delta z=0.2$ a.u. (blue line) and using the method CN3-SC with $\Delta z=0.05$ a.u. (purple line). Despite the larger spatial step used with CN5-C, its error is two orders of magnitude smaller, therefore it is shown here with a 100 times magnification.

of the expectation value $\langle z\rangle$, i.e. the magnitude of the time-dependent dipole moment. Unlike the FHO example, analytic solution is not available this time, so we use a converged solution to determine numerical errors, that is we compare the results with a much more accurate numerical solution obtained using smaller $\Delta t, \Delta z$ discretization steps.

The results, shown in Fig. 4.6 are as expected: the error of the CN5-C scheme with $\Delta z=0.2$ is smaller by two orders of magnitude than that of the CN3-SC scheme with $\Delta z=0.05$. Based on this, we estimate the performance difference as follows. Due to the second-order convergence of the CN3-SC scheme, $\Delta z \approx 0.005$ is needed to achieve the accuracy of the CN5-C with $\Delta z=0.2$, which means a factor of 1600 in the number of spatial gridpoints, implying a factor of $800-$ 1600 in runtime. That is, the CN5-C scheme is ca. 1000 times more effective than the CN3-SC. Regarding absolute accuracy, the magnitude of the error of $\langle z\rangle(t)$ using CN5-C with $\Delta z=0.2$ is smaller than the $L^{2}$ error of the FHO at the time instant $t=100$, as can be seen in Table 4.4. 


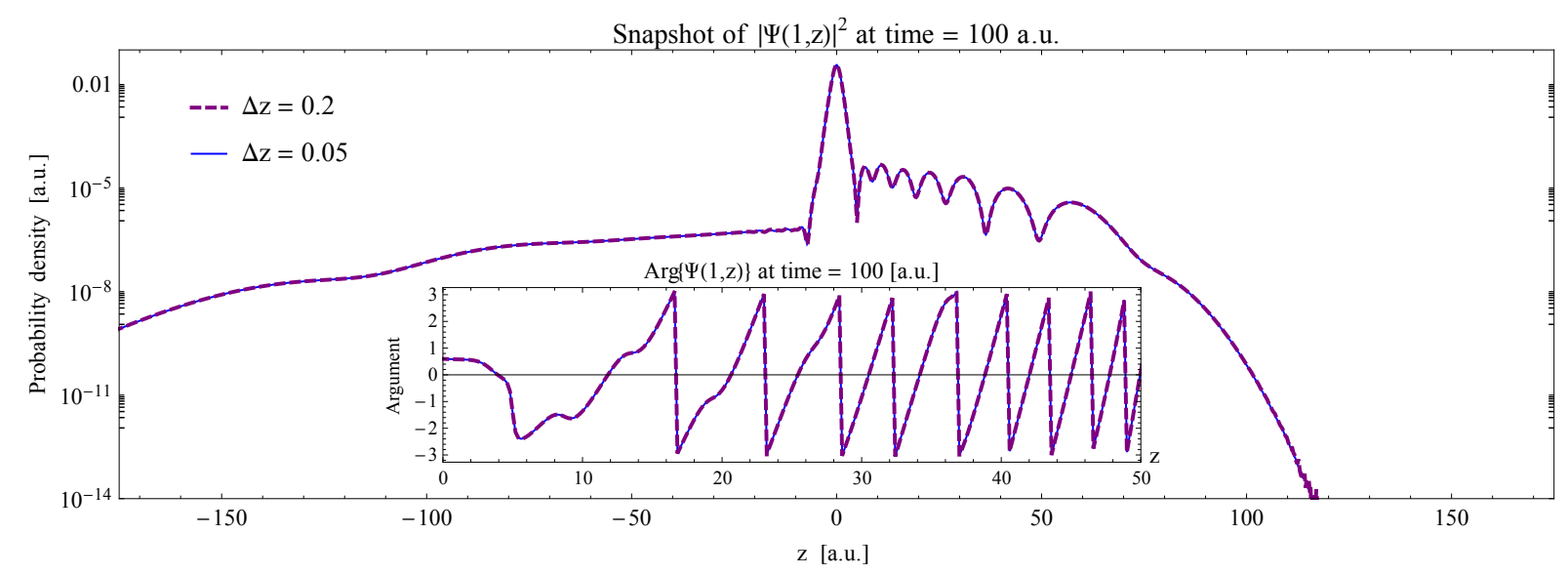

Figure 4.7: Probability density and phase (in the inset) along a z-line section at $\rho=1$, at the end of the time propagation described in Section 4.3.3, computed using the CN5-C method with two different values of $\Delta z$, as indicated in the figure. The excellent fit of the two curves shows that the solution can be considered converged already at $\Delta z=0.2$.

Regarding the pointwise convergence of the solution with the CN5-C method, we compare the density and the phase along a $z$-line section at $\rho=1$, computed with two different values of $\Delta z$. These plots, shown in Fig. 4.7, convincingly show that a converged numerical solution is obtained already at $\Delta z=0.2$. Note that the accurate calculation of the phase is important in certain areas of strong-field physics, regarding e.g. exit momentum calculations $[15,108]$ in optical tunneling or regarding phase space methods [31, 32, 109-111], where usually the Wigner function is computed from the wave function and this is then further analysed for the physical interpretation of the process.

\subsection{Summary}

In this chapter we presented an efficient algorithm capable of the direct numerical solution of the three-dimensional time-dependent Schrödinger equation in strong-field circumstances, assuming axial symmetry in cylindrical coordinates. We choose a high-order finite difference representation in the spatial coordinate domain. The main feature of the algorithm is that it is capable to accurately handle singular Coulomb potentials besides any smooth potential of the form $V(z, \rho)$, We implemented all singularities that can be reduced to the form $1 / \rho$ or $1 / r$ via Neumann and Robin boundary conditions at the $\rho=0$ axis. The axial symmetry and the availability of the Cartesian $z$-axis makes it easy to investigate reduced electron dynamics in contrast to spherical coordinate representations.

We based our algorithm on the a high-order finite difference representation in the spatial derivatives and the split-operator approximation of the short-time evolution operator. Due to the nonseparability of the Coulomb-problem in cylindrical coordinates, we constructed a special second-order operator splitting scheme called hybrid splitting method. This splits the Hamiltonian matrix directionwise like the traditional methods, but the innermost region near $\rho=0$ is excluded: here the full 2D Crank-Nicolson equations are used. This means that there are many decoupled 1D Schrödinger equations in the $z$ direction, and a 2D Schrödinger equation 
with a special block pentadiagonal pattern of the coefficient matrix, which has to be taken advantage of for maximum efficiency, like in our hybrid splitting solver algorithm. Thread based parallelization is supported throughout, and we also gave a way to evaluate the decoupled $1 \mathrm{D}$ Schrödinger equations with high spatial accuracy and efficiency.

We thoroughly investigated the spatial discretization related errors in an optimal discretization parameter range, determining detailed accuracy characteristics with or without Coulomb potential. We also verified the considerably increased accuracy for numerical simulations forced oscillator. We concluded that high-order (meaning at least 4th-order) split-operator formula should be used in practice, accompanying the hybrid splitting algorithm. In order to demonstrate the fourth-order spatial accuracy and performance of our hybrid splitting algorithm also in a simulation close to the planned applications, we computed the solution for a hydrogen atom in a strong time-dependent electric field of one sine period. The important waves in the probability density, having an amplitude of $10^{-4}-10^{-5}$ relative to the peak value, were obtained accurately and efficiently. 


\section{ChAPTER 5}

\section{Quantum entanglement in strong-field ionization}

We have already discussed in Section 1.3 that the quantum state of the interacting two-body system of an electron and its parent ion-core involves quantum entanglement between these two particles. A straightforward question is, how strong-field ionization affects this electron - ion-core entanglement in a real atom? In the present chapter, we report our new results on this process: we investigate the hydrogen atom in 3 spatial dimensions, using the true Coulomb potential by performing accurate numerical simulations. These results are based on our publication [P2].

In Section 5.2, we present the details of our entanglement calculations in 3 dimensions which is based on the directionally reduced dynamics. Amongst others, we introduce the spatial entropy of the wave function and the correlation entropies within the directional subspaces. Using these directional entropies, we propose an approximate formula to quantify the total electron - ion-core entanglement we actually seek for. In Section 5.3, we discuss the results of our preliminary investigation based on our one-dimensional model corresponding to our earlier publication [A1]: based on a simple one-dimensional model, we show that the time evolution of this quantum entanglement shows interesting features. In Section 5.4 we present our numerical results on the temporal evolution of quantum entanglement during the strong-field ionization process in 3 dimensions. We show how do the specific quantities, including several different entropies, reflect the system's behavior, and we investigate in detail their dependence on the intensity and the carrier-envelope phase difference (CEP) of the laser pulse.

\subsection{Theoretical model}

The theoretical model is entirely based on the equations of Section 1.3, in which we discussed the two-body description of a single-electron atom. The solution of the problem is given in relative (r) and center-of-mass $\left(\mathbf{r}_{0}\right)$ coordinates as $\Psi(\mathbf{r}, t) \Psi_{0}\left(\mathbf{r}_{0}, t\right)$ which equals the full wave function of the system $\Psi_{e c}\left(\mathbf{r}_{e}, \mathbf{r}_{c}, t\right)$, where the electron $\left(\mathbf{r}_{e}\right)$ and ion-core $\left(\mathbf{r}_{c}\right)$ coordinates are given by the coordinate transformation (1.20).

For the relative part, we solve the axially symmetric time-dependent Schrödinger equation (1.6) in cylindrical coordinates to get the wave function $\Psi(z, \rho, t)$. We shall seek solutions that start from the ground state of hydrogen atom $(Z=1)$ at $t=0$ using singular Coulomb potential 
at the origin as in Eq. (1.9). This wave function does not depend on the azimuthal angle $\varphi$ and this remains valid for the solution at any later time. For the solution in 3D, we used our hybrid splitting method, discussed in Chapter 4. Using this method we can achieve reasonable accuracy already at the uniform spatial discretization step size $\Delta z=\Delta \rho=0.2$, which may seem to be rough at first sight, but it turns out to be sufficient in view of the large extension of the ionized part of the relative wave function. For all the simulations presented in this chapter, the initial state is the $1 \mathrm{~s}$ ground state with energy $E_{0}=-\mu / 2$ and $\mu=0.999456$, corresponding to the reduced mass of the proton-electron system.

For the center-of-mass part, we assume that $\Psi_{0}$ is initially a localized Gaussian wave packet at rest in coordinate space, which yields the solution of (1.24) as

$$
\Psi_{0}\left(\mathbf{r}_{0}, t\right)=\left(\frac{\sigma_{0} / \sqrt{\pi}}{\sigma_{0}^{2}+i \frac{t}{M}}\right)^{3 / 2} \exp \left(-\frac{\mathbf{r}_{0}^{2}}{2\left(\sigma_{0}^{2}+i \frac{t}{M}\right)}\right)
$$

We set the parameter $\sigma_{0}=1$ based on the size of a hydrogen atom (i.e. a Bohr radius). This is a well-known free wave packet, which is separable according to the directions $x_{0}, y_{0}, z_{0}$ and has root-mean-square deviations of the center-of-mass coordinates in each direction as

$$
\sigma_{x_{0}}(t)=\sigma_{y_{0}}(t)=\sigma_{z_{0}}(t)=\sqrt{\sigma_{0}^{2}+t^{2} / M^{2} \sigma_{0}^{2}}
$$

which is to be evaluated in the time interval given by the duration $T_{\max }$ of the exciting pulse. The typical value of the latter in strong-field experiments is $T_{\max }=300$ a.u. corresponding to a few femtoseconds, used also in our simulations. Due to the large value of $M \approx 1837$, the spreading during the interaction is negligible: around $1.3 \%$ of the original width, which will help us to make the effect of the laser pulse on the quantum entropies more visible in Section 5.4 .

\subsection{Entanglement calculation}

Now, if we try to apply the standard entanglement quantification procedure given by Eqs. (3.2) - (3.9) of Section 3.1 to the discrete function $\Psi_{e c}\left(\mathbf{r}_{e}, \mathbf{r}_{c}, t\right)$ in $3 \mathrm{D}$, we can quickly conclude that the array size of the discretized density matrices involved will be prohibitively high. If we try to perform the reduction to $\varrho_{c}\left(\mathbf{r}_{c}^{\prime}, \mathbf{r}_{c}, t\right)$ and then to calculate the von Neumann entropy $S_{N}(t)$, we face effectively an $\sim N^{9}$ operations count per one value of $S_{N}(t)$, where $N$ is the characteristic number of gridpoints of a spatial coordinate. Using a typical setup, we needed at least $N=10^{3}$ gridpoints to contain the ionized electron waves. So if we make an optimistic guess that the execution takes about 1 second per $10^{9}$ operations, then we obtain a runtime of $32 \times 10^{9}$ years. This makes the computation according to the standard approach of Section 3.1 practically impossible, thus we have to find a viable approximation. 


\subsubsection{Directionally reduced dynamics}

We propose to circumvent the prohibitively large numerical load of the problem by restricting ourselves to only one coordinate direction (at a time), i.e. to utilize the directionally reduced dynamics. Since the system is axially symmetric around the polarization direction of the laser field (the axis $\rho=0$ at all times), it seems to be plausible to assume that the interesting physics happens in this direction. However, we are also going to use the information contained in the perpendicular directions.

The directionally reduced density matrices of the relative part are the following

$$
\begin{gathered}
\varrho_{x}\left(x^{\prime}, x\right)=\iint \Psi^{*}\left(x^{\prime}, y, z\right) \Psi(x, y, z) \mathrm{d} z \mathrm{~d} y, \\
\varrho_{z}\left(z^{\prime}, z\right)=2 \pi \int \Psi^{*}\left(z^{\prime}, \rho\right) \Psi(z, \rho) \rho \mathrm{d} \rho
\end{gathered}
$$

and because of the axial symmetry we have for the $y$ direction

$$
\varrho_{y}=\varrho_{x} .
$$

The directionally reduced density matrix of the center-of-mass part is

$$
\varrho_{0, z}\left(z_{0}^{\prime}, z_{0}\right)=2 \pi \int \Psi_{0}^{*}\left(z_{0}^{\prime}, \rho_{0}\right) \Psi_{0}\left(z_{0}, \rho_{0}\right) \rho_{0} \mathrm{~d} \rho_{0} .
$$

Due to the assumed Gaussian form (5.1) it is a pure state density matrix which can be calculated analytically. Again, because of symmetry we have in the other directions

$$
\varrho_{0, z}=\varrho_{0, y}=\varrho_{0, x} .
$$

In addition to this, only the density matrix of the relative part must be evaluated numerically.

After we have completed these, we will utilize that the separability is true in each direction:

$$
\varrho_{e c, x}=\varrho_{x} \otimes \varrho_{0, x} \text { and } \varrho_{e c, z}=\varrho_{z} \otimes \varrho_{0, z}
$$

Finally, we apply the necessary coordinate transformation (1.20) to (5.8), then the $x$ and $z$ directional two-particle reduced density matrices are given by

$$
\begin{gathered}
\varrho_{e c, x}\left(x_{e}^{\prime}, x_{e}, x_{c}^{\prime}, x_{c}\right)=\varrho_{x}\left(x_{e}^{\prime}-x_{c}^{\prime}, x_{e}-x_{c}\right) \varrho_{0, x}\left(\alpha_{e} x_{e}^{\prime}+\alpha_{c} x_{c}^{\prime}, \alpha_{e} x_{e}+\alpha_{c} x_{c}\right), \\
\varrho_{e c, z}\left(z_{e}^{\prime}, z_{e}, z_{c}^{\prime}, z_{c}\right)=\varrho_{z}\left(z_{e}^{\prime}-z_{c}^{\prime}, z_{e}-z_{c}\right) \varrho_{0, z}\left(\alpha_{e} z_{e}^{\prime}+\alpha_{c} z_{c}^{\prime}, \alpha_{e} z_{e}+\alpha_{c} z_{c}\right) .
\end{gathered}
$$

Then, we calculate the subsystem density matrices as in Section 3.1. Therefore, the onedimensional reduced density matrices of the ion-core coordinates are

$$
\varrho_{c, x}\left(x_{c}^{\prime}, x_{c}\right)=\operatorname{Tr}_{e}\left[\varrho_{e c, x}\right]=\int \varrho_{e c, x}\left(x_{e}, x_{e}, x_{c}^{\prime}, x_{c}\right) \mathrm{d} x_{e}
$$




$$
\varrho_{c, z}\left(z_{c}^{\prime}, z_{c}\right)=\operatorname{Tr}_{e}\left[\varrho_{e c, z}\right]=\int \varrho_{e c, z}\left(z_{e}, z_{e}, z_{c}^{\prime}, z_{c}\right) \mathrm{d} z_{e},
$$

and, similarly, we have for the electron coordinates

$$
\begin{gathered}
\varrho_{e, x}\left(x_{e}^{\prime}, x_{e}\right)=\operatorname{Tr}_{c}\left[\varrho_{e c, x}\right]=\int \varrho_{e c, x}\left(x_{e}^{\prime}, x_{e}, x_{c}, x_{c}\right) \mathrm{d} x_{c}, \\
\varrho_{e, z}\left(z_{e}^{\prime}, z_{e}\right)=\operatorname{Tr}_{c}\left[\varrho_{e c, z}\right]=\int \varrho_{e c, z}\left(z_{e}^{\prime}, z_{e}, z_{c}, z_{c}\right) \mathrm{d} z_{c} .
\end{gathered}
$$

In this way we have the building blocks of the two-body Coulomb system as six pieces of onedimensional reduced density matrices, which can already be computed in a reasonable amount of time.

\subsubsection{Correlation quantification per direction}

From these reduced density matrices we can calculate several quantum entropies, and each has a specific interesting aspect, we will list them in the following. For simplicity, we use mainly formulas of the von Neumann entropy, and we usually drop its subscript $N$.

Spatial entropy: this can be calculated from the reduced density matrix of the relative part as

$$
S_{z}(t)=S_{N}\left(\varrho_{z}(t)\right)=-\sum_{k} \lambda_{k}^{(z)}(t) \ln \lambda_{k}^{(z)}(t)
$$

where $\lambda_{k}^{(z)}(t)$ are the eigenvalues of $\varrho_{z}(t)$. We shall call (5.15) "spatial entanglement" measure, because it quantifies the entanglement between the relative coordinates $z, \rho$ (or the directional nonseparability of the strong-field process) according to the theory of pure bipartite systems. It is also the entropy of the two-dimensional subspace

$$
S_{e c, z}(t)=S_{N}\left(\varrho_{z}(t) \otimes \varrho_{0, z}(t)\right)=S_{z}(t)
$$

since $S_{N}\left(\varrho_{0, z}(t)\right)=0$. We also note that using

$$
S_{x}(t)=S_{N}\left(\varrho_{x}(t)\right)=-\sum_{k} \lambda_{k}^{(x)}(t) \ln \lambda_{k}^{(x)}(t)
$$

where $\lambda_{k}^{(x)}(t)$ are the eigenvalues of $\varrho_{x}(t)$, is also an option as a "spatial entanglement" measure. However, since the laser polarization coincides with the $z$ axis, it is the most interesting to know the nonseparability between the $z$ and $x \otimes y$ subspaces, therefore, we will prefer the use of $S_{z}(t)$.

Average mutual entropy per direction: as introduced by the formula (3.23), the (average) quantum mutual entropy is a true nonseparability and correlation measure generally, which can be used between the single-coordinate subsystems of the electron and the ion-core in a given direction. They are written along the $x$ and $z$ direction as

$$
\bar{S}\left(x_{e}: x_{c}, t\right)=\frac{1}{2}\left[S_{e, x}(t)+S_{c, x}(t)-S_{x}(t)\right],
$$




$$
\bar{S}\left(z_{e}: z_{c}, t\right)=\frac{1}{2}\left[S_{e, z}(t)+S_{c, z}(t)-S_{z}(t)\right] .
$$

To remind, these are exact formulas for pure bipartite states. However, these measures combine classical and entanglement related correlations otherwise, and in order to apply them as entanglement measures (per direction), we need to look at all of their constituent parts. It is also interesting how the conditional entropies behave.

Core entropies per direction: as we will show below, the following quantum entropies

$$
\begin{aligned}
& S_{c, x}(t)=S_{N}\left(\varrho_{c, x}(t)\right)=-\sum_{k} \lambda_{k}^{(c, x)}(t) \ln \lambda_{k}^{(c, x)}(t), \\
& S_{c, z}(t)=S_{N}\left(\varrho_{c, z}(t)\right)=-\sum_{k} \lambda_{k}^{(c, z)}(t) \ln \lambda_{k}^{(c, z)}(t),
\end{aligned}
$$

where $\lambda_{k}^{(c, x)}(t), \lambda_{k}^{(c, z)}(t)$ are the eigenvalues of $\varrho_{c, x}(t)$ and $\varrho_{c, z}(t)$ respectively, measure approximately the particle-particle correlation directionwise. The reason is the following in case of direction $z$ : because of the orders of magnitude of mass difference present in the coordinate transformation (5.10), the reduced density matrix $\varrho_{c, z}$ will be close to $\varrho_{0, z}$. This causes that only a tiny fraction $\left(m_{e} / M\right)$ of the entropy $S_{z}(t)$ of $z_{e} \otimes z_{c}$ is transferred to subsystem $z_{c}$, because the mass difference suppresses the eigenvalues and eigenvectors of $\varrho_{z}$. Knowing that $\varrho_{0, z}$ is a pure state density matrix with zero entropy, we conclude that additional surplus values in entropy $S_{c, z}(t)$ quantify the particle-particle correlation along the $z$ direction. In other words, it is the nonseparability between $z_{c}$ and $z_{e}$, which yet to be called entanglement. The same considerations also apply to the $x$ direction. Because these Neumann entropies are actually correlation entropies in this case, we expect them look really similar to the respective quantum mutual entropies. For sake of completeness, we note that the entropies (5.20) and (5.21) are also entanglement entropies of two special bipartitions of the six coordinate quantum system, namely $x_{c}$ against all the other coordinates and $z_{c}$ against all the other coordinates, respectively.

Electron entropies per direction: these are also of importance related to the conditional entropies, and the distinction of quantum versus classical correlations. Similarly, they are also special entanglement entropies of two bipartitions of the six coordinate quantum system in a similar manner as the core entropies per direction. They are calculated as

$$
\begin{aligned}
& S_{e, x}(t)=S_{N}\left(\varrho_{e, x}(t)\right)=-\sum_{k} \lambda_{k}^{(e, x)}(t) \ln \lambda_{k}^{(e, x)}(t), \\
& S_{e, z}(t)=S_{N}\left(\varrho_{e, z}(t)\right)=-\sum_{k} \lambda_{k}^{(e, z)}(t) \ln \lambda_{k}^{(e, z)}(t),
\end{aligned}
$$

where $\lambda_{k}^{(e, x)}(t), \lambda_{k}^{(e, z)}(t)$ are the eigenvalues of $\varrho_{e, x}(t)$ and $\varrho_{e, z}(t)$ respectively. We note that although $\varrho_{e}$ and $\varrho_{c}$ must have the same eigenvalues, this won't be true for the reduced density matrices $\varrho_{e, z}$ and $\varrho_{c, z}$ in direction $z$ if the values of $S_{z}(t)$ are not negligible. (Same goes for the $x$ direction.) Then the coordinate transformation (5.10) causes that the major fraction $\left(m_{c} / M\right)$ of the entropy $S_{z}(t)$ of $z_{e} \otimes z_{c}$ is transferred to subsystem $z_{e}$, because the reduced density matrix $\varrho_{e, z}$ will be close to $\varrho_{z}$. Based on the quantum information theoretic properties of the Neumann entropies, this spurious eigenvalue contribution can be extracted, but not completely. 
This "eigenvalue extraction" we refer to can be realized by the $S_{e, z}(t)-S_{z}(t)$ entropy subtraction, which are the single-direction negative quantum conditional entropies of the core- $x$ and core- $z$ reduced density matrices:

$$
\begin{aligned}
& -S\left(x_{c} \mid x_{e}, t\right)=S_{e, x}(t)-S_{x}(t), \\
& -S\left(z_{c} \mid z_{e}, t\right)=S_{e, z}(t)-S_{z}(t) .
\end{aligned}
$$

Since they are related to the correlation one way or the other, from the above reasoning it follows that $-S\left(x_{c} \mid x_{e}, t\right)$ and $-S\left(z_{c} \mid z_{e}, t\right)$ should be positive and similar to $S_{c, x}(t)$ and $S_{c, z}(t)$ and therefore also to their mutual entropy, respectively. Based on this reasoning we will see that the subsystems $x_{e} \otimes x_{c}$ and $z_{e} \otimes z_{c}$ are mainly subject to quantum entanglement (in accordance with (3.15)), not classical correlation (also present), which we show in Section 5.4.3.

Upper bound of the core entropy: using the strong subadditivity of the Neumann entropy, an upper bound can be given for the true 3D electron - ion-core entanglement as

$$
S_{e}(t)=S_{c}(t) \leq S_{c, z}(t)+2 S_{c, x}(t)=S_{\text {bound }}(t),
$$

where the one-dimensional ion-core entropies were substituted into the subadditivity relation (3.10), because they tend to be smaller than those of the electrons and because of the physical reasons outlined above. The equation (5.26) serves also as a good analytical criteria that we should fulfill with an approximate formula for electron - ion-core entanglement.

\subsubsection{Approximation of the entanglement}

Now we introduce our approximate entanglement measure, which is one of the main purposes of this chapter.

We approximate the pure state of our six-dimensional quantum system by replacing it with

$$
\varrho_{e c}^{(\mathrm{sep})}(t)=\varrho_{e c, x}(t) \otimes \varrho_{e c, y}(t) \otimes \varrho_{e c, z}(t),
$$

which is separable directionwise but it includes the $\varrho_{e c, x}\left(x_{e}^{\prime}, x_{e}, x_{c}^{\prime}, x_{c}\right), \varrho_{e c, y}\left(y_{e}^{\prime}, y_{e}, y_{c}^{\prime}, y_{c}\right)$, and $\varrho_{e c, z}\left(z_{e}^{\prime}, z_{e}, z_{c}^{\prime}, z_{c}\right)$ two-dimensional reduced density matrices which contain all the pair correlations between the coordinates $x_{e}-x_{c}, y_{e}-y_{c}, z_{e}-z_{c}$, respectively. (Because of the symmetry, the physics in the subspaces $x$ and $y$ are identical, so (5.5) is true.) Then we obtain the entropy of $\varrho_{e c}^{\text {(sep) }}$ from the additivity of the Neumann entropy (valid for separable systems), and using that $S_{e c, j}(t)=S_{j}(t)+S_{0, j}(t), \varrho_{e c, j}=\varrho_{j} \otimes \varrho_{0, j}, j=x, y, z$ as

$$
S_{e c}^{(\mathrm{sep})}(t)=S_{x}(t)+S_{y}(t)+S_{z}(t)
$$

The single-particle ion-core and electron reduced density matrices read

$$
\begin{aligned}
& \varrho_{e}^{(\mathrm{sep})}(t)=\varrho_{e, x}(t) \otimes \varrho_{e, y}(t) \otimes \varrho_{e, z}(t), \\
& \varrho_{c}^{(\mathrm{sep})}(t)=\varrho_{c, x}(t) \otimes \varrho_{c, y}(t) \otimes \varrho_{c, z}(t),
\end{aligned}
$$


with the standard definitions $(j=x, y, z)$ :

$$
\varrho_{e, j}(t)=\operatorname{Tr}_{c}\left[\varrho_{e c, j}(t)\right] \text { and } \varrho_{c, j}(t)=\operatorname{Tr}_{e}\left[\varrho_{e c, j}(t)\right]
$$

For the entropies of these, the following hold:

$$
\begin{aligned}
& S_{e}^{(\mathrm{sep})}(t)=S_{e, x}(t)+S_{e, y}(t)+S_{e, z}(t), \\
& S_{c}^{(\mathrm{sep})}(t)=S_{c, x}(t)+S_{c, y}(t)+S_{c, z}(t) .
\end{aligned}
$$

We propose to quantify the total entanglement between $e$ and $c$ based on the average mutual entropy (3.23) as

$$
\bar{S}_{e c}(e: c, t)=\frac{1}{2}\left[S_{e}^{(\mathrm{sep})}(t)+S_{c}^{(\mathrm{sep})}(t)-S_{e c}^{(\mathrm{sep})}(t)\right] .
$$

After rearranging the terms and using symmetry relations (5.5), (5.7) we obtain

$$
\bar{S}_{e c}(e: c, t)=\frac{1}{2}\left[2 S\left(x_{e}: x_{c}, t\right)+S\left(z_{e}: z_{c}, t\right)\right]
$$

as the final form of our approximate formula for the total entanglement.

The introduction of the factor $1 / 2$ in the above definition is useful in the case when each of the two-dimensional subsystems are in a pure state, i.e. $S_{e c, x}(t)=S_{e c, y}(t)=S_{e c, z}(t)=0$. Then, the bipartite Schmidt theorem holds in these subspaces as $S_{e, j}(t)=S_{c, j}(t)$ (with $j=x, y, z$ ), and we obtain

$$
\bar{S}_{e c}^{(\text {pure,sep })}(t)=S_{c, x}(t)+S_{c, y}(t)+S_{c, z}(t),
$$

which is by definition the exact entanglement measure.

\subsection{Quantum entanglement in the one-dimensional model}

Now, we discuss the results of our earlier one-dimensional simulations [A1]. This model was built in complete analogy of Section 5.1, but we modeled the laser field induced entanglement dynamics of the electron and the ion-core restricted to the $z_{c} \otimes z_{e}$ subspace, described by a pure state $\Psi_{e c}^{1 \mathrm{D}}\left(z_{e}, z_{c}, t\right)$ where the $z_{e}$ and $z_{c}$ coordinates are given by the $z$ component of the coordinate transformation (1.20). A reason is, due to the large mass ratio in (5.1), we may regard the motion of the perpendicular directions $x$ and $y$ order of magnitudes slower than in the polarization direction, their contribution to the entanglement can be regarded as a constant shift. We note here, that certain simple 3D model atoms can be treated with this $1 \mathrm{D}$ approach also analytically: an example of these is the Moshinsky atom [112,113] with a single electron in an external laser field using dipole approximation.

Here, we model the hydrogen atom $(Z=1)$ using the 1D Dirac-delta potential (1.16) with the initial state (1.17). We acquire the relative part $\Psi^{1 \mathrm{D}}(z, t)$ from the $1 \mathrm{D}$ time-dependent Schrödinger equation (1.12) of Section 1.2.3. For the numerical solution of this relative wave function we implemented the Dirac-delta potential using the respective boundary condition at $z=0$ within a Crank-Nicolson scheme. For the center-of-mass wave function $\Psi_{0}^{1 \mathrm{D}}\left(z_{0}, t\right)$ we use the form of a Gaussian wave packet just like (5.1), the time evolution of which is simple 
(a)

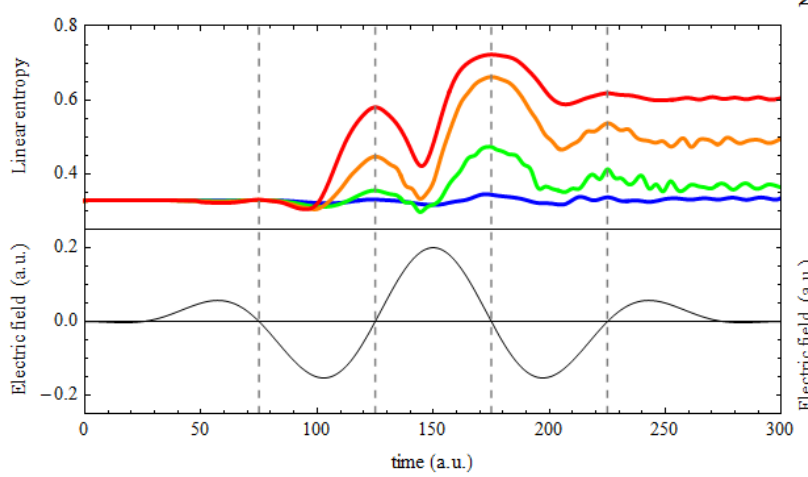

(b)

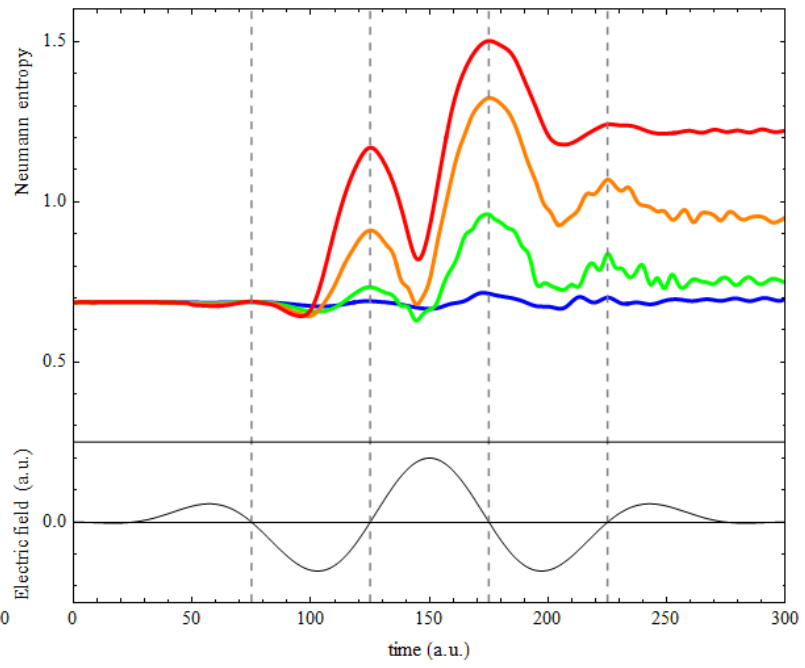

Figure 5.1: (a) Linear entropy (3.9) versus time and (b) Neumann entropy (3.8) versus time in the one-dimensional model of Section 5.3 for increasing peak values of the electric field (in atomic units). Black curves in the lower part: electric field $-\mathscr{E}_{Z}(t)$ (i.e. the electric force acting on the electron, in atomic units) of a three-cycle pulse with a sine-squared envelope (5.38). Colour curves in the upper part: Neumann entropy $S_{N}(t)$, for peak electric field $F: 0.1$ (blue), 0.15 (green), 0.2 (orange), 0.25 (red).

spreading.

In one dimension, we can use the standard bipartite entanglement quantification methods of Section 3.1 since the whole system's density matrix is pure and given by:

$$
\varrho_{e c, z}^{1 \mathrm{D}}\left(z_{e}^{\prime}, z_{e}, z_{c}^{\prime}, z_{c}, t\right)=\Psi_{e c}^{1 \mathrm{D}}\left(z_{e}^{\prime}, z_{c}^{\prime}, t\right)^{*} \Psi_{e c}^{1 \mathrm{D}}\left(z_{e}, z_{c}, t\right) .
$$

We calculate the reduced density matrix $\varrho_{c, z}^{1 \mathrm{D}}\left(z_{c}^{\prime}, z_{c}, t\right)$ by partial tracing over $z_{e}$ straightforwardly, and calculate the $S_{L}(t)$ linear entropy and $S_{N}(t)$ von Neumann entropy from this density matrix which quantify the quantum entanglement in 1D (numerically) exactly. In one dimension, the entropies $S_{c, z}(t)$ and $S\left(z_{c}: z_{e}, t\right)$ coincide with $S_{N}(t)$, therefore they are also exact entanglement measures.

The actual results using this model are presented in Fig. 5.1 (a) and (b) using a laser pulse of the form (5.38). These show the emergence of oscillations in the linear entropy and in the Neumann entropy, respectively, during the strong-field process. There is a strong correlation between the shape of the laser pulse and the oscillations of the quantum entanglement, most importantly, the local maxima of the quantum entanglement coincide with the zero crossings of the laser electric field. The oscillations become more pronounced and the final degree of entanglement also increases with higher peak values of the electric field. We also note the similarity of the $S_{N}(t)$ and $S_{L}(t)$ curves corresponding to the same case.

The results of the model discussed here also gain more relevance if we compare them to the results of a $3 \mathrm{D}$ atom. 


\subsection{Results and discussion}

In the following, we present our results regarding the three-dimensional strong-field process. First, in Section 5.4.2, we present the system's dynamical properties using two time-dependent integrated quantities. Then, in Section 4.3.2 we discuss the roles and time dependence of the entropies we introduced in this chapter. In Section 5.4.4 and Section 5.4.5 we investigate the dependence of system's entanglement dynamics on the parameters of the external field.

\subsubsection{Simulation parameters}

In our simulations, we expose the hydrogen atom to a linearly polarized few-cycle laser pulse with a sin-squared envelope function. The corresponding time-dependent electric field has nonzero values only in the interval $0 \leq t \leq 3 T$ according to the formula:

$$
\mathscr{E}_{z}(t)=F \cdot \sin ^{2}\left(\frac{\pi t}{3 T}\right) \cos \left(\frac{2 \pi t}{T}+\mathrm{CEP} \cdot \pi\right)
$$

where $T$ is the period of carrier wave, $F$ is the strength of the electric field, CEP $\cdot \pi$ is the carrierenvelope phase. We keep the wavelength of the laser field through parameter $T$ the same in all of the simulations: we set $T=100$ which corresponds to a $\sim 725 \mathrm{~nm}$ near infrared carrier wave. Varying the parameter $F$ and separately the parameter CEP, we can investigate the dynamics of the system with the emphasis on quantum entanglement. From Fig. 5.2 on, the vertical dashed lines denote the zero crossings of the respective $\mathscr{E}_{z}(t)$ electric field.

The simulation of the time evolution starts from the ground state of the relative Hamiltonian which was found by imaginary time propagation $[70,90,91]$ having the energy $\varepsilon_{0} \approx-0.49972$. Other parameters used in the numerical simulations of the relative wave function are $(i)$ discretization parameters $\Delta z=\Delta \rho=0.2$, and $\Delta t=0.01$ for the fourth-order splitting formula of (2.34), (ii) the total simulated time is 330 atomic time units, (iii) the simulation box size is varied with parameter $F$. The dimensions of the latter are $z_{\min }=-500, z_{\max }=500, \rho_{\max }=300$ for $F=0.1$. For the evaluation of the partial derivatives $z, \rho$ we use fourth-order finite differences, and for the evaluation of the integrals we also use a discrete sum approximation (2.24). After this, we perform the reduced density matrix based calculations at each atomic time unit.

\subsubsection{Dynamics}

We begin our analysis of the dynamics discussing the time dependence of the $g(t)$ ground state population loss (1.33) of the relative wave function, which is shown in Fig. 5.2 (a) for several values of $F$. We can see sudden increases of the ground state population loss that are happening at the local extrema of the electric field, more and more clearly as $F$ increases. As we have already discussed in Section 1.4, we make distinction between the tunneling ionization regime and the over-the-barrier ionization regime, regarding the dynamics dependent on $F$ : starting from the former, we see from Fig. 5.2 (a) that even for $F=0.06$, (just below the over-thebarrier threshold) the total ground state population loss is small (0.02) which implies small amount of ionization in the tunneling regime. At $F=0.10$ we have already a significant total 
(a)

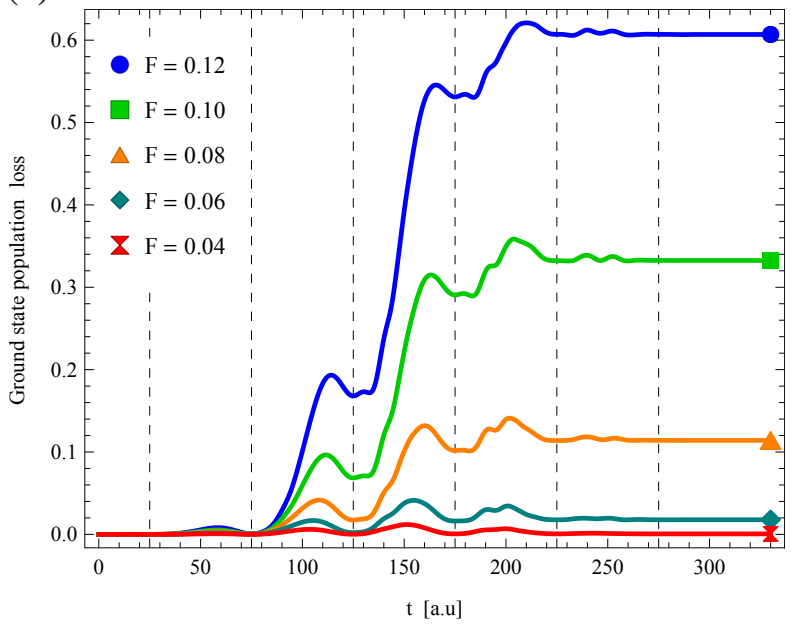

(b)

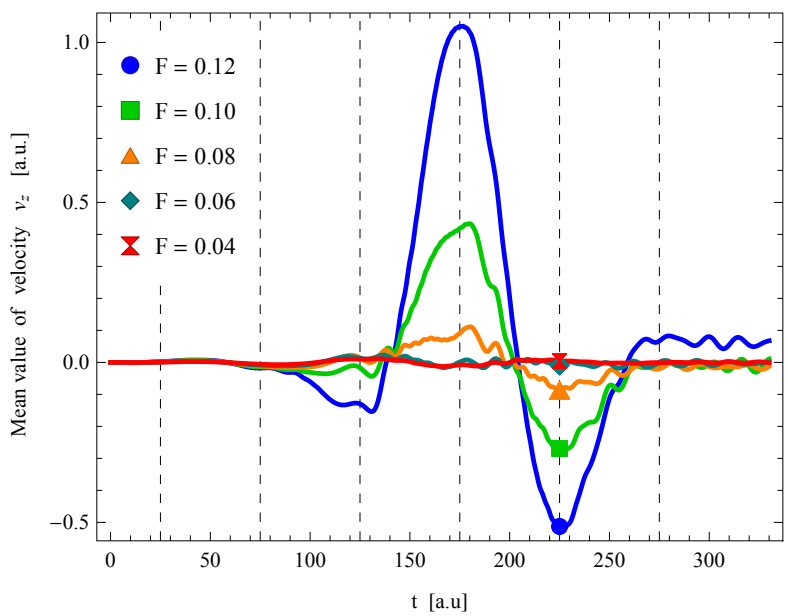

Figure 5.2: (a) Time dependence of the ground state population loss $g(t)$, and (b) time dependence of the mean velocity $\left\langle v_{z}\right\rangle(t)$ for the indicated values of the parameter $F$, with CEP $=0$. The vertical dashed lines denote the zero crossings of the electric field. (They have the same meaning on each of the figures.)

ground state population loss (0.33) with prominent over-the-barrier ionization. At the highest $F$ shown $(F=0.12)$, the electric field increasingly dominates the Coulomb force, and it almost doubles the total ground state population loss (0.61) and ionization.

From Fig. 5.2 (b), we can inspect how the results translate to an averaged classical motion, using the mean velocity component $\left\langle v_{z}\right\rangle(t)$ from the formula (1.31). In the tunneling regime (with $F=0.06$ or below), $\left\langle v_{z}\right\rangle(t)$ only slightly changes with time and has oscillating component, which implies that the relative wave function is oscillating near the origin. For amplitudes sufficiently above the over-the-barrier ionization threshold $(F=0.1)$, the velocity somewhat correlates with the quiver motion of the classical free electron moving under the influence of the oscillating electric field (5.38). For example, $\left\langle v_{z}\right\rangle(t)$ has local extrema near the zero crossings of the electric field like within this classical picture. With increasing $F$, the correlation of $\left\langle v_{z}\right\rangle(t)$ and this "free" classical motion becomes more clear, signaling the increase of importance of the ionized waves. After the laser pulse ends, the ground state population loss stops as expected, and $\left\langle v_{z}\right\rangle(t)$ appears to oscillate near a constant mean value which is more remarkable with higher $F$. (This latter value can be nonzero, which contradicts the mentioned classical picture.)

\subsubsection{Time dependence of quantum entropies}

Now we start to analyze the time-dependent dynamics of the quantum entanglement of these ionization processes.

We begin the discussion of the various quantum entropies in the direction parallel to the laser polarization axis $(z)$, then in the direction transverse to this polarization axis ( $x$ or $y)$ and in the last paragraphs in this section, we conclude with the discussion of the total electron - ion-core entanglement approximated by our method. For this task, we set the electric field parameter to be $F=0.1$ which means an intermediate, over-the-barrier ionization range, and we choose the 
(a)

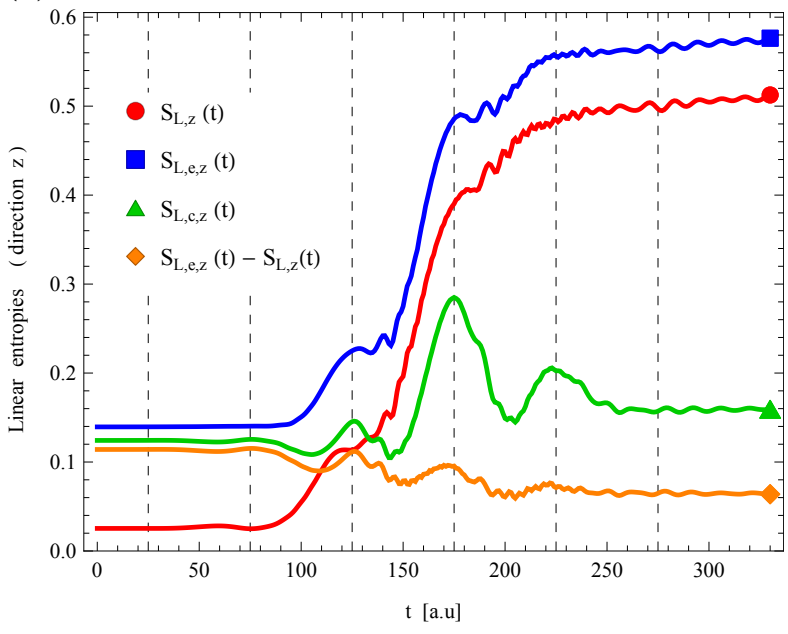

(b)

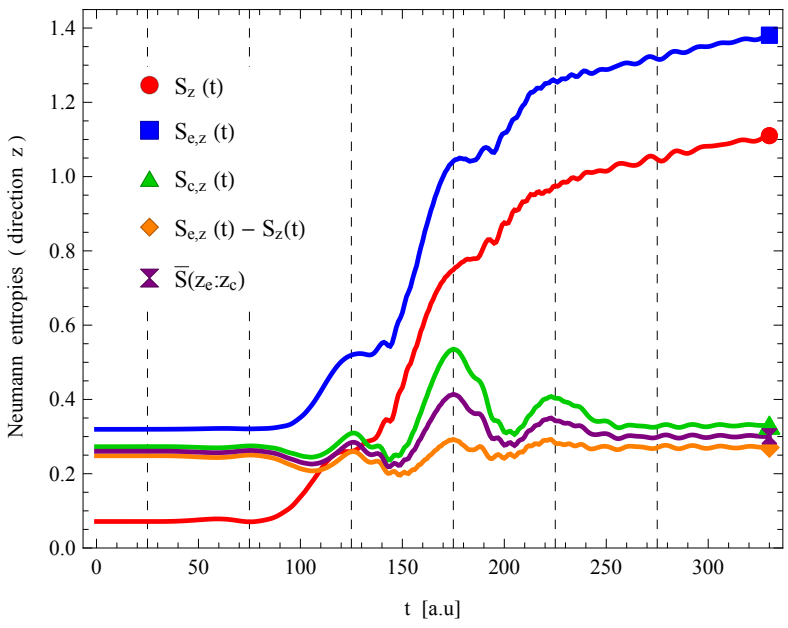

Figure 5.3: (a) Comparison of the time dependence of various linear entropies in direction $z$ using $F=0.1, \mathrm{CEP}=0$. (b) Comparison of the time dependence of various Neumann entropies in direction $z$ using $F=0.1, \mathrm{CEP}=0$. The synchronous changes in $S_{c, z}(t)$, in $S_{e, z}(t)-S_{z}(t)$, and in $\bar{S}\left(z_{e}: z_{c}, t\right)$ signal that they are related to a common source of correlation, which is primarily the quantum entanglement between $z_{e}$ and $z_{c}$, as evidenced by the high value of the negative conditional entropy $S_{e, z}(t)-S_{z}(t)$.

carrier-envelope phase to be $\mathrm{CEP}=0$.

First, we discuss the linear entropies of the reduced density matrices $\varrho_{z}, \varrho_{c, z}$ and $\varrho_{e, z}$. We use the notation $S_{L, z}(t), S_{L, c, z}(t), S_{L, e, z}(t)$ and we plot them in Fig. 5.3 (a) to provide them as a comparison to the Neumann entropies $S_{z}(t), S_{c, z}(t), S_{e, z}(t)$, which are shown in Fig. 5.3 (b), with the same parameters. Although these linear entropies compare fairly well to the respective Neumann entropies, the orange line in Fig. 5.3 (a) shows that the quantity $S_{L, e, z}(t)-S_{L, z}(t)$ gives false prediction as a negative conditional entropy, therefore we use only the Neumann entropies, as we have already stated earlier.

The time dependence of Neumann entropies corresponding to the direction $z$ are shown in Fig. 5.3 (b). We see that $S_{z}(t)$ and $S_{e, z}(t)$ share the main features but $S_{c, z}(t)$ has a different behavior. First, let us say some words about the time dependence of the Neumann entropy $S_{z}(t)$ (plotted with red line). Overall, this spatial entropy of the "spatial entanglement" between $z$ and $\rho$ has major increase during the process due to the ionization: it starts from a rather small value of 0.07 and and has a large permanent increase during the process (to the value 1.11). This entropy also continues to grow slowly but steadily after the laser pulse ended, i.e. due to the mixing effect of the Coulomb potential only. It has sudden increases in time near the peaks of the laser pulse, however, with about 10 atomic time units of delays, with the biggest jump occurring near the central peak. If we compare this plot with the corresponding curve of Fig. 5.2 (b) we clearly see that the timings of these increases synchronize with the increases of $\left|\left\langle v_{z}\right\rangle(t)\right|$. Regarding the small starting value of $S_{z}(t)$, the initial state of $\exp (-\mu r)$ is almost separable in $z$ and $\rho$, in accordance with the low value of this entropy: particularly, the dominating eigenvalue of $\varrho_{z}$ at $t=0$ is $\lambda_{1}^{(z)}=0.9872$. This clearly shows that the Neumann entropies, in general, take into account the other much smaller eigenvalues in a more pronounced way.

In Fig. 5.3 (b) we show the Neumann entropies $S_{e, z}(t)$ and $S_{c, z}(t)$, and the negative condi- 
tional entropy $S_{e, z}(t)-S_{z}(t)$, with blue, green and orange lines, respectively, in order to attempt to answer the question, how the ion-core - electron correlation works in the directional $z$ subsystem. We stated in Section 5.2, that the coordinate transformation (5.10) creates a special type of correlation. Therefore, we should be able to acquire (at least partially) the correlation information contained in $S_{c, z}(t)$ from the Neumann entropy $S_{e, z}(t)$. As it is clearly shown by Fig. 5.3 (b), the majority of the time-dependent features of $S_{e, z}(t)$ seem to be inherited from the Neumann entropy of the reduced density matrix $\varrho_{z}(t)$ (for example, the sudden increases related to the ionization), they are only shifted to higher values. However, if we carefully inspect the curve of $S_{e, z}(t)-S_{z}(t)$ in Fig. 5.3 (b) (in orange) we can easily observe that its main features (like its correlation with the laser pulse) are very similar to those of $S_{c, z}(t)$. Because these quantities are close to each other, it means that in this subsystem the major correlation is quantum entanglement, as we stated earlier. Therefore, $\bar{S}\left(z_{c}: z_{e}, t\right)$, defined in (5.19), can be used as an approximate entanglement measure. We also make the observation that $S_{z}(t)$ is always upper bounded by $S_{e, z}(t)$, and the respective $z$ coordinate of the lighter electron contains more entropy than that of the heavier ion-core, as expected.

Next, we discuss the time dependence of the resulting mutual entropy $\bar{S}\left(z_{c}: z_{e}, t\right)$, which is plotted in Fig. 5.3 (b) as a purple curve. This quantity inherits its features from $S_{c, z}(t)$ and $S_{e, z}(t)-S_{z}(t)$ by construction: it starts from an intermediate value $(0.23)$, rises and falls several times during the process, contrary to $S_{z}(t)$. It stays almost constant after the laser pulse, around a value $(0.25)$ that is only slightly higher than the initial value. The time dependence of $\bar{S}\left(z_{c}: z_{e}, t\right)$ correlates better with the shape of the laser pulse, and also has much smaller peak value in the time window, than the aforementioned spatial entropy. Interestingly, the rapid changes in ionization probability during the process are not reflected by this particle-particle entanglement of the $z$ directional subspace. The changes of this mutual entropy are more correlated with the average velocity $\left\langle v_{z}\right\rangle(t)$, which we expand more in the next section.

The curves of Fig. 5.3 (b) clearly show that the classical correlations also change under the effect of the laser pulse: the gap between $S_{c, z}(t)$ and $S_{e, z}(t)-S_{z}(t)$ is dynamically increasing and decreasing, synchronously with the electric field. Even though the respective mutual entropy includes these classical effects, the also synchronous changes in $S_{c, z}(t)$ and in $S_{e, z}(t)-S_{z}(t)$ signal that the quantum entanglement behaves the same way, and the high value of the negative conditional entropy causes it to be the major correlation.

Here we ought to note that the actual related values of $S_{c, z}(t), \bar{S}\left(z_{c}: z_{e}, t\right), S_{e, z}(t)-S_{z}(t)$ are also influenced by $\Psi_{0}$, that is by the adjustable parameter $\sigma_{0}^{2}$. According to our simulations, the change of $\sigma_{0}^{2}$ does not affect the aforementioned observations of the time-dependent characteristics of these entropies. The major difference between different values of $\sigma_{0}^{2}$ is that it results in a shift of the values $\bar{S}\left(z_{c}: z_{e}, t\right)$ and it affects the already slow dispersion rate of $\Psi_{0}$.

The time dependence of the same of quantum entropies which characterize the reduced dynamics along the $x$ axis (same along $y$ ) can be seen in Fig. 5.4 (a). However, we limited the range of the time axis (to 280 atomic time units) in this case, since one of this entropy calculations is done about $O\left(N^{4}\right)$ steps instead of $O\left(N^{3}\right)$, and it also involves that much interpolation in order to do integration in Cartesian coordinates.

From Fig. 5.4 (a), we can see a familiar shape related to the spatial entropy in the form of $S_{x}(t)$, because the values of the Neumann entropy $S_{x}(t)$ mirrors that of $S_{z}(t)$, but they are not 
(a)

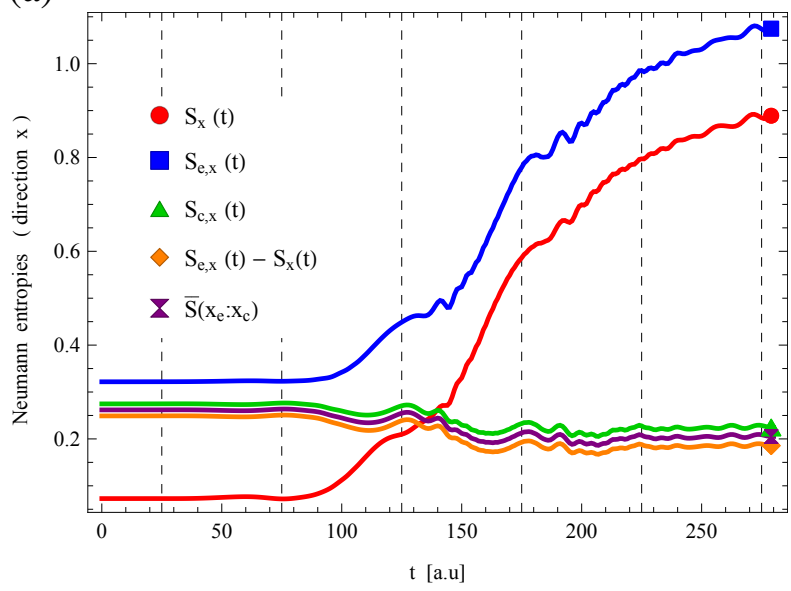

(b)

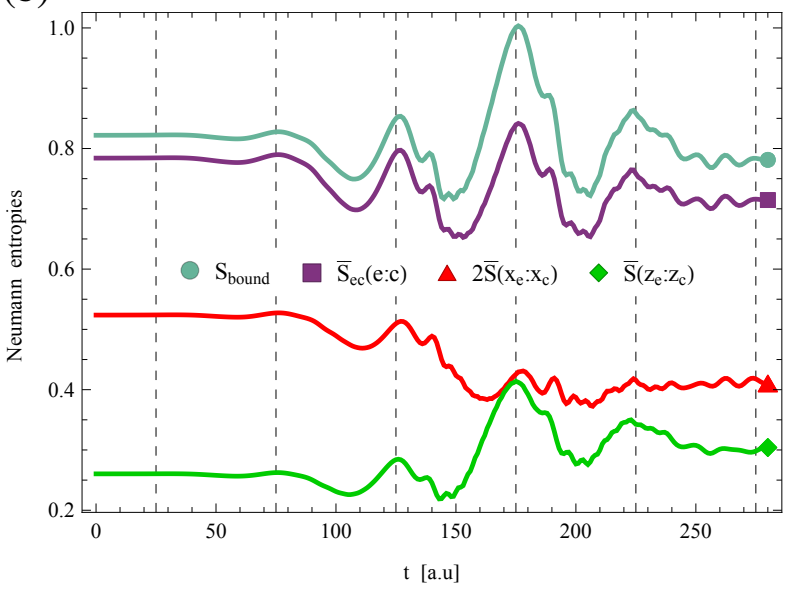

Figure 5.4: (a) The time evolution of the various Neumann entropies based on the reduced density matrices in direction $x$, using the parameters $F=0.1, \mathrm{CEP}=0$. (b) The time evolution of our electron - ion-core entanglement entropy $\bar{S}_{e c}(e: c, t)$ and the upper bound of the analytic entanglement entropy $S_{\text {bound }}(t)$, along with time evolution of the directional entropies $2 \bar{S}\left(x_{e}\right.$ : $\left.x_{c}, t\right)$ and $\bar{S}\left(z_{e}: z_{c}, t\right)$ with parameters $F=0.1, \mathrm{CEP}=0$. The time dependence of $S_{\text {bound }}(t)$ and $\bar{S}_{e c}(e: c, t)$ follow each other with substantial, and slightly increasing gap which indicates the actual importance of these curves.

the same. However, they are actually identical at $t=0$ due to the spherical symmetry of $1 \mathrm{~s}$ Coulomb state, i.e. (a single index) tripartite Schmidt decomposition [114] of the initial relative wave function exists. Then the laser pulse causes this wave function to slowly depart from this tripartite Schmidt state as $S_{x}(t)$ and $S_{z}(t)$ differ more. However, both $S_{x}(t)$ and $S_{z}(t)$ depict the time dependence of the spatial entropy adequately.

Now we turn our attention to the particle-particle correlation of the $x_{e}$ and $x_{c}$ coordinates. First, this correlation is quantum entanglement because $S_{c, x}(t)$ and its negative conditional entropy i.e. $S_{e, x}(t)-S_{x}(t)$ stay really close to each other which is only possible if $x_{e}$ and $x_{c}$ are entangled, therefore $\bar{S}\left(x_{e}: x_{c}, t\right)$ is a good entanglement measure. Now, we can also see that the entropy $\bar{S}\left(x_{e}: x_{c}, t\right)$ shares some time-dependent features with $\bar{S}\left(z_{e}: z_{c}, t\right)$, for example, its maxima are near the zero crossings of the laser pulse. Note, however, that the changes in $\bar{S}\left(x_{e}: x_{c}, t\right)$ are considerably smaller than those in $\bar{S}\left(z_{e}: z_{c}, t\right)$. It is somewhat surprising that there is an overall entanglement decrease in direction $x$, which we discuss in the next section in more detail. This decrease could be an evidence of separation between the two subsystems $x_{e}$ and $x_{c}$, as these coordinates become more uncorrelated during the physical process.

Finally, in Fig. 5.4 (b), we plot the result of our approximate formula $\bar{S}_{e c}(e: c, t)$ of the physical ion-core - electron entanglement using (5.35) with its analytic upper bound $S_{\text {bound }}(t)$ via (5.26). There, we also plot the function $\bar{S}\left(x_{e}: x_{c}, t\right)+\bar{S}\left(y_{e}: y_{c}, t\right)=2 \bar{S}\left(x_{e}: x_{c}, t\right)$ and $\bar{S}\left(z_{e}\right.$ : $\left.z_{c}, t\right)$ for the $x$ and $z$ subsystems. We see that our approximate quantification formula $\bar{S}_{e c}(e$ : $c, t)$ is clearly below $S_{\text {bound }}(t)$, with substantial, and slightly increasing gap. Also the time dependence of these follow each other, which indicates the actual importance of $\bar{S}_{e c}(e: c, t)$. It seems to be surprising that the total entanglement entropy shows a net decrease by the end of the laser pulse, which we will revisit in the next section. This is especially interesting when we take into account that other important features of the entropy $\bar{S}_{e c}(e: c, t)$ mimic those of the mutual 
(a)

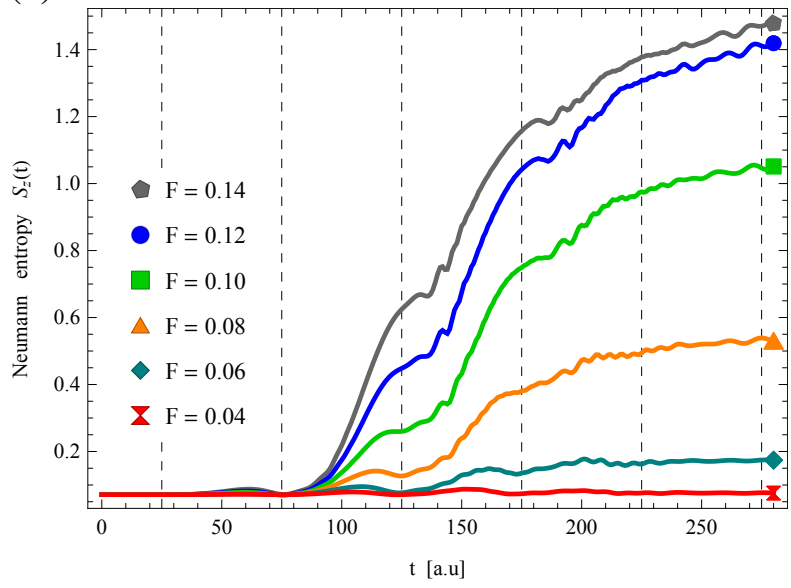

(b)

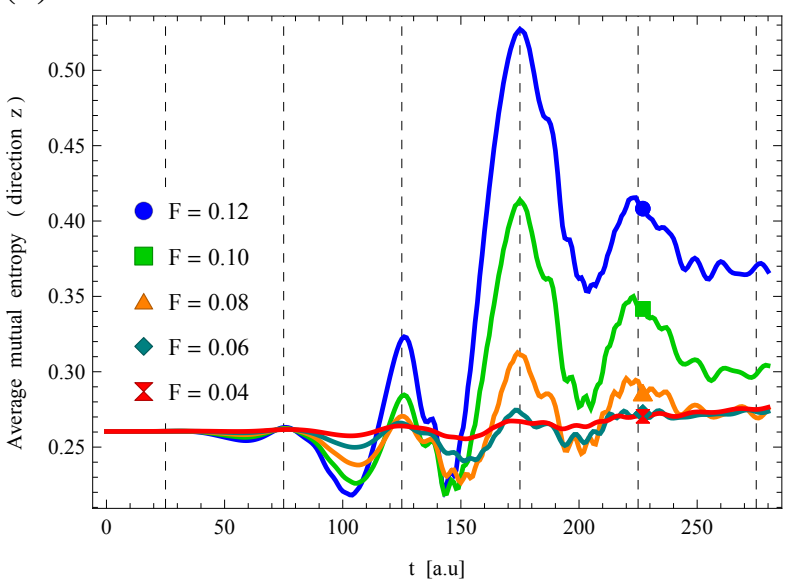

Figure 5.5: (a) The time evolution of the Neumann entropy $S_{z}(t)$ for the indicated values of the parameter $F$, with CEP $=0$. (b) The time evolution of the mutual entropy $\bar{S}\left(z_{e}: z_{c}, t\right)$ for the indicated values of the parameter $F$, with $C E P=0$. This plot is to be compared with the exact quantum entanglement entropy curves in Fig. 5.1 (b) of our 1D model simulation.

entropy $\bar{S}\left(z_{e}: z_{c}, t\right)$. In this sense we could say that the part of the relevant physics happens along the polarization axis (like the correlation with the external electric field, and the definite positions of the maxima near the zero crossings of the laser field) but the perpendicular degrees of freedom change the overall dynamics of the entanglement from increasing to decreasing.

We will further explore the dynamics of all types of entanglement presented so far in the following, while also giving more insight into the physics, by changing the external field that governs the process.

\subsubsection{Dependence on the electric-field strength}

In this section we discuss the dependence of the important entanglement entropies of Section 5.2 on the parameter $F$ i.e. on the strength of the external electric field.

In Fig. 5.5 (a) we plot the spatial entropy $S_{z}(t)$ for the relevant values of $F$. Comparing these curves with the ground state population loss of Fig. 5.2 (a) it is easy to correlate the time evolution of $S_{z}(t)$ to the probability of ionization.

Note that below the value $F=0.04$, we have only a marginal increase in $S_{z}(t)$, i.e. the relative wave function stays nearly separable in $z, \rho$ during the process. This separability quickly breaks down with increasing $F$, which is an important information regarding the applicability of the time-dependent multiconfigurational Hartree approaches [115] for the simulations of strong-field processes. It is also interesting that we have not found any specific mark of the tunneling or the over-the-barrier ionization regimes. Between $F=0.12$ and $F=0.14$, the entropy increase already slows down as a function of $F$, and one can extrapolate that the spatial nonseparability has a saturation point near $F=0.14$. We verified the existence of this maximum value with additional computations. Therefore, there is a limiting maximal value for $S_{z}(t)$ in the given time window, which already corresponds also to nearly complete ionization. The Neumann entropy $S_{z}(t)$ is not only the measure of "spatial entanglement", but it is also the total entropy of the $z$ subsystem, which has consequences regarding the interpretation of the directional mutual 
entropies.

In Fig. 5.5 (b) we plot the average mutual entropy in the directional $z$ subsystem, $\bar{S}\left(z_{e}: z_{c}, t\right)$, for the relevant range of $F$. It is easy to see that the correlation of this entropy with the shape of the laser pulse becomes more clear as we increase $F$. The values of the first minima decrease as $F$ increases, but this is reversed for the other local minima. Regarding the local maxima, they all increase with increasing $F$, the largest change occurring at the main maximum $(t=175)$. Positions of the local maxima are independent of $F$. We can observe a tunneling regime feature: the value of this entropy returns to the baseline at the end of the laser pulse. As the over-thebarrier ionization takes over (above $F=0.08$ ) the final value of the entanglement between $z_{e}$ and $z_{c}$ rises with increasing $F$.

Comparing Fig. 5.5 (b) and Fig. 5.2 (b), it is easy to recognize that the mean relative velocities $\left\langle v_{z}\right\rangle(t)$ (or alternatively, momenta) play a particularly important role regarding quantum entanglement in this direction. During one half cycle of the laser pulse, as the ion-core and the electron are moving apart, the entanglement of their respective coordinates $z_{e}$ and $z_{c}$ increases proportionally to the magnitudes of their relative velocities. The value of their entanglement decreases when deceleration occurs, and reaches its minimum value when the particles' relative motion stops. The final value of entanglement is also related to this velocity.

The results presented in Fig. 5.5 (b) are even more interesting if we compare them to the exact quantum entanglement entropy curves in Fig. 5.1 of our former 1D model simulation. Despite that the average mutual entropy $\bar{S}\left(z_{e}: z_{c}, t\right)$ includes an increasing "background" (since the composite system is always in a mixed state in the $3 \mathrm{D}$ model), the main features of the temporal dependence in Fig. 5.5 (b) and in Fig. 5.1 (b) exhibit a very good qualitative agreement: the position of the local maxima coincide with the zeros of the laser pulse, the main maximum of the entropy is roughly the double of its initial value, and the asymptotic value at the end of the simulation time scales roughly the same way to the corresponding maximum values. This agreement strongly supports our opinion that the average mutual entropy $\bar{S}\left(z_{e}: z_{c}, t\right)$ is a useful measure of quantum entanglement for the degrees of freedom along the direction of the laser polarization in the $3 \mathrm{D}$ case. The agreement also justifies the use of the delta potential in the 1D simulation, because the resulting exact ion-core - electron quantum entanglement correctly describes the corresponding entanglement dynamics of the 3D case.

Regarding the transverse direction $x$, first we note that the time dependence of $S_{x}(t)$ is very similar to that of $S_{z}(t)$ and it scales with $F$ also in an analogous way, therefore we do not plot $S_{x}(t)$. We plot $\bar{S}\left(x_{e}: x_{c}, t\right)$ in Fig. 5.6 (a) in analogy to Fig. 5.5 (b). This figure shows more clearly the striking feature that was already present in Fig. 5.4 (a): the average mutual entropy in the transverse direction decreases surprisingly strongly with increasing $F$ in the over-the-barrier ionization regime. This unexpected behavior is of purely quantum mechanical nature, contrary to direction $z$ : since $\left\langle v_{x}\right\rangle(t)=0$, there is no "classical" explanation based on the Ehrenfest kinematics. However, the positions of the local maxima $\bar{S}\left(x_{e}: x_{c}, t\right)$ are still tied to the zero crossings of the laser field. There is an importance of the tunneling regime $(F=0.0624$ and below), where the average mutual entropies $\bar{S}\left(x_{e}: x_{c}, t\right)$ and $\bar{S}\left(z_{e}: z_{c}, t\right)$ have almost the same overall behavior and show an entropy increase.

Finally, in Fig. 5.6 (b), we plot the approximate ion-core - electron entanglement $\bar{S}_{e c}(e$ : $c, t)$, defined in Eq. (5.35). Due to its construction, it inherits its features from $\bar{S}\left(z_{e}: z_{c}, t\right)$ 
(a)

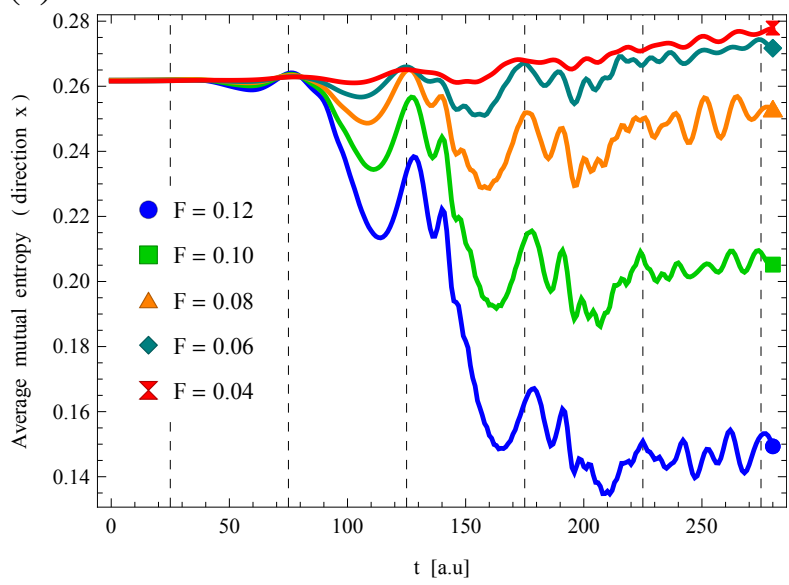

(b)

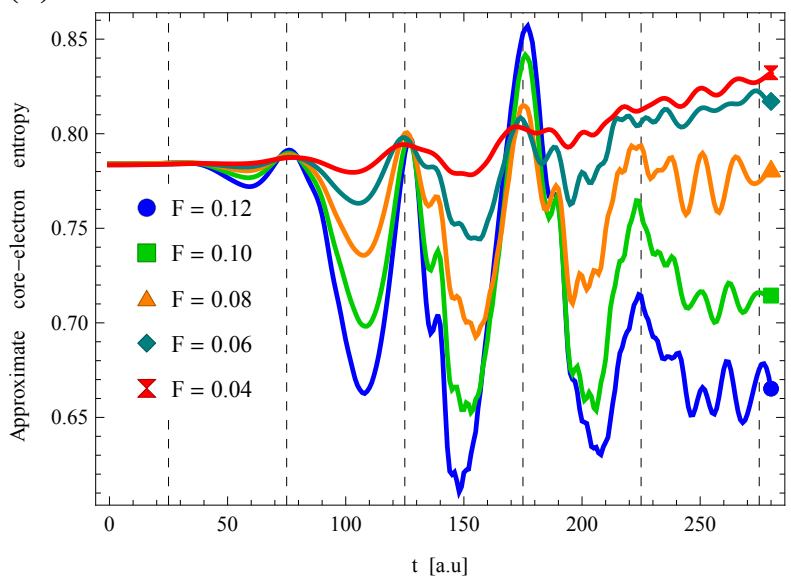

Figure 5.6: (a) The time evolution of the mutual entropy $\bar{S}\left(x_{e}: x_{c}, t\right)$ for the indicated values of the parameter $F$, with $\mathrm{CEP}=0$. (b) The time evolution of the approximate ion-core - electron entropy $\bar{S}_{e c}(e: c, t)$ for the indicated values of the parameter $F$, with CEP $=0$. Surprisingly, the entropy decrease of the transverse directions dominate the entropy increase in direction $z$, therefore this approximate ion-core - electron entanglement decreases with increasing $F$ in the over-the-barrier ionization regime.

and $\bar{S}\left(x_{e}: x_{c}, t\right)$ in the following way: if the value of $F$ ensures pure tunnel ionization, then $\bar{S}_{e c}(e: c, t)$ gains a net increase by the end of the laser pulse, otherwise the ion-core - electron entanglement decreases with increasing $F$, which is a rather surprising result. Other important features of $\bar{S}\left(z_{e}: z_{c}, t\right)$ are preserved also for $\bar{S}_{e c}(e: c, t)$ : the presence of the local maxima at the zero crossings of the laser field, the general nature of the correlations, and its link to the mean velocity.

\subsubsection{Dependence on the carrier-envelope phase}

In this section we investigate the effects of the carrier-envelope phase (CEP) on the process.

In the upper panel of Fig. 5.7 we plot the electric field of the laser pulse for our selected CEP values, with the strength of the electric field parameter set to $F=0.1$. For the sake of better comparability, we apply the following CEP-dependent transformation in time: we shift backwards the time domains in the case of nonzero CEP values such that the zero crossings of the various laser pulses coincide, as shown in the lower panel of Fig. 5.7. We plot the time dependence of some selected quantities in the following figures with this shift applied.

We plot the CEP dependence of the ground state population loss in Fig. 5.8 (a) and the mean velocity $\left\langle v_{z}\right\rangle(t)$ in Fig. 5.8 (b) using the above mentioned transformation. For each CEP value, the dynamical properties of the system stay synchronized to the local minima, maxima and zero crossings of the laser pulses. The values of the ground state population loss at the end of the laser pulse are nearly unaffected by the parameter CEP. The corresponding values of $\left\langle v_{z}\right\rangle(t)$ are only slightly affected by the CEP change.

The entanglement properties of the system inherit the above CEP related features. To show this, we plot the CEP dependence of the entropy of the "spatial entanglement" in Fig. 5.9 (a), the entropies of nonseparability in direction $z$ and in direction $x$ in Fig. 5.9 (b) and Fig. 5.10 (a) 


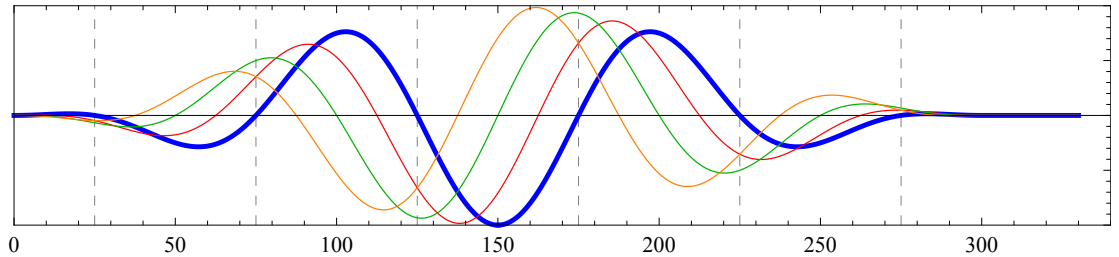

- $\mathrm{CEP}=0.00 \cdot \mathrm{CEP}=0.25 \cdot \mathrm{CEP}=0.50 \quad \mathrm{CEP}=0.75$

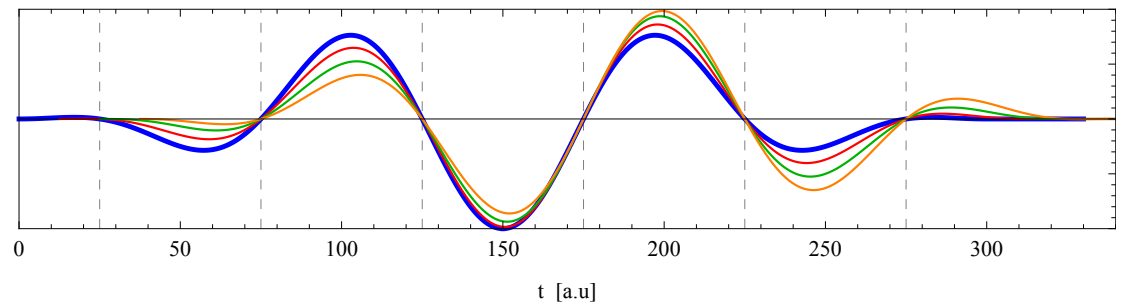

Figure 5.7: Plots of the laser pulses' electric fields $\mathscr{E}_{z}(t)$ versus time with four selected values of the parameter CEP, where the thick blue curves indicate the case of CEP $=0$. The vertical axes range from $-F$ to $F$ and represent the strength of the electric field. Plots in the upper panel are according to the formula (5.38) then we applied a CEP-dependent shift in time to make the zero crossings coincide (lower panel). We plot the time dependence of some selected quantities in Figs. 5.8 to 5.10 with this shift applied.

(a)

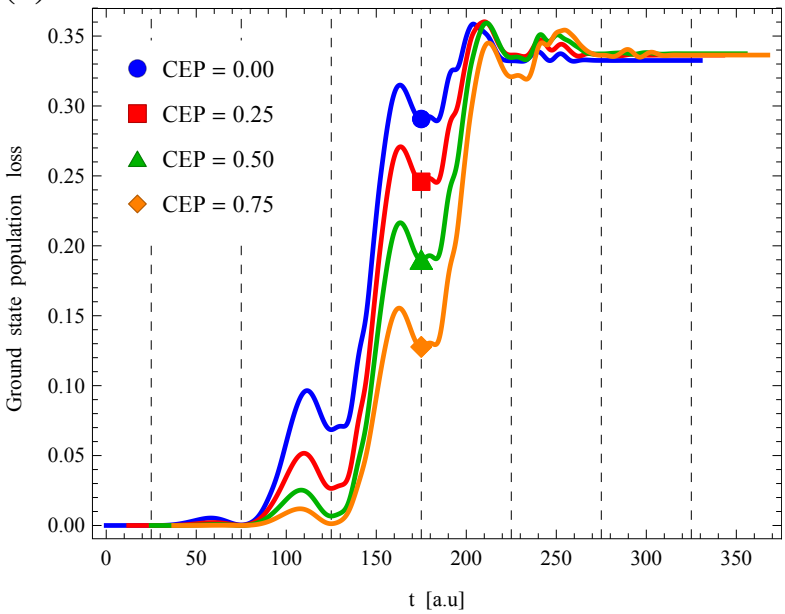

(b)

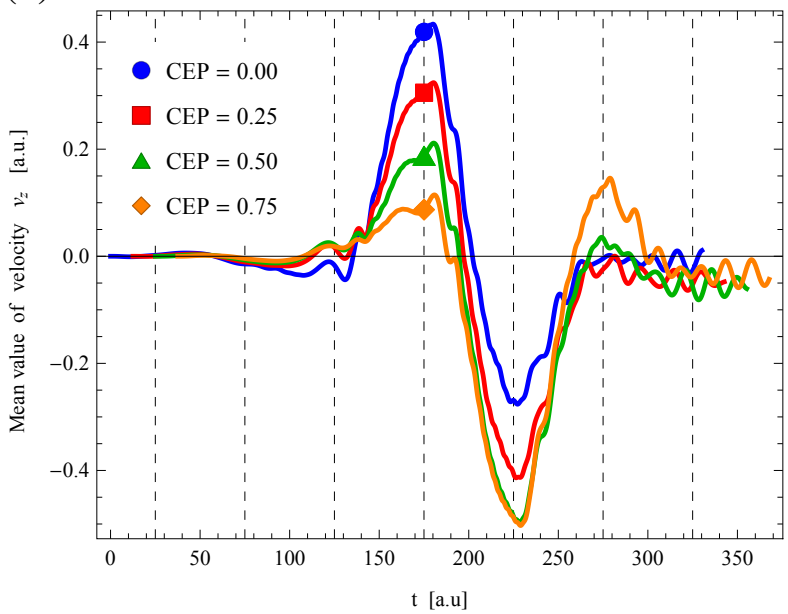

Figure 5.8: (a) Plots of the ground state population loss of the relative wave function versus time for the indicated CEP parameters, with $F=0.1$. (b) Plots of the mean velocity $\left\langle v_{z}\right\rangle(t)$ of the relative wave function versus time for the indicated CEP parameters, with $F=0.1$. 
(a)

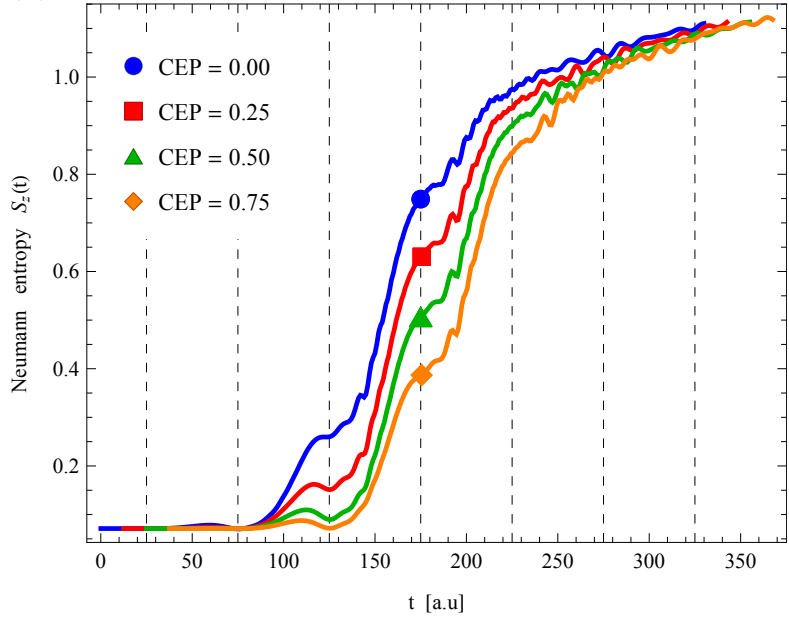

(b)

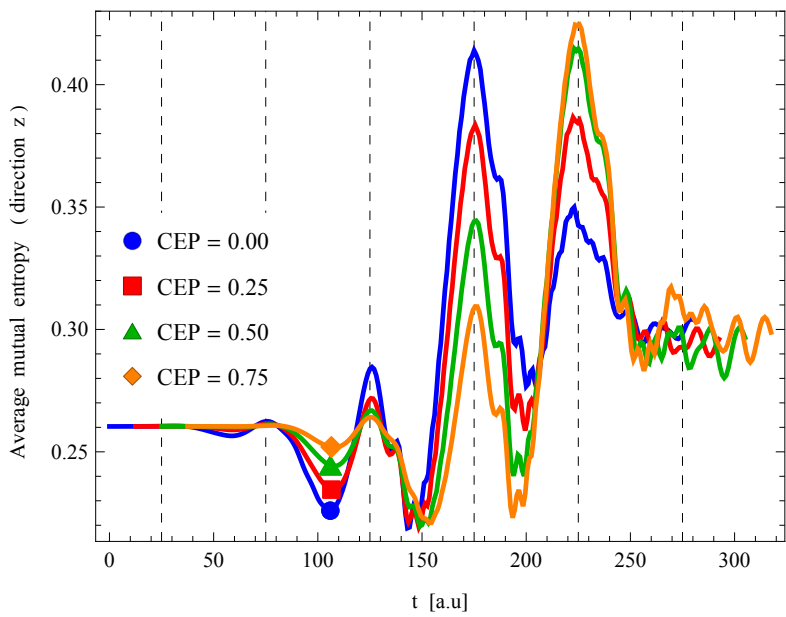

Figure 5.9: (a) Plot of the Neumann entropy $S_{z}(t)$ versus time for the indicated CEP parameters, with $F=0.1$. (b) The plot of the mutual entropy $\bar{S}\left(z_{e}: z_{c}, t\right)$ for the indicated CEP parameters, with $F=0.1$. Note that the peak at $t=175$ shrinks as the CEP increases while the peak at $t=225$ increases.

(a)

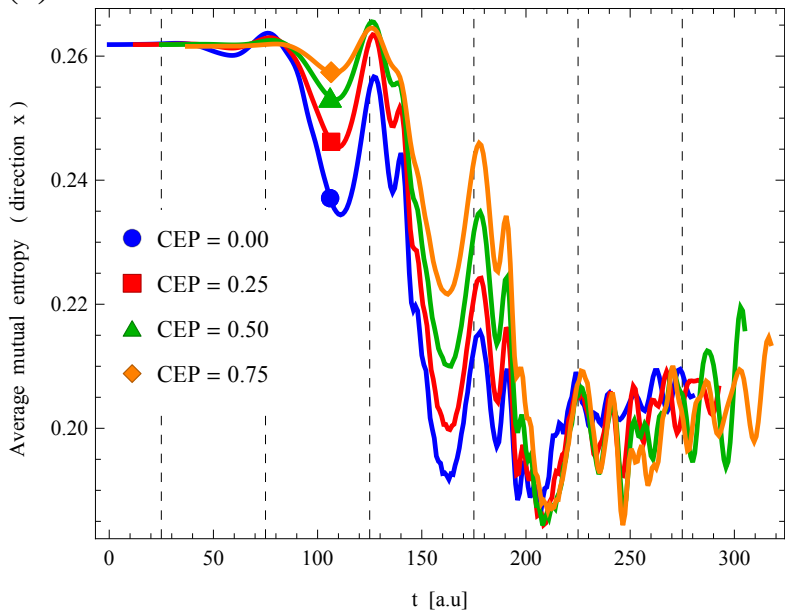

(b)

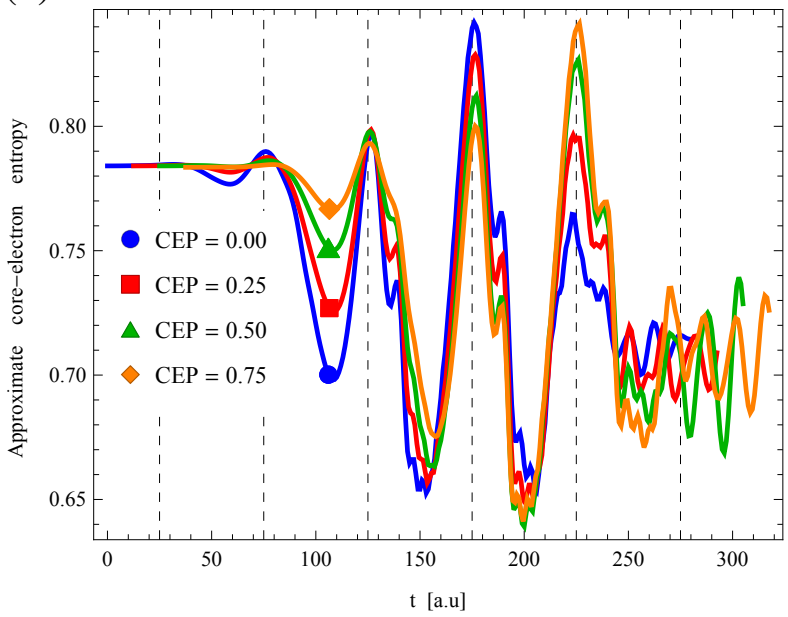

Figure 5.10: (a) The plot of the mutual entropy $\bar{S}\left(x_{e}: x_{c}, t\right)$ for the indicated values of the parameter for the indicated CEP parameters, with $F=0.1$. (b) The plot of the electron - ioncore entropy $\bar{S}_{e c}(e: c, t)$ for the indicated CEP parameters, with $F=0.1$. 
respectively, and our approximated ion-core - electron entanglement entropy in Fig. 5.10 (b). In Figs. 5.9 (b) and 5.10 (b), we can see that the local maxima still coincide with the zeros of the electric fields, independently of the CEP values, and the CEP has barely any effect on the final values. However, the actual values of the ionization, the velocities and all the entropies change considerably with respect to each other between subsequent half cycles, depending on the value of the CEP parameter. For example, in Fig. 5.9 (b) the peak at $t=175$ shrinks as CEP increases and the peak value at $t=225$ grows synchronously. We have found it interesting that the latter entropy acquires its largest value near $\mathrm{CEP}=0.75$ and not $\mathrm{CEP}=0.0$, where we have the largest value of $\mathscr{E}_{Z}(t)$. Thus, although the parameter CEP changes the subcycle dynamics of both these entropies considerably, its value does not affect our main observations about the overall time-dependent entropy dynamics.

\subsection{Summary}

In this chapter, we applied the theory of quantum entanglement and the concepts of quantum information theory to describe the time-dependent correlation properties of an electron and its parent ion-core under the influence of an external laser pulse which is strong enough to liberate the electron by tunnel or by over-the-barrier ionization.

The computation of the standard entanglement measure i.e. the Neumann entropy of either the electron or the core density matrix for this problem is numerically prohibitive in its full dimensionality, therefore we choose to partition the interacting system along the spatial directions parallel and perpendicular to the laser polarization axis, denoted by $z$ and $x$, respectively. These directionwise reduced dynamics still retain all pair correlations in $x$ and $z$. To analyze the corresponding pair correlations between the electron and the ion-core coordinates, we used several kinds of Neumann entropies that can be calculated from the one-dimensional density matrices of the system. Based on the concepts of quantum conditional entropy and quantum mutual entropy, we introduced average mutual entropies between the electron's and the ion-core's spatial position along the $x$ and $z$ directions as suitable and useful correlation measures. We constructed an approximate formula, Eq. (5.35), to quantify the total particle-particle entanglement between the electron and the ion-core, based on the directionwise mutual entropies.

We analyzed the nature of the correlations in each direction and we found that they are based on the same fundamental features of this system. For example in direction $z$, the ion-core entropy $S_{c, z}(t)$ behaves like a correlation entropy, because the ion-core density matrix is close to that of the center-of-mass which has zero entropy. The spatial entropy $S_{z}(t)$ is concentrated in the directionwise electron entropy $S_{e, z}(t)$, which also incorporates a correlation part. The resulting $S_{e, z}(t)-S_{z}(t)$, which is the negative conditional entropy of the ion-core, becomes positive and has many features in common with $S_{c, z}(t)$. In most of the simulations, these two stay really close to each other, which means that the mixed state as the function of the $z_{e}$ and $z_{c}$ coordinates shows dominantly quantum entanglement. The same is true with respect to the $x_{e}$ and $x_{c}$ coordinates. This behavior is very different from pure state entanglement, because these directional subsystems are in mixed states.

We analyzed the correlation entropy relations in each direction and we found that the zero crossings of the electric field almost coincide with their local maxima. These results in direction 
$z$ are also in a good agreement with our earlier one-dimensional simulations using the Diracdelta potential. The correlations along the $x$ and $z$ directions are very similar to each other if the process stays in the tunnel ionization regime. In the over-the-barrier ionization regime, we found entropy increase along $z$ but a surprising entropy decrease in the transverse directions which makes also the total core-electron entanglement entropy to decrease, contrary to what we expected.

We investigated the dependence of these proposed measures of entanglement dynamics on the strength and the carrier-envelope phase of the driving laser pulse. We found many features of quantum entropies that do not depend on these parameters, like the electron - ion-core entanglement has local maxima always near the zero crossings of the laser pulse. We found that while the intensity of the field governs the strength of the oscillations, the carrier-envelope phase changes the subcycle dynamics of the strong-field ionization.

Based on our simulations, we also calculated some relevant quantities that contribute to the physical picture of strong-field ionization. We found that the ground state of the simulated relative wave function is almost separable, and it remains so if the field is weak. The loss of the ground state population is a good measure of ionization, and that the net effect of the ionized waves results in a mean velocity $\left\langle v_{z}\right\rangle(t)$ which is more and more similar to the corresponding motion of a classical electron as the laser intensity increases, apart from the nonzero final velocity. 


\section{CHAPTER 6}

\section{One-dimensional density-based model potentials}

In the present chapter, we introduce and test novel one-dimensional (1D) atomic model potentials to be used in the simulations of strong-field ionization driven by a linearly polarized laser pulse. We aim to introduce atomic model potentials that quantitatively correctly describe this process, without actually performing 3D simulations. Our key idea is to require the ground state density of the 1D model to be equal to the reduced 3D ground state density, obtained by integrating over spatial coordinates perpendicular to the direction of the laser polarization. According to density functional theory [116, 117], this 1D ground state density determines the corresponding 1D model potential up to a constant, which we set by matching the ground state energies. These results are based on our publication [P3].

In Section 6.1, we derive the analytic one-dimensional formula of this density-based model potential, and we discuss any possible improvements that can be made to the 1D Coulomb-like model potentials of Section 1.2.3. Then, in Section 6.2, we introduce a novel numerical method that constructs 1D numerical model potentials through their analytical ground states, and we verify the improved numerical accuracy of this method for nondifferentiable model potentials. In Section 6.3, we compare our improved 1D model potentials and the corresponding 3D model by applying them in careful numerical simulations of strong-field ionization by a few-cycle laser pulse. Based on these results we make a conclusion about the best of these novel 1D model potentials.

\subsection{Theoretical model}

We describe the strong-field process in one dimension within the framework of Section 1.2.3 by solving the $1 \mathrm{D}$ time-dependent Schrödinger equation (1.12). In the following, we are going to derive the density-based formula for the atomic model potential $V_{0}^{1 \mathrm{D}}(z)$ within the onedimensional atomic model Hamiltonian (1.13), and based on its peculiarities we will suggest certain improvements in other known 1D model potentials. 


\subsubsection{Derivation of the 1D analytical model potential}

Our inspiration of deriving this new 1D model potential originated from the Kohn-Sham theory [118] of multielectron atoms. More specifically, the following derivation is analogous to the derivation of the Kohn-Sham potential of a helium atom with a single orbital: knowing the correct reduced (single-particle) density [117] one can invert the Schrödinger equation to determine this potential. In this way one can model the ground state of the system as accurately as possible with a single orbital. However, here we consider just single-active-electron atoms and we make the analogous reduction from the 3D electron coordinates to the $z$ coordinate of the single electron.

For developing our 1D model potential, we need the 1D reduced density of the 3D Coulomb ground state that is defined by

$$
\varrho_{z}^{100}(z)=2 \pi \int_{0}^{\infty}\left|\psi_{100}(z, \rho)\right|^{2} \rho \mathrm{d} \rho
$$

After the substitution of the ground state function (1.9) for the integrand, we can perform this integral analytically which yields the closed form

$$
\varrho_{z}^{100}(z)=\frac{\mu Z}{2}(2 Z \mu|z|+1) e^{-2 Z \mu|z|} .
$$

Now we require the $1 \mathrm{D}$ model system to have its ground state density identical with $\varrho_{z}^{100}(z)$. According to density functional theory, this $1 \mathrm{D}$ ground state density determines the corresponding $1 \mathrm{D}$ model potential $V_{0, \mathrm{M}}^{1 \mathrm{D}}(z)$ up to a constant. We can calculate this potential straightforwardly: we define the ground state of the $1 \mathrm{D}$ model atom obviously as $\psi_{0}(z)=\sqrt{\varrho_{z}^{100}(z)}$, i.e.

$$
\psi_{0}(z)=\sqrt{\frac{\mu Z}{2}} \sqrt{2 \mu Z|z|+1} e^{-\mu Z|z|}
$$

and then we invert the eigenvalue equation of $H_{0}^{1 \mathrm{D}}$ as

$$
V_{0, \mathrm{M}}^{1 \mathrm{D}}(z)=E_{0, \mathrm{M}}+\frac{1}{\psi_{0}(z)} \frac{1}{2 \mu} \frac{\partial^{2}}{\partial z^{2}} \psi_{0}(z) .
$$

After performing the differentiation we get

$$
V_{0, \mathrm{M}}^{1 \mathrm{D}}(z)=E_{0, \mathrm{M}}+\frac{2 \mu^{3} Z^{4}|z|^{2}-\mu Z^{2}}{(2 \mu Z|z|+1)^{2}}
$$

In order to determine the ground state energy, we rewrite this potential as

$$
V_{0, \mathrm{M}}^{1 \mathrm{D}}(z)=E_{0, \mathrm{M}}+\frac{\mu Z^{2}}{2} \frac{(2 \mu Z|z|+1)(2 \mu Z|z|-1)-1}{(2 \mu Z|z|+1)^{2}},
$$

and then we impose the asymptotic value

$$
\lim _{|z| \rightarrow \infty} V_{0, \mathrm{M}}^{1 \mathrm{D}}(z)=0
$$




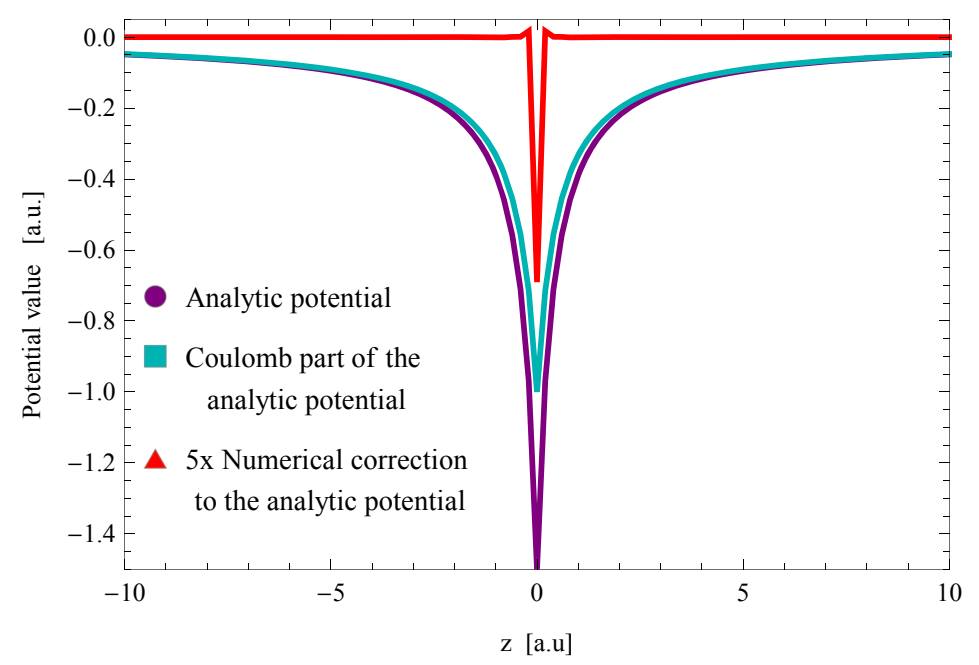

Figure 6.1: Plot of the analytic potential (6.9) (in purple) and its regularized Coulomb part (second term of (6.9), in cyan), for $Z=1, \mu=1$. We also plot the difference $\widetilde{V}_{0, \mathrm{M}}^{1 \mathrm{D}}(z)-V_{0, \mathrm{M}}^{1 \mathrm{D}}(z)$, (see the discussion in Section 6.2) calculated with for $\Delta z=0.2$, and magnified by a factor of 5 (in red). This is to illustrate the numerical correction to be introduced by Eq. (6.15).

which yields the ground state energy

$$
E_{0, \mathrm{M}}=E_{0}=-\frac{\mu Z^{2}}{2}
$$

Using this value, after some algebraic manipulations we arrive at the following instructive form of our new density-based 1D atomic model potential:

$$
V_{0, \mathrm{M}}^{1 \mathrm{D}}(z)=-\frac{1}{2 \mu} \frac{1}{2^{2}\left(|z|+\frac{1}{2 \mu \mathrm{Z}}\right)^{2}}-\frac{\frac{1}{2} Z}{|z|+\frac{1}{2 \mu Z}} .
$$

Let us make a few important notes. It is the asymptotic tail of the reduced 1D ground state density $\varrho_{z}^{100}(z)$ that determines the ground state energy $E_{0, \mathrm{M}}$ in such a nontrivial way that it is identical to the ground state energy of the 3D system, $E_{0}$. The asymptotic tail of $\varrho_{z}^{100}(z)$ also determines the regularized $1 \mathrm{D}$ Coulomb potential with effective charge $\frac{1}{2} Z$ which is the second term in (6.9). This term is dominant over the short-range first term of (6.9) not only in the asymptotic tail but also around the center at least by a factor of 2, see the corresponding curves of Fig. 6.1. The minima of both of the two terms of $V_{0, M}^{1 \mathrm{D}}(z)$ at $z=0$ decrease with increasing $Z$ or $\mu$. For $Z=1$ and $\mu=1$, the energy of its first excited state is $E_{1, \mathrm{M}}=-0.0904408$ approximately.

\subsubsection{Improved 1D model potentials}

The results of previous section, especially the somewhat surprising value of an effective charge of $\frac{1}{2} Z$, suggested by the second term of the analytical model potential (6.9), inspire us to use the $1 \mathrm{D}$ soft-core Coulomb potential and a 1D regularized Coulomb potential with accordingly modified values of their parameters. As we will see, these modifications lead indeed to improved results in strong-field simulations. 
We use $\frac{1}{2} Z$ in the nominator of the soft-core Coulomb potential (1.14), which then requires to change also the parameter $\alpha_{\mathrm{Sc}}$ in order to maintain that its ground state energy matches the 3D ground state energy. These lead us to the following formula of the improved 1D soft-core Coulomb potential with $\mu=1$ :

$$
V_{0, \mathrm{M}, \mathrm{Sc}}^{1 \mathrm{D}}(z)=-\frac{\frac{1}{2} Z}{\sqrt{z^{2}+\frac{1}{4 Z^{2}}}} \text { with } E_{0, \mathrm{M}, \mathrm{Sc}}=-\frac{Z^{2}}{2},
$$

that has the correct $\frac{1}{2} Z /|z|$ asymptotic behavior when $|z| \rightarrow \infty$. The energy of its first excited state is $E_{1, \mathrm{M}, \mathrm{Sc}}=-0.1058670$.

We also introduce the improved 1D regularized Coulomb potential from (1.18) as

$$
V_{0, \mathrm{M}, \mathrm{C}}^{1 \mathrm{D}}(z)=-\frac{\frac{1}{2} Z}{|z|+a},
$$

where the value of the parameter $a$ is determined by requiring that the ground state energy is $E_{0, \mathrm{M}, \mathrm{C}}=-\frac{\mu Z^{2}}{2}$. For $Z=1$ we set $a \approx 0.32889$ which yields $E_{0, \mathrm{M}, \mathrm{C}} \approx-0.5000007$ (for $\mu=1$ ). We note that this has been computed numerically with the spatial step size $\Delta z=0.2$.

The sophisticated numerical method to be outlined in the next section will enable us to make additional refinements regarding the 1D density-based potential, as well as the 1D Dirac-delta potential. These improvements will be explained below especially by the formulas given in Eqs. (6.15) and (6.16).

\subsection{Numerical model}

In the numerical model, we use the Crank-Nicolson method for the numerical solution of the respective 1D time-dependent Schrödinger equation (1.12) which we discussed in Section 2.1. In the following, we show that with the help of analytical eigenfunctions and by taking advantage of the discretization of the kinetic energy operator, we can construct improved numerical representations of the density-based potential (6.9) and the 1D Dirac-delta potential (1.16) using finite differences. In Sections 6.2.2 and 6.2.3 we present details about the accuracy of these numerical model potentials.

\subsubsection{Density-based numerical model potentials}

Here, we introduce the particular form of the finite difference discretization that is used for making the Hamiltonian matrix. To outline our method, we discretize the spatial coordinate with uniform steps $\Delta z$ as $z_{i}=z_{\min }+i \Delta z$, where $i \in\left[0, N_{z}\right]$ is an integer index. Then, the discretized wave function is written as $\Psi^{1 \mathrm{D}}\left(z_{i}, t_{k}\right)$. We write the discretized form of the $1 \mathrm{D}$ atomic model Hamiltonian as

$$
\widetilde{H}_{0}=\widetilde{T}_{z}+V_{0}^{1 \mathrm{D}}\left(z_{i}\right),
$$


where, based on our experiences detailed in Section 4.3.2, we use a very-high-order finite difference method [68] for the discretization of the kinetic energy operator $T_{z}$ :

$$
\begin{gathered}
\widetilde{T}_{z} \Psi\left(z_{i}, t_{k}\right)=-\frac{1}{2 \mu} \sum_{s=-5}^{5} \frac{c_{|s|}^{(5)}}{\Delta z^{2}} \Psi^{1 \mathrm{D}}\left(z_{i}+s \Delta z, t_{k}\right), \quad \text { where } \\
c_{0}=-\frac{5269}{1800}, c_{1}=\frac{5}{3}, c_{2}=-\frac{5}{21}, c_{3}=\frac{5}{126}, c_{4}=-\frac{5}{1008}, c_{5}=\frac{1}{3105} .
\end{gathered}
$$

(We also outline the general method of calculating finite difference coefficients in Appendix A.) This formula is accurate up to $\Delta z^{10}$ for smooth functions (it is also limited by the Fourier representation). Then, the discrete Hamiltonian becomes a banded 11-diagonal matrix which operates on the column vector of the discretized wave function in coordinate representation. Regarding the use of the atomic model potential in numerical simulations, this is the most important step since it defines the numerical eigensystem of the atom.

When using the potential (6.10) the method described above can be applied without further complications. In the case of our density-based model potential a refinement is necessary as (6.9) is not differentiable in the origin, just as the true 3D Coulomb potential. Therefore the ground state and energy of the discrete Hamiltonian (6.12) with $V_{0, \mathrm{M}}^{1 \mathrm{D}}\left(z_{i}\right)$ is accurate only up to $\Delta z^{2}$. This is the reason why its ground state density does not equal $\varrho_{z}^{100}\left(z_{i}\right)$ accurately enough, unless $\Delta z$ is extremely small. We propose to avoid this inaccuracy in the following way: instead of $V_{0, \mathrm{M}}^{1 \mathrm{D}}\left(z_{i}\right)$, we use its following discretized form in the computations:

$$
\widetilde{V}_{0, \mathrm{M}}^{1 \mathrm{D}}\left(z_{i}\right)=E_{0}-\frac{1}{\psi_{0}\left(z_{i}\right)} \widetilde{T}_{z} \psi_{0}\left(z_{i}\right)
$$

This definition of $\widetilde{V}_{0, \mathrm{M}}^{1 \mathrm{D}}\left(z_{i}\right)$ ensures that the discretized ground state vector $\psi_{0}\left(z_{i}\right)$ is the eigenvector of (6.12) with $\widetilde{V}_{0, \mathrm{M}}^{1 \mathrm{D}}\left(z_{i}\right)$ and the corresponding energy is $\widetilde{E}_{0, \mathrm{M}}=E_{0}$, numerically exactly. The energy of the corresponding first excited state (with $\Delta z=0.2$ ) is $\widetilde{E}_{1, \mathrm{M}}=-0.0904385$, which is close enough to $E_{1, \mathrm{M}}$. We have plotted the difference $\widetilde{V}_{0, \mathrm{M}}^{1 \mathrm{D}}\left(z_{i}\right)-V_{0, \mathrm{M}}^{1 \mathrm{D}}\left(z_{i}\right)$ in Fig. 6.1, magnified by a factor of 5 .

The discretized form of the analytical model potential, $\widetilde{V}_{0, \mathrm{M}}^{1 \mathrm{D}}\left(z_{i}\right)$ suggests also a modified discretization of the 1D Dirac-delta potential that we introduce as

$$
\widetilde{V}_{0, \mathrm{DD}}^{1 \mathrm{D}}\left(z_{i}\right)=E_{0, \mathrm{DD}}-\frac{1}{\psi_{0, \mathrm{DD}}\left(z_{i}\right)} \widetilde{T}_{z} \psi_{0, \mathrm{DD}}\left(z_{i}\right)
$$

using the corresponding exact ground state $\psi_{0, \mathrm{DD}}\left(z_{i}\right)$ and energy $E_{0, \mathrm{DD}}$. This is a finite discretized potential which eliminates any singular feature from the corresponding Hamiltonian matrix.

As we will show it in the subsequent sections, such definitions enable consistent and accurate simulations with high-order finite differences. 
(a)

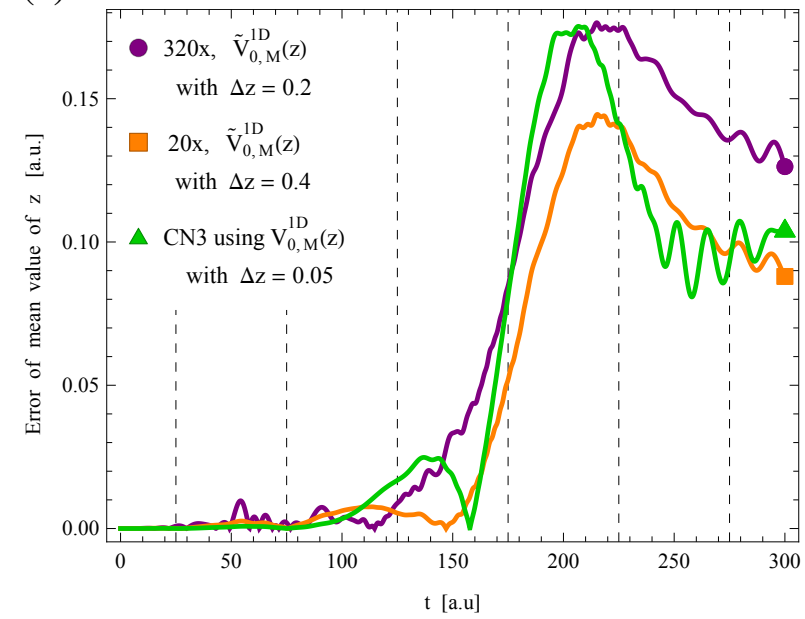

(b)

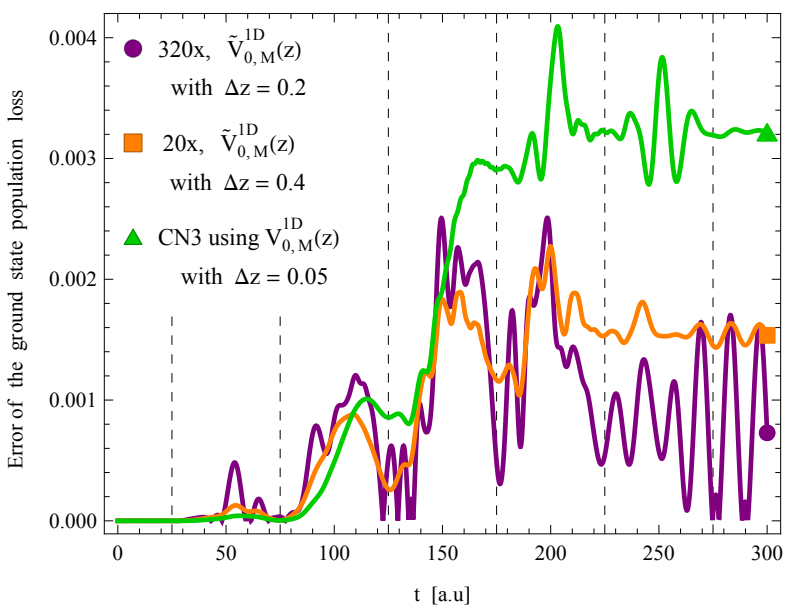

Figure 6.2: (a) Time dependence of the numerical mean value errors $\left|\langle z\rangle(t)-\langle z\rangle_{\text {ref }}(t)\right|$ and (b) ground state population loss errors $\left|g(t)-g_{\text {ref }}(t)\right|$ using different realizations of the densitybased model potentials, under the influence of the same external field (6.17) with $F=0.1$, $N_{\text {Cycle }}=3$. We plotted in purple and orange the results using the potential $\widetilde{V}_{0, \mathrm{M}}^{1 \mathrm{D}}$ from numerical inversion formula (6.15) with $\Delta z=0.2$, and $\Delta z=0.4$, respectively. Note that the values of these two curves are magnified by a factor of 320 and 20 as indicated. For comparison, we plotted in green the results directly using the analytic formula (6.9) as the atomic potential in an usual Crank-Nicolson solution. The vertical dashed lines denote the zero crossings of the electric field. (They have the same meaning on all the subsequent figures of this chapter.)

\subsubsection{Test results: density-based 1D model potential}

We stated previously that the numerical construction (6.15) yields the exact numerical eigensystem of that potential, but that does not give us the whole picture about how numerically accurate the construction really is. If we look at the eigenenergy of its respective first excited state calculated with $\Delta z=0.2$ we see that it is $4-5$ digit accurate, but that alone does not determine the usefulness in strong-field simulations. To get the whole picture, we performed numerical simulations using the atomic potential (6.15) and a 3-cycle laser pulse of form (6.17) with $F=0.1$ with different $\Delta z$ parameters. We subtracted from them the results of a very accurate reference numerical solution using the analytical potential (6.9) with $\Delta z=0.0001$, which gave us information about the (approximate) numerical errors of the construction.

The results can be seen on Fig. 6.2, where we plotted the errors of mean values $\langle z\rangle(t)$ and the ground state population losses $g(t)$ compared to the reference versus time. We can see that if we decrease the spatial step $\Delta z$ of the inversion (6.15) from 0.4 (orange) to 0.2 (purple) the error decreases approximately by a factor of 16, in the case of both $\langle z\rangle(t)$ and $g(t)$. We verified this using also other integrated quantities: we can clearly assert that the numerical inversion (6.15) is around $\Delta z^{4}$ accurate i.e. it shows high-order accuracy (required that the kinetic energy operator is also at least $\Delta z^{4}$ accurate). To illustrate what this means, we also plotted the results obtained by the usual 3-point finite difference Crank-Nicolson method (CN3) using the analytical potential (6.9) as the atomic potential, which are known to be $\Delta z^{2}$ accurate. We briefly note that we tested the direct use of (6.9) with our 11-point finite difference scheme but it was not any better, also $\Delta z^{2}$ accurate (since the potential is not differentiable), so we only 
(a)

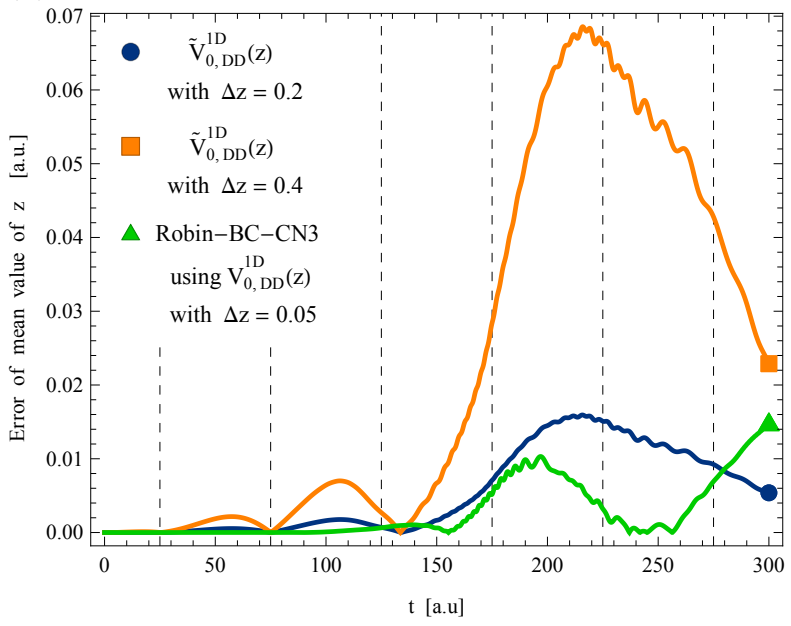

(b)

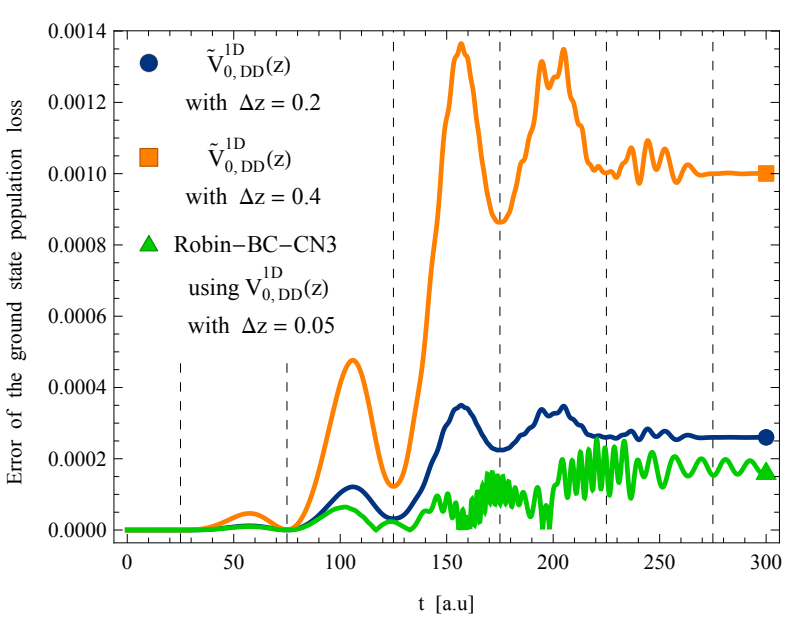

Figure 6.3: (a) Time dependence of the numerical mean value errors $\left|\langle z\rangle(t)-\langle z\rangle_{\text {ref }}(t)\right|$ and (b) ground state population loss errors $\left|g(t)-g_{\text {ref }}(t)\right|$ using different implementations of the 1D Dirac-delta model potential, under the influence of the same external field (6.17) with $F=0.1$, $N_{\text {Cycle }}=3$. We plotted in dark blue and orange the results using the potential $\widetilde{V}_{0, \mathrm{DD}}^{1 \mathrm{D}}$ from numerical inversion formula (6.16) with $\Delta z=0.2$, and $\Delta z=0.4$, respectively. For comparison, we plotted in green the results using the implementation in [A1] that uses the proper Robin boundary condition to represent the singularity of (1.16).

plotted the results of the $\mathrm{CN} 3$ scheme in Fig. 6.2 with green lines. The accuracy of this method using $\Delta z=0.2$ is around 320 times better than the direct use of the analytical nondifferentiable potential with $\Delta z=0.05$. So in other words it requires $2^{6}$ more spatial gridpoints $(\Delta z \approx 0.003)$ than the numerical inversion.

Therefore, using the formula (6.15) to numerically represent (nonsingular) model potentials is very efficient and shows high-order convergence.

\subsubsection{Test results: 1D Dirac-delta potential}

In the following, we discuss the accuracy tests of the numerically constructed potential (6.16) using strong-field simulations with the same 3-cycle laser pulse of form (6.17) with $F=0.1$ and different $\Delta z$ parameters. For comparison we use a properly implemented method from [A1] that uses the proper Robin boundary condition at $z=0$, which overrides the Crank-Nicolson equations at that grid point. Its results are at least $\Delta z^{2}$ accurate. We calculate the errors of the mean values $\langle z\rangle(t)$ and the ground state population losses $g(t)$ compared to a very accurate reference solution obtained by this correct method (using $\Delta z=0.001$ ).

We can see the results on Fig. 6.3. Surprisingly, we can observe that the errors of (6.16) with $\Delta z=0.2$ are actually not far from the errors of results obtained by the $\Delta z^{2}$ accurate proper method at $\Delta z=0.05$. If we decrease the $\Delta z$ step from 0.4 (orange) to 0.2 (dark blue) we see a factor 4 error decrease: we can conclude that the nonsingular construction (6.16) is actually correct numerical representation, and converges with $\Delta z^{2}$ even for the singular delta potential. It is also of importance because of the following: we can run simulations with singular potentials using nonsingular Hamiltonians, and the point of singularity is not have to be on the spatial 
grid, it can even move. It has even more interesting consequences in $2 \mathrm{D}$ or more, since there is no reason not to work with the true singular Coulomb potentials.

In conclusion, it is a valid choice to define potentials using numerical inversion from their ground state. It provides a consistent and accurate method with high-order finite differences to represent our (6.9) nonsingular and nondifferentiable atomic potential in 1D, and even achieve $\Delta z^{4}$ convergence. The method is robust enough to provide $\Delta z^{2}$ convergence for the case of the singular 1D delta potential using (6.16).

\subsection{Results and comparison of the 1D and 3D calculations}

In this section, we present the results of various strong-field simulations based on the 1D model potentials introduced in Sections 1.2.3 and 6.1. We selected the mean value $\langle z\rangle(t)$ and its standard deviation $\sigma_{z}(t)$, the mean velocity $\left\langle v_{z}\right\rangle(t)$, and the ground state population loss $g(t)$ to characterize the dynamics resulting from the solutions of the 1D time-dependent Schrödinger equation (1.12) and the solutions of 3D Schrödinger equation (1.2.2) in cylindrical coordinates as a reference. For this latter, we use our hybrid splitting method of Chapter 4. We also investigate the relation between the resulting various dipole power spectra $p(f)$, which is one of the most important quantities for high-order harmonic generation $[7,10]$ and attosecond pulses.

\subsubsection{Simulation procedure}

In these simulations, we also use a linearly polarized few-cycle laser pulse with a sine-squared envelope function similar to (5.38). The corresponding time-dependent electric field has nonzero values only in the interval $0 \leq t \leq N_{\text {Cycle }} T$ according to the formula:

$$
\mathscr{E}_{z}(t)=F \cdot \sin ^{2}\left(\frac{\pi t}{N_{\mathrm{Cycle}} T}\right) \cos \left(\frac{2 \pi t}{T}\right),
$$

where $T$ is the period of the carrier wave, $F$ is the peak electric field strength and $N_{\text {Cycle }}$ is the number of cycles under the envelope function. Unless otherwise stated, we set $N_{\text {Cycle }}=3$ and $T=100$, the latter corresponds to a ca. $725 \mathrm{~nm}$ near-infrared carrier wavelength. We use the same function $\mathscr{E}_{z}(t)$ both in the $1 \mathrm{D}$ and 3D simulations.

We consider hydrogen in most of the simulations, i.e. we use $Z=1$ and $\mu=1$ if not otherwise stated explicitly. We set typically $\Delta z=0.2$ and $\Delta t=0.01$ since these are sufficient for the numerical errors to be within line thickness. We use box boundary conditions and we set the size of the box to be sufficiently large so that the reflexions are kept below $10^{-8}$ atomic units.

The 3D reference results (i.e. the simulation results of the true 3D Schrödinger equation (1.6)) are plotted in Figs. 6.4 to 6.11 in blue and are labeled "3D-reference". The 1D simulation results and their respective colors are plotted as follows: our density-based model potential from numerical inversion (6.15) in purple, our improved soft-core Coulomb potential (6.10) in gold, our improved regularized Coulomb potential (6.11) in red, the conventional soft-core Coulomb potential (1.14) in green and the discretized Dirac-delta potential (6.16) in dark blue. 
(a)

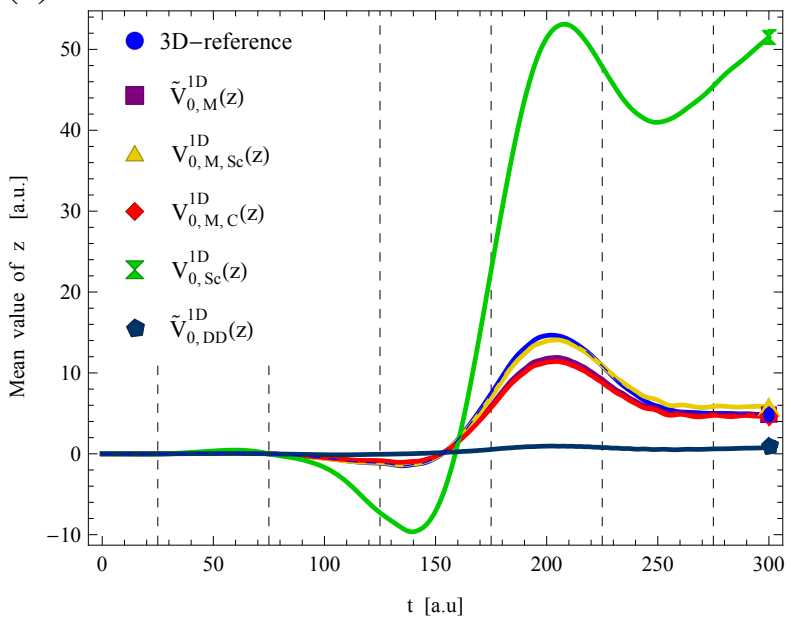

(b)

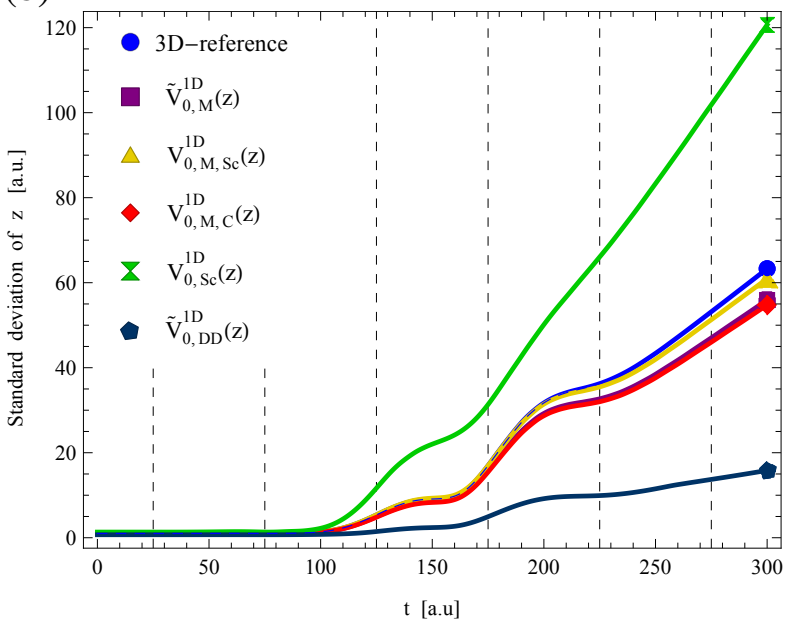

Figure 6.4: (a) Time dependence of the mean values $\langle z\rangle(t)$ and (b) the standard deviations $\sigma_{z}(t)$ using different 1D model potentials, under the influence of the same external field with $F=0.1, N_{\text {Cycle }}=3$ and $T=100$. Results of the corresponding 3D simulation are plotted in blue.

\subsubsection{Low frequency response}

First, we discuss the results of a moderately strong laser pulse having a peak electric field value of $F=0.1$. We plot the corresponding time-dependent mean values $\langle z\rangle(t)$ (the magnitude of which equals the dipole moment in atomic units) and their standard deviations $\sigma_{z}(t)$ in Fig. 6.4, the time-dependent mean velocities $\left\langle v_{z}\right\rangle(t)$ and the ground state population losses $g(t)$ in Fig. 6.5 for all the 1D model systems listed above. These curves justify that the simulation results obtained with our density-based model potential and the improved model potentials are already quantitatively comparable to the $3 \mathrm{D}$ results, i.e. these model potentials capture the essence of the $3 \mathrm{D}$ process. This fact is in strong contrast with the poor results of the conventional 1D softcore and 1D Dirac-delta potentials, which is caused mainly by their too weak and too strong binding force, respectively.

The graphs of the improved soft-core Coulomb potential are clearly at the closest to the 3D reference in most of these cases, i.e. this potential provides the quantitatively best model of the $3 \mathrm{D}$ case, despite that its ground state density is not the exact reduced density of the 3D case. The results of our numerical density-based model potential are somewhat less close to the 3D reference. Although these simulations start from the exact reduced density of the 3D case, the electron is somewhat stronger bound to the ion-core than optimal. The results obtained using the improved regularized Coulomb potential are very close to those of the density-based model potential, but the former potential is even somewhat stronger than needed.

In a typical strong-field simulation, the ground state population loss $g(t)$ is close to the probability of ionization. Due to the presence of the transverse degrees of freedom in $3 \mathrm{D}$, it is then reasonable that the $g(t)$ values are somewhat larger in a 3D simulation than in $1 \mathrm{D}$. Note that the $g(t)$ curves of the 1D simulations follow very well the 3D reference curve in accordance with this.

The lack of the transverse degrees of freedom affects the $\left\langle v_{z}\right\rangle(t)$ curves of the 1D simula- 
(a)

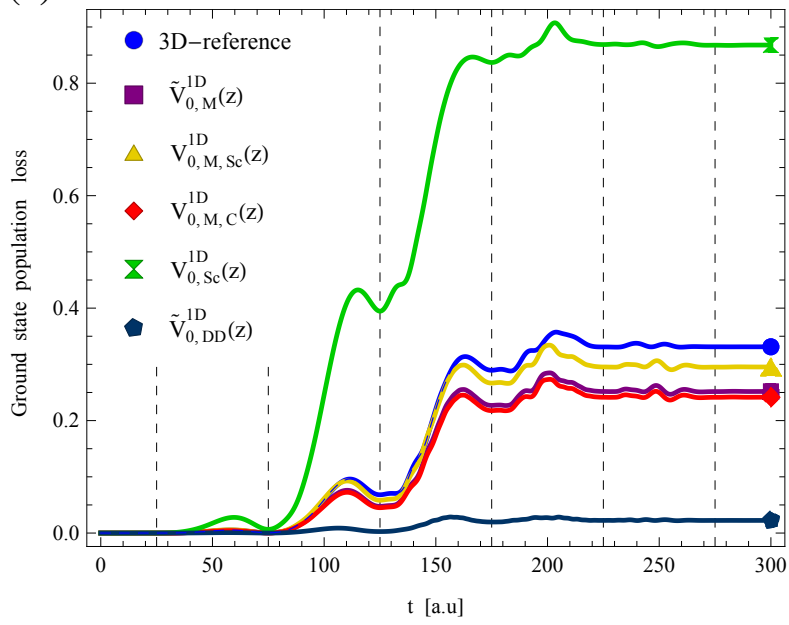

(b)

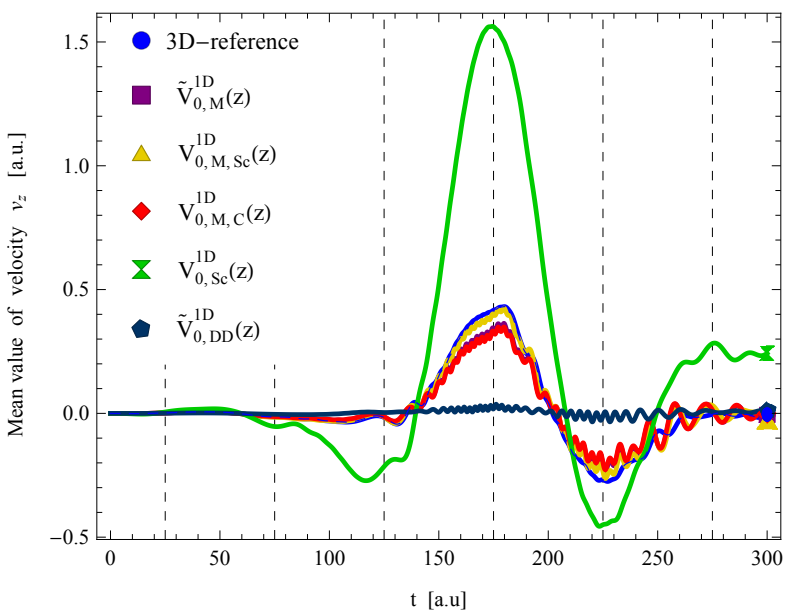

Figure 6.5: (a) Time dependence of the ground state population loss $g(t)$ and (b) the mean velocities $\left\langle v_{z}\right\rangle(t)$ using different 1D model potentials, under the influence of the same external field with $F=0.1, N_{\text {Cycle }}=3$ and $T=100$.

tions in a different way: these exhibit the high-frequency oscillations with larger amplitude than the 3D reference curve. This can be explained by taking into account that rescattering on the ion-core is a much stronger factor in $1 \mathrm{D}$, and that the integration over the transverse directions decreases the effect of the 3D density oscillations on the reduced mean values. We will analyze this in more detail in the next section.

In order to demonstrate the capabilities of these novel 1D model potentials, we selected the time-dependent dipole moment $\langle z\rangle(t)$ to present the results of 4 different scenarios in Fig. 6.6 and Fig. 6.7. Since the curves corresponding to the density-based model potential are very close to those corresponding to the improved regularized 1D Coulomb potential, we do not plot the $\langle z\rangle(t)$ of this latter potential in all of our figures.

In Fig. 6.6 (a) we plot our simulation results for hydrogen, now with a weaker field of $F=0.05$ which is in the tunnel ionization regime of hydrogen, while Fig. 6.6 (b) corresponds to a stronger field of $F=0.15$. Both of these figures clearly show that the improved 1D softcore Coulomb potential provides the best results. Note that the change of $F$ in the above range results in more than 2 orders of magnitude change in the peak value of $\langle z\rangle(t)$.

Fig. 6.7 (a) shows the results for a Ne atom driven by a field of $F=0.15$. Here we model the $3 \mathrm{D}$ neon atom in the single-active-electron approximation [19] simply by setting the Coulombcharge $Z_{\mathrm{Ne}}^{(\mathrm{SAE})}=1.25929$ as in (1.11) in order to match the ionization potential to the experimental value. (For the improved regularized Coulomb potential $V_{0, \mathrm{M}, \mathrm{C}}^{1 \mathrm{D}}$ we set $a_{\mathrm{Ne}}^{(\mathrm{SAE})} \approx 0.26707525$ which yields $E_{0, \mathrm{M}, \mathrm{C}} \approx-0.792905$.)

The accuracy of these 1D results is somewhat lower around the peak and in the last halfperiod of the laser pulse than in the case of hydrogen, and the improved soft-core Coulomb potential performs considerably better in overall than the two other model potentials. By changing the Coulomb charge $Z$ within a reasonable range in order to model different noble gas atoms, we have obtained similarly accurate results.

Fig. 6.7 (b) shows $\langle z\rangle(t)$ for a hydrogen atom, now driven by a longer laser pulse of shorter carrier wavelength, corresponding to the parameters $T=80, F=0.1$, and $N_{\text {Cycle }}=6$. The $1 \mathrm{D}$ 
(a)

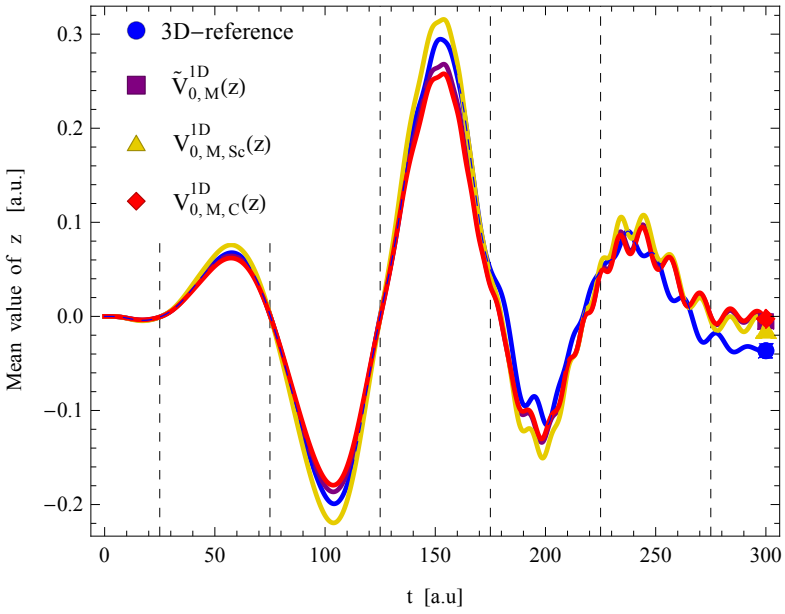

(b)

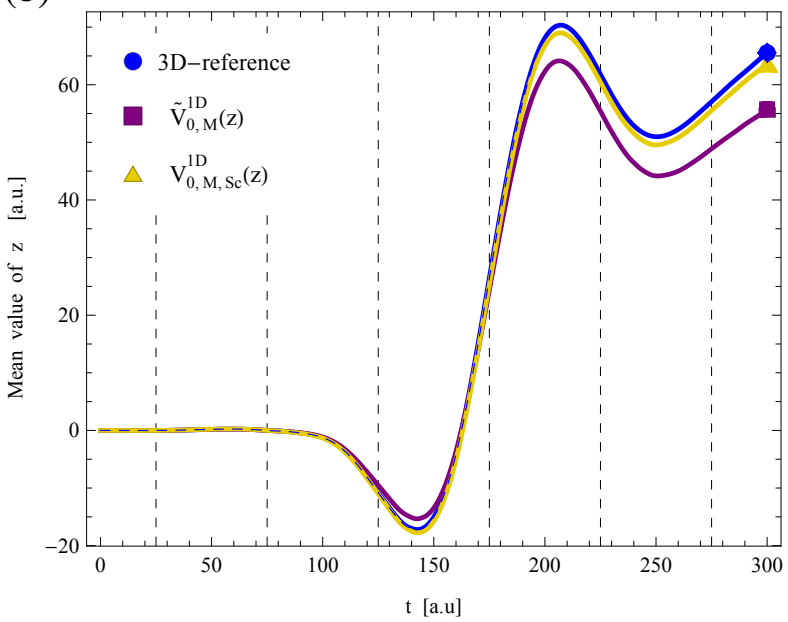

Figure 6.6: Time dependence of mean values $\langle z\rangle(t)$ using different 1D model potentials, under the influence of the external field with $F=0.05$ (a) and $F=0.15$ (b), $N_{\text {Cycle }}=3$ and $T=100$.

(a)

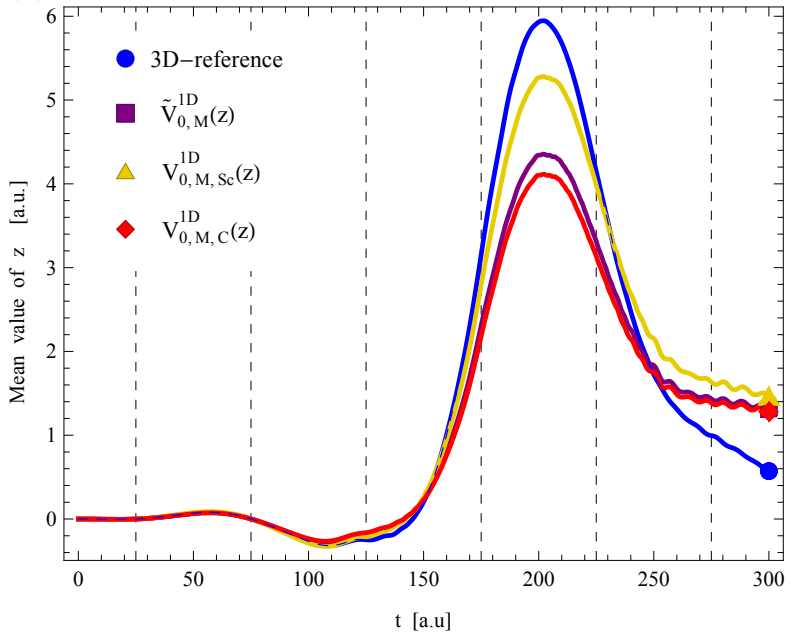

(b)

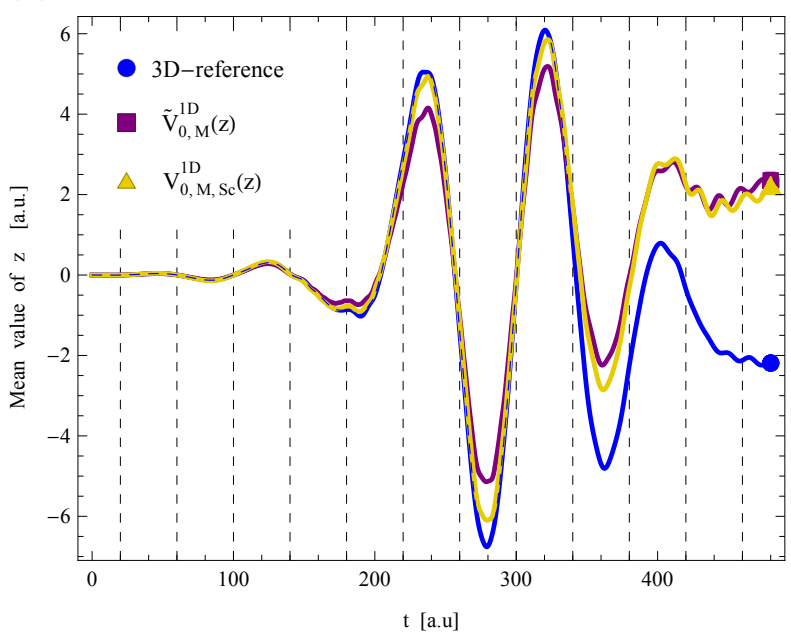

Figure 6.7: Time dependence of mean values $\langle z\rangle(t)$ using different 1D model potentials. Panel (a): single-active-electron model of a neon atom with $Z_{\mathrm{Ne}}^{(\mathrm{SAE})}=1.25929$, driven by the external field with $T=100, F=0.15, N_{\text {Cycle }}=3$. Panel (b): hydrogen with $Z=1$ driven by the external field with $T=80, F=0.1, N_{\text {Cycle }}=6$. 
(a)

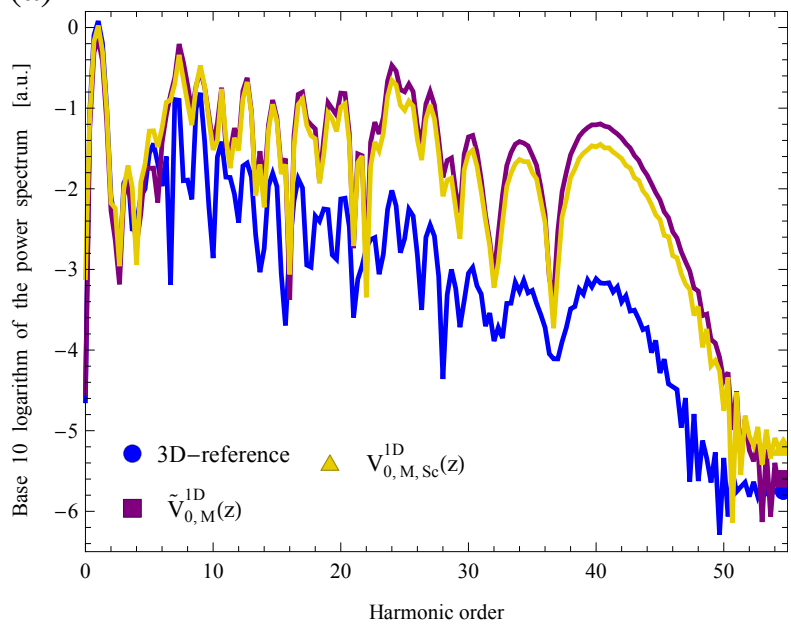

(b)

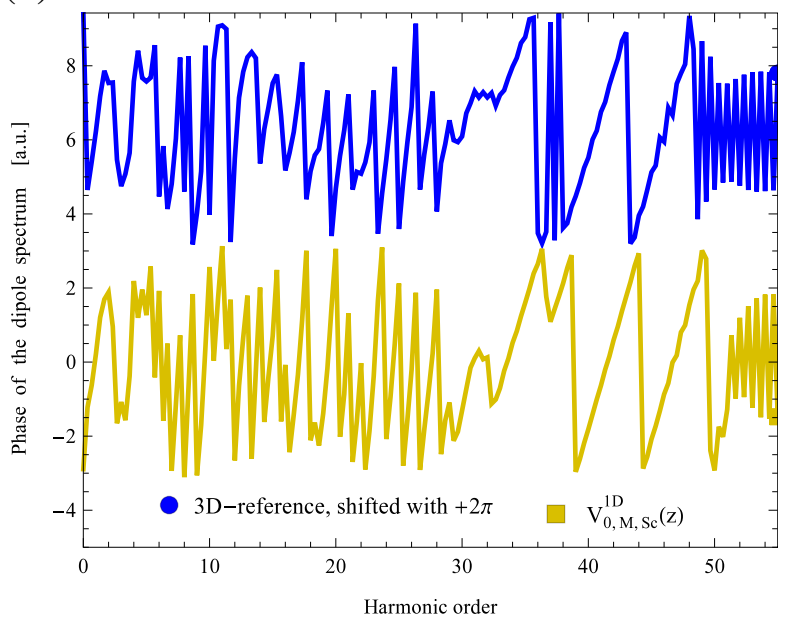

Figure 6.8: Panel (a): Logarithmic plot of the power spectra vs. the harmonic order, i.e. $p\left(n f_{1}\right)$ (where $f_{1}=1 / T=0.01$ a.u. is the fundamental frequency). Panel (b): Phase of the dipole acceleration spectra vs. the harmonic order (up shifted by $2 \pi$ for the $3 \mathrm{D}$ case). We plot the results for the density-based 1D model potential (purple) and for the improved soft-core Coulomb potential (gold) in comparison with the 3D reference (blue). The parameters $F=0.1$, $N_{\text {Cycle }}=3, T=100$ and $Z=1$ are the same as for Figs. 6.4 and 6.5.

model potentials work similarly accurately for this longer laser pulse as in the case presented in Fig. 6.4 (b), until the recollisions with the ion-core gradually decrease the match between the $1 \mathrm{D}$ and $3 \mathrm{D}$ cases in the last 2 periods of the pulse.

Our density-based 1D model potential and both of the improved 1D model potentials exhibit an impressive improvement in the accuracy of the low-frequency response of typical strongfield processes, in contrast to the two conventional model potentials. These results are even more convincing if we take into account that $\langle z\rangle(t), \sigma_{z}(t)$ and $g(t)$ are very sensitive to almost any change in the physical parameter values.

\subsubsection{High-order harmonic spectra}

In strong-field physics, the accurate computation of the high-order harmonic spectrum is especially important, because this represents the highly nonlinear atomic response to the strong-field excitation, with well-known characteristic features $[6-8,10]$.

In Fig. 6.8 (a), we plot the power spectrum $p(f)$ of the dipole acceleration (see Eq. (1.35)) for the parameters corresponding to Figs. 6.4 and 6.5 .

In agreement with the previous section, the power spectra obtained using the 1D model potentials agree very well with the $3 \mathrm{D}$ reference simulation result up to the 5 th harmonic. For higher frequencies, the 1D spectra gradually deviate and give 1-2 orders of magnitude larger values than the $3 \mathrm{D}$ reference values. The explanation given for the oscillations of the $\left\langle v_{z}\right\rangle(t)$ curves in Fig. 6.5 (b) applies also here: 1D simulations exaggerate the effect of the ion-core, mainly via rescattering, while the effect of the $3 \mathrm{D}$ density oscillations weakens in the reduced mean values obtained from the $3 \mathrm{D}$ simulation.

However, the structure of the spectra in Fig. 6.8 (a) is remarkably similar and the match 
(a)

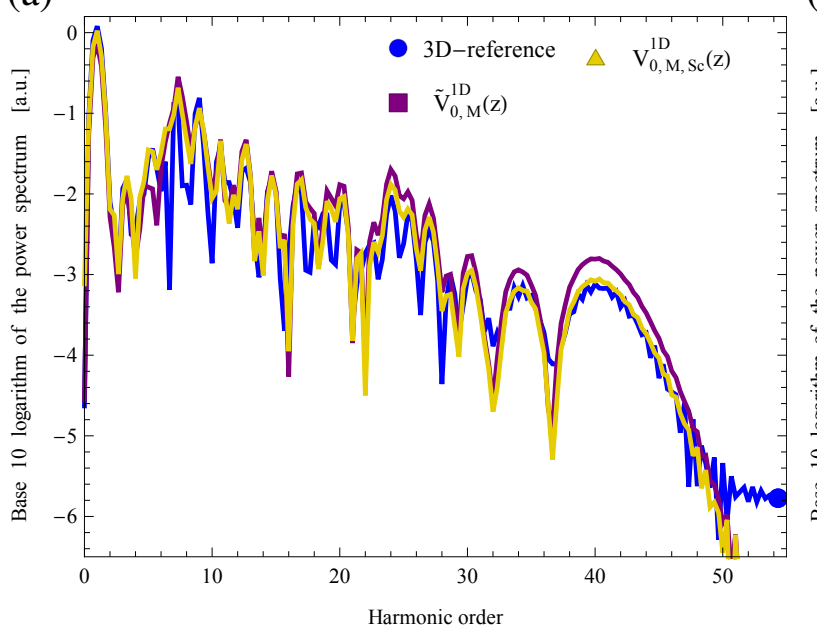

(b)

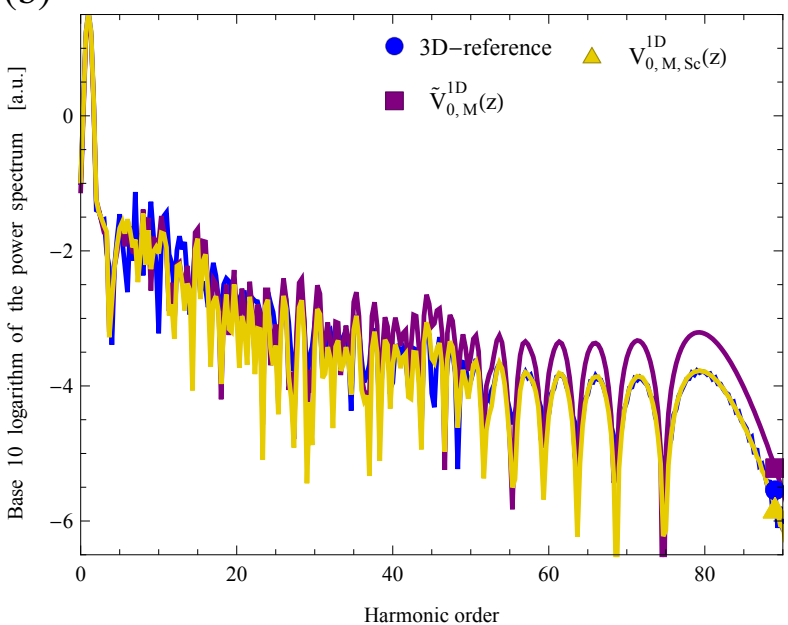

Figure 6.9: Logarithmic plots of the scaled power spectra $p\left(n f_{0}\right) / s\left(n f_{0}\right)$ using the model systems of Fig. 6.8 with $F=0.10$ (a), $F=0.15$ (b), in comparison with the 3D reference (blue).

(a)

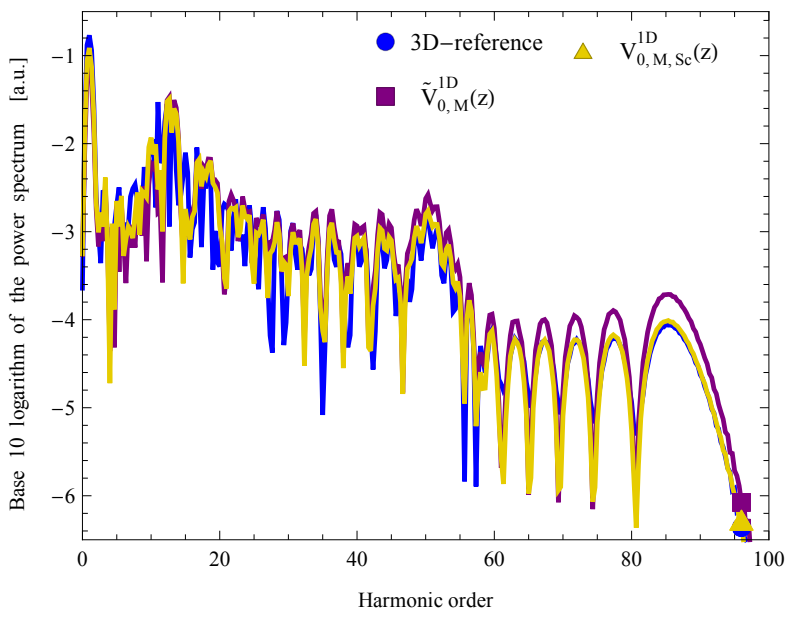

(b)

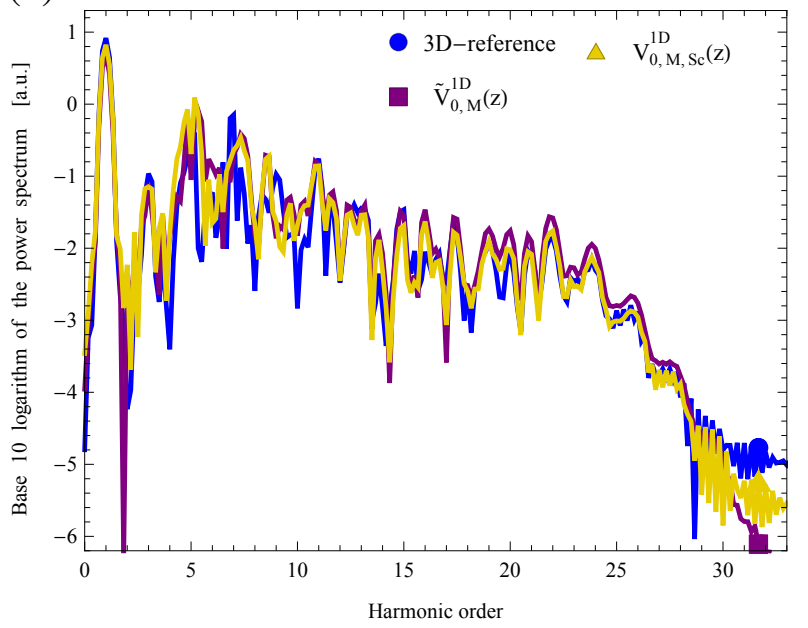

Figure 6.10: Logarithmic plots of the scaled power spectra $p\left(n f_{0}\right) / s\left(n f_{0}\right)$ obtained using the density-based 1D model potential (purple) and the improved soft-core Coulomb potential (gold), in comparison with the 3D reference (blue). Panel (a): single-active-electron model of a neon atom with $Z_{\mathrm{Ne}}^{(\mathrm{SAE})}=1.25929$ driven by the external field with $T=100, F=0.15$, $N_{\text {Cycle }}=3$. Panel (b): hydrogen with $Z=1$, driven by the external field with $T=80, F=0.1$, $N_{\text {Cycle }}=6$. 
of the spectral phase, shown in Fig. 6.8 (b), is very good, especially in the higher frequency range, which is of fundamental importance for isolated attosecond pulses. These inspired us to create a scaling function which transforms the spectra obtained with the 1D simulation to fit the 3D reference spectrum as correctly as possible. Since the improved soft-core Coulomb potential (6.10) gives the best low-frequency results, we focus only on this model potential in the following.

Examination of the ratio of the magnitudes of the $1 \mathrm{D}$ power spectrum to the $3 \mathrm{D}$ power spectrum in our simulations with different parameters revealed that the scaling function

$$
s(f)=\min \left(1+0.03(100 f-1)^{2}, 1+|100 f-1|\right)
$$

transforms the magnitude of the power spectra obtained using the improved 1D soft-core Coulomb potential to properly fit the corresponding 3D power spectra. In Fig. 6.9 (a) we plot the scaled 1D power spectrum $p(f) / s(f)$ which gives a very good match between the $3 \mathrm{D}$ and $1 \mathrm{D}$ results in the case of the improved soft-core Coulomb potential. (Here and in the following figures we plot the scaled power spectrum of the density-based 1D model potential for completeness only.) In Fig. 6.9 (b) and Fig. 6.10 (a) and (b) we present this comparison for three other scenarios, corresponding to the parameters of Fig. 6.6 (b) and Fig. 6.7 (a) and (b), respectively. These plots clearly show that the scaling function (6.18) works very well also in these cases.

\subsubsection{Electron - ion-core quantum entanglement along direction $\mathrm{z}$}

In the following, we would like to further demonstrate that our improved model potentials quantitatively capture the dynamics of the reduced 3D strong-field process by calculating the electron - ion-core entanglement of the strong-field process in direction $z$. The relevant equations of an interacting electron - ion-core system were introduced in Section 1.3, where the wave function $\Psi^{1 \mathrm{D}}(z, t)$ is actually the wave function of the relative part. Here we work in the same theoretical model as in Chapter 5, particulary as in Section 5.3, where we introduced our earlier one-dimensional results using the 1D Dirac-delta potential.

In Fig. 6.11, we plot the von Neumann entropy $S_{c, z}(t)$ of the density matrix $\varrho_{c, z}\left(z_{c}^{\prime}, z_{c}\right)$ based on Eqs. (5.12) and (5.21), obtained using the indicated improved model potentials for the 1D simulations, and in comparison with the 3D reference simulations. We do this for two different values of the $F$ electric field strength parameter in Fig. 6.11 (a) and (b), which correspond to the tunnel ionization $(F=0.05)$ and to the over-the-barrier ionization regime $(F=0.1)$, respectively.

In Fig. 6.11 (a) we can see that the entropy curves based on our improved model potentials follow the 3D reference results very closely. Here, our density-based model potential is somewhat better, by the virtue of having better representation of the reduced $3 \mathrm{D}$ ground state. The offset of the initial entropy stems from the mixedness of the 3D relative density matrix $\varrho_{z}\left(z^{\prime}, z\right)$, as we have seen it in Section 5.4.3, which is not present in 1D by construction. Despite this offset of values, we can see a striking similarity between these curves, which indicates that the induced entanglement dynamics are quantitatively very close in all of the cases. This is further demonstrated in Fig. 6.11 (b) : we can see that even with stronger ionization, the 1D density- 
(a)

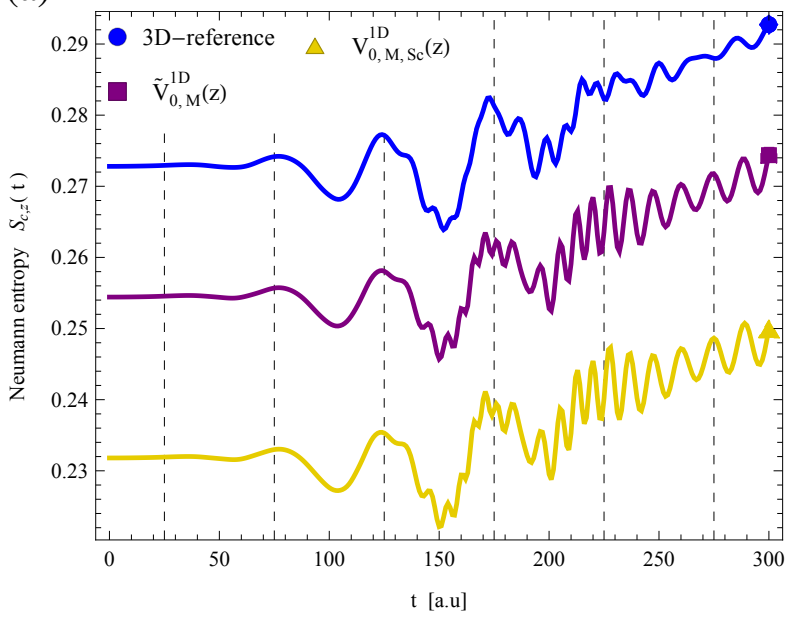

(b)

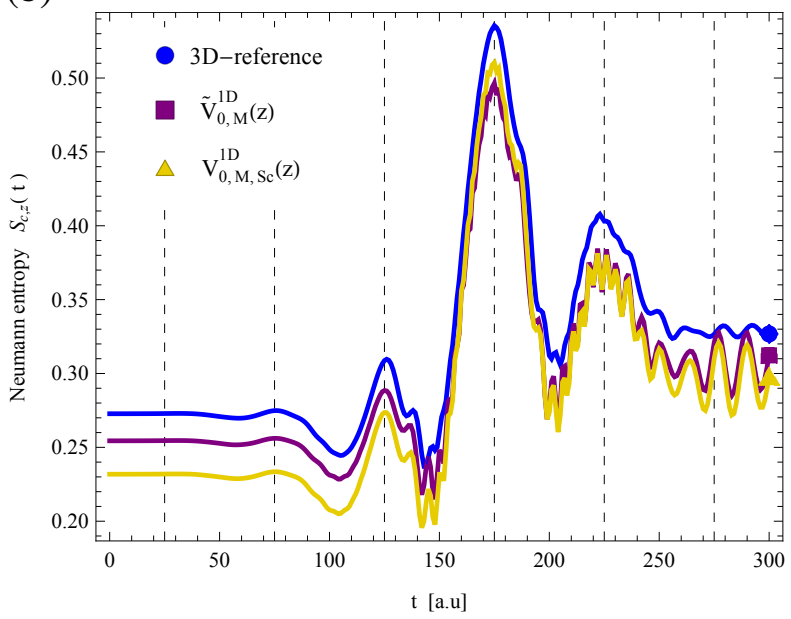

Figure 6.11: Time dependence of the von Neumann entropies $S_{c, z}(t)$ using different 1D model potentials, under the influence of the external field with $F=0.05$ (a) and $F=0.10$ (b), $N_{\text {Cycle }}=$ 3 and $T=100$. Note that each Neumann entropy $S_{c, z}(t)$ quantifies the electron - ion-core entanglement in the direction $z$ reasonably well.

based potentials still give quantitatively correct results without an increase in deviations, where the results of the density-based model potential is still slighly better. In $1 \mathrm{D}$, we can also link this entanglement entropy to the mean values $\left\langle v_{z}\right\rangle(t)$ of Fig. 6.5 (b), just like in the 3D case.

These results about deeply quantum mechanical features demonstrate that our novel model potentials give reliable and physically correct results on the wave function level. The improved soft-core Coulomb potential (6.10) still remains the optimal choice for 1D numerical simulations.

\subsection{Summary}

The results presented in this chapter demonstrate that it is possible to quantitatively model the true $3 \mathrm{D}$ quantum dynamics with the help of the density-based $1 \mathrm{D}$ model potential $\widetilde{V}_{0, \mathrm{M}}^{1 \mathrm{D}}\left(z_{i}\right)$ and the accordingly improved soft-core Coulomb potential $V_{0, \mathrm{M}, \mathrm{Sc}}^{1 \mathrm{D}}(z)$. The best results for the time-dependent physical quantities are obtained with the improved soft-core Coulomb potential (6.10) which is also very easy to use numerically. This means that we can perform quantum simulations of a single-active-electron atom driven by a strong linearly polarized laser pulse during a couple of minutes and obtain a fairly accurate low-frequency response in direction $z$ and a reliable HHG spectrum with the help of the scaling function (6.18). The simple form of this scaling is based on the good agreement between the structure and phase of the 1D and the 3D HHG spectra.

In achieving these results, the physical requirement about the $1 \mathrm{D}$ and $3 \mathrm{D}$ ground state densities was the important starting idea. This led to the construction of the density-based 1D model potential, which then inspired the improved parametrization of the 1D soft-core Coulomb potential with effective charge $\frac{1}{2} Z$. Both of these have the same asymptotic tail which ensures that their ground state energy is identical to that of the 3D system.

The discretization of the density-based 1D model potential gave important lessons also about 
the numerical aspects of nondifferentiable 1D Coulomb-like potentials and the 1D Dirac-delta potential: our numerical construction methods yields $\Delta z^{4}$ convergence for the former type of model potentials, and $\Delta z^{2}$ convergence for the delta potential. It is an important feature that the Hamiltonian matrix of the latter is nonsingular.

Considering the obvious differences between the 1D and the 3D quantum dynamics and their effects, discussed already in connection with Figs. 6.5 (b) and 6.8 (a), it is not surprising that the high-frequency response of these 1D simulations is much stronger than that of the corresponding 3D case. The fact that the scaling function (6.18) has different frequency dependence in the lower frequency domain than in the higher frequency domain, and that this seems to be independent of the other physical parameters, may hint at a deeper connection between the true 3D quantum dynamics and its best 1D model given by the improved soft-core Coulomb potential (6.10). 


\section{Summary of the thesis}

\section{Introduction}

Pioneering experiments with attosecond light pulses [1-6], based on high-order harmonic generation (HHG) in noble gases [7, 9], have been revolutionizing our view of fundamental atomic, molecular and solid state processes in this time domain [11]. A key step in gas-HHG is the tunnel ionization of a single atom and the return of the just liberated electron to its parent ion due to the strong, linearly polarized femtosecond laser pulse driving this process $[17,53]$. Recent developments in attosecond physics revealed that the accurate description of this single-atom emission is more important than ever, especially for the correct interpretation of the experimental data obtained by attosecond metrology.

Although an intuitive and very successful approximate analytical solution [19] and many of its refinements exist [21], the most accurate description of the single-atom response is given by the numerical solution of the time-dependent Schrödinger equation (TDSE). The peculiarity of this problem is due to the electric field strength of the laser pulse, which has its maximum typically in the range of 0.05-0.1 atomic units, i.e. it enables the tunneling of the electron through the time-dependent potential barrier formed by strongly distorting the atomic potential, but this effect is weak during the whole process. On the other hand, this small part of the wave function outside the barrier extends to large distances and in fact this is the main contribution to the time-dependent dipole moment, which is the source of the emitted radiation. Thus, a very weak effect needs to be computed very accurately, and these requirements get even more severe, if the model goes beyond the usually employed single-active-electron and dipole approximations.

For linearly polarized pulses, the main dynamics happens along the electric field of the laser pulse which underlies the success of some one-dimensional (1D) approximations. These typically use various 1D model potentials to account for the behavior of the atomic system. However, the particular model potential chosen heavily influences the 1D results and their comparison with the true three-dimensional (3D) results is usually nontrivial. Therefore, a more elaborate connection between the 3D problem and its $1 \mathrm{D}$ model is necessary to allow the $1 \mathrm{D}$ simulation of strong-field processes physically as correctly as possible.

Although strong-field ionization is widely used as a standard procedure for high-order harmonic generation, it is very little known that this process generates also quantum entanglement between the liberated electron and its parent ion-core. Quantum entanglement is a fundamental feature of quantum theory which enables strong correlations without classical counterpart between constituents of a quantum system. Despite the fact that its discussion dates back to the 
early days of quantum theory [36], the features of continuous variable quantum entanglement [38] are still much less explored and utilized than those of discrete variables systems. Entanglement between the fragments of an atomic system due to a light-induced break-up process, like photoionization and photodissociation, was studied by Fedorov and coworkers $[50,51]$ in the framework of Gaussian states. However, this latter approach is not suitable to deal with the problem of quantum entanglement during the strong-field ionization of an atom, which motivated us to perform an accurate numerical investigation of the problem.

\section{Objectives}

We adopted the usual semiclassical description of the light-matter interaction for the modeling of strong-field phenomena described in the Introduction, making also use of the dipole approximation and choosing the length gauge [57]. In dipole approximation, the spatial variations of the electromagnetic field are neglected in the spatial domain of the atomic processes which is true in the frequency range relevant for the phenomena treated in this thesis. We aim to investigate the effects of a linearly polarized few- or single-cycle femtosecond laser pulse, which has a near-infrared carrier frequency. Our motivation is that this type of laser pulse is short enough to give a more complex response than a simple plane wave. The form of the Hamiltonian suggests the solution of the 3D time-dependent Schrödinger equation in cylindrical coordinates $z$ and $\rho$, where the $z$-axis coincides with the polarization of the laser pulse. The resulting time evolution preserves the magnetic quantum number $m$ and the initial axial symmetry of the wave function if given.

Our first objective was to construct a novel numerical method that is capable to compute the time propagation of the electron's wave function by solving this cylindrical TDSE with highorder accuracy, incorporating also the singularity of the atomic Coulomb potential. Our aim was to construct an algorithm that scales approximately linearly with the total number of spatial gridpoints and that supports parallelization, whenever its possible.

In our earlier work [A1], we modeled a single-active-electron atom as a two-body system in one spatial dimension, using the Dirac-delta potential as the atomic model potential. We verified a strong correlation between the shape of the laser pulse and the oscillations of the quantum entanglement, most importantly, the local maxima of the quantum entanglement coincide with the zero crossings of the laser electric field. A straightforward question is, whether such correlations are also present in the strong-field ionization of a real atom? In accordance with this, our second objective was the analysis of the quantum entanglement properties of a single-electron atom in 3D under strong-field ionization as also mentioned in the Introduction. In this description we modeled a single-electron atom as an interacting two-body system of the electron $(e)$ and the ion-core $(c)$ subsystems. Even though the numerical solution can be carried out, the computational cost of calculating the true electron - ion-core entanglement is prohibitive. We aimed to give an approximate solution for this problem by a directionally separable approximation, and this way to gain insight also into the intricacies and the structure of the pair correlations between the electron's and ion-core's coordinates $z_{e}-z_{c}$ and $x_{e}-x_{c}$.

Our third objective was to find a one-dimensional quantum mechanical model that is capable of providing quantitatively good agreement with the true 3D solution of strong-field processes, 
reduced to the $z$ axis. The one-dimensional description should use only the $z$ Cartesian coordinate, with the very same laser electric field term in the time-dependent Schrödinger equation as in $3 \mathrm{D}$, but the atomic potential should be replaced with a suitable time-independent $V_{0, \mathrm{M}}^{1 \mathrm{D}}(z)$ atomic model potential. We aimed to derive an analytic form for this model potential based on a clear physical principle, and to compare it to well-known existing model potentials like e.g. the $1 \mathrm{D}$ soft-core Coulomb model potential $V_{\mathrm{Sc}}^{1 \mathrm{D}}(z)=-1 / \sqrt{z^{2}+2}$. We also aimed to test the improved model potential rigorously, focusing especially on the particular system's strong-field ionization features.

\section{Methods}

For the numerical solution of the time-dependent Schrödinger equation, we use a combination of various methods. For the spatial derivatives, we use the high-order finite difference formulation [68] with equal grid spacing $\Delta z, \Delta \rho$ in cylindrical coordinates. For the time evolution algorithm, we perform steps in small time intervals $\Delta t$ using the short-time approximation of the time evolution operator $e^{-i \Delta t H_{k}}$ with the second-order effective Hamiltonian $H_{k}^{(2)}$ at the $k$ th time step. We also utilize the second-order Padé-approximation of the exponential operator $e^{-i \Delta t H_{k}^{(2)}}$, which is the usual Crank-Nicolson method [67]. The latter is an implicit method, which means that it involves the solution of a systems of linear equations at every time step, the coefficient matrix of which includes the spatially discretized Hamiltonian matrix. The great advantage of this method is that it also allows us to incorporate any boundary condition into the implicit equations, thus allowing high-order spatial accuracy for a wide variety of problems. For gaining the speed that we need, we also utilize the split-operator methods [89]: by splitting the effective Hamiltonian as $H_{k}^{(2)}=H_{A}+H_{B}$, these methods factorize the above exponential operator into parts that are easy to solve. The most famous of this formula is the second-order accurate symmetric splitting $U_{2}(t+\Delta t, t) \approx e^{-i \frac{\Delta t}{2} H_{B}} e^{-i \Delta t H_{A}} e^{-i \frac{\Delta t}{2} H_{B}}$. The main advantage is that we can achieve linear scaling with the number of gridpoints (if we split kinetic energy terms in the Hamiltonian directionwise). The disadvantage is that it is not possible to split the Hamiltonian operator this way, if it is singular or "very sharp" at a gridpoint. The final relevant splitting method is of Bandrauk and Shen [87], they give a formulation how it is possible to achieve high-order $\Delta t$ convergence by performing a series of back-and-forth substeps with any properly constructed second-order accurate short-time evolution operator.

Deriving the formulas of the numerical method is hardly enough to determine its actual accuracy, rigorous testing is necessary in most cases. Our method of testing the spatial discretization involves computing the eigenenergy of the ground state and at least one excited state of known problems and comparing these values to other accurate numerical or analytical solutions. By changing the discretization parameter $\Delta z$ (or $\Delta \rho)$ we can determine the convergence order of the discretization scheme. These are straightforward typically for systems that have an analytic solution, like the harmonic oscillator and (most importantly) the Coulomb problem. However, analytical solution is not available for most of the time-dependent problems, thus we compare the results to a converged numerical solution that has orders of smaller numerical errors than the setup that we are investigating. The most straightforward method is to compare 
time-dependent mean values of these two solutions, which is an efficient way of error determination dependent on $\Delta t$, but it can be also used to test the spatial accuracy with a given $\Delta z$ (and $\Delta \rho)$ in these situations.

The problem of the interacting two-body quantum system, consisting of the electron $(e)$ and the ion-core $(c)$ as subsystems, is conventionally solved by transforming the system into the center-of-mass reference frame, where the wave function becomes separable in center-of-mass and relative coordinates. We describe the center-of-mass part as a free localized Gaussian wave packet, and the relative part involves the strong-field simulations of the relative particle with coordinates $z, \rho$. The form of governing Schrödinger equation is not changed, only the particle mass is replaced by the reduced mass. During the calculations of physical quantities we also also utilize axial symmetry around $z$ axis, which means that dynamics is the same in the $x$ and $y$ directions for each particle.

For the quantification of the quantum entanglement between the electron and ion-core motion, the usual way requires the density matrix of either the electron or the ion-core by performing partial trace of the other degrees of freedom, assuming the whole quantum system is in pure state. Then the lack of purity of the density matrices is an indicator of quantum entanglement, and can be quantified by calculating the Neumann entropy - amongst other types of quantum entropies - of either subsystem's density matrix. If we can perform this at every time instant we can see the time-dependent entropy dynamics of two particles' entanglement, which is unfortunately a feasible computation in one dimension only. In three dimensions, this type of bipartite approach can be applied to the cylindrical relative coordinates $z, \rho$, by performing the partial trace on one of these coordinates, which gives interesting information about the directional nonseparability of the strong-field process. However, the composite system of these two particles has six degrees of freedom in $3 \mathrm{D}$, which implies a more complex correlation structure between their individual coordinates $z_{e}-z_{c}$ and $x_{e}-x_{c}$. We investigated the nature of these correlations using recent results of quantum information theory [104]. One can quantify these directional pair correlations generally using the so-called $S(e: c, t)$ quantum mutual entropy, and investigate the behavior using the $S(e \mid c, t)$ or $S(c \mid e, t)$ quantum conditional entropies, which latter characterize the remaining entropy of one subsystem if the other one is measured. In the classical limit these two satisfy the relations in classical information theory, however quantum entanglement introduces nonclassical values to them.

For the low-dimensional modeling of an atom, we used the elements of the density functional theory [118]. We derived our model potential in analogy with the the exact calculation of the Kohn-Sham potential of a helium atom with a single Kohn-Sham orbital: knowing the correct reduced (single-particle) density one can invert the Schrödinger equation to determine the Kohn-Sham potential which ensures that the resulting Hamiltonian's ground state has the correct reduced density. In this way one can model the ground state of the system physically as accurately as it is possible with a single orbital.

\section{Scientific results}

In the following, I present a brief summary of my new scientific results discussed in the thesis which are collected in five thesis points. The publications connected to my statements are listed 
at the end of this thesis and cited in each title.

\section{T1. The hybrid splitting algorithm for the solution of the three-dimensional time-dependent Schrödinger equation with Coulomb singularities [P1]}

To treat the Coulomb singularities in the 3D Schrödinger equation using cylindrical coordinates, I derived the formula of the boundary condition of the Coulomb potential in the axially symmetric case, which is a Robin type boundary condition at the $\rho=0$ axis. I also gave the discretized formulation of this boundary condition using one-sided finite difference formulas.

Based on the 4th-order finite difference discretization of the Hamiltonian in the CrankNicolson method, I created the method of hybrid splitting. This uses partial directional splitting of the short-time evolution operator based on the splitting of $H_{k}^{(2)}=H_{A}+H_{B}$ both according to spatial directions and spatial domains: the $H_{A}$ near $\rho=0$ is left intact to retain spatial numerical accuracy near the boundary, while in the outer domain $(\rho \gtrsim 1)$ the $z$-component of the kinetic energy is moved into $H_{B}$.

I created an optimized algorithm to solve the special block pentadiagonal system of linear equations that is provided by the Crank-Nicolson approximation of the central exponential operator $e^{-i \Delta t H_{A}}$. This algorithm reduces the computation costs by $N_{\rho}^{2}$ where $N_{\rho}$ is the number of the discretization points along the $\rho$ direction.

I verified that the discretization scheme is 4th-order accurate in spatial steps $\Delta z$ and $\Delta \rho$ by computing the eigenvalues of the Coulomb and the harmonic oscillator Hamiltonians. Using the time-dependent analytic solution of the forced harmonic oscillator, I verified that the hybrid splitting method is $\Delta t^{2}$ accurate, and that it can be successfully combined with the 4th-order approximation of the evolution operator to become $\Delta t^{4}$ accurate in temporal steps.

\section{T2. Quantification of the electron - ion-core quantum entanglement during strong-field ionization [P2]}

I showed that the computation of the Neumann entropy in a 3D simulation of strong-field ionization of the electron - ion-core system is not feasible due to the prohibitively large numerical load of the problem. Therefore, I created the following procedure to characterize the quantum entanglement of the electron - ion-core system in a 3D strong-field simulation.

First I reduced the 3D dynamics along spatial directions (parallel and perpendicular to the polarization of the laser pulse) by partial tracing of the respective density matrices. Then I performed the transformation in each direction to electron and ion-core coordinates and reduced these density matrices to single-particle density matrices by tracing over the respective particle coordinates. Using known relations of quantum entropies, I showed that the type of the correlation between the same coordinates of these two particles in each direction is dominantly quantum entanglement. I quantified this directionwise quantum entanglement via the average directional mutual entropy. I verified that the time evolution of the average mutual entropy along the polarization direction is very similar to that of the exact quantum entanglement entropy obtained by my former 1D model simulations. 
Using these density matrices as building blocks, I approximated the quantum state of the 3D electron - ion-core system by the product of the spatially reduced two-particle density matrices, in accordance with my experience that the strong-field dynamics along the above spatial directions is weakly coupled.

Based on this, I defined the approximate entanglement entropy of the 3D electron - ion-core system by adding up the directional average mutual entropies individually, which thus can be efficiently computed based on the entropies of the building block density matrices. I showed via simulations that this approximated entanglement entropy satisfies the strong subadditivity relation, which is an analytic property of the exact entanglement entropy.

\section{T3. Features of the electron - ion-core quantum entanglement during strong-field ionization [P2]}

Since the spatially reduced subsystems described in $\mathbf{T 2}$ are in mixed states, their correlations are more complicated than pure state entanglement, thus I identified the physical meaning of the several kinds of entropies involved in the above procedure and I made the following observations.

I analyzed the entanglement entropy relations in each of the directions specified in $\mathbf{T} 2$ and I found that their local maxima almost coincide with the zero crossings of the electric field of the laser pulse. The entanglement along the directions parallel and perpendicular to the laser polarization are very similar to each other if the process stays in the tunnel ionization regime. However, in the over-the-barrier ionization regime, I found entropy increase along the parallel direction but a surprising entropy decrease in the perpendicular directions which causes also the total electron - ion-core entanglement entropy to decrease.

I investigated the dependence of these proposed measures of entanglement dynamics on the strength and the carrier-envelope phase of the driving laser pulse. I found many features of quantum entropies that do not depend on these parameters, like the electron - ion-core entanglement has local maxima always near the zero crossings of the laser pulse. I found that while the intensity of the field governs the dynamics as a whole, the carrier-envelope phase changes the subcycle dynamics of the strong-field ionization.

\section{T4. One-dimensional density-based model potentials: comparison of the 1D and 3D results [P3]}

I derived the analytic formula of a 1D atomic model potential that by definition has the same ground state probability density as the probability density of the 3D Coulomb problem integrated over $\rho$. I determined that through asymptotics this $1 \mathrm{D}$ system preserves the 3D ground state energy if the source 3D problem has a long-range Coulomb form. The densitybased potential consists of a 1D regularized Coulomb potential plus a kinetic energy correction.

I realized that the regularized Coulomb potential part of the density-based model potential suggests the value of $\frac{1}{2} Z$ for the effective ion-core charge in 1D. Based on this, I proposed improved formulas of the $1 \mathrm{D}$ soft-core Coulomb potential and the 1D regularized Coulomb 
potential by applying the effective charge $\frac{1}{2} Z$ while maintaining that their ground state energies equal to that of the 3D Coulomb problem.

I showed by direct comparison of simulation results of typical time-dependent strong-field processes, driven by a linearly polarized near-infrared laser pulse, that both these new and the proposed improved 1D model potentials exhibit an impressive enhancement in the accuracy of the low frequency response of typical strong-field processes by capturing the essence of the real 3D dynamics. These tests also showed that the best model potential quantitatively is the improved soft-core Coulomb potential.

I also computed the dipole power spectrum in a wide variety of cases and I observed that the structure of the spectra based on the density-based and improved 1D model potentials is remarkably similar to those based on the 3D simulations. The match of the corresponding spectral phases is also very good, especially in the higher frequency range, which is of fundamental importance for the generation of isolated attosecond pulses. I gave a simple frequency-dependent scaling function that proved to be capable to convert the 1D spectra to the corresponding 3D spectra in all of the tested cases, thus it enables to compute the dipole power spectra via 1D simulations.

\section{T5. Improved numerical method of constructing discrete model potentials in one dimension: improved simulation accuracy [P3]}

I suggested a formula to acquire an improved discretized representation of 1D model potentials, based on the potential's ground state and ground state energy, by inverting the discretized time-independent Schrödinger equation. The resulting discretized potentials have the numerically exact ground state and ground state energy. I showed that if the exact 1D potential is not differentiable at some spatial point (e.g. like a regularized 1D Coulomb potential) then the resulting discretized Hamiltonian is still $\Delta z^{4}$ accurate, if the finite difference formulas of the partial derivatives is also at least $\Delta z^{4}$ accurate, even during simulations of strong-field ionization phenomena.

I showed that the application of this method is possible also for the 1D Dirac-delta potential. The same inversion formula as above gives a nonsingular discretized model potential. I tested the results using convergence tests of strong-field simulations, the numerical errors at $\Delta z=$ 0.2 were comparable to the correct method used in [A1]. I came to the conclusion that this nonsingular method of discretization converges to the true solution, and it shows $\Delta z^{2}$ numerical accuracy. 


\section{Összefoglalás}

\section{Bevezetés}

Az elmúlt másfél évtizedben az attoszekundumos fényimpulzusokkal [1-6] elvégzett úttörő kísérletek forradalmasították az ezen az idôskálán lezajló alapvetô atomi, molekuláris és szilárdtestfizikai folyamatokkal kapcsolatos tudásunkat [11]. Ezen attoszekundumos fényimpulzusok előállításának alapja a nemesgázokban fellépô magasrendú felharmonikus keltés (HHG) [7, 9], aminek kulcslépései az atom alagutazásos ionizációja majd az így kiszabadult elektron visszatérése az iontörzshöz, amelyeket egy erős, lineárisan polarizált femtoszekundumos lézerimpulzus elektromos mezője vezérel $[17,53]$. A legújabb fejlemények az attoszekundumos fizikában rámutatnak, hogy az egy atom általi emisszió pontos leírása fontosabb mint valaha, különösen az attoszekundumos méréstechnika által nyújtott kísérleti adatok helyes értelmezéséhez.

Habár létezik egy intuitív és nagyon sikeres közelítő analitikus megoldás [19] illetve ennek továbbfejlesztései [21], az egyatom válasz legpontosabb leírását a megfelelő időfüggő Schrödinger-egyenlet (TDSE) numerikus megoldása adja meg. A probléma egyik sajátossága a lézerimpulzus elektromos terének erôssége, amelynek maximuma tipikusan 0.05-0.1 atomi egység tartományban van: ez lehetôvé teszi az elektron alagutazását az erôsen torzított atomi potenciál által alkotott időfüggő potenciálgáton keresztül, azonban ez a hatás gyenge az egész folyamat alatt. Másrészről viszont, a hullámfüggvény ezen kis része a potenciálgáton kívül nagy távolságokra jut el, ezért ez adja a fố hozzájárulást a kibocsátott sugárzás forrásához, az időfüggő dipólus momentumhoz. Így egy nagyon gyenge hatást szükséges nagyon pontosan kiszámítani, és ezeket a követelményeket még fokozottabban kell figyelembe venni, ha a modell túlmegy a szokásosan használt egy aktív elektron és dipól közelítéseken.

Lineárisan polarizált impulzusok esetén a fố dinamika az elektromos tér polarizációjának irányában történik, ez alapozza meg néhány egydimenziós (1D) közelítés sikerességét. Ezek a modellek tipikusan különféle 1D atomi modell potenciálok használatával próbálnak számot adni az atomi rendszer viselkedéséről. Azonban a választott atomi modell potenciál nagyban befolyásolja az 1D eredményeket, és azok közvetlen összehasonlítása az igazi háromdimenziósakkal (3D) általában nem triviális. Ezért egy jobban megalapozott kapcsolat szükséges a 3D probléma és annak 1D modellje között annak érdekében, hogy az erôs teres folyamatok 1D szimulációit fizikailag a leghelyesebben végezhessük el.

Habár az erôs teres ionizáció egy széleskörúen használt standard eljárás a magasrendú felharmonikusok előállításához, az kevéssé ismert, hogy ez a folyamat a kiszabadított elektron és az ion-törzs között kvantumos összefonódottságot hoz létre. A kvantumos összefonódottság egy 
alapvető jellegzetessége a kvantumelméletnek, amely erôs korrelációt képez a kvantumrendszer alkotói között és nincs klasszikus megfelelője. Dacára annak, hogy ennek fontosságát már a kvantum elmélet korai idôszakában is felismerték [36], a folytonos változójú kvantumrendszerek összefonódottságnak tulajdonságai [38] jóval kevésbé felderítettek és felhasználtak, mint a diszkrét változójú rendszerek esetében. Fény hatására - pl. fotoionizáció vagy fotodisszociáció által - szétesố atomi rendszer fragmentumai közötti összefonódottság is ilyen, amelyet Fedorov és munkatársai $[50,51]$ vizsgálták Gauss állapotok segítségével. Azonban ez a megközelítés nem megfelelő az elektron-iontörzs összefonódottság vizsgálatára erôs teres ionizáció során, ami erős motivációt adott számunkra, hogy elvégezzük a probléma pontos numerikus vizsgálatát.

\section{Célkitúzések}

A már a Bevezetésben említett erôs teres jelenségek tárgyalása során a fény által okozott hatásokat a szokásos szemiklasszikus módon írjuk le, amelyben felhasználjuk a dipól közelítést és ún. hossz-mértéket választunk [57]. Dipólközelítésben az elektromágneses mező térbeli változásait elhanyagoljuk az atomi folyamatok környezetében, amely igaz abban a frekvencia tartományban amely lényeges ezen doktori disszertációban tárgyalt folyamatok szempontjából. Célunk, hogy egy lineárisan polarizált, közeli infravörös vivőfrekvenciával rendelkezô, egyvagy néhány ciklusú lézerimpulzus által indukált erôs teres ionizációt vizsgáljunk. Egy ilyen lézerimpulzus rövid, de sokkal összetettebb választ generál mint egy egyszerú síkhullám. A megfelelő Hamilton operátor alakja alapján a 3D időfüggő Schrödinger-egyenletet a $z$ és $\rho$ hengerkoordinátákban célszerú felírni, ahol a z-tengely egybeesik a lézerimpulzus polarizációjának irányával. Az időfejlődés megôrzi az $m$ mágneses kvantumszámot és a hullámfüggvény adott kezdeti forgásszimmetriáját.

Az első célkitűzésünk, hogy egy olyan új numerikus módszert alkossunk, amely képes az elektron hullámfüggvényének időfejlesztésére a hengerkoordinátákban felírt időfüggő Schrödinger-egyenlet nagy pontosságú megoldásával, figyelembe véve az atomi Coulomb potenciál szingularitásának hatását. Célunk továbbá, hogy a vázolt algoritmus számítási igénye lineárisan skálázódjon a térbeli rácspontok számával, és legyen párhuzamos szálakra bontható, amikor csak lehetséges.

Korábbi munkánkban [A1] egy egyelektronos atomot modelleztünk kéttest rendszerként egy dimenzióban a Dirac-delta potenciált mint atomi modell potenciált felhasználva. Erôs korrelációt találtunk a lézerimpulzus idôbeli alakja és az ebben a rendszerben lévő kvantumos összefonódottság oszcillációi között, az összefonódottság lokális maximumai egybeestek a lézerimpulzus elektromos terének zéróhelyeivel. Logikus kérdés, hogy ilyen korrelációk jelen vannak-e egy igazi atom erős teres ionizációja esetében is? Ennek megfelelően a második célkitúzésünk, hogy elvégezzük az egyelektronos atomok kvantumos összefonódottsági tulajdonságainak részletes vizsgálatát a Bevezetésben említett erős teres körülmények között. Ebben a leírásban az egyelektronos atomot elektron ( $e$ ) és iontörzs ( $c$ ) alrendszerekból álló kéttest problémaként modellezzük. Habár ezen probléma esetén a numerikus időfejlesztés elvégezhetô, az elektron iontörzs összefonódottság mértékének a számítási igénye olyan nagy, ami lehetetlenné teszi a kiszámítását értelmes idô alatt. Ezért azt tûztük ki célunkként, hogy adjunk egy közelítô megol- 
dást erre a problémára, az irányok mentén szeparált közelítés által, és így betekintést nyerjünk a $z_{e}-z_{c}$ és $x_{e}-x_{c}$ elektron és iontörzs koordináták közötti párkorrelációk felépítésébe.

Harmadik célkitűzésünk, hogy találjunk egy olyan egydimenziós atomi modell potenciált, ami képes kvantitatívan is jó egyezést nyújtani az igazi 3D erős teres folyamat $z$-tengelyre redukált megoldásával. Az egydimenziós leírás csak a $z$ Descartes koordinátát tartalmazza, ugyanazzal a lézer elektromos tere által adott taggal az időfüggő Schrödinger-egyenletben mint 3D esetén, viszont a megfelelően választott új, időfüggetlen $V_{0, \mathrm{M}}^{1 \mathrm{D}}(z)$ atomi modell potenciállal. Célunk volt levezetni egy analitikus potenciál formát tiszta fizikai elvekre alapozva, majd összehasonlítani ezt a már jól ismert modell potenciálokkal, pl.: az egydimenziós $V_{\mathrm{Sc}}^{1 \mathrm{D}}(z)=-1 / \sqrt{z^{2}+2}$ soft-core Coulomb modell potenciállal. Célunk, hogy az így kapott 1D-s modell potenciálok eredményeit szigorúan és részletekbe menően teszteljük, különösen az adott rendszer erős teres ionizációjának tulajdonságaira fókuszálva.

\section{Módszerek}

Az időfüggő Schrödinger-egyenlet numerikus megoldásához több módszer kombinációját használtuk fel. A tér szerinti deriváltak diszkretizálására magasrendú véges differencia formulákat [68] használunk $\Delta z, \Delta \rho$ egyenközú rácsbeosztásokkal hengerkoordinátákban. Az időfejlesztő algoritmust kis $\Delta t$ lépésekben hajtjuk végre, felhasználva az evolúciós operátor $e^{-i \Delta t H_{k}}$ kis időintervallumra érvényes alakját, itt $H_{k}^{(2)}$ a $k$-dik lépés másodrendû effektív Hamilton operátora. Felhasználjuk továbbá a $e^{-i \Delta t H_{k}^{(2)}}$ exponenciális operátor másodrendú Padé-közelítését, amely a szokásos Crank-Nicolson módszert adja [67]. Ez utóbbi egy implicit módszer, amely azt jelenti, hogy minden időléptetés egy lineáris egyenletrendszer megoldásával jár, és ezen egyenletrendszer együtthatómátrixa tartalmazza a térben diszkretizált Hamilton mátrixot. Ennek a módszernek nagy előnye, hogy lehetôvé teszi bármilyen peremfeltétel beépítését az implicit egyenletekbe, amely magasrendú térbeli pontosságot tesz lehetôvé problémák széles köre esetében. A futtatások sebességének javítása érdekében felhasználjuk a split-operátor módszert [89]: azzal, hogy felbontjuk az effektív Hamilton operátort mint $H_{k}^{(2)}=H_{A}+H_{B}$, ez a módszer olyan szorzattá alakítja a fenti exponenciális operátort, amelynek tényezőit könnyú hattatni. A legismertebb ilyen formula a másodrendú szimmetrikus felbontás $U_{2}(t+\Delta t, t) \approx e^{-i \frac{\Delta t}{2} H_{B}} e^{-i \Delta t H_{A}} e^{-i \frac{\Delta t}{2} H_{B}}$. A fő előnye, hogy ezzel a módszerrel elérhetjük azt, hogy a múveleti igény a térbeli rácspontok számával lineárisan skálázódjon (ha irányonként szétválasztjuk a Hamilton operátorban lévő kinetikus energia tagokat). A módszer hátránya az, hogy nem felbontható ilyen úton a Hamilton operátor, ha az szinguláris vagy „,nagyon éles” egy adott rácspontban. Az igazán releváns splitoperátor módszer Bandrauk és Shen [87] eredménye: az általuk adott formulák lehetôvé teszik magasrendú $\Delta t$ konvergencia elérését azáltal, hogy a megadott módon előre-hátra idôléptetünk a megfelelően konstruált, másodrendben pontos kis időlépéses evolúciós operátorral.

A numerikus módszer formuláinak levezetése azonban általában nem elégséges ahhoz, hogy meghatározzuk a módszer valódi pontosságát, ezért szigorú tesztelés szükséges a legtöbb esetben. A tér szerinti deriváltak pontosságát tesztelő egyik módszerünkben kiszámoljuk ismert problémák alapállapoti és legalább egy gerjesztett állapoti sajátenergiáját numerikusan, és összehasonlítjuk azokat ismert analitikus vagy más nagyon pontos numerikus megoldásokkal. A $\Delta z$ 
(vagy $\Delta \rho$ ) diszkretizációs paraméter változtatásával meg tudjuk határozni a térbeli diszkretizációs módszer konvergenciájának rendjét. Ez a megközelítés egyszerú olyan rendszerekre, amelyek rendelkeznek analitikus megoldással, például a kvantum harmonikus oszcillátor és a Coulomb probléma. Azonban analitikus megoldás nem ismert a legtöbb időfüggô probléma esetében, ekkor az eredményeket egy már konvergáltnak tekinthető numerikus megoldással vetjük össze, amely utóbbinak numerikus hibái nagyságrendekkel kisebbek mint a beállítás amelyet vizsgálunk. Ennek legegyszerúbb útja az, hogy összehasonlítjuk a két megoldás időfüggő várható értékeit, amely a $\Delta t$ függő hibák hatékony meghatározási módját jelenti, de a térbeli pontosságot is tesztelhetjük vele a $\Delta z$ (vagy $\Delta \rho$ ) paraméter változtatásával.

Az elektron (e) és az iontörzs (c) alrendszerekből álló kölcsönható kéttest kvantumrendszer problémáját hagyományosan a tömegközépponti vonatkoztatási rendszerben oldjuk meg, ahol a hullámfüggvény szeparálható tömegközépponti és relatív koordináták szerint. A tömegközépponti részt egy szabad lokalizált Gauss hullámcsomagként írjuk le, és a relatív rész foglalja magában a $z, \rho$ koordinátás relatív részecskének erôs teres szimulációit. Az erôs teres időfüggő Schrödinger-egyenlet alakja változatlan, de az elektron tömegét helyettesítjük a $\mu$ redukált tömeggel. Fizikai mennyiségek számolása során továbbá felhasználjuk az állapot kezdeti forgásszimmetriáját a $z$-tengely körül, ami azt jelenti, hogy $x$ és $y$ irányokban a dinamika azonos lesz.

A szokásos módszer az iontörzs és elektron közötti kvantumos összefonódottság mennyiségi jellemzésére az elektron vagy az iontörzs koordinátás egyrészecske súrúségmátrix kiszámítását foglalja magában, amelyet a többi szabadsági fokra vett parciális átlósösszeg (vagyis ezen koordináták szerinti integrálás) után kapunk meg, feltéve, hogy a teljes kvantumrendszer tiszta állapotban van. Ekkor ezen részrendszerek sưrűségmátrixainak kevertsége egyértelmú jellemzője az összefonódottságnak, amelyet kvantiatíve a Neumann entrópiával - általánosabban valamilyen kvantum entrópiával - írunk le, ezt az egyik alrendszer sûrúségmátrixából számítjuk ki. Ha minden egyes idôpontban végre tudjuk ezt hajtani, megkapjuk a két részecske összefonódottságának időfüggő dinamikáját, ez azonban sajnos csak egydimenziós modell esetén keresztülvihetô számítás. Három dimenzióban ez a típusú kétrészú megközelítés gyakorlatilag csak a $z, \rho$ koordinátákra kiszámítható, amely érdekes információkat adhat az erős teres folyamatok irányonkénti nem-szeparálhatóságáról. A kompozit rendszer azonban hat szabadsági fokkal rendelkezik három dimenzióban, amely egy sokkal bonyolultabb korrelációs struktúrát von maga után a $z_{e}-z_{c}$ és $x_{e}-x_{c}$ koordináták között. Ezen korrelációk természetét a kvantum-információelmélet újabb eredményei segítségével [104] vizsgáltuk meg. Ezen irány menti párkorrelációkat mennyiségileg az úgynevezett $S(e: c, t)$ kvantum kölcsönös entrópiával jellemezhetjük, és ezek viselkedését a $S(e \mid c, t)$ vagy $S(c \mid e, t)$ kvantum feltételes entrópiákkal tárhatjuk fel, utóbbi az egyik részrendszer megmaradt entrópiáját jellemzi, amennyiben a másikon teljes mérést hajtottunk végre. Klasszikus határesetben ez a két entrópia a klasszikus információelmélet összefüggéseit teljesíti, azonban a kvantumos összefonódottság nemklasszikus számértékeket tesz lehetôvé számukra.

Atomok alacsony dimenziós modellezéséhez a súrúségfunkcionál elmélet [118] elemeit használtuk fel. A modell potenciálunkat az egyetlen pályával leírt hélium atom egzakt KohnSham potenciáljának analógiája alapján vezetjük le: ismerve a helyes redukált (egyrészecske) sûrűséget, invertálhatjuk a Schrödinger-egyenletet, ami meghatározza ezt potenciált. Ez az elő- 
állítás biztosítja, hogy az így kapott Hamilton operátor alapállapota a helyes redukált sưrúséggel rendelkezzen. Ily módon fizikailag modellezni tudjuk a rendszer alapállapotát amennyire helyesen csak lehet egyetlen hullámfüggvény vagy pálya segítségével.

\section{Tudományos eredmények}

Az alábbiakban röviden ismertetem a disszertációban bemutatott új tudományos eredményeimet öt tézispontban összefoglalva. A megállapításaimhoz kapcsolódó, a a disszertáció végén található listában összegyújtött publikációkra a tézispontok címében hivatkozom.

\section{T1. Hibrid split-operátor algoritmus a Coulomb szingularitásokat tartal- mazó háromdimenziós idôfüggó Schrödinger-egyenlet megoldásához [P1]}

Abból a célból, hogy kezeljem a Coulomb szingularitásokat a háromdimenziós hengerkoordinátás Schrödinger-egyenletben, levezettem a Coulomb potenciál peremfeltételére vonatkozó formulát forgásszimmetrikus esetben, a $\rho=0$ tengely mentén. Megadtam ennek a peremfeltételnek a diszkretizált formáját féloldali véges differencia formulákat használva.

A Hamilton operátor negyedrendû véges differencia közelítése alapján felírtam a hibrid splitting módszerét. Ez a split-operátor módszer a kis időintervallumú evolúciós operátor részleges irány menti felbontásán alapul. Ez azt jelenti, hogy a $H_{k}^{(2)}=H_{A}+H_{B}$ operátor mind térbeli irányok és térbeli tartományok szerinti felbontást is magában foglal: a $H_{A}$ operátor a $\rho=0$ tengelyhez közel megegyezik $H_{k}^{(2)}$-vel azért, hogy ezen perem környékén a módszer megốrizze a numerikus pontosságot, és mindeközben a külső tartományban $(\rho \gtrsim 1)$ a kinetikus energia $z$-komponense átkerül a $H_{B}$ operátorba.

Létrehoztam egy optimizált algoritmust ami megoldja az $e^{-i \Delta t H_{A}}$ központi exponenciális operátor Crank-Nicolson közelítése eredményeképpen előálló speciális blokk pentadiagonális lineáris egyenletrendszert. Ez az algoritmus a számítási igényt $N_{\rho}^{2}$-el csökkenti, ahol $N_{\rho}$ a $\rho$ tengely mentén lévő a rácspontok száma.

Szimulációkkal igazoltam, hogy a fenti diszkretizációs séma $\Delta z$ és $\Delta \rho$ térlépésekben negyedrendben pontos a hidrogén atom és a kvantum harmonikus oszcillátor sajátenergiáinak kiszámolásával. Felhasználva a kényszerített kvantum harmonikus oszcillátor időfüggő analitikus megoldását igazoltam továbbá, hogy a hibrid splitting módszer $\Delta t^{2}$ pontos, és sikeresen kombinálható az evolúciós operátor negyedrendú közelítéseivel, hogy idôlépésben $\Delta t^{4}$ pontosságot nyújtson.

\section{T2. Az elektron - iontörzs kvantumos összefonódottságának kvantitatív jellemzése erôs teres ionizáció során [P2]}

Megmutattam, hogy az iontörzs - elektron rendszer háromdimenziós erôs teres szimulációiban a megfelelő Neumann entrópia numerikus kiszámítása nem keresztülvihetô a probléma rendkívül magas számítási igénye miatt. Ezért megalkottam a következő eljárást a iontörzs és az elektron kvantumos összefonódottságának jellemzésére erős teres folyamatokban. 
Először redukáltam a 3D-s dinamikát a lézerimpulzus polarizációjára párhuzamos és merőleges térbeli irányokra úgy, hogy parciális átlósösszegét vettem a megfelelő sưrúségmátrixoknak. Ezután elvégeztem minden irányban az áttérést elektron és iontörzs koordinátákra és tovább redukáltam ezeket a sűrüségmátrixokat a megfelelő részecskére vett parciális átlósösszeg végrehajtásával. A kvantumentrópiák ismert összefüggéseit felhasználva megmutattam, hogy a két részecske azonos irányú koordinátái közötti korreláció dominánsan kvantumos összefonódottság. Ennek az irány menti kvantumos összefonódottságnak a mértékét az átlagos irány menti kölcsönös entrópiával jellemeztem. Megmutattam, hogy az átlagos kölcsönös entrópia időfejlődése a lézerimpulzus polarizációs irányában nagyon hasonló a korábbi egydimenziós számolásaim során kapott egzakt kvantumos összefonódottsági entrópiák időfejlődéséhez.

Ezeket a súrúségmátrixokat felhasználva, a 3D-s iontörzs - elektron rendszer kvantumállapotát az irányokra redukált kétrészecske sűrúségmátrixok szorzatával közelítettem, arra a szimulációs tapasztalatomra alapozva, hogy az erős teres dinamika a fenti irányok mentén csak gyengén csatolt.

Ezek alapján definiáltam a 3D-s iontörzs - elektron rendszer közelítő összefonódottsági entrópiáját mint az átlagos irány menti kölcsönös entrópiák összegét, amely hatékonyan számolható a már meghatározott irány menti sûrűségmátrixok segítségével. Megmutattam szimulációk segítségével, hogy ez a közelítő összefonódottsági entrópia kielégíti az erős szubadditivitás tételét, amely az egzakt összefonódottsági entrópia fontos analitikus tulajdonsága.

\section{T3. Az elektron - iontörzs kvantumos összefonódottság tulajdonságai erōs teres ionizáció során [P2]}

Mivel a T2 pontban bevezetett térben redukált alrendszerek kevert állapotban vannak, ezért a korrelációs tulajdonságaik sokkal bonyolultabbak, mint tiszta állapoti összefonódottság esetén, ebből adódóan először azonosítottam a fenti eljárással kapcsolatos különféle entrópiák fizikai jelentését. Ezek alapján a következő megállapításokat tettem.

Részletesen elemeztem a összefonódottsági entrópiák összefüggéseit minden egyes irányban a T2 tézispont szerint, és azt találtam, hogy a lokális maximumaik szinte egybeesnek a lézerimpulzus elektromos terének zéróhelyeivel. A lézerimpulzus polarizációjával párhuzamos és merôleges irányokban az összefonódottság nagyon hasonló egymáshoz, amennyiben a lézertér erôssége az alagutazásos ionizációnak megfelelő tartományban van. Azonban potenciálgát feletti ionizáció esetén a polarizációval párhuzamos irányban az összefonódottsági entrópia növekedést, míg a merőleges irányokban ezen entrópia csökkenést mutat, amelyek összhatása csökkenést eredményez a teljes iontörzs - elektron összefonódási entrópiánál.

Megvizsgáltam a fenti javasolt összefonódási dinamika mértékeinek függését a vezérlő lézerimpulzus erôsségétôl és vivő-burkoló fázisától. A kvantumentrópiák időfejlődésében több olyan tulajdonságot is találtam, amelyek nem függenek ezektôl a paraméterektől, például az iontörzs - elektron összefonódottság mindig maximumot ér el a lézerimpulzus zéróhelyei körül. Azt is megfigyeltem, hogy miközben a külső tér intenzitása az erôs teres ionizáció dinamikájának egészét irányítja, addig a vivő-burkoló fázis csak a ciklusokon belüli dinamikát változtatja meg. 


\section{T4. Egydimenziós sứrúség alapú atomi modell potenciálok: 1D és 3D ered- mények összehasonlítása [P3]}

Levezettem annak az egydimenziós atomi modell potenciálnak az analitikus formuláját, amely definíció szerint ugyanazzal az alapállapoti valószínúségi sưrúséggel rendelkezik, mint a háromdimenziós Coulomb probléma $\rho$ változó szerint integrált valószínúségi sûrúsége. Megállapítottam, hogy az aszimptotikus viselkedése által ez az 1D modell megôrzi a 3D probléma alapállapoti energiáját, amennyiben a 3D problémához tartózó potenciál aszimptotikusan Coulomb alakú. Ez az új sưrúség alapú 1D atomi modell potenciál egy regularizált 1D Coulomb potenciálból és egy kinetikus energia korrekcióból áll.

Felismertem, hogy a súrúség alapú 1D atomi modell potenciál regularizált Coulomb része $\frac{1}{2} Z$ effektív magtöltést tartalmaz egyelektronos atomokra (ahol $Z$ az eredeti magtöltés). Ez alapján tökéletesítettem az $1 \mathrm{D}$ soft-core Coulomb és a regularizált 1D Coulomb potenciálra vonatkozó formulákat úgy, hogy $\frac{1}{2} Z$ effektív magtöltéssel rendelkezzenek és az alapállapoti energiájuk maradjon egyenlő a 3D Coulomb problémáéval.

Lineárisan polarizált közeli infravörös lézerimpulzus által vezérelt időfüggő erős teres szimulációk eredményeinek összehasonlításával megmutattam, hogy az új és a tökéletesített modell potenciálok az erôs teres folyamatok alacsony frekvenciás válaszainak tekintetében sokkal pontosabb eredményeket adnak, mert kvantitatíve helyesen modellezik az igazi 3D dinamika lényegi elemeit. Ezek a tesztek azt is megmutatták, hogy ezen potenciálok közül a tökéletesített soft-core Coulomb potenciál adja a legpontosabb közelítést.

Az esetek széles körében kiszámoltam a dipól teljesítményspektrumot és arra a következtetésre jutottam, hogy az 1D súrúség alapú és a tökéletesített modell potenciálok által nyújtott spektrumok szerkezete nagyon hasonló a 3D szimulációkból kapottakhoz. A megfelelô spektrális fázisok egyezése is nagyon jó, különösen a magas frekvenciás tartományban, amely alapvető fontosságú az izolált attoszekundumos lézerimpulzusok előállításának szempontjából. Megadtam egy egyszerú frekvenciafüggố skálafüggvényt, amely képesnek bizonyult a kapott 1D spektrumokat a megfelelő 3D spektrumokká átalakítani a tesztelt esetekben, ami a valódi dipól teljesítményspektrum 1D szimulációkon alapuló mennyiségileg helyes számítását is lehetôvé teszi.

\section{T5. Javított numerikus módszer diszkrét modell potenciálok elôállítására egy dimenzióban: nagyobb szimulációs pontosság [P3]}

Javasoltam egy formulát, amely 1D modell potenciálok jelentősen pontosabb diszkretizált reprezentációját adja meg, a potenciál egzakt alapállapotán és alapállapoti energiáján alapulva, a megfelelő diszkretizált időfüggetlen Schrödinger-egyenlet inverziójának segítségével. Az így kapott diszkretizált potenciálok numerikusan egzakt alapállapottal és energiával rendelkeznek. Megmutattam, hogy ha az egzakt 1D potenciál nem differenciálható egy térbeli rácspontban (pl. az 1D regularizált Coulomb potenciál), akkor az így elóállított diszkrét Hamilton mátrix $\Delta z^{4}$ pontosságú, amennyiben a benne lévő parciális deriváltak is legalább $\Delta z^{4}$ pontossággal rendelkeznek, ami az erős teres szimulációk során is igaznak bizonyult.

Megmutattam, hogy a fenti módszer alkalmazható az 1D Dirac-delta potenciálra is. A fent 
említett inverziós formula ennek a modell potenciálnak egy nemszinguláris diszkretizált reprezentációját adja. Azt kaptam erős teres szimulációkon alapuló konvergencia teszteket elvégezve, hogy a módszer numerikus hibái $\Delta z=0.2$-nél hasonlóak az [A1] cikkünkben használt korrekt módszeréhez. Arra a következtetésre jutottam, hogy ez a nemszinguláris diszkretizált módszer az igazi megoldáshoz konvergál és $\Delta z^{2}$ numerikus pontosságot mutat. 


\section{Acknowledgements}

First of all, I would like to express my sincere gratitude to my supervisor, Attila Czirják for his continuous support during my studies. He always encouraged and trusted me even when I faced the most challenging tasks. I am also grateful to Mihály Benedict who helped me to improve the thesis through constructive critical remarks. His character, as a professor, has also a great impact on me.

I am thankful to the head of department, László Fehér who also made my continuous employment possible. Thanks are also due to Sándor Varró and Katalin Varjú who gave me a very extensive introduction to the theoretical description of intense light-matter interaction and to attosecond physics. I also thank Ferenc Bogár, Gábor Paragi for giving me introduction to molecular physics and density functional theory. Everyone at the Department of Theoretical Physics also have my gratitude for creating an inspiring atmosphere. Particularly, I enjoyed my daily work with the fellow inhabitants of office 230, Viktor Szaszkó-Bogár and István Magashegyi. I am forever indebted to my mathematics and physics teachers, Csaba Rapcsák and Krisztián Váradi in high school for making me interested in mathematics and physics and to their dedication for natural sciences.

Part of this work has been granted by the Hungarian Scientific Research Fund OTKA under contract No. T81364. The project has been also supported by the European Union, co-financed by the European Social Fund, EFOP-3.6.2-16-2017-00005. This work was supported by the GINOP-2.3.2-15-2016-00036 project. Partial support by the ELI-ALPS project is also acknowledged. The ELI-ALPS project (GOP-1.1.1-12/B-2012-000, GINOP-2.3.6-15-2015-00001) is supported by the European Union and co-financed by the European Regional Development Fund. I was also supported by the UNKP-17-3 New National Excellence Program of the Ministry of Human Capacities of Hungary.

Finally, I would like to express my thanks to my family and friends for their unending support. 


\section{Publications}

\section{Refereed research papers related to the thesis:}

[P1] Szilárd Majorosi and Attila Czirják. Fourth order real space solver for the time-dependent Schrödinger equation with singular Coulomb potential. Computer Physics Communications, 208: 9-28, 2016; doi:10.1016/j.cpc.2016.07.006.

[P2] Szilárd Majorosi, Mihály G. Benedict, and Attila Czirják. Quantum entanglement in strong-field ionization. Physical Review A, 96(4): 043412, 2017;

doi: 10.1103/PhysRevA.96.043412.

[P3] Szilárd Majorosi, Mihály G. Benedict, and Attila Czirják. Improved one-dimensional model potentials for strong-field simulations. Physical Review A, 98(2): 023401, 2018; doi: 10.1103/PhysRevA.98.023401.

\section{Additional refereed research papers and conference proceedings:}

[A1] Attila Czirják, Szilárd Majorosi, Judit Kovács, and Mihály G. Benedict. Emergence of oscillations in quantum entanglement during rescattering. Physica Scripta, 2013(T153): 014013, 2013. doi:10.1088/0031-8949/2013/T153/014013.

[A2] Attila Czirják, Szilárd Majorosi, Judit Kovács, and Mihály G. Benedict. Build-up of quantum entanglement during rescattering. In AIP Conference Proceedings, 1462(1): 88-91. AIP, 2012; doi: 10.1063/1.4736766. 


\section{Bibliography}

[1] M Hentschel, R Kienberger, Ch Spielmann, Georg A Reider, N Milosevic, Thomas Brabec, Paul Corkum, Ulrich Heinzmann, Markus Drescher, and Ferenc Krausz. Attosecond metrology. Nature, 414(6863):509-513, 2001.

[2] PM Paul, ES Toma, P Breger, Genevive Mullot, F Augé, Ph Balcou, HG Muller, and P Agostini. Observation of a train of attosecond pulses from high harmonic generation. Science, 292(5522):1689-1692, 2001.

[3] Reinhard Kienberger, Michael Hentschel, Matthias Uiberacker, Ch Spielmann, Markus Kitzler, Armin Scrinzi, M Wieland, Th Westerwalbesloh, U Kleineberg, Ulrich Heinzmann, et al. Steering attosecond electron wave packets with light. Science, 297(5584): 1144-1148, 2002.

[4] Markus Drescher, Michael Hentschel, R Kienberger, Matthias Uiberacker, Vladislav Yakovlev, Armin Scrinzi, Th Westerwalbesloh, U Kleineberg, Ulrich Heinzmann, and Ferenc Krausz. Time-resolved atomic inner-shell spectroscopy. Nature, 419(6909):803807, 2002.

[5] Andrius Baltuška, Th Udem, M Uiberacker, M Hentschel, E Goulielmakis, Ch Gohle, Ronald Holzwarth, VS Yakovlev, A Scrinzi, TW Hänsch, et al. Attosecond control of electronic processes by intense light fields. Nature, 421(6923):611-615, 2003.

[6] Ferenc Krausz and Misha Ivanov. Attosecond physics. Reviews of Modern Physics, 81 (1):163-234, 2009.

[7] A McPherson, G Gibson, H Jara, U Johann, Ting S Luk, IA McIntyre, Keith Boyer, and Charles K Rhodes. Studies of multiphoton production of vacuum-ultraviolet radiation in the rare gases. Journal of the Optical Society of America B, 4(4):595-601, 1987.

[8] M Ferray, A L'Huillier, XF Li, LA Lompre, G Mainfray, and C Manus. Multipleharmonic conversion of $1064 \mathrm{~nm}$ radiation in rare gases. Journal of Physics B: Atomic, Molecular and Optical Physics, 21(3):L31, 1988.

[9] Gy Farkas and Cs Tóth. Proposal for attosecond light pulse generation using laser induced multiple-harmonic conversion processes in rare gases. Physics Letters A, 168(5): 447-450, 1992. 
[10] SE Harris, JJ Macklin, and TW Hänsch. Atomic scale temporal structure inherent to high-order harmonic generation. Optics communications, 100(5-6):487-490, 1993.

[11] Matthias Uiberacker, Th Uphues, Martin Schultze, Aart Johannes Verhoef, Vladislav Yakovlev, Matthias F Kling, Jens Rauschenberger, Nicolai M Kabachnik, Hartmut Schröder, Matthias Lezius, et al. Attosecond real-time observation of electron tunnelling in atoms. Nature, 446(7136):627-632, 2007.

[12] Adrian L Cavalieri, Norbert Müller, Th Uphues, Vladislav S Yakovlev, Andrius Baltuška, Balint Horvath, B Schmidt, L Blümel, R Holzwarth, S Hendel, et al. Attosecond spectroscopy in condensed matter. Nature, 449(7165):1029-1032, 2007.

[13] Martin Schultze, Markus Fieß, Nicholas Karpowicz, Justin Gagnon, Michael Korbman, Michael Hofstetter, S Neppl, Adrian L Cavalieri, Yannis Komninos, Th Mercouris, et al. Delay in photoemission. Science, 328(5986):1658-1662, 2010.

[14] Stefan Haessler, J Caillat, W Boutu, C Giovanetti-Teixeira, T Ruchon, T Auguste, Z Diveki, P Breger, A Maquet, B Carré, et al. Attosecond imaging of molecular electronic wavepackets. Nature Physics, 6(3):200-206, 2010.

[15] Adrian N Pfeiffer, Claudio Cirelli, Alexandra S Landsman, Mathias Smolarski, Darko Dimitrovski, Lars B Madsen, and Ursula Keller. Probing the longitudinal momentum spread of the electron wave packet at the tunnel exit. Physical Review Letters, 109(8): 083002, 2012.

[16] Dror Shafir, Hadas Soifer, Barry D Bruner, Michal Dagan, Yann Mairesse, Serguei Patchkovskii, Misha Yu Ivanov, Olga Smirnova, and Nirit Dudovich. Resolving the time when an electron exits a tunnelling barrier. Nature, 485(7398):343-346, 2012.

[17] LV Keldysh. Ionization in the field of a strong electromagnetic wave. Soviet Physics JETP, 20(5):1307-1314, 1965.

[18] Sándor Varró and F Ehlotzky. A new integral equation for treating high-intensity multiphoton processes. Il Nuovo Cimento D, 15(11):1371-1396, 1993.

[19] Maciej Lewenstein, Ph Balcou, M Yu Ivanov, Anne L'huillier, and Paul B Corkum. Theory of high-harmonic generation by low-frequency laser fields. Physical Review A, 49 (3):2117, 1994.

[20] M Protopapas, DG Lappas, and PL Knight. Strong field ionization in arbitrary laser polarizations. Physical Review Letters, 79(23):4550, 1997.

[21] Misha Yu Ivanov, Michael Spanner, and Olga Smirnova. Anatomy of strong field ionization. Journal of Modern Optics, 52(2-3):165-184, 2005.

[22] Ariel Gordon, Robin Santra, and Franz X Kärtner. Role of the Coulomb singularity in high-order harmonic generation. Physical Review A, 72(6):063411, 2005. 
[23] MV Frolov, NL Manakov, AM Popov, OV Tikhonova, EA Volkova, AA Silaev, NV Vvedenskii, and Anthony F Starace. Analytic theory of high-order-harmonic generation by an intense few-cycle laser pulse. Physical Review A, 85(3):033416, 2012.

[24] P Eckle, AN Pfeiffer, C Cirelli, A Staudte, R Dörner, HG Muller, M Büttiker, and $\mathrm{U}$ Keller. Attosecond ionization and tunneling delay time measurements in helium. Science, 322(5907):1525-1529, 2008.

[25] Juha Javanainen, Joseph H Eberly, and Qichang Su. Numerical simulations of multiphoton ionization and above-threshold electron spectra. Physical Review A, 38(7):3430, 1988.

[26] Q Su and JH Eberly. Model atom for multiphoton physics. Physical Review A, 44(9): 5997, 1991.

[27] D Bauer. Two-dimensional, two-electron model atom in a laser pulse: Exact treatment, single-active-electron analysis, time-dependent density-functional theory, classical calculations, and nonsequential ionization. Physical Review A, 56(4):3028, 1997.

[28] CC Chirilă, Ingo Dreissigacker, Elmar V van der Zwan, and Manfred Lein. Emission times in high-order harmonic generation. Physical Review A, 81(3):033412, 2010.

[29] AA Silaev, M Yu Ryabikin, and NV Vvedenskii. Strong-field phenomena caused by ultrashort laser pulses: Effective one-and two-dimensional quantum-mechanical descriptions. Physical Review A, 82(3):033416, 2010.

[30] Konstantin Alekseevich Sveshnikov and Dmitrii Igorevich Khomovskii. Schrödinger and Dirac particles in quasi-one-dimensional systems with a Coulomb interaction. Theoretical and Mathematical Physics, 173(2):1587-1603, 2012.

[31] Stefanie Gräfe, Jens Doose, and Joachim Burgdörfer. Quantum phase-space analysis of electronic rescattering dynamics in intense few-cycle laser fields. Journal of Physics B: Atomic, Molecular and Optical Physics, 45(5):055002, 2012.

[32] A Czirják, R Kopold, W Becker, M Kleber, and WP Schleich. The Wigner function for tunneling in a uniform static electric field. Optics communications, 179(1):29-38, 2000.

[33] Sydney Geltman. Bound states in delta function potentials. Journal of Atomic, Molecular, and Optical Physics, 2011, 2011.

[34] C Baumann, H-J Kull, and GM Fraiman. Wigner representation of ionization and scattering in strong laser fields. Physical Review A, 92(6):063420, 2015.

[35] Nicolas Teeny, Enderalp Yakaboylu, Heiko Bauke, and Christoph H Keitel. Ionization time and exit momentum in strong-field tunnel ionization. Physical Review Letters, 116 (6):063003, 2016.

[36] Albert Einstein, Boris Podolsky, and Nathan Rosen. Can quantum-mechanical description of physical reality be considered complete? Physical Review, 47(10):777, 1935. 
[37] MD Reid, PD Drummond, WP Bowen, Eric Gama Cavalcanti, Ping Koy Lam, HA Bachor, Ulrik Lund Andersen, and G Leuchs. Colloquium: the Einstein-Podolsky-Rosen paradox: from concepts to applications. Reviews of Modern Physics, 81(4):1727, 2009.

[38] Samuel L Braunstein and Peter Van Loock. Quantum information with continuous variables. Reviews of Modern Physics, 77(2):513, 2005.

[39] MV Fedorov, MA Efremov, AE Kazakov, KW Chan, CK Law, and JH Eberly. Spontaneous emission of a photon: Wave-packet structures and atom-photon entanglement. Physical Review A, 72(3):032110, 2005.

[40] Jürgen Volz, Markus Weber, Daniel Schlenk, Wenjamin Rosenfeld, Johannes Vrana, Karen Saucke, Christian Kurtsiefer, and Harald Weinfurter. Observation of entanglement of a single photon with a trapped atom. Physical Review Letters, 96(3):030404, 2006.

[41] Shuangyuan Xie, Fei Jia, and Yaping Yang. Dynamic control of the entanglement in the presence of the time-varying field. Optics Communications, 282(13):2642-2649, 2009.

[42] Sándor Varró. Entangled photon-electron states and the number-phase minimum uncertainty states of the photon field. New Journal of Physics, 10(5):053028, 2008.

[43] Sándor Varró. Entangled states and entropy remnants of a photon-electron system. Physica Scripta, 2010(T140):014038, 2010.

[44] Assaf Tal and Gershon Kurizki. Translational entanglement via collisions: How much quantum information is obtainable? Physical Review Letters, 94(16):160503, 2005.

[45] Mihály G Benedict, Judit Kovács, and Attila Czirják. Time dependence of quantum entanglement in the collision of two particles. Journal of Physics A: Mathematical and Theoretical, 45(8):085304, 2012.

[46] I Nagy, I Aldazabal, and A Rubio. Exact time evolution of the pair distribution function for an entangled two-electron initial state. Physical Review A, 86(2):022512, 2012.

[47] HJ Kull. Position-momentum correlations in electron-ion scattering in strong laser fields. New Journal of Physics, 14(5):055013, 2012.

[48] Ebrahim Ghanbari-Adivi and Morteza Soltani. Entanglement generation between two colliding particles. The European Physical Journal D, 68(11):336, 2014.

[49] R Feder, F Giebels, and H Gollisch. Entanglement creation in electron-electron collisions at solid surfaces. Physical Review B, 92(7):075420, 2015.

[50] MV Fedorov, MA Efremov, AE Kazakov, KW Chan, CK Law, and JH Eberly. Packet narrowing and quantum entanglement in photoionization and photodissociation. Physical Review A, 69(5):052117, 2004. 
[51] MV Fedorov, MA Efremov, PA Volkov, and JH Eberly. Short-pulse or strong-field breakup processes: a route to study entangled wave packets. Journal of Physics B: Atomic, Molecular and Optical Physics, 39(13):S467, 2006.

[52] MV Ammosov, NB Delone, and VP Krainov. Tunnel ionization of complex atoms and of atomic ions in an alternating electric field. Soviet Physics JETP, 64:1191, 1986.

[53] Paul B Corkum. Plasma perspective on strong field multiphoton ionization. Physical Review Letters, 71(13):1994, 1993.

[54] Giuseppe Sansone, E Benedetti, Francesca Calegari, Caterina Vozzi, Lorenzo Avaldi, Roberto Flammini, Luca Poletto, P Villoresi, C Altucci, R Velotta, et al. Isolated singlecycle attosecond pulses. Science, 314(5798):443-446, 2006.

[55] Juan J Carrera, Xiao-Min Tong, and Shih-I Chu. Creation and control of a single coherent attosecond XUV pulse by few-cycle intense laser pulses. Physical review A, 74(2): 023404, 2006.

[56] Nicolas Camus, Enderalp Yakaboylu, Lutz Fechner, Michael Klaiber, Martin Laux, Yonghao Mi, Karen Z Hatsagortsyan, Thomas Pfeifer, Christoph H Keitel, and Robert Moshammer. Experimental evidence for quantum tunneling time. Physical Review Letters, 119(2):023201, 2017.

[57] Brian Harold Bransden and Charles Jean Joachain. Physics of Atoms and Molecules. Pearson Education India, 2003.

[58] Thomas Brabec and Henry Kapteyn. Strong field laser physics, volume 1. Springer, 2008.

[59] David Jeffery Griffiths. Introduction to Quantum Mechanics. Pearson Education India, 2005.

[60] Guillermo Palma and Ulrich Raff. The one-dimensional hydrogen atom revisited. Canadian Journal of Physics, 84(9):787-800, 2006.

[61] Rodney Loudon. One-dimensional hydrogen atom. American Journal of Physics, 27(9): 649-655, 1959.

[62] AD Bandrauk, S Chelkowski, Dennis J Diestler, J Manz, and K-J Yuan. Quantum simulation of high-order harmonic spectra of the hydrogen atom. Physical Review A, 79(2): 023403, 2009.

[63] Charles Jean Joachain, Niels J Kylstra, and Robert M Potvliege. Atoms in intense laser fields. Cambridge University Press, 2012.

[64] Nicolas Teeny, Christoph H Keitel, and Andreas Mielke. Tunneling Time, Exit Time and Exit Momentum in Strong Field Tunnel Ionization. PhD thesis, Ruprecht-KarlsUniversität Heidelberg, 2016. 
[65] IV Puzynin, AV Selin, and SI Vinitsky. Magnus-factorized method for numerical solving the time-dependent Schrödinger equation. Computer Physics Communications, 126(1): 158-161, 2000.

[66] Sergio Blanes, Fernando Casas, JA Oteo, and José Ros. The Magnus expansion and some of its applications. Physics Reports, 470(5):151-238, 2009.

[67] IV Puzynin, AV Selin, and SI Vinitsky. A high-order accuracy method for numerical solving of the time-dependent Schrödinger equation. Computer Physics Communications, 123(1):1-6, 1999.

[68] W van Dijk and FM Toyama. Accurate numerical solutions of the time-dependent Schrödinger equation. Physical Review E, 75(3):36707, 2007.

[69] HG Muller. An efficient propagation scheme for the time-dependent Schrödinger equation in the velocity gauge. Laser Physics, 9:138-148, 1999.

[70] Dieter Bauer and Peter Koval. Qprop: A Schrödinger-solver for intense laser-atom interaction. Computer Physics Communications, 174(5):396-421, 2006.

[71] Edward S Smyth, Jonathan S Parker, and Ken T Taylor. Numerical integration of the time-dependent Schrödinger equation for laser-driven helium. Computer Physics Communications, 114(1):1-14, 1998.

[72] Luca Argenti, Renate Pazourek, Johannes Feist, Stefan Nagele, Matthias Liertzer, Emil Persson, Joachim Burgdörfer, and Eva Lindroth. Photoionization of helium by attosecond pulses: Extraction of spectra from correlated wave functions. Physical Review A, 87(5): 053405, 2013.

[73] Barry I Schneider, Lee A Collins, and SX Hu. Parallel solver for the time-dependent linear and nonlinear Schrödinger equation. Physical Review E, 73(3):036708, 2006.

[74] Ariel Gordon, Christian Jirauschek, and Franz X Kärtner. Numerical solver of the timedependent Schrödinger equation with Coulomb singularities. Physical Review A, 73(4): 042505, 2006.

[75] IK Gainullin and MA Sonkin. High-performance parallel solver for 3D time-dependent Schrödinger equation for large-scale nanosystems. Computer Physics Communications, 188:68-75, 2015.

[76] Claude Leforestier, RH Bisseling, Charly Cerjan, MD Feit, Rich Friesner, A Guldberg, A Hammerich, G Jolicard, W Karrlein, H-D Meyer, et al. A comparison of different propagation schemes for the time dependent Schrödinger equation. Journal of Computational Physics, 94(1):59-80, 1991.

[77] Toshiaki Iitaka. Solving the time-dependent Schrödinger equation numerically. Physical Review E, 49(5):4684, 1994. 
[78] Alberto Castro, Miguel AL Marques, and Angel Rubio. Propagators for the timedependent Kohn-Sham equations. The Journal of chemical physics, 121(8):3425-3433, 2004.

[79] William H. Press, Saul A. Teukolsky, William T. Vetterling, and Brian P. Flannery. Numerical Recipes 3rd Edition: The Art of Scientific Computing. Cambridge University Press, 3 edition, 2007.

[80] James M Varah. On the solution of block-tridiagonal systems arising from certain finitedifference equations. Mathematics of Computation, 26(120):859-868, 1972.

[81] RM Wilcox. Exponential operators and parameter differentiation in quantum physics. Journal of Mathematical Physics, 8(4):962-982, 1967.

[82] Robert I McLachlan and G Reinout W Quispel. Splitting methods. Acta Numerica, 11: 341-434, 2002.

[83] Yesim Yazici. Operator splitting methods for differential equations. PhD thesis, Izmir Institute of Technology, 2010.

[84] Gilbert Strang. On the construction and comparison of difference schemes. SIAM Journal on Numerical Analysis, 5(3):506-517, 1968.

[85] Siu A Chin. Symplectic integrators from composite operator factorizations. Physics Letters A, 226(6):344-348, 1997.

[86] Masuo Suzuki. Hybrid exponential product formulas for unbounded operators with possible applications to Monte Carlo simulations. Physics Letters A, 201(5):425-428, 1995.

[87] André D Bandrauk and Hai Shen. Higher order exponential split operator method for solving time-dependent Schrödinger equations. Canadian Journal of Chemistry, 70(2): 555-559, 1992.

[88] André D Bandrauk and Hai Shen. Exponential split operator methods for solving coupled time-dependent Schrödinger equations. The Journal of chemical physics, 99(2):1185$1193,1993$.

[89] Andre D Bandrauk and Huizhong Lu. Exponential propagators (integrators) for the timedependent Schrödinger equation. Journal of Theoretical and Computational Chemistry, 12(06):1340001, 2013.

[90] Siu A Chin, S Janecek, and E Krotscheck. Any order imaginary time propagation method for solving the Schrödinger equation. Chemical Physics Letters, 470(4):342-346, 2009.

[91] Lauri Lehtovaara, Jari Toivanen, and Jussi Eloranta. Solution of time-independent Schrödinger equation by the imaginary time propagation method. Journal of Computational Physics, 221(1):148-157, 2007. 
[92] S Blanes and PC Moan. Splitting methods for the time-dependent Schrödinger equation. Physics Letters A, 265(1):35-42, 2000.

[93] Siu A Chin and Chia-Rong Chen. Fourth order gradient symplectic integrator methods for solving the time-dependent Schrödinger equation. The Journal of Chemical Physics, 114(17):7338-7341, 2001.

[94] Hanquan Wang. Numerical studies on the split-step finite difference method for nonlinear Schrödinger equations. Applied Mathematics and Computation, 170(1):17-35, 2005.

[95] Jing Shen, EI Wei, Zhixiang Huang, Mingsheng Chen, and Xianliang Wu. High-order symplectic FDTD scheme for solving a time-dependent Schrödinger equation. Computer Physics Communications, 184(3):480-492, 2013.

[96] JH Eberly. Schmidt analysis of pure-state entanglement. Laser Physics, 16(6):921-926, 2006.

[97] Shunlong Luo and Zhengmin Zhang. Entanglement and interference. Physics Letters A, 315(3):189-193, 2003.

[98] Christopher Eltschka and Jens Siewert. Quantifying entanglement resources. Journal of Physics A: Mathematical and Theoretical, 47(42):424005, 2014.

[99] Chien-Hao Lin and Yew Kam Ho. Calculation of von Neumann entropy for hydrogen and positronium negative ions. Physics Letters A, 378(38):2861-2865, 2014.

[100] Benjamin Schumacher. Quantum coding. Physical Review A, 51(4):2738, 1995.

[101] HA Carteret, A Higuchi, and A Sudbery. Multipartite generalization of the Schmidt decomposition. Journal of Mathematical Physics, 41(12):7932-7939, 2000.

[102] Alfred Wehrl. General properties of entropy. Reviews of Modern Physics, 50(2):221, 1978.

[103] Claude E Shannon and Warren Weaver. The Mathematical Theory of Communication. University of Illinois Press, 1949.

[104] Nicolas J Cerf and Chris Adami. Negative entropy and information in quantum mechanics. Physical Review Letters, 79(26):5194, 1997.

[105] Nicolas J Cerf and Christoph Adami. Quantum extension of conditional probability. Physical Review A, 60(2):893, 1999.

[106] C. Cohen-Tannoudji, B. Diu, and F. Laloë. Quantum Mechanics. Quantum Mechanics. Wiley, 1977.

[107] AA Karawia. A computational algorithm for solving periodic penta-diagonal linear systems. Applied Mathematics and Computation, 174(1):613-618, 2006. 
[108] Xufei Sun, Min Li, Jizhou Yu, Yongkai Deng, Qihuang Gong, and Yunquan Liu. Calibration of the initial longitudinal momentum spread of tunneling ionization. Physical Review A, 89(4):045402, 2014.

[109] L Guo, SS Han, and J Chen. Time-energy analysis of above-threshold ionization in few-cycle laser pulses. Physical Review A, 86(5):053409, 2012.

[110] C Zagoya, J Wu, M Ronto, DV Shalashilin, and C Figueira de Morisson Faria. Quantum and semiclassical phase-space dynamics of a wave packet in strong fields using initialvalue representations. New Journal of Physics, 16(10):103040, 2014.

[111] C Baumann, H-J Kull, and GM Fraiman. Wigner representation of ionization and scattering in strong laser fields. Physical Review A, 92(6):063420, 2015.

[112] Peter A Bouvrie, Ana P Majtey, Malte C Tichy, Jesus S Dehesa, and Angel R Plastino. Entanglement and the Born-Oppenheimer approximation in an exactly solvable quantum many-body system. The European Physical Journal D, 68(11):346, 2014.

[113] NH March, A Cabo, F Claro, and GGN Angilella. Proposed definitions of the correlation energy density from a Hartree-Fock starting point: The two-electron Moshinsky model atom as an exactly solvable model. Physical Review A, 77(4):042504, 2008.

[114] Arun K Pati. Existence of the Schmidt decomposition for tripartite systems. Physics Letters A, 278(3):118-122, 2000.

[115] Michael H Beck, Andreas Jäckle, GA Worth, and H-D Meyer. The multiconfiguration time-dependent Hartree (MCTDH) method: a highly efficient algorithm for propagating wavepackets. Physics Reports, 324(1):1-105, 2000.

[116] Walter Kohn. Nobel lecture: Electronic structure of matter - wave functions and density functionals. Reviews of Modern Physics, 71(5):1253, 1999.

[117] Carsten A Ullrich. Time-dependent density-functional theory: concepts and applications. Oxford University Press, 2011.

[118] Walter Kohn and Lu Jeu Sham. Self-consistent equations including exchange and correlation effects. Physical Review, 140(4A):A1133, 1965.

[119] Curt A Moyer. Numerov extension of transparent boundary conditions for the Schrödinger equation in one dimension. American Journal of Physics, 72(3):351-358, 2004.

[120] Kôdi Husimi. Miscellanea in elementary quantum mechanics, ii. Progress of Theoretical Physics, 9(4):381-402, 1953. 


\section{APPEndix A}

\section{High-order finite difference formulas}

Let us denote our general function for the derivation of the finite difference formulas [79] by $y(x)$ and the uniformly discretized coordinate by $x_{k}=k \cdot \Delta x, k \in\left[0, N_{x}\right], \Delta x=x_{\max } / N_{x}$. We need these formulas for the expressions of the spatial derivatives within the Crank-Nicolson schemes.

The usual starting point of the procedure is the Taylor expansion of $y(x)$ around $x_{k}$ as

$$
y_{k+n}=y_{k}+n \cdot y_{k}^{(1)} \Delta x+\frac{n^{2}}{2 !} \cdot y_{k}^{(2)} \Delta x^{2}+\frac{n^{3}}{3 !} \cdot y_{k}^{(3)} \Delta x^{3}+\frac{n^{4}}{4 !} \cdot y_{k}^{(4)} \Delta x^{4}+\frac{n^{5}}{5 !} \cdot y_{k}^{(5)} \Delta x^{5}+O\left(\Delta x^{6}\right),
$$

where $y_{k}^{(n)}$ denotes the $n$th derivative of $y(x)$ evaluated at point $x_{k}$. Using only points $y_{k-1}, y_{k}, y_{k+1}$ we will arrive at the well-known three-point formulas of the first and the second derivatives, both with second-order accuracy:

$$
y_{k}^{(1)}=\frac{-y_{k-1}+y_{k+1}}{2 \Delta x}+O\left(\Delta x^{2}\right), y_{k}^{(2)}=\frac{y_{k-1}-2 y_{k}+y_{k+1}}{\Delta x^{2}}+O\left(\Delta x^{2}\right) .
$$

Using (A.1) at multiple gridpoints $x_{k+n}$ with fixed $k$, and eliminating the unnecessary terms according to desired order of $\Delta x$, we can get a generalized procedure to acquire high-order finite difference formulas [68]. For example, using the five points $y_{k-2}, y_{k-1}, y_{k}, y_{k+1}, y_{k+2}$ we can get the fourth-order approximations,

$$
\begin{gathered}
y_{k}^{(1)}=\frac{y_{k-2}-8 y_{k-1}+8 y_{k+1}-y_{k+2}}{12 \Delta x}+O\left(\Delta x^{4}\right), \\
y_{k}^{(2)}=\frac{-y_{k-2}+16 y_{k-1}-30 y_{k}+16 y_{k+1}-y_{k+2}}{12 \Delta x^{2}}+O\left(\Delta x^{4}\right) .
\end{gathered}
$$

The seven-point symmetric formulas can be acquired the same way using $y_{k-3}, y_{k-2}, y_{k-1}, y_{k}$, $y_{k+1}, y_{k+2}, y_{k+3}$ :

$$
\begin{gathered}
y_{k}^{(1)}=\frac{-3 y_{k-3}+27 y_{k-2}-135 y_{k-1}+135 y_{k+1}-27 y_{k+2}+3 y_{k+2}}{180 \Delta x}+O\left(\Delta x^{6}\right), \\
y_{k}^{(2)}=\frac{2 y_{k-3}-27 y_{k-2}+270 y_{k-1}-490 y_{k}+270 y_{k+1}-27 y_{k+2}+2 y_{k+2}}{180 \Delta x}+O\left(\Delta x^{6}\right) .
\end{gathered}
$$

In general, the $2 n$-order finite difference formulas of the derivatives are expressed as the follow- 
ing linear combinations [68]:

$$
y_{k}^{(1)}=\sum_{s=-n}^{n} \frac{\operatorname{sgn}(\mathrm{s}) b_{|s|}^{(n)}}{\Delta z} y_{k+s}+O\left(\Delta x^{2 n}\right), \quad \text { and } \quad y_{k}^{(2)}=\sum_{s=-n}^{n} \frac{c_{|s|}^{(n)}}{\Delta z^{2}} y_{k+s}+O\left(\Delta x^{2 n}\right) .
$$

However, the higher-order the difference formula is, the more analytic conditions $y(x)$ should satisfy.

For the Neumann boundary conditions, it is sometimes a necessity to use one-sided (forward or backward) finite differences especially if the first derivative of the function is discontinuous. The simplest of this is the first-order accurate forward difference formula, which we can get directly using $y_{k}, y_{k+1}$ points as $y_{k}^{(1)}=\left(y_{k+1}-y_{k}\right) / \Delta x+O(\Delta x)$. However, higher-order formulas of this type can be derived straightforwardly using multiple points of Taylor-expansions on the same side of $x_{k}$. For the 3-, 5- and 7-point finite difference formulas we get the following results:

$$
\begin{gathered}
y_{k}^{(1)}= \pm \frac{-3 y_{k}+4 y_{k \pm 1}-y_{k \pm 2}}{2 \Delta x}+O\left(\Delta x^{2}\right), \\
y_{k}^{(1)}= \pm \frac{-25 y_{k}+48 y_{k \pm 1}-36 y_{k \pm 2}+16 y_{k \pm 3}-3 y_{k \pm 4}}{12 \Delta x}+O\left(\Delta x^{4}\right), \\
y_{k}^{(1)}= \pm \frac{-147 y_{k}+360 y_{k \pm 1}-450 y_{k \pm 2}+400 y_{k \pm 3}-225 y_{k \pm 4}-72 y_{k \pm 5}-10 y_{k \pm 6}}{60 \Delta x}+O\left(\Delta x^{6}\right) .
\end{gathered}
$$

The negative signs apply for the backward difference formulas. 


\section{APPENDiX B}

\section{The Numerov z-line propagator algorithm}

In this appendix, we construct an efficient way of evaluating $e^{-i \Delta t H_{z}}$ line-by-line accompanying the hybrid splitting algorithm of Section 4.2 using cylindrical coordinates $z, \rho$. This is based on the second-order Crank-Nicolson algorithm with Numerov-extension [119], which also provides at least fourth-order accuracy in $\Delta z$, and it reduces the numerical costs because it uses three-point finite differences and tridiagonal equations instead of five-point differences and pentadiagonal equations. We will also outline an optimization technique for 1D CrankNicolson schemes which is makes the computations even more efficient when they are applied to $2 \mathrm{D}$ problems.

Let us fix the value of coordinate $\rho$ (i.e we choose $j=$ const) and we denote $\Psi_{i}\left(t_{k}\right)=$ $\Psi_{i, j}\left(t_{k}\right)$. Here we consider the Hamiltonian in the form of $H_{z}=\beta \partial_{z}^{2}+V(z, t)$ along this line since the procedure below allows to include a potential of this form, which is however not present in our hybrid splitting scheme (4.21). Again, we start with the second-order approximation of the exponential operator $e^{-i \Delta t H_{z}}$ as in (2.10)

$$
\left(1+\alpha \beta \partial_{z}^{2}+\alpha V\right) \Psi\left(t_{k+1}\right)=\left(1-\alpha \beta \partial_{z}^{2}-\alpha V\right) \Psi\left(t_{k}\right)
$$

with $\alpha=i \Delta t / 2, \beta=-1 / 2 \mu, V=V\left(z, t_{k+1 / 2}\right)$. We will use a discretized Laplacian $\widetilde{L}_{z}$ based on the standard three-point finite difference method (A.2), however, we will also need the leading term of the error:

$$
\widetilde{L}_{z} \Psi_{i}=\left.\frac{\Psi_{i-1}-2 \Psi_{i}+\Psi_{i+1}}{\Delta z^{2}} \approx \frac{\partial^{2} \Psi}{\partial z^{2}}\right|_{z_{i}}-\left.\frac{\Delta z^{2}}{12} \frac{\partial^{4} \Psi}{\partial z^{4}}\right|_{z_{i}}+O\left(\Delta z^{4}\right) .
$$

To proceed, we introduce the auxiliary variable $Y\left(t_{k}\right)=\Psi\left(t_{k+1}\right)+\Psi\left(t_{k}\right)$ and rewrite the equation (B.1) as

$$
\left(\alpha \beta \partial_{z}^{2}\right) Y\left(t_{k}\right)=2 \Psi\left(t_{k}\right)-(1+\alpha V) Y\left(t_{k}\right)
$$

Then, we discretize the equation (B.3) using (B.2):

$$
\left(\alpha \beta \widetilde{L}_{z}\right) Y_{i}\left(t_{k}\right)=2 \Psi_{i}\left(t_{k}\right)-\left(1+\alpha V_{i}\right) Y_{i}\left(t_{k}\right)+\left.\alpha \beta \frac{\Delta z^{2}}{12} \frac{\partial^{4} Y}{\partial z^{4}}\right|_{i}
$$


Now, we evaluate the error term with $\partial_{z}^{4} Y_{i}\left(t_{k}\right)=\widetilde{L}_{z}\left(\partial_{z}^{2} Y_{i}\left(t_{k}\right)\right)+O\left(\Delta z^{2}\right)$ also using the right hand side of (B.3) to get to the result of the form

$$
\left(1+\alpha V_{i}+\alpha \beta \widetilde{L}_{z}+\frac{\Delta z^{2}}{12} \cdot \widetilde{L}_{z}\left(1+\alpha V_{i}\right)\right) Y_{i}\left(t_{k}\right)=2 \Psi_{i}\left(t_{k}\right)+\frac{\Delta z^{2}}{6} \widetilde{L}_{z} \Psi_{i}\left(t_{k}\right)
$$

Restoring the equations for $\Psi_{i}\left(t_{k+1}\right)$ we arrive at the Numerov-extended Crank-Nicolson algorithm as

$$
\left(1+\alpha V_{i}+\alpha \beta \widetilde{L}_{z}+\frac{\Delta z^{2}}{12} \widetilde{L}_{z}\left(1+\alpha V_{i}\right)\right) \Psi_{i}\left(t_{k+1}\right)=\left(1-\alpha V_{i}-\alpha \beta \widetilde{L}_{z}+\frac{\Delta z^{2}}{12} \widetilde{L}_{z}\left(1-\alpha V_{i}\right)\right) \Psi_{i}\left(t_{k}\right)
$$

It is interesting to note that by reverse engineering from the Padé-form, we get a discrete Hamiltonian of the form

$$
\widetilde{H}_{z, i}=V_{i}+\beta \widetilde{L}_{z}-i \frac{\Delta z^{2}}{6 \Delta t} \widetilde{L}_{z}+\frac{\Delta z^{2}}{12} \widetilde{L}_{z} V_{i}
$$

The extra terms in (B.7) improve the solution to the fifth order in $\Delta z$, however, one should note that (i) $\widetilde{L}_{z}\left(V_{i} \Psi_{i}\left(t_{k}\right)\right)$ is part of $\widetilde{H}_{z} \Psi\left(t_{k}\right)$, requiring the potential to be continuously differentiable, (ii) $\widetilde{H}_{z}$ is no longer strictly self-adjoint.

In (B.6) we have arrived at a tridiagonal system of linear equations of the form

$$
\left[\begin{array}{cccccc}
b_{0} & c_{0} & 0 & 0 & \cdots & 0 \\
a_{1} & b_{1} & c_{1} & 0 & \ldots & 0 \\
0 & a_{2} & b_{2} & c_{2} & \ldots & 0 \\
\vdots & \vdots & \ddots & \ddots & \ddots & \vdots \\
0 & 0 & \cdots & a_{N_{z}-1} & b_{N_{z}-1} & c_{N_{z}-1} \\
0 & 0 & \cdots & 0 & a_{N_{z}} & b_{N_{z}}
\end{array}\right]\left[\begin{array}{c}
\Psi_{0}\left(t_{k+1}\right) \\
\Psi_{1}\left(t_{k+1}\right) \\
\Psi_{2}\left(t_{k+1}\right) \\
\vdots \\
\Psi_{N_{z}-1}\left(t_{k+1}\right) \\
\Psi_{N_{z}}\left(t_{k+1}\right)
\end{array}\right]=\left[\begin{array}{c}
y_{0} \\
y_{1} \\
y_{2} \\
\vdots \\
y_{N_{z}-1} \\
y_{N_{z}}
\end{array}\right]
$$

which after forward Gaussian-elimination reads

$$
\left[\begin{array}{cccccc}
\tilde{b}_{0} & c_{0} & 0 & 0 & \cdots & 0 \\
0 & \tilde{b}_{1} & c_{1} & 0 & \cdots & 0 \\
0 & 0 & \tilde{b}_{2} & c_{2} & \cdots & 0 \\
\vdots & \vdots & \ddots & \ddots & \ddots & \vdots \\
0 & 0 & \cdots & 0 & \tilde{b}_{N_{z}-1} & c_{N_{z}-1} \\
0 & 0 & \cdots & 0 & 0 & \tilde{b}_{N_{z}}
\end{array}\right]\left[\begin{array}{c}
\Psi_{0}\left(t_{k+1}\right) \\
\Psi_{1}\left(t_{k+1}\right) \\
\Psi_{2}\left(t_{k+1}\right) \\
\vdots \\
\Psi_{N_{z}-1}\left(t_{k+1}\right) \\
\Psi_{N_{z}}\left(t_{k+1}\right)
\end{array}\right]=\left[\begin{array}{c}
\tilde{y}_{0} \\
\tilde{y}_{1} \\
\tilde{y}_{2} \\
\vdots \\
\tilde{y}_{N_{z}-1} \\
\tilde{y}_{N_{z}}
\end{array}\right]
$$

In the coefficient matrix of (B.8) and (B.9) we denoted

$$
\begin{gathered}
a_{i}=\left\{1+\alpha V_{i-1}+12 \alpha \beta / \Delta z^{2} \quad \text { if } i=1, \ldots, N_{z},\right. \\
c_{i}=\left\{1+\alpha V_{i+1}+12 \alpha \beta / \Delta z^{2} \quad \text { if } i=0, \ldots, N_{z}-1,\right. \\
b_{i}=\left\{10+10 \alpha V_{i}-24 \alpha \beta / \Delta z^{2} \quad \text { if } i=0, \ldots, N_{z},\right.
\end{gathered}
$$




$$
\tilde{b}_{i}= \begin{cases}b_{i} & \text { if } i=0, \\ b_{i}-\left(a_{i} / \tilde{b}_{i-1}\right) c_{i-1} & \text { if } i=1, \ldots, N_{z} .\end{cases}
$$

The right hand side and the solution of (B.9) are given by the following expressions:

$$
\begin{gathered}
\tilde{y}_{i}= \begin{cases}\left(20-b_{i}\right) \Psi_{0}+\left(2-c_{i}\right) \Psi_{1} & \text { if } i=0, \\
\left(2-a_{i}\right) \Psi_{i-1}+\left(20-b_{i}\right) \Psi_{i}+\left(2-c_{i}\right) \Psi_{i+1}-\left(a_{i} / \tilde{b}_{i-1}\right) \tilde{y}_{i-1} & \text { if } i=1, \ldots, N_{z}-1, \\
\left(2-a_{i}\right) \Psi_{N_{z}-1}+\left(20-b_{i}\right) \Psi_{N_{z}}-\left(a_{i} / \tilde{b}_{i-1}\right) \tilde{y}_{N_{z}-1} & \text { if } i=N_{z},\end{cases} \\
\Psi_{i}\left(t_{k+1}\right)= \begin{cases}\tilde{y}_{N_{z}} / \tilde{b}_{N_{z}} & \text { if } i=N_{z}, \\
\left(\tilde{y}_{i}-c_{i} \Psi_{i+1}\left(t_{k+1}\right)\right) / \tilde{b}_{i} & \text { if } i=N_{z}-1, \ldots, 0 .\end{cases}
\end{gathered}
$$

This completes the 1D propagation method.

Now let us return to the $2 \mathrm{D}$ problem. Because the discrete Hamiltonian $H_{z}$ is independent from the value of $\rho$, the corresponding coefficient matrix of (B.9) is the same for each $j$-line. This means that we need to do the forward elimination (B.13) only once, then it is sufficient to perform only the forward (B.14) and the backward substitution (B.15) steps for each $j$-line in order to acquire the solution. This yields a factor of 2 speedup in the evaluation of $e^{-i \Delta t H_{z}}$ with three-point finite differences, if we have many lines to propagate and cache the appropriate variables. This latter can also be viewed as an LU decomposition based optimization [79]. 


\section{APPENDix C}

\section{The forced harmonic oscillator}

The TDSE for the axially symmetric (i.e. $m=0$ ) 3D forced harmonic oscillator (FHO) in cylindrical coordinates is the following:

$$
i \frac{\partial}{\partial t} \Psi(z, \rho, t)=\left[\beta \frac{\partial^{2}}{\partial z^{2}}+\beta \frac{\partial^{2}}{\partial \rho^{2}}+\frac{\beta}{\rho} \frac{\partial}{\partial \rho}+\frac{1}{2} \mu \omega_{0}^{2}\left(z^{2}+\rho^{2}\right)+f(t) z\right] \Psi(z, \rho, t)
$$

where $\beta=-1 / 2 \mu$. Using the separability in $z$ and $\rho$ coordinates, we can reduce this problem to a one-dimensional time-dependent one, which was solved by K. Husimi [120]. Then, the analytical time-dependent wave function is of the form

$$
\Psi^{\mathrm{A}}(z, \rho, t)=\chi(z-\xi(t), \rho, t) \exp \left(i \mu(z-\xi(t)) \dot{\xi}(t)+i \int_{0}^{t} \mathscr{L}\left(t^{\prime}\right) \mathrm{d} t^{\prime}\right)
$$

where $\chi(z, \rho, t)$ is a solution of the 3D field-free quantum harmonic oscillator problem with axial symmetry. We define it to be the ground state of the field-free problem $(\mathrm{H}-000)$ :

$$
\chi_{000}(z, \rho, t)=\left[\frac{\mu \omega_{0}}{\pi}\right]^{3 / 4} e^{-\frac{1}{2} \mu \omega_{0}\left(z^{2}+\rho^{2}\right)} e^{-i \frac{3}{2} \omega_{0} t} .
$$

In formula (C.2) the symbol $\mathscr{L}(t)$ denotes the Lagrangian of the corresponding classical system:

$$
\mathscr{L}(t)=\frac{1}{2} \mu \dot{\xi}(t)^{2}-\frac{1}{2} \mu \omega_{0}^{2} \xi(t)^{2}-f(t) \xi(t),
$$

and $\xi(t)$ is the solution of the initial value problem

$$
\ddot{\xi}(t)=f(t) / \mu-\omega_{0}^{2} \xi(t) \text {, with }\{\xi(0)=0, \dot{\xi}(0)=0\} .
$$

We set $f(t)=F \sin \omega_{\mathrm{F}} t$, then the previous equation is that of the forced harmonic oscillator has the solution:

$$
\xi(t)=\frac{F / \mu}{\omega_{\mathrm{F}}^{2}-\omega_{0}^{2}}\left[\sin \omega_{\mathrm{F}} t-\frac{\omega_{\mathrm{F}}}{\omega_{0}} \sin \omega_{0} t\right] .
$$

These formulas define the analytical solution that we use in Section 4.3.2 as one of our test cases. There, in Fig. 4.3, we also plot $\xi(t)$, which is actually the expectation value of the coordinate $z$, and the different terms of the phase in (C.2). 